\title{
The Staphylinidae of Armenia and Nagorno-Karabakh (Coleoptera)
}

With 65 figures, 8 maps and 4 tables

VOLKER ASSING ${ }^{1}$ and MICHAEL SCHÜLKE ${ }^{2}$

1 Gabelsbergerstraße 2, 30163 Hannover, Germany. - vassing.hann@t-online.de
2 Museum für Naturkunde, Invalidenstraße 43,10115 Berlin, Germany. - mschuelke.berlin@t-online.de
Published on 2019-06-24
DOI:10.21248/contrib.entomol.69.1.091-173

\begin{abstract}
The previously largely neglected and poorly known staphylinid faunas of Armenia and Nagorno-Karabakh are addressed. Based on a study of more than 31,000 Staphylinidae recently collected in various habitats and using different methods, and on a critical evaluation of previous literature records, a checklist of the faunas of Armenia and Nagorno-Karabakh is compiled. The fauna of Armenia currently includes 675, that of Nagorno-Karabakh 198 named species. Nevertheless, it is concluded that the species inventory of both regions, especially that of Nagorno-Karabakh, is still far from complete. As many as 262 and 183 species are reported from Armenia and Nagorno-Karabakh, respectively, for the first time. A list of 99 species erroneously or doubtfully recorded from the study region is provided. A comparison with the species number and systematic composition of the faunas of other Caucasian countries and regions revealed that (a) their known diversities are significantly lower than should be expected and (b) a remarkably high proportion (nearly $40 \%$ ) of Aleocharinae in the faunas of Armenia and Nagorno-Karabakh, with the genus Atheta Thomson, 1858 alone accounting for approximately $10 \%$ of the total diversity in Armenia. The faunas of Armenia and Nagorno-Karabakh are primarily composed of widespread species, many of them Caspian (Caucasian) and Iranian elements, and remarkably few endemics. Only 23 species, thirteen of the Aleocharinae (most of them belonging to the genus Geostiba Thomson, 1858), four of the Pselaphinae, five of Scydmaeninae, and one of Staphylininae are classified as regional endemics, and one species of Aleocharinae from the peak region of Mount Khustup is classified as a local endemic. The distribution of one addititional species is confined to South Armenia and adjacent parts of North Iran. A number of species is currently known only from Armenia, but of doubtful taxonomic status or unlikely to represent endemics. Records of some species in Armenia and/or Nagorno-Karabakh revealed some remarkably discontinuous distributions with gaps of up to approximately $2,800 \mathrm{~km}$; three of these distributions are mapped. Fourteen species are newly described: Omalium kociani Zanetri spec. nov. (Armenia: Jermuk) of the Omaliinae, Proteinus baculatus Assing spec. nov. (Armenia; Northeast Turkey) of the Proteininae, Bryaxis armeniacus Brachat spec. nov. (Armenia: NW Hrazdan) and B. meghruicus Brachat spec. nov. (South Armenia: Meghru range) of the Pselaphinae, Atheta (Paralpinia) meghruica Assing spec. nov. (South Armenia: Meghru range), Bellatheta khustupica Assing spec. nov. (South Armenia: Mount Khustup), Calodera alticola Assing spec. nov. (Armenia: Mount Karkar), and Tachyusa unguis Assing spec. nov. (South Armenia) of the Aleocharinae, Anotylus hamatoides SchüLKe spec. nov. (Armenia) of the Oxytelinae, Euconnus (Tetramelus) longilaminatus Meyвонм spec. nov. (North Armenia), E. (T.) tavushus Меувонм spec. nov. (North Armenia), E. (T.) karabakhus Mеувонм spec. nov. (Nagorno-Karabakh), Neuraphes (Paraphes) gomarantsus Meyвонм spec. nov. (South Armenia: Meghru range), and N. (P.) syunikus Меувонм spec. nov. (South Armenia) of the Scydmaeninae. Eight synonymies and one revalidation are established: Dialycera minuta Luze, 1906 = Phyllodrepa armena IABlokoff-Khnzorian, 1959, syn. nov.; Mycetoporus silvaticus IAblokoff-Khnzorian, 1962 = M. dispersus Schülke \& Kocian, 2000, syn. nov.;
\end{abstract}


Aleochara subtumida (Hосннuтн, 1859) = Aleochara khnzoriani AmiRYAN, 1999, syn. nov.; Platystethus cephalotes Eppelsheim, 1878, revalidated (previously synonym of P. laevis Märkel \& Kiesenwetter, 1848) = P. oblongopunctatus Roubal, 1911, syn. nov.; Euconnus lalvarensis IAblokoff-Khnzorian, 1964 = Euconnus pseudorobustus Franz, 1986, syn. nov.; Astenus rufopacus Reitter, 1909 = A. baali Coiffait, 1960, syn. nov.; Heterothops dissimilis (Gravenhorst, 1802) = H. armeniacus CoIfFait, 1977, syn. nov.; Heterothops praevius Erichson, $1839=$ Heterothops montanus IABLOKOFF-KhNZORIAN, 1966, syn. nov.

\section{Taxonomic acts}

Omalium kociani ZANETTI spec. nov. - urn:lsid:zoobank.org:act:5F24413E-6AB4-4D1C-B567-AB93C38380C9

Proteinus baculatus Assing spec. nov. - urn:lsid:zoobank.org:act:3DDB2D69-A000-4FFA-AAC7-48CE4BAB7020

Bryaxis armeniacus BRACHAT spec. nov. - urn:lsid:zoobank.org:act:01F595CB-9F3B-4DEE-8A4D-C97F39DE26E1

Bryaxis meghruicus BRACHAT spec. nov. - urn:lsid:zoobank.org:act:AA6E7E2A-4450-48E8-8048-8061C3C6DF5C

Atheta (Paralpinia) meghruica Assing spec. nov. - urn:lsid:zoobank.org:act:4673F1AA-F8C1-4524-AFA3-99E4E7F502FF

Bellatheta khustupica Assing spec. nov. - urn:lsid:zoobank.org:act:C342B3DE-9EE5-4950-85BD-817DBEE6FE3E

Calodera alticola Assing spec. nov. - urn:lsid:zoobank.org:act:3BF46F7B-FBDB-4F1B-B0D0-89D669B021C2

Tachyusa unguis Assing spec. nov. - urn:lsid:zoobank.org:act:A4B4E5C2-EEB2-4146-B6F2-27D5873D5AEB

Anotylus hamatoides SCHÜLKE, spec. nov. - urn:lsid:zoobank.org:act:0167E052-EBAB-421A-BB72-39E33C1EDC97

Euconnus (Tetramelus) longilaminatus МеҮвонм spec. nov. - urn:Isid:zoobank.org:act:96692EB3-A82F-4ADB -A278EC1B64040C82

Euconnus (Tetramelus) tavushus Меувонм spec. nov. - urn:lsid:zoobank.org:act:7F2BBD70-A7F8-4272-8195-02884B 90F2DD

Euconnus (Tetramelus) karabakhus Меувонм spec. nov. - urn:lsid:zoobank.org:act:A60B66A1-20EB-4341-9950B6485B1A3853

Neuraphes (Paraphes) gomarantsus Меүвонм spec. nov. - urn:lsid:zoobank.org:act:102F7EEC-B2A5-458F-B271E3582970195F

Neuraphes(Paraphes)syunikusMeYвонмspec. nov.-urn:lsid:zoobank.org:act:2972F28B-0968-4810-B9F5-471E93F97F43

\section{Key words}

Coleoptera, Staphylinidae, Palaearctic region, Caucasus region, Armenia, Nagorno-Karabakh, taxonomy, diversity, zoogeography, endemism, discontinuous distributions, ecology, new species, new synonyms, revalidation, new records, checklist, distribution maps.

\section{Zusammenfassung}

Über die Staphylinidenfauna von Armenien and Bergkarabach war bisher wenig bekannt. Auf Basis der Bearbeitung von mehr als 31.000 in den letzten Jahren in verschiedenen Habitaten und mit unterschiedlichen Methoden gesammelten Staphyliniden sowie einer kritischen Auswertung von Literaturnachweisen wird eine Checkliste der Fauna von Armenien und Bergkarabach zusammengestellt. Aus Armenien sind derzeit 675, aus Bergkarabach 198 Arten nachgewiesen. Trotzdem ist das Arteninventar bei weitem noch nicht vollständig erfasst. Insgesamt werden 262 Arten aus Armenien und 183 Arten aus Bergkarabach erstmals gemeldet. Frühere Nachweise von insgesamt 99 Arten beruhen wahrscheinlich auf Fehldeterminationen oder wurden irrtümlich Armenien zugeordnet. Ein Vergleich der Artenzahlen und systematischen Zusammensetzung mit den Faunen anderer Länder und Gebieten der Kaukasusregion ergab, dass die derzeit bekannte Diversität deutlich geringer als zu erwarten ist. Darüber hinaus fällt der bemerkenswert hohe Anteil (40 \%) der Aleocharinae in den Faunen von Armenien und Bergkarabach auf, wobei allein die Gattung Atheta Thomson, 1858 in Armenien 10 \% der Gesamtdiversität ausmacht. Der Artenbestand in Armenien und Bergkarabach wird vor allem durch weit verbreitete Arten geprägt, von denen viele als kaspische (kaukasische) oder iranische Faunenelemente einzuordnen sind. Nur 23 Arten, darunter 13 Aleocharinae (die meisten davon aus der Gattung Geostiba Thomson, 1858), vier Pselaphinae, fünf Scydmaeninae und eine Art der Staphylininae, werden als Regionalendemiten, eine Art der Aleocharinae vom Mount Khustup als Lokalendemit klassifiziert. Die Verbreitung einer weiteren Art ist auf das südliche Armenien und angrenzende Gebiete des Nordiran beschränkt. Eine beträchtliche Zahl von Arten ist zwar derzeit ausschließlich aus Armenien bekannt, allerdings entweder taxonomisch ungeklärt oder sehr wahrscheinlich auch in angrenzenden Ländern verbreitet. Die Nachweise einiger Arten aus Armenien und/oder Bergkarabach offenbarten eine Reihe von auffälligen Disjunktionen mit Verbreitungslücken von bis zu 2800 km; drei solcher Verbreitungsgebiete werden anhand von Karten veranschaulicht. Vierzehn Arten werden erstmals beschrieben: Omalium kociani ZANetTi spec. nov. (Armenien: Jermuk) aus der Unterfamilie Omaliinae, Proteinus baculatus Assing spec. nov. (Armenien; Nordosttürkei) (Proteininae), Bryaxis armeniacus 
Brachat spec. nov. (Armenien: NW Hrazdan) und B. meghruicus Brachat spec. nov. (Südarmenien: Meghru range) (Pselaphinae), Atheta (Paralpinia) meghruica Assıng spec. nov. (Südarmenien: Meghru-Gebirge), Bellatheta khustupica Assing spec. nov. (Südarmenien: Mount Khustup), Calodera alticola Assıng spec. nov. (Armenien: Mount Karkar) und Tachyusa unguis Assing spec. nov. (Südarmenien) der Aleocharinae, Anotylus hamatoides SchülKe spec. nov. (Armenien) der Oxytelinae, Euconnus (Tetramelus) longilaminatus Меувонм spec. nov. (Nordarmenien), E. (T.) tavushus Меувонм spec. nov. (Nordarmenien), E. (T.) karabakhus Меувонм spec. nov. (Bergkarabach), Neuraphes (Paraphes) gomarantsus Меувонм spec. nov. (Südarmenien: Meghru-Gebirge) und N. (P.) syunikus Мечвонм spec. nov. (Südarmenien) der Scydmaeninae. Acht Namen werden synonymisiert und einer revalidiert: Dialycera minuta Luze, 1906 = Phyllodrepa armena Iablokoff-Khnzorian, 1959, syn. nov.; Mycetoporus silvaticus IAblokoff-Khnzorian, $1962=$ M. dispersus SCHÜlKe \& Kocian, 2000, syn. nov.; Aleochara subtumida $($ Hochнuтн, 1859) = Aleochara khnzoriani Amiryan, 1999, syn. nov.; Platystethus cephalotes Eppelsheim, 1878, revalidiert (vorher Synonym von P. laevis Märkel \& Kiesenwetter, 1848) = P. oblongopunctatus Roubal, 1911, syn. nov.; Euconnus lalvarensis IABLoKoff-Khnzorian, 1964 = Euconnus pseudorobustus Franz, 1986, syn. nov.; Astenus rufopacus Reitter, 1909 = A. baali Coiffait, 1960, syn. nov.; Heterothops dissimilis (Gravenhorst, 1802) $=$ H. armeniacus CoIfFAIT, 1977, syn. nov.; Heterothops praevius ERICHSON, $1839=$ Heterothops montanus IABLOKOFF-KHNZORIAN, 1966, syn. nov.

\section{Schlüsselwörter}

Coleoptera, Staphylinidae, Paläarktis, Kaukasusregion, Armenien, Bergkarabach, Taxonomie, Diversität, Zoogeographie, Endemismus, Ökologie, neue Arten, neue Synonymien, Erstnachweise, Checkliste, Verbreitungskarten

\section{Introduction}

Its area covering a mere $29,800 \mathrm{~km}^{2}$, Armenia is a small country in the northeast of the Armenian Highlands and to the south of the Lesser Caucasus. It borders on Georgia in the north, Turkey in the west, the Azerbaijanian exclave Nakhchivan in the southwest, Iran in the south, and Azerbaijan and the Republic of Artsakh (NagornoKarabakh) in the east. Most of the area is dominated by mountains and high plateaus, with a mean altitude of approximately $1800 \mathrm{~m}$, the highest peak (Mount Aragats) at $4090 \mathrm{~m}$, numerous summits at more than $3000 \mathrm{~m}$, and the lowest elevation at $380 \mathrm{~m}$ in the Aras river valley in the south.

Nagorno-Karabakh, officially named Republic of Artsakh since 2017, is a de facto independent state. It formed part of Azerbaijan prior to the Nagorno-Karabakh War and is still recognized as such by the UN today. Its small territory of barely $11,500 \mathrm{~km}^{2}$ shares borders with Azerbaijan in the north and east, with Armenia in the west, and Iran in the south. Like Armenia, Nagorno-Karabakh is mostly covered by mountains and highlands, with an average altitude of approximately $1100 \mathrm{~m}$ and with two major mountain ranges, the Mrav range in the north (highest peaks Gomshasar and Mrav at 3720 and $3340 \mathrm{~m}$, respectively) and the Karabakh range in the west with several peaks at above $2500 \mathrm{~m}$.

The mountainous topography and geology of the entire Armenian Highland, which includes the territories of Armenia and Nagorno-Karabakh, was primarily shaped by the collision of the Arabian with the Eurasian plate. Since the Arabian plate is moving northwards at $2.5 \mathrm{~cm}$ per year even today (NeukiRCHEN 2011), the Armenian Plateau is still tectonically and seismically active, the last major earthquake dating back only to 1988 . The geological history also explains why the region is characterized by a mix of various mesozoic and palaeogenic sediments, neogenic continental sediments, and of volcanic rocks of various ages (PlöCHINGER 1979).

According to Fauyvush \& Aleksanyan (2016), four major geomorphological regions can be distinguished in Armenia. They are the mountains and valleys in the northeast, which have been subject to massive erosion, areas covered by lava of Pliocene origin not significantly affected by erosion, ridged mountains in the south with signs of heavy erosion, and the Ararat valley with a cover of alluvial and proluvial sediments.

The climate is essentially dry continental, with dry and hot summers and with cold winters. However, pronounced gradients in temperature and precipitation can be observed along different altitudinal regimes, the mean annual temperature varying between 2.7 and $14.1^{\circ} \mathrm{C}$ and the mean annual precipitation between 250 (Ararat plain) and $1000 \mathrm{~mm}$ (Mount Aragats).

As a result of the diversity of topography, geology, and climate in the region, Armenia (and Nagorno-Karabakh) are shaped by a wide range of landscapes such as desert (Transcaucasian sand desert), semi-deserts, and salt marshes (at altitudes of 480-1200 m), steppes and forests at altitudes of $1200-2200 \mathrm{~m}$, as well as subalpine and alpine meadows, carpets, and wetlands at 2200$4000 \mathrm{~m}$. Approximately 3800 species of vascular plants, 428 species of algae, 399 species of mosses, 4207 species of fungi, 464 species of lichens, and 549 species of vertebrates have been recorded from Armenia, with the flora including 142 endemic plant species (FAuYvush \& 
Aleksanyan 2016). According to the website of the Office of the Nagorno Karabakh Republic (www.nkrusa.org), the flora of Nagorno-Karabakh is composed of 2000 probably vascular - plant species.

The Armenian forest habitats are composed of 110 tree and 152 shrub species. The dominant tree species are Quercus spp., Fagus orientalis, and Carpinus betulus, which alone account for approximately $81 \%$ of the forest cover. The remainder is mostly composed of pine (mostly secondary), juniper, hornbeam coppice, lime, ash, and maple. The forest cover has significantly decreased, primarily due to deforestation, from $35 \%$ to $7-8 \%$ during the past 6000 years. However, large areas especially in Northwest, West, and Central Armenia have not supported forests since the Tertiary period. Today, $62 \%$ of the remaining forested areas are found in the northeast, $36 \%$ in the southeast, and only $2 \%$ in the central parts of Armenia (Moreno-SANCHEZ \& SAYADYAN 2005). The western and northern parts of Nagorno-Karabakh, by contrast, still feature extensive primary forests.

According to Fauyvush \& Aleksanyan (2016), the Armenian invertebrate fauna is composed of 17200 species. However, this figure is probably based on rough estimates rather than long-term modern studies and most likely requires revision. At least this can be said for the Staphylinidae (rove beetles), the most speciose of all beetle families and in fact the most speciose of all families of organisms. The two editions of the Palaearctic Catalogue (Smetana 2004, Schülke \& Smetana 2015) list 340 and 395 species, respectively, for Armenia. No such data are available for Nagorno-Karabakh, since it was treated as a part of Azerbaijan in these works. However, even the recent figures for Armenia are not only incomplete, but also misleading and largely erroneous, primarily because of the changing interpretations, definitions, and boundaries of the geographic term "Armenia" over the centuries. Most of what was regarded as Armenia and East Armenia in the 19th century belongs to Turkey, Georgia, or Azerbaijan today. The same is true of the geographic terms "Hochland von Armenien" (Armenian Highland) and "Armenisches Gebirge" (Armenian Mountains), the latter of which includes the Lesser Caucasus and adjacent mountain ranges such as the Pontic Mountains. Moreover, numerous species reported from the historical locality "Araxestal" (or equivalent) (e.g., Eppelsheim 1890a, b) have erroneously been referred to Armenia. This German term was used for what is Ordubad in the Azerbaijanian exclave Nakhchivan today and at the same time denotes "Aras river valley". The Aras river forms the border between Turkey and Armenia, as well as that between Iran and Armenia and Azerbaijan, respectively.

By around 1830, the regions of the Caucasus and Transcaucasica were under Russian administration. In the following period, this area was visited by several coleopterists such as Ménétriés (1827-31), Scovicz (1827-1830), Motschulsky (1834-35, 1837-38), Kolenati
(1843-45), Chaudoir (1845), and Gotsch (1845). At least Kolenati (see Kolenati 1858: $32 \mathrm{ff}$.), Motschulsky (see Motschulsky 1850: 18 ff.), Gotsch (see HochHUTH 1847: 449), and probably also Scovicz (see Motschulsky 1850: 19, FAldermanN 1837: $42 \mathrm{f}$.) collected material in the region that is Armenia or Nagorno-Karabakh today.

The first descriptions of Armenian Staphylinidae were probably published by FALDERMANN (1835), who studied the material collected by Scovicz, in his "Coleoptera Persico-Armeniaca". However, since he did not specify localities in this work, the exact origin of the type material is unknown. Kolenati (1846) reported eleven species of Staphylinidae, six of them newly described, from "Karabagh" and eight species, five of them new, from Armenia, but only the localities of one of these species are in Armenia today. Носннuтн (1849) described numerous species of Staphylinidae, mostly without specifying localities, based on material from Chaudoir, Gotsch, and Motschulsky. In some cases he indicated Armenia as the origin, but it is rather unlikely that the respective localities are within the boundaries of modern Armenia (see above). In the second half of the $19^{\text {th }}$ century, the German entomologist Hans Leder significantly contributed to the knowledge of the beetle fauna of the Caucasus region by conducting long-term collecting expeditions in the years $1875-1881$. From 1882 to 1888 he lived in Helenendorf (today Göygöl in Azerbaijan). His earlier expeditions (1875-1879) did not, however, include the territory of modern Armenia (SCHNEIDER \& LEDER 1878: $23 \mathrm{ff}$., LEDER 1880: $451 \mathrm{ff} ., 1881: 501 \mathrm{ff}$.), and his later collecting activities focused on the environs of Ordubad (Hetschko 1922). In 1875, Schneider (see Schneider \& LEDER 1878: 6 ff.) collected beetles in North and Central Armenia (Gyumri, Yerevan, Vagharshapat). His Staphylinidae were studied by EPPELSHEIM (1878), who reported eleven species from Armenia, one of them new. In the early $20^{\text {th }}$ century (1915-1916), the Polish entomologist W. Eichler collected beetles in the environs of Vagharshapat. The list of species published by EICHLER (1930) includes 87 species of Staphylinidae.

In the second half of the $20^{\text {th }}$ century, it was S. M. Iablokoff-Khnzorian who significantly contributed to the knowledge of Armenian beetles, including rove beetles. In total, he described 28 new species of Staphylinidae from Armenia (IABLOKOFF-KHNZORIAN 1956, 1957b, 1959, 1960, 1961, 1962, 1964a, 1966, 1989). Besides, he actively engaged in a collaboration with other specialists who described new species and published records based on his Armenian material, e.g., BENICK (1974), Coiffait $(1965,1966 a, b, 1967 a, b, 1968,1970 a$, b, d, 1972a, 1977), Likovsky (1971), PACE (1982, 1983), and Ullrich (1975). Around the turn of the millenium, Amiryan (1999a, b) and Amiryan \& al. (2000) published three articles on the genus Tachinus, a new species of Aleochara, and the pholeophilous, nidicolous, and myrmecophilous Staphylinidae of Armenia. 
Extensive additional information is scattered in numerous articles, especially comprehensive taxonomic revisions, not specifically dealing with the fauna of Armenia. These include Ryvkin (1990: Stenus), Sabella \& al. (2004: Brachygluta), Assing (2005b: Leptobium; 2007b: Pronomaea; 2008c: Sunius; 2010a: Luzea; 2010b: Achenium; 2016a: Anaulacaspis; 2018e: Cousya), Jászay \& Hlaváč (2006: Dropephylla), and Enushchenko \& Semenov (2016: Gyrophaena), to name only a few. For additional articles see Tab. 1, 3 and the reference section in this paper.

The present study aims at providing a revised overview and a first critical checklist of the Staphylinidae of Armenia and Nagorno-Karabakh. It is primarily based on the results of three field trips conducted by the authors in June/July in three consecutive years (2016-2018), the first two exclusively to Armenia and the third trip to both Nagorno-Karabakh and Armenia. In addition, material from field trips to Armenia conducted by Matús Kocian in 2015 and by Jörg Müller and Andrea JarzabekMüller in 2016 was examined. The staff of the Zoological Institute in Yerevan, including Mark Kalashian, Tigran Ghrejyan, and Gayane Karagyan, provided substantial material from pitfall trap studies in Armenia. Harald Schillhammer contributed specimens and data of material collected in Armenia in 2001, together with Helena Shaverdo. Finally, a number of additional specimens located in various museum and private collections is included.

Some results of the three field trips conducted by the authors have already been published in separate articles: Assing (2016b-c, 2017b-d, 2018a-c, g-h), Assing \& Vogel (2017), and Schülke (2019a-b). Results of revisions of type material in the Iablokoff-Khnzorian collection have been addressed by Assing (2005a, 2017a, 2019a, b).

\section{Material and methods}

The material treated in this study is deposited in the following public and private collections:

HNHM Hungarian Natural History Museum, Budapest (Gy. Makranczy)

IZAY Armenian National Academy of Sciences, Institute of Zoology, Yerevan (M. Kalashian, T. Ghrejyan, G. Karagyan)

MCSNV Museo Civico di Storia Naturale di Verona (L. Latella)

MNB Museum für Naturkunde Berlin (J. Frisch)

MZH Finnish Museum of Natural History, Zoological Museum, Helsinki (J. Mattila, J. Muona)

NHMW Naturhistorisches Museum Wien (H. Schillhammer)

NMP National Museum of Natural History, Praha (J. Hájek)

cAss private collection Volker Assing, Hannover
cBra private collection Volker Brachat, Geretsried cFel private collection Benedikt Feldmann, Münster cKhn Khnzorian collection (currently in private collection Mark Kalashian, Yerevan)

cKoc private collection Matúš Kocian, Prague

cMey private collection Heinrich Meybohm, Großhansdorf

cPut private collection Volker Puthz, Schlitz

cSch collection M. Schülke (MNB)

cWun private collection Paul Wunderle, Mönchengladbach

cZan private collection Adriano Zanetti, Verona

Most of the material collected by the authors is housed in cAss and cSch; the Pselaphinae and Scydmaeninae collected by the first author are deposited in $\mathrm{cBra}$ and cMey, respectively, reference material of other groups also in MNB, NHMW, cFel, and cPut. With few exceptions in cAss, cPut, and cSch, specimens found by Harald Schillhammer and Helena Shaverdo are in NHMW. The material from the field trip conducted by M. Kocian is mainly in cKoc, partly also in cAss and cSch. The trap catches made available by J. Müller and T. Ghrejyan is deposited mostly in cAss, partly in IZAY, MNB, and cSch.

Body length was measured from the anterior margin of the mandibles (in resting position) (most subfamilies) or the anterior margin of the clypeus (Tachyporinae, Oxytelinae) to the posterior apex of the elytra (Scydmaeninae) or to the abdominal apex (other subfamilies), the length of the forebody from the anterior margin of the mandibles (in resting position) to the posterior margin of the elytra, head length along the middle from the anterior margin of the clypeus (without ante-clypeus) (Aleocharinae) or from the anterior margin of the frons to the posterior constriction of the head (other subfamilies), elytral length at the suture from the apex of the scutellum to the posterior margin of the elytra (if not indicated otherwise), and the length of (the median lobe of) the aedeagus from the apex of the ventral process to the base of the aedeagal capsule. The "parameral" side (i.e., the side where the sperm duct enters) is referred to as the ventral, the opposite side as the dorsal aspect.

The maps were created using Map Creator 2.0 (primap) software. Zoogeographic terminology is primarily based on LATTIN (1967).

Labels of type material are cited in the original spelling, except that geographic and other data given in Cyrillic script are transliterated. Different labels are separated by slashes.

The taxonomic parts on Omaliinae (section 3.6.1), Pselaphinae (section 3.6.2) and Scydmaeninae (section 3.6.7) are exclusively authored by Adriano Zanetti, Volker Brachat, and Heinrich Meybohm, respectively, those on Tachyporinae and Oxytelinae (sections 3.6.4, 3.6.6) by the second author, and the remainder (sec tions 3.6.2, 3.6.5, 3.6.8, 3.6.9) by the first author. 


\section{Results and discussion}

\subsection{Diversity}

During the three field trips to Armenia and NagornoKarabakh conducted by the authors in 2016-2018, as many as 28,927 specimens of Staphylinidae were collected, 20,016 specimens in Armenia and 8,911 in Nagorno-Karabakh. Significant additional material from Armenia, approximately 2,000 specimens (or data of specimens), was contributed by Harald Schillhammer (hand-collected), Jörg Müller (hand-collected), Matúš Kocian (hand-collected), Mark Kalashian, Tigran Ghrejyan, and Gayane Karagyan (pitfall traps). Finally, some specimens collected by various collectors and deposited in several museums and private collections was studied. Hence, the present monograph is based on a total of approximately 31,000 specimens from numerous localities distributed across practically the whole of Armenia (Map 1).
In total, 525 named species were identified at the species level (Tab. 1). (To allow for better readability, the two subspecies of Bolitobius castaneus are treated as species in this section.) Approximately 1,900 specimens of Mocyta Mulsant \& Rey, 1874 (currently under revision) and some 150 other Staphylinidae representing an unknown number of additional species were not named (see Tab. 2).

Disregarding doubtful or erroneous literature records of 99 species (see Tab. 3), 689 species in 15 subfamilies are currently known from Armenia and Nagorno-Karabakh, 675 from Armenia and 198 from Nagorno-Karabakh. As many as 262 species (39\% of total diversity) are reported from Armenia and 184 (92\%) from NagornoKarabakh for the first time (Tab. 1). Atheta aequicollis, a species previously known only from Mongolia, is even newly reported from the West Palaearctic region. Given the situation in 2016, the number of first records would be even significantly greater. However, the material of several taxa (Mycetoporus Mannerheim, 1830; Sepedo-

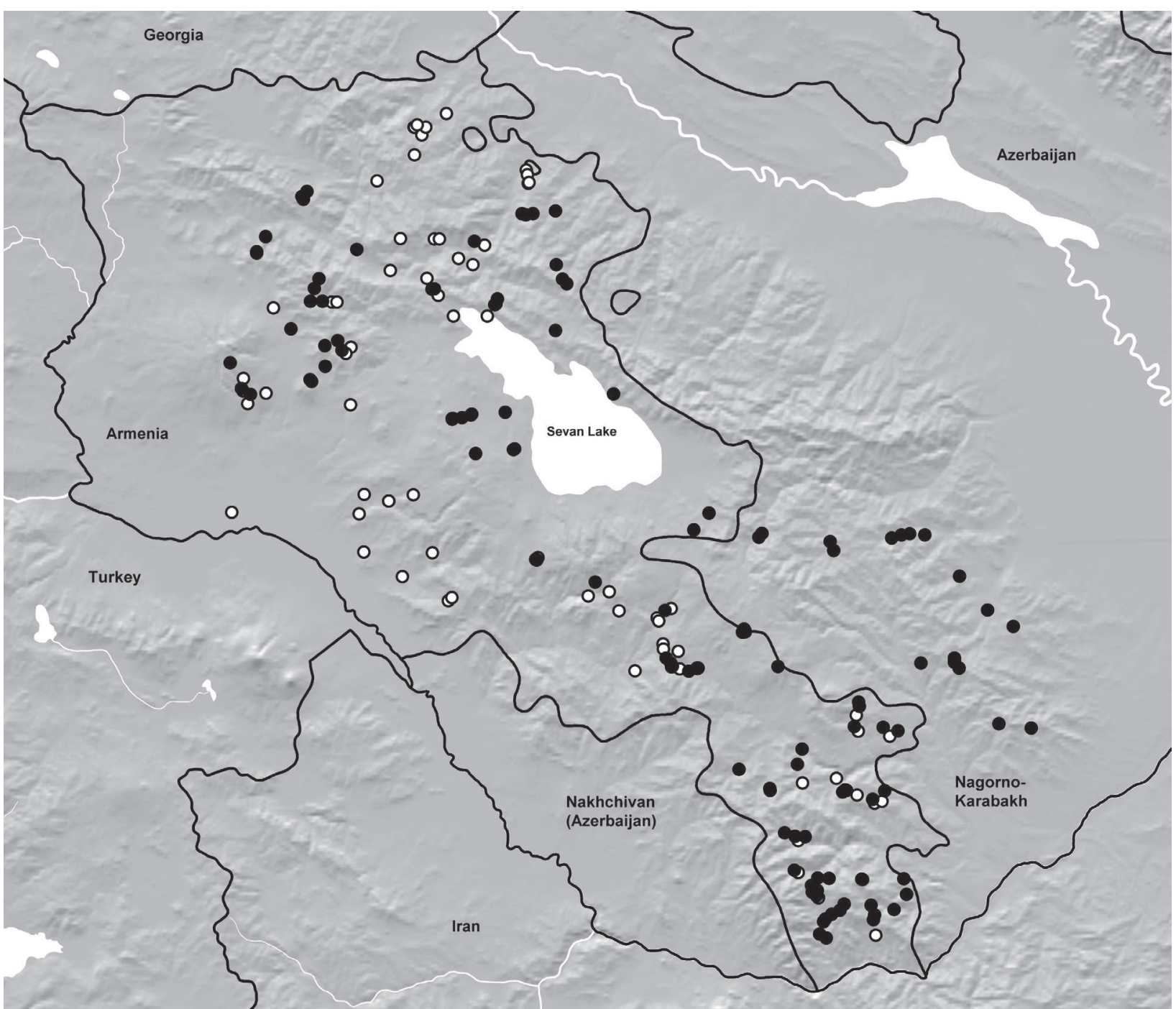

Map 1: Sampling localities in Armenia and Nagorno-Karabakh. Black circles: authors' field trips (localities 1-131); white circles: localities 132-213 (see Tab. 1). 
philus Gistel, 1856; Atheta Thomson, 1858; Geostiba Thomson, 1858; Leptusa KraAtz, 1856; Liogluta Thomson, 1858; Myllaena Erichson, 1837; Oxypoda Mannerheim, 1830; Lobrathium Mulsant \& Rey, 1878; Quedius Stephens, 1829) has already been treated and published in separate articles, including the descriptions of 28 new species, the vast majority of them in the genera Geostiba (nine species), Atheta (seven species), and Oxypoda (six species) (Assing 2016b, c, 2017a-c, e, 2018a, c, g, h, Assing \& Vogel 2017, Schülke 2019a, b). Fourteen additional species - five of Scydmaeninae, four of Aleocharinae, two of Pselaphinae, and one each of Omaliinae, Proteininae, and Oxytelinae - are newly described in the present paper (see section 3.6), and the description of one species of Staphylininae is in preparation (SCHILlhammer in prep.).

With a total of 260 species (38\%), the Aleocharinae are, by far, the subfamily with the greatest diversity in the study region; in Armenia, they alone account for 254 (38\%) species and for 123 (47\%) first records, in Nagorno-Karabakh for 78 (39\%) species and 73 (40\%) first records. Other highly diverse subfamilies are the Staphylininae with 98 species in the study region (Armenia: 95; Nagorno-Karabakh: 30), followed by the Tachyporinae (total: 59 species; Armenia: 59; NagornoKarabakh: 28), the Oxytelinae (total: 59; Armenia: 58; Nagorno-Karabakh:9), the Steninae (total: 50; Armenia: 50; Nagorno-Karabakh: 12), the Paederinae (total: 47; Armenia: 47; Nagorno-Karabakh: 8), the Omaliinae (total: 44; Armenia: 43; Nagorno-Karabakh: 12), and the Pselaphinae (total: 41; Armenia: 41; Nagorno-Karabakh: 7). The relative representation of first records in these subfamilies is primarily related to how well the respective groups and regions have been studied. In the Staphylininae, the new records account for $23 \%$ of the diversity in Armenia and $97 \%$ in Nagorno-Karabakh, in the Tachyporinae for $42 \%$ in Armenia and $89 \%$ in Nagorno-Karabakh, in the Oxytelinae for $42 \%$ in Armenia and $78 \%$ in Nagorno-Karabakh, in the Steninae for $18 \%$ in Armenia and $75 \%$ in Nagorno-Karabakh, in the Paederinae for $21 \%$ in Armenia and $100 \%$ in NagornoKarabakh, in the Omaliinae for $47 \%$ in Armenia and $83 \%$ in Nagorno-Karabakh, and in the Pselaphinae for $20 \%$ in Armenia and $86 \%$ in Nagorno-Karabakh.

The evident differences between the figures for Armenia and Nagorno-Karabakh are explained by the fact that the latter region is significantly smaller, less diverse, that next to nothing was known about its Staphylinidae fauna previously, and that it was sampled for only ten days in summer 2018.

A comparison of the total diversity and the systematic composition of the currently known Staphylinidae faunas of Armenia and Nagorno-Karabakh with those of the neighbouring or geographically close Georgia, the Russian South European territory, and Iran reveals some unsurprising similarities and differences, but also conspicuous discrepancies. Considering the much smaller territory, it was to be expected that Armenia and particularly Nagorno-Karabakh host fewer Staphylinidae species than any of the other regions. On the other hand, the data show that the fauna of Georgia and especially that of Iran have been very poorly studied. Based on preliminary studies and on revisions of certain genera such as Geostiba, Leptusa, and Ischnosoma (Assing 2016b, 2017b, c, Assing \& SCHülke 2017), the western parts of Georgia are rich in endemic species, and there is no reason to assume that even the eastern parts are less diverse than Armenia. Moreover, the area of Georgia exceeds that of Armenia by a factor of approximately 2.3. Based on these observations, it can be inferred that the difference in diversity between Georgia and Armenia should be significantly greater than that indicated in Tab. 4. And Iran, with its vast territory of about 55 times the size of Armenia and 144 times the size of NagornoKarabakh and with its topological, geological, and climatic diversity should be expected to host at least twice as many species as Armenia and Nagorno-Karabakh combined. The high proportion of paederines and the low percentage of omaliines in Iran compared to the figures for the other countries and regions is explained with its more southern geographic position; paederines tend to be more, and omaliines less speciose in warmer climates.

Another remarkable observation is that the proportions of aleocharine species in Armenia and Nagorno-Karabakh are about 1.5 times as great as that in Georgia and about twice as great as those in the Russian South European territory and in Iran. The most speciose and at the same time believed to be the most difficult to identify and consequently the least studied of all subfamilies (with the lowest number of active specialized taxonomists), the Aleocharinae of Armenia and Nagorno-Karabakh can now be considered to be better known than those of other countries of the Caucasus region sensu lato. With 67 identified and an unknown number of unidentified species, the genus Atheta is particularly diverse in Armenia and alone accounts for approximately $10 \%$ of the total diversity.

A general conclusion that can be drawn from the data in Tab. 4 is that the known species inventories of all Caucasian countries and regions are far from complete. This is revealed when the species numbers are compared to those of small countries like Denmark (area only slightly greater than that of Armenia; 1045 species) and Belgium (area even smaller than that of Armenia; 1303 species) without endemics, with a much lower habitat diversity (e.g., without mountains), and with a history of glacial devastation on the one hand, but a long entomological tradition on the other. Long-term studies, including thorough sampling of certain habitats such as dead wood, decaying matter of all kinds (compost, dung, etc.), riparian and other wetland habitats, nests of ants, mammals, and birds, and especially also using methods suitable for collecting flying insects (car-nets, window traps) or for recording species with short epigeic dispersal activity (pitfall traps) will undoubtedly result in a 
significant increase of species numbers. This is particularly true of the fauna of Nagorno-Karabakh, whose fauna is still characterized by pronounced gaps in all subfamilies, particularly in the diversities of Oxytelinae and Pselaphinae.

\subsection{Checklist}

The below checklist (Tab. 1) summarizes the results of various field trips to Armenia and one to NagornoKarabakh, pitfall and window trap studies in Armenia, identification of additional material from other collections, as well as previous literature records. In the localities column, the number of specimens is given in parentheses behind the locality/sample number (or letter). For most of the species that had been recorded from Armenia and Nagorno-Karabakh prior to the present study, primary records are indicated in the references column. Secondary catalogue records (e.g., Herman 2001, Löbl \& Besuchet 2004, Schülke \& SMetana 2015) or general records provided in keys are included only when primary records were not found. In some cases (especially Steninae and Pselaphinae), such catalogue records are in fact the primary records. Literature records that are at least likely to be based on misidentification, old records of species that are reliably identified only based on an examination of the primary sexual characters, records (most likely) erroneously attributed to the territory of modern Armenia, and records that are doubful for other reasons are discarded and listed in Tab. 3. Some doubtful literature records were included, however, when the presence of a species in Armenia and/or Nagorno-Karabakh was confirmed based on recently collected material. Recently collected material that could not be reliably identified at the species level for various reasons is listed separately in Tab. 2.

Tab. 1: Checklist of the Staphylinidae recorded from Armenia and Nagorno-Karabakh. For footnotes and details on the localities, samples, and references see the respective sections below the checklist.

Symbols and abbreviations: 0 : first record(s) from Armenia or Nagorno-Karabakh, respectively; $\bigcirc$ : previously recorded from Armenia or Nagorno-Karabakh, respectively; \#: recorded only from Armenia, but taxonomic and zoogeographic status doubtful; *: recorded only from Armenia, but probably more widespread; ${ }^{* *}$ : regionally endemic (distribution most likely confined to particular regions or mountain ranges within Armenia and/or Nagorno-Karabakh; ${ }^{* *}$ locally endemic to individual mountains. $\mathrm{AR}=$ Armenia; NK = Nagorno-Karabakh.

\begin{tabular}{|c|c|c|c|c|}
\hline Species & AR & NK & Localities/Samples & References \\
\hline \multicolumn{5}{|l|}{ Omli in a e } \\
\hline $\begin{array}{l}\text { Anthobium atrocephalum } \\
\text { (GYLLENHAL, 1827) }\end{array}$ & 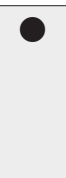 & O & $\begin{array}{l}22(9), 33(1), 39(2), 63(1), 66 b(1), 70(4), 72(2), \\
89 b(2), 100(1) 105(2), 108(4), 110(2), 112(3), \\
113(5), 114(27), 121 a(1), 122(30), 126(3), 133(1), \\
135(1), 136(1), 151(1)\end{array}$ & \\
\hline $\begin{array}{l}\text { Anthobium fusculum (ERICHSON, } \\
\text { 1839) }\end{array}$ & $\bigcirc$ & & 122(105), 165(3) & A05a, IK61 ${ }^{6)}$ \\
\hline Anthobium hamatum (LuzE, 1905) & O & 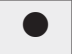 & $70(1), 114(1)$ & L05 \\
\hline $\begin{array}{l}\text { Anthobium tenue (EPPELSHEIM, } \\
\text { 1881) }\end{array}$ & 0 & & $170(6)$ & \\
\hline $\begin{array}{l}\text { Anthobium unicolor (MARSHAM, } \\
\text { 1802) }\end{array}$ & 0 & & $181(1)$ & \\
\hline $\begin{array}{l}\text { Arpedium brachypterum (GRAVEN- } \\
\text { HORST, 1802) }\end{array}$ & 0 & & $67(31)$ & \\
\hline Dialycera minuta Luze, 1906 & $\bigcirc$ & & & IK59 ${ }^{60)}$ \\
\hline $\begin{array}{l}\text { Dropephylla caucasica (KolenAti, } \\
1846 \text { ) }\end{array}$ & O & 0 & $42(3), 48(20), 49(2), 49 a(3), 53(1), 116(2)$ & ATHW0, IK64b \\
\hline $\begin{array}{l}\text { Dropephylla elisabethae JÁszAY \& } \\
\text { HLAvÁč, } 2006\end{array}$ & O & & & JH06 \\
\hline Dropephylla vilis (ERICHSON, 1840) & $\bigcirc$ & & $30(1), 121 \mathrm{a}(1)$ & J08 \\
\hline Eusphalerum celsum (Luze, 1910) ${ }^{1)}$ & O & & $8(1), 68(1), 68 \mathrm{a}(1)$ & \\
\hline Eusphalerum fidele (LuzE, 1910) ${ }^{1)}$ & ○ & & $5 \mathrm{a}(6), 6(2), 7(8), 11(2), 53(1), 63(2), 75(1), 97(1)$ & \\
\hline $\begin{array}{l}\text { Eusphalerum primulae (STEPHENS, } \\
1834 \text { ) }\end{array}$ & 0 & & $135(1), 136(2)$ & \\
\hline
\end{tabular}




\begin{tabular}{|c|c|c|c|c|}
\hline Species & AR & NK & Localities/Samples & References \\
\hline $\begin{array}{l}\text { Eusphalerum sareptanum (EPPELS- } \\
\text { HEIM, 1878) }\end{array}$ & O & O & $114(1)$ & Z93 \\
\hline $\begin{array}{l}\text { Eusphalerum sorbi (GYLLENHAL, } \\
1810 \text { ) }\end{array}$ & 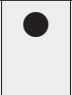 & & 6(3), 7(2), 8(2), 69(1) & \\
\hline $\begin{array}{l}\text { Eusphalerum transcaucasicum } \\
\text { (BERNHAUER, 1902) }\end{array}$ & O & 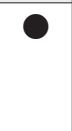 & $\begin{array}{l}30(1), 31(3), 33(11), 53(3), 58(2), 59(6), 66(3), \\
66 a(1), 71(1), 96(1), 100(2), 101(2), 102(2), 105(8) \\
106(4), 119(4)\end{array}$ & Z93 \\
\hline $\begin{array}{l}\text { \#Geodromicus armeniacus IABLO- } \\
\text { KOFF-KHNZORIAN, } 1989\end{array}$ & O & & & IK89 \\
\hline $\begin{array}{l}\text { Geodromicus brevicollis FAUVEL, } \\
1857\end{array}$ & 0 & & $\begin{array}{l}\text { 18(3), 45(2), 46(10), 49(25), 67(18), 85(25), 85a(24), } \\
85 \mathrm{c}(8), 86(3), 94(1), 128(5), \mathrm{j}(2), \mathrm{k}(1), \mathrm{l}(1)\end{array}$ & ASpp \\
\hline $\begin{array}{l}\text { Geodromicus constricticollis EPPELS- } \\
\text { HEIM, } 1890\end{array}$ & O & & & IK62 \\
\hline $\begin{array}{l}\text { Geodromicus convexus IABLOKOFF- } \\
\text { KHNZORIAN, } 1962\end{array}$ & $\bigcirc$ & & & IK62 \\
\hline $\begin{array}{l}\text { Geodromicus major (MoTsCHUL- } \\
\text { SKY, 1860) }\end{array}$ & O & & $\mathrm{m}(1)$ & \\
\hline $\begin{array}{l}\text { \#Geodromicus rivularis IABLO- } \\
\text { KOFF-KHNZORIAN, } 1962\end{array}$ & O & & & IK62 \\
\hline $\begin{array}{l}\text { Geodromicus striatus IABLOKOFF- } \\
\text { KHNZORIAN, } 1962\end{array}$ & $\bigcirc$ & & & IK62 \\
\hline $\begin{array}{l}\text { Lesteva longoelytrata longoelytrata } \\
\left(\text { GoEZE, 1777) }{ }^{1)}\right.\end{array}$ & O & & 18(7), 49(15), 63(1), 67(1), 76(2), 77(1), 139(14) & \\
\hline $\begin{array}{l}\text { Lesteva monticola KIESENWETTER, } \\
1847\end{array}$ & 0 & & $204 \mathrm{a}(1), 213(3)$ & \\
\hline Lesteva punctata ERICHsON, 1839 & 0 & 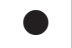 & $20 \mathrm{a}(1), 63(5), 66 \mathrm{a}(8), 73(1), 107(3), 112(8), 130(7)$ & \\
\hline $\begin{array}{l}\text { Mannerheimia brevipennis (МоT- } \\
\text { SCHULSKY, 1860) }\end{array}$ & O & & $11 b(1)$ & A05a, IK56 ${ }^{7)}$ \\
\hline $\begin{array}{l}\text { *Olophrum aragatzense } \\
\text { IABLOKOFF-KHNZORIAN, } 1962\end{array}$ & $\bigcirc$ & & $49(2), 67(82)$ & A05a, IK62 \\
\hline $\begin{array}{l}\text { Olophrum caucasicum FAUVEL, } \\
1875\end{array}$ & 0 & 0 & $\begin{array}{l}7(2), 8(1), 9(1), 22(7), 36(2), 66(1), 98(4), 112(1) \\
113(1), 114(1), 115(1), 116(8), 121 \mathrm{a}(1), 126(1)\end{array}$ & \\
\hline $\begin{array}{l}\text { Olophrum puncticolle EPPELSHEIM, } \\
1880\end{array}$ & $\bigcirc$ & & & $\begin{array}{l}\text { A05a }{ }^{2)}, A 17 a, \\
\text { IK64a }\end{array}$ \\
\hline $\begin{array}{l}\text { Omalium caesum GravENHORST, } \\
1806\end{array}$ & O & 0 & $\begin{array}{l}8(2), 9(37), 19(1), 41(1), 42(5), 48(38), 49(13), \\
50(7), 63(1), 67(3), 76(13), 77(2), 81(7), 81 a(8), \\
82(1), 82 a(1), 83(1), 105(6), 114(16), 118(1), 126(6) \\
136(2), 149 a(1), 165(1)\end{array}$ & E30 \\
\hline $\begin{array}{l}\text { *Omalium kociani ZANETTI, } \\
\text { spec. nov. }\end{array}$ & O & & $165(9)$ & \\
\hline Omalium littorale KraAtz, 1857 & ○ & $\bigcirc$ & $\begin{array}{l}4(2), 7(2), 9(85), 9 a(9), 16(1), 19(1), 22(83), 23(1), \\
30(27), 31(7), 33(1), 35(1), 39(6), 41(1), 42(3), \\
48(80), 49(17), 49 a(44), 54(33), 55(1), 57(17), \\
60(2), 64(1), 67(3), 70(7), 76(100), 81(1), 88(2), \\
89(8), 89 b(21), 91(1), 95(1), 107(1), 108(1), 113(3), \\
114(38), 116(24), 117(1), 118 a(1), 133(2), 135(1), \\
136(3), 170(5), 172(2)\end{array}$ & $\mathrm{Za02}$ \\
\hline
\end{tabular}




\begin{tabular}{|c|c|c|c|c|}
\hline Species & AR & NK & Localities/Samples & References \\
\hline Omalium rivulare (PAYKULL, 1789) & $\bullet$ & 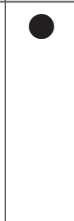 & $\begin{array}{l}1(1), 8(1), 9(8), 11(2), 11 \mathrm{~b}(2), 12(1), 13(1), 33(3), \\
39(4), 41(1), 42(1), 48(13), 49(5), 63(6), 67(2), \\
74(2), 81(3), 81 \mathrm{a}(2), 82(5), 93(1), 114(3), 116(3), \\
133(3), 135(10), 136(23), 148(1), 149 \mathrm{a}(3), 149 \mathrm{~b}(1), \\
150(13), 151(44), 155(3), 167(1), 170(2), 172(2)\end{array}$ & \\
\hline $\begin{array}{l}\text { Omalium rugatum Mulsant \& } \\
\text { REY, } 1880\end{array}$ & & $\odot$ & $105(6)$ & \\
\hline Omalium turcicum SMETANa, 1967 & $\bullet$ & & $\begin{array}{l}4(1), 7(10), 13(3), 15(1), 33(3), 39(9), 82(2), 121 \mathrm{a}(2), \\
126(8), 131(5), 165(4)\end{array}$ & \\
\hline $\begin{array}{l}\text { Omalium wunderlei ZANETTI, } \\
2002\end{array}$ & $\bullet$ & & $122(22)$ & \\
\hline $\begin{array}{l}\text { Phloeonomus minimus (ERICHSON, } \\
\text { 1839) }\end{array}$ & $\bullet$ & & $148 \mathrm{~d}(1)$ & \\
\hline $\begin{array}{l}\text { Phloeonomus punctipennis Тном- } \\
\text { soN, } 1867\end{array}$ & $\bullet$ & & $68 \mathrm{a}(1)$ & \\
\hline Phloeostiba plana (PAYKULL, 1792) & O & & 135(11), 148b(6), 148c(1), 149b(3), 150(2), 156(4) & ATHW00 \\
\hline $\begin{array}{l}\text { Phyllodrepa floralis (PAYKULL, } \\
1789 \text { ) }\end{array}$ & O & $\odot$ & $50(1), 114(2)$ & E30 \\
\hline $\begin{array}{l}\text { Phyllodrepa nigra (GRAVENHORST, } \\
\text { 1806) }\end{array}$ & $\bullet$ & & $68(1)$ & \\
\hline $\begin{array}{l}\text { Xylodromus affinis (GERHARDT, } \\
\text { 1877) }\end{array}$ & O & & $\begin{array}{l}\text { 9a(1), 22(7), 48(1), 54(1), 64(1), 68(1), 76(6), 82a(1), } \\
135(3)\end{array}$ & ATHW00 \\
\hline $\begin{array}{l}\text { Xylodromus depressus (GRAVEN- } \\
\text { HORST, 1802) }\end{array}$ & 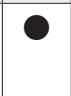 & & $54(1)$ & \\
\hline \multicolumn{5}{|l|}{ Proteininae } \\
\hline $\begin{array}{l}\text { Megarthrus depressus (PAYKULL, } \\
1789 \text { ) }\end{array}$ & 0 & $\odot$ & $63(1), 105(10)$ & \\
\hline $\begin{array}{l}\text { Megarthrus stercorarius MULSANT } \\
\text { \& ReY, } 1878\end{array}$ & $\bullet$ & $\odot$ & $11 \mathrm{~b}(1), 88(2), 63(6), 105(14)$ & \\
\hline Metopsia similis ZERCHE, 1998 & $\bullet$ & $\bullet$ & $35(8), 66(4), 98(1), 112(2), 113(1)$ & \\
\hline $\begin{array}{l}\text { Proteinus atomarius ERICHSON, } \\
1840\end{array}$ & 0 & • & $63(1), 69(1), 105(28)$ & \\
\hline $\begin{array}{l}\text { Proteinus baculatus Assing, } \\
\text { spec. nov. }\end{array}$ & $\bullet$ & & $8(1), 118(1)$ & \\
\hline $\begin{array}{l}\text { Proteinus brachypterus (FABRICIUS, } \\
\text { 1792) }\end{array}$ & & ○ & $114(1)$ & \\
\hline \multicolumn{5}{|l|}{ Micropeplinae } \\
\hline $\begin{array}{l}\text { Micropeplus marietti JACQUELIN } \\
\text { DU VAL, } 1857\end{array}$ & & 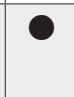 & $114(4), 116(1)$ & \\
\hline \multicolumn{5}{|l|}{ Dasycerinae } \\
\hline $\begin{array}{l}\text { Dasycerus crenatus MотSCHULSкY, } \\
1839\end{array}$ & 0 & & $70(1)$ & \\
\hline \multicolumn{5}{|l|}{ Pselaphinae } \\
\hline Batrisodes delaporti (AUBÉ, 1833) & $\bigcirc$ & & & E30 \\
\hline $\begin{array}{l}\text { Batrisodes ruprechtii (KolENATI, } \\
1846 \text { ) }\end{array}$ & $\bigcirc$ & 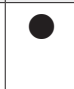 & $66 \mathrm{~b}(2), 98(3)$ & LB04 \\
\hline Bibloplectus pusillus (DeNNY, 1825) & 0 & & & LB04 \\
\hline
\end{tabular}




\begin{tabular}{|c|c|c|c|c|}
\hline Species & AR & NK & Localities/Samples & References \\
\hline $\begin{array}{l}\text { Bibloporus variicolor REITTER, } \\
1882\end{array}$ & 0 & & $53(1)$ & \\
\hline $\begin{array}{l}\text { Brachygluta araxidis (REITTER, } \\
\text { 1889) }\end{array}$ & O & & & SBBB04 \\
\hline $\begin{array}{l}\text { Brachygluta fossulata (REICHEN- } \\
\text { BACH, 1816) }\end{array}$ & O & & & LB04 \\
\hline $\begin{array}{l}\text { Brachygluta furcata (MoTsCHULSKY, } \\
\text { 1835) }\end{array}$ & $\bigcirc$ & & & SBBB04 \\
\hline $\begin{array}{l}\text { Brachygluta nodosa (MоT- } \\
\text { sCHULSKY, 1835) }\end{array}$ & O & ○ & $\begin{array}{l}36(22), 42(1), 66(4), 66 \mathrm{a}(4), 90(8), 90 \mathrm{a}(4), 103(22), \\
104(3), 109(36), 110(3), 114(1), 121 \mathrm{a}(5)\end{array}$ & SBBB04 \\
\hline $\begin{array}{l}\text { Brachygluta spinicoxis spinicoxis } \\
\text { (MoTSCHULSKY, 1835) }\end{array}$ & O & & & LB04 \\
\hline $\begin{array}{l}\text { Brachygluta sursicauda (GANGL- } \\
\text { BAUER, 1900) }\end{array}$ & 0 & & $\mathrm{~b}(1)$ & \\
\hline $\begin{array}{l}{ }^{* *} \text { Bryaxis armeniacus BRACHAT, } \\
\text { spec. nov. }\end{array}$ & 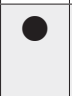 & & $4(21)$ & \\
\hline $\begin{array}{l}\text { Bryaxis bulbifer (REICHENBACH, } \\
\text { 1816) }\end{array}$ & O & 0 & $\begin{array}{l}6(4), 7(4), 9(23), 11(1), 11 b(1), 15(7), 19(8), 20(7), \\
22(30), 31(12), 33(3), 36(10), 39(31), 40(7), 48(4), \\
51(2), 52(1), 53(26), 55(15), 56(2), 57(4), 61(4), \\
62(11), 63(19), 66(5), 66 a(6), 66 b(9), 68(15), 68 a(4), \\
69(6), 70(4), 72(8), 75(1), 76(6), 81(4), 81 a(10), \\
82(2), 89(32), 89 b(45), 90(12), 97(2), 100(11), \\
101(10), 103(5), 105(17), 106(2), 107(2), 109(3), \\
110(1), 112(2), 117(9), 119(38), 121(1), 125(7), \\
126(27), 127(8), 130(6), 131(18), 199 a(7)\end{array}$ & BK07 \\
\hline $\begin{array}{l}\text { Bryaxis clavipes (MOTSCHULSKY, } \\
1851 \text { ) }\end{array}$ & $\bigcirc$ & $\bigcirc$ & $\begin{array}{l}\text { 5(1), 55(10), 63(1), 66a(22), 66b(12), 70(2), 72(2), } \\
81(5), 81 \mathrm{a}(1), 88(3), 98(6), 100(8), 101(7), 102(11), \\
105(25), 108(2), 109(4), 110(74), 112(2), 113(8), \\
114(6), 117(4)\end{array}$ & BK07 \\
\hline $\begin{array}{l}\text { Bryaxislongipalpis(MotschULSKY, } \\
\text { 1835) }\end{array}$ & O & & $5(1), 6(1), 51(1), 58(26), 66 b(3), 70(6), 72(1)$ & BK07 \\
\hline $\begin{array}{l}{ }^{* *} \text { Bryaxis meghruicus BRACHAT, } \\
\text { spec. nov. }\end{array}$ & 0 & & $120(1)$ & \\
\hline $\begin{array}{l}\text { Bryaxis rostratus (MOTSCHULSKY, } \\
1845 \text { ) }\end{array}$ & $\bigcirc$ & & & BK07 \\
\hline $\begin{array}{l}{ }^{* *} \text { Bryaxis seductus Besuchet \& } \\
\text { KuRBATOv, } 2007\end{array}$ & O & & $30(25), 31(1), 121(1)$ & BK07 \\
\hline $\begin{array}{l}\text { Bythinus gracilis MOTSCHULSKY, } \\
1851\end{array}$ & $\bigcirc$ & 0 & $\begin{array}{l}22(1), 33(13), 36(3), 39(8), 40(2), 50(2), 52(3), \\
53(3), 61(6), 63(14), 66 a(5), 66 b(6), 70(3), 81(1), \\
81 a(3), 88(21), 89(3), 89 b(1), 90(1), 103(1), 105(4), \\
109(2), 110(8), 199 a(7)\end{array}$ & LB04 \\
\hline $\begin{array}{l}\text { Chennium prometheus SAULCY, } \\
1875\end{array}$ & O & & & LB04 \\
\hline $\begin{array}{l}\text { Claviger colchicus Motschulsky, } \\
1837\end{array}$ & O & & & LB04 \\
\hline Ctenistes marthae ReITteR, 1891 & $\bigcirc$ & & & LB04 \\
\hline $\begin{array}{l}\text { Ctenistes palpalis REICHENBACH, } \\
1816\end{array}$ & O & & & LB04 \\
\hline $\begin{array}{l}\text { Enoptostomus globulicornis (MоT- } \\
\text { sCHULSKY, 1851) }\end{array}$ & $\bigcirc$ & & & E30 \\
\hline
\end{tabular}




\begin{tabular}{|c|c|c|c|c|}
\hline Species & AR & NK & Localities/Samples & References \\
\hline $\begin{array}{l}\text { Euplectus piceus piceus Мот- } \\
\text { sCHULSкY, } 1835\end{array}$ & $\bigcirc$ & & & LB04 \\
\hline $\begin{array}{l}\text { Plectophloeus nubigena nubigena } \\
\text { (ReItTER, 1877) }\end{array}$ & $\bullet$ & & $31(1), 70(1)$ & \\
\hline $\begin{array}{l}\text { Pselaphus acuminatus Мот- } \\
\text { sCHULSKY, } 1835\end{array}$ & 0 & & 121(3), 121a(9), 212(1) & LB04 \\
\hline Pselaphus caspicus Reitter, 1882 & $\bullet$ & $\bullet$ & 66(1), 66a(1), 98(1), 100(1) & \\
\hline $\begin{array}{l}\text { Pselaphus caucasicus Мот- } \\
\text { schulsкy, } 1845\end{array}$ & O & & & LB04 \\
\hline Rybaxis longicornis (LEACH, 1817) & $\bigcirc$ & $\bullet$ & 66(1), 66a(5), 100(1), 105(1) & LB04 \\
\hline $\begin{array}{l}\text { Trimium caucasicum KolENATI, } \\
1846\end{array}$ & $\bigcirc$ & & 53(2), 66(2), 66b(4), 70(2) & LB04 \\
\hline $\begin{array}{l}\text { Trissemus antennatus serricornis } \\
\text { SCHмIDT-GöBEL, } 1838\end{array}$ & $\bullet$ & & $66 \mathrm{a}(1)$ & \\
\hline Trissemus melinus (SoLSKY, 1869) & $\bigcirc$ & & $177(1)$ & LB04 \\
\hline $\begin{array}{l}\text { Trissemus montanus (SAULCY, } \\
\text { 1876) }\end{array}$ & O & & $130(1)$ & LB04 \\
\hline Tychus armeniacus SAULCY, 1878 & - & & $66(1)$ & \\
\hline Tychus colchicus SAULCY, 1878 & $\bigcirc$ & & $9(1), 19(2), 81 \mathrm{a}(1)$ & LB04 \\
\hline $\begin{array}{l}\text { Tychus dubius SABELLA \& KuRBA- } \\
\text { TOv, } 2002\end{array}$ & 0 & & $88(1)$ & \\
\hline Tychus guillebeaui RAFFRAY, 1904 & 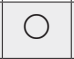 & & $54(1), 89(2)$ & LB04 \\
\hline Tychus lederi SAulcy, 1878 & $O$ & & & LB04 \\
\hline $\begin{array}{l}\text { **Tychus milvus SABELla \& } \\
\text { Kurbatov, } 2002\end{array}$ & O & & & SKu02 \\
\hline $\begin{array}{l}\text { Tychus remaudierei BESUCHET, } \\
1969\end{array}$ & $O$ & & & SKu02 \\
\hline *Zibus yunonae KaLASHIAN, 1990 & $\bigcirc$ & & & Kn90 \\
\hline \multicolumn{5}{|l|}{ Tachyporinae } \\
\hline $\begin{array}{l}\text { Bolitobius castaneus boreomontani- } \\
\text { cus SCHÜLKE, } 2010\end{array}$ & - & $\bullet$ & $82(1), 108(1), 112(1), 148 \mathrm{~d}(1), 148 \mathrm{f}(1), 152(1)$ & \\
\hline \begin{tabular}{|l}
$\begin{array}{l}\text { Bolitobius castaneus castaneus } \\
\text { (STEPHENS, 1832) }\end{array}$ \\
\end{tabular} & 0 & & 11(1), 11b(1), 47(5), 68(1) & \\
\hline $\begin{array}{l}\text { Bryophacis rugipennis (PANDELLÉ, } \\
\text { 1869) }\end{array}$ & 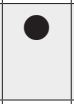 & & $o(1)$ & \\
\hline $\begin{array}{l}\text { Bryoporus cernuus (GRAVENHORST, } \\
\text { 1806) }\end{array}$ & 0 & & 59(1), 97(1), 150c(2), 152(1), p(1) & \\
\hline $\begin{array}{l}\text { Bryoporus multipunctus HAMPE, } \\
1867\end{array}$ & $\bullet$ & & $148 \mathrm{~d}(1), \mathrm{q}(1)$ & \\
\hline Cilea silphoides (LinnaEus, 1767) & $\bigcirc$ & & & Sm04 \\
\hline $\begin{array}{l}\text { Ischnosoma longicorne (MäKLIN, } \\
\text { 1847) }\end{array}$ & $O$ & $\bullet$ & $\begin{array}{l}1(1), 2(3), 3(5), 3 \mathrm{a}(1), 6(4), 7(5), 11(1), 22(6), 42(1), \\
47(2), 50(3), 51(2), 52(3), 53(1), 64(1), 66 \mathrm{~b}(1), \\
72(1), 75(8), 77(1), 88(10), 89(7), 90(1), 91(3), \\
98(6), 100(3), 110(4), 112(25), 114(1), 118(2), \\
119(1), 125(1), 127(18), 129(1), 131(1), 148 \mathrm{~b}(1), \\
148 \mathrm{~d}(1), 148 \mathrm{f}(2), 152(1), 154(1), 164(4), 167(1), \\
170(1), 199 \mathrm{a}(4)\end{array}$ & Sm04 \\
\hline
\end{tabular}




\begin{tabular}{|c|c|c|c|c|}
\hline Species & AR & NK & Localities/Samples & References \\
\hline $\begin{array}{l}\text { Ischnosoma splendidum (GRAVEN- } \\
\text { HORST, 1806) }\end{array}$ & O & 0 & $\begin{array}{l}\text { 1(3), 4(5),6(2),7(4), 9(9),19(2), 36(3), 39(4), 48(8), } \\
54(1), 67(1), 76(2), 78(3), 82(1), 89(1), 90 \mathrm{a}(2), \\
92(2), 103(1), 104(1), 117(1), 118(2), 119(1), 125(1), \\
126(22), 130(7), 150 \mathrm{~d}(1), 152(1), 165(1), 199 \mathrm{a}(4)\end{array}$ & \\
\hline $\begin{array}{l}\text { Lamprinodes haematopterus } \\
\text { (KRAATZ, 1857) }\end{array}$ & O & & $126(1)$ & \\
\hline $\begin{array}{l}\text { Lamprinus erythropterus (PANZER, } \\
1796 \text { ) }\end{array}$ & O & & & Sc08 \\
\hline $\begin{array}{l}\text { Lordithon bimaculatus (SCHRANK, } \\
\text { 1798) }\end{array}$ & 0 & & $60(1), r(1)$ & \\
\hline $\begin{array}{l}\text { Lordithon rostratus (MоT- } \\
\text { SCHULSKY, 1860) }\end{array}$ & 0 & & $\begin{array}{l}\text { 55(1), 57(29), 58(1), 60(!), 69(4), 135(1), 148d(1), } \\
\text { 153(1), s(1) }\end{array}$ & \\
\hline $\begin{array}{l}\text { Lordithon thoracicus thoracicus } \\
\text { (FABRICIUS, 1777) }\end{array}$ & ○ & - & $\begin{array}{l}4(7), 6(8), 7(3), 11(2), 11 \mathrm{a}(1), 11 \mathrm{~b}(3), 19(1), 30(1), \\
31(1), 36(2), 39(8), 50(2), 51(1), 53(22), 55(28), \\
57(6), 60(1), 61(2), 62(1), 63(2), 66 \mathrm{a}(2), 66 \mathrm{~b}(1), \\
67(1), 68(2), 68 \mathrm{a}(1), 69(3), 70(7), 74(1), 72(1), \\
76(3), 82(3), 89(4), 92(3), 100(2), 101 \mathrm{a}(1), 103(1), \\
105(1), 112(2), 116(1), 118(3), 118 \mathrm{a}(1), 119(10), \\
121 \mathrm{a}(2), 121 \mathrm{a}(2), 125(6), 126(36), 142(1), 143(1), \\
148 \mathrm{~b}(1), 148 \mathrm{~d}(2), 148 \mathrm{e}(1), 150(2), 153(1), 155(2), \\
206(1), \mathrm{t}(1)\end{array}$ & \\
\hline $\begin{array}{l}\text { Lordithon trimaculatus (FABRICIUS, } \\
\text { 1792) }\end{array}$ & 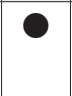 & & $136(4), \mathrm{u}(1)$ & \\
\hline $\begin{array}{l}\text { Mycetoporus bimaculatus LACOR- } \\
\text { DAIRE, } 1835\end{array}$ & O & & $20(1)$ & $\mathrm{Am} 00^{3)}, \mathrm{Sm} 04$ \\
\hline $\begin{array}{l}\text { Mycetoporus corpulentus LuzE, } \\
1901\end{array}$ & ○ & & 4(4), 7(1), 11(1), 52(1), 57(1), 90(1), 131(1) & \\
\hline $\begin{array}{l}\text { Mycetoporus eppelsheimianus } \\
\text { FAGEL, } 1968\end{array}$ & O & 0 & $\begin{array}{l}1(1), 11(4), 11 \mathrm{a}(4), 11 \mathrm{~b}(2), 12(11), 13(1), 76(2) \\
77(1), 81(2), 82(1), 92(1), 116(5), 118(1), 119(1)\end{array}$ & $\mathrm{Am00}{ }^{4)}$ \\
\hline $\begin{array}{l}\text { Mycetoporus forticornis FAUVEL, } \\
1875\end{array}$ & O & & $88(1), 172(1)$ & \\
\hline $\begin{array}{l}\text { Mycetoporus glaber glaber (SPERK, } \\
\text { 1835) }\end{array}$ & O & & $49 \mathrm{a}(1), 50(7), 52(1), 73(2), 76(4), 78(2), 151 \mathrm{c}(1)$ & SK00 \\
\hline $\begin{array}{l}\text { Mycetoporus imperialis BERN- } \\
\text { HAUER, } 1902\end{array}$ & O & O & $\begin{array}{l}32(1), 33(1), 39(1), 112(2), 118(1), 121(1), 126(6) \\
130(1), 148 d(1), w(1)\end{array}$ & \\
\hline $\begin{array}{l}\text { Mycetoporus lepidus (GRAVEN- } \\
\text { HORST, 1806) }\end{array}$ & O & & & $\mathrm{Sm} 04$ \\
\hline Mycetoporus liliputanus Luze, 1901 & ○ & 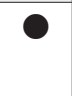 & $\begin{array}{l}26(1), 35(2), 77(1), 78(3), 79(51), 81(3), 81 \mathrm{a}(2) \\
98(1), 107(1), 110(1), 112(1), 114(1), 116(2)\end{array}$ & \\
\hline $\begin{array}{l}\text { Mycetoporus longulus MANNER- } \\
\text { HEIM, } 1830\end{array}$ & O & & 19(3) & Sc08 \\
\hline $\begin{array}{l}\text { Mycetoporus monticoloides } \\
\text { SCHÜLKE, } 2019\end{array}$ & O & 0 & 7(1), 63(2), 103(1), 118a(1), 121a(1) & Sc19a \\
\hline $\begin{array}{l}\text { Mycetoporus phaedrus (KolenATI, } \\
1846 \text { ) }\end{array}$ & O & O & $\begin{array}{l}4(1), 9(5), 10(2), 12(2), 13(16), 19(1), 22(4), 23(2), \\
30(1), 31(1), 32(1), 39(19), 40(1), 41(1), 47(1), \\
48(10), 49(2), 49 a(2), 50(4), 57(2), 70(3), 71(2), \\
74(5), 76(2), 77(1), 82(1), 83(3), 88(6), 89(10), \\
89 b(8), 101(1), 103(2), 107(2), 108(1), 110(3), \\
114(3), 116(26), 118(2), 118 a(1), 119(1), 126(16), \\
129(1)\end{array}$ & Ko46, Sc19a \\
\hline
\end{tabular}




\begin{tabular}{|c|c|c|c|c|}
\hline Species & AR & NK & Localities/Samples & References \\
\hline $\begin{array}{l}\text { Mycetoporus praetextoides } \\
\text { SCHÜLKE, } 2019\end{array}$ & 0 & ○ & $\begin{array}{l}4(1), 13(1), 32(66), 39(25), 74(2), 78(2), 79(2), \\
89 \mathrm{~b}(2), 103(3), 109(1), 110(1), 114(7), 118(1), \\
118 \mathrm{a}(2), 121(1), 126(11)\end{array}$ & Sc19a \\
\hline $\begin{array}{l}\text { Mycetoporus punctus (GRAVEN- } \\
\text { HORST, 1806) }\end{array}$ & O & & $\begin{array}{l}4(2), 11(1), 30(2), 48(1), 88(2), 126(1), 127(1) \\
159(1), x(1)\end{array}$ & $\mathrm{Sc} 08$ \\
\hline $\begin{array}{l}\text { Mycetoporus reichei (PANDELLÉ, } \\
\text { 1869) }\end{array}$ & O & ○ & $\begin{array}{l}3 \mathrm{a}(3), 11(8), 11 \mathrm{a}(1), 11 \mathrm{~b}(34), 12(11), 13(3), 15(4), \\
22(8), 22 \mathrm{a}(5), 26(2), 30(5), 31(3), 32(36), 33(7), \\
34(3), 39(8), 45(1), 50(7), 50 \mathrm{a}(1), 53(8), 66(1), \\
66 \mathrm{~b}(2), 67(1), 68(2), 70(2), 73(2), 75(2), 75(1), \\
76(2), 77(17), 78(8), 79(17), 81(1), 81 \mathrm{a}(1), 82 \mathrm{a}(2), \\
83(1), 88(5), 89(2), 89 \mathrm{~b}(7), 91(4), 95(4), 96(1), \\
97(3), 103(1), 105(2), 108(1), 110(1), 112(9), 113(1), \\
116(2), 121(1), 118(5), 118 \mathrm{a}(1), 119(1), 121(1), \\
124(2), 125(5), 126(6), 127(5), 129(8), 131(6), \\
146 \mathrm{a}(2), 164(4), 165(1), 167(2), 172(2), \mathrm{y}(1)\end{array}$ & Sm04 \\
\hline $\begin{array}{l}\text { Mycetoporus silvaticus IABLOKOFF- } \\
\text { KHNZORIAN, } 1962\end{array}$ & O & O & $\begin{array}{l}32(1), 42(1), 50(1), 66(1), 67(2), 74(1), 75(1), 76(3), \\
77(1), 89(3), 89 b(3), 96(1), 109(2), 110(1), 112(3), \\
113(2), 118 a(3), 119(2), 121(1), 122(1), 126(1), \\
129(1), 180(1), v(1)\end{array}$ & IK62 \\
\hline $\begin{array}{l}\text { Parabolitobius inclinans (GRAVEN- } \\
\text { HORST, 1806) }\end{array}$ & O & O & $114(1), 155(2)$ & Sm04 \\
\hline $\begin{array}{l}\text { Sepedophilus immaculatus } \\
\text { (STEPHENs, 1832) }\end{array}$ & 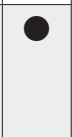 & O & $\begin{array}{l}\text { 6(1), 7(4), 11a(1), 60(1), 62(1), 66a(1), 68(1), 75(1), } \\
89(1), 91(1), 96(1), 98(1), 105(1), 113(1), 119(2), \\
127(3), 131(1), 150 \mathrm{~b}(2), 153(1), 163(2)\end{array}$ & \\
\hline Sepedophilus obtusus Luze, 1902 & 0 & 0 & $\begin{array}{l}1(1), 3 a(3), 6(3), 7(1), 11(3), 11 \mathrm{a}(5), 11 \mathrm{~b}(4), 13(1), \\
26(35), 32(8), 39(1), 40(1), 41(1), 47(1), 53(9), \\
57(1), 58(1), 66(1), 77(1), 79(1), 81(1), 88(15), \\
89(1), 91(1), 92(7), 93(1), 95(3), 96(6), 97(1), 98(5), \\
100(2), 101(2), 103(2), 104(1), 106(1), 112(2), \\
118(2), 118(6), 119(1), 121 \mathrm{a}(3), 125(1), 126(21), \\
129(2), 130(1), 131(1), 135(1), 138(1), 150(3), \\
150 c(2), 151 d(1), 162(2), 163(5), 164(8), 167(2), \\
168(3), 170(1), 180(3)\end{array}$ & \\
\hline $\begin{array}{l}\text { Sepedophilus rufulus (Носннштн, } \\
\text { 1849) }\end{array}$ & $\mathrm{O}$ & O & $\begin{array}{l}4(1), 9(1), 11 \mathrm{~b}(2), 20(1), 22(12), 22 \mathrm{a}(1), 30(1), \\
35(1), 39(1), 43(1), 50 \mathrm{a}(1), 64(3), 76(1), 78(1), \\
79(1), 80 \mathrm{~b}(1), 81 \mathrm{a}(1), 88(2), 112(2), 116(1), 118 \mathrm{a}(1), \\
121(1), 121 \mathrm{a}(1), 126(12), \mathrm{z}(1), \mathrm{a}(1)\end{array}$ & Sm04 \\
\hline $\begin{array}{l}\text { Sepedophilus testaceus (FABRICIUS, } \\
\text { 1792) }\end{array}$ & O & & $66 \mathrm{~b}(1), 68 \mathrm{a}(1)$ & E30 \\
\hline $\begin{array}{l}\text { Sepedophilus transcaucasicus } \\
\text { ScHüLKE, } 2019\end{array}$ & 0 & O & $\begin{array}{l}12(4), 26(1), 30(2), 32(10), 36(1), 53(2), 66(1), \\
78(2), 79(1), 88(2), 91(1), 92(3), 98(8), 100(1), \\
101(1), 103(3), 105(1), 109(1), 112(18), 113(1), \\
121(1), 126(15), 180(1), 196 a(1), 199 a(1)\end{array}$ & \\
\hline $\begin{array}{l}\text { Tachinus caucasicus Kolenati, } \\
1846\end{array}$ & O & O & $\begin{array}{l}\text { 135(1), 136(1), 148a(2), 148b(3), 148c(2), 154(1), } \\
\mathrm{ab}(1)\end{array}$ & Sm04, U75 ${ }^{64)}$ \\
\hline Tachinus cingulatus SoLSKY, 1864 & O & & & Sc89 \\
\hline $\begin{array}{l}\text { Tachinus corticinus GRAVENHORST, } \\
1802\end{array}$ & O & 0 & $\begin{array}{l}4(1), 6(1), 7(1), 16(1), 15(6), 19(5), 20(2), 22 \mathrm{a}(1), \\
41(5), 43(3), 49(7), 49 \mathrm{a}(1), 53(24), 54(2), 59(2), \\
70(2), 77(5), 78(2), 82(2), 82 \mathrm{a}(5), 89(1), 93(1), \\
98(1), 101(2), 103(2), 118(1), 121 \mathrm{a}(3), 142(1), \\
145(1), 160(1), 180(2)\end{array}$ & Am99a \\
\hline
\end{tabular}




\begin{tabular}{|c|c|c|c|c|}
\hline Species & AR & NK & Localities/Samples & References \\
\hline $\begin{array}{l}\text { Tachinus discoideus ERICHSON, } \\
1839\end{array}$ & O & & $204 \mathrm{a}(1)$ & Sm04 \\
\hline $\begin{array}{l}\text { Tachinus elongatus GYLLENHAL, } \\
1810\end{array}$ & O & & 16(1), 48(1), 49(1), 49a(1), 87(1) & U75 \\
\hline $\begin{array}{l}\text { Tachinus fimetarius GRAVEN- } \\
\text { HORST, } 1802\end{array}$ & O & & 30(2), 39(2), 41(5), 48(22), 50(1), 76(3), 82(1) & IK59 ${ }^{12)}$ \\
\hline $\begin{array}{l}\text { Tachinus humeralis marginicollis } \\
\text { KoLENATI, } 1846\end{array}$ & O & O & & $\mathrm{H}_{01}{ }^{5)}, \mathrm{Sc0} 4$ \\
\hline $\begin{array}{l}\text { Tachinus laticollis GRAVENHORST, } \\
1802\end{array}$ & O & 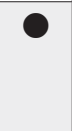 & $\begin{array}{l}4(2), 6(1), 7(3), 51(1), 53(3), 60(3), 62(1), 63(33) \\
68(2), 68 \mathrm{a}(4), 105(10), 148 \mathrm{~b}(2), 148 \mathrm{c}(2), 156(1) \\
181(1), 196 \mathrm{a}(1), 199 \mathrm{a}(1)\end{array}$ & $\mathrm{Sc} 04$ \\
\hline Tachinus nigerrimus SOLSKY, 1864 & $\bigcirc$ & & $44(7)$ & U75 \\
\hline $\begin{array}{l}\text { Tachinus pallipes GRAVENHORST, } \\
1806\end{array}$ & O & & & Am99a \\
\hline Tachinus rufipes (LinnaEus, 1758) & O & 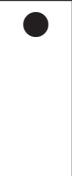 & $\begin{array}{l}1(2), 3(1), 4(1), 6(1), 11(1), 11 b(1), 19(1), 31(1), \\
39(1), 41(2), 45(3), 46(1), 49(3), 51(2), 63(2), \\
67(26), 68(2), 68 a(1), 69(3), 77(1), 81(1), 105(1), \\
123(1), 126(3), 128(5),\end{array}$ & Am99a \\
\hline $\begin{array}{l}\text { Tachinus rufitarsis Носннштн, } \\
1849\end{array}$ & O & & $46(1), 48(1), 76(2), 126(1)$ & Am99a \\
\hline Tachinus schneideri LuzE, 1900 & $\bigcirc$ & & & U75 \\
\hline $\begin{array}{l}\text { Tachinus subterraneus (LINNAEUS, } \\
\text { 1758) }\end{array}$ & O & & $\mathrm{ab}(2)$ & Am99a \\
\hline $\begin{array}{l}\text { Tachyporus atriceps STEPHENS, } \\
1832\end{array}$ & 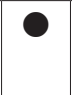 & 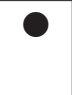 & $\begin{array}{l}\text { 13(1), 16(2), } 77(1), 78(5), 79(1), 88(2), 89 b(2) \\
98(5), 104(1), 167(1)\end{array}$ & \\
\hline $\begin{array}{l}\text { Tachyporus chrysomelinus } \\
\text { (LinnAEus, 1758) }\end{array}$ & O & & $\begin{array}{l}\text { 43(1), 47(1), 50(1), 64(10), 67(32), 76(3), 77(1), } \\
\text { 82(1), 82a(2), 87a(1), 88(1), 168(1), 182(1), 186(1), } \\
194(1)\end{array}$ & Sm04 \\
\hline Tachyporus dispar (PAYKULL, 1789) & 0 & & $13(1), 16(2), 165(1)$ & \\
\hline $\begin{array}{l}\text { Tachyporus fascipennis REITTER, } \\
1883\end{array}$ & 0 & & & ATHW00 \\
\hline $\begin{array}{l}\text { Tachyporus hypnorum (FABRICIUS, } \\
\text { 1775) }\end{array}$ & O & 0 & $\begin{array}{l}22(232), 22 \mathrm{a}(9), 23(1), 30(104), 31(43), 32(8), \\
33(1), 34(13), 36(7), 39(21), 40(1), 55(4), 57(10), \\
58(24), 60(1), 66(1), 66 a(1), 66 \mathrm{~b}(1), 70(14), 71(7), \\
72(3), 76(1), 78(106), 79(7), 81(24), 81 \mathrm{a}(13), 82(1), \\
88(186), 89(92), 89 \mathrm{~b}(173), 90(3), 90 \mathrm{a}(1), 91(16), \\
98(20), 101(1), 107(9), 108(29), 109(14), 110(43), \\
112(5), 113(7), 114(48), 115(6), 116(3), 117(2), \\
118 \mathrm{a}(1), 119(1), 121(2), 121 \mathrm{a}(1), 124(2), 125(4), \\
126(4), 127(16), 133(5), 135(1), 206(1), \mathrm{c}(1), \mathrm{d}(1)\end{array}$ & Ko46 ${ }^{58)}$ \\
\hline $\begin{array}{l}\text { Tachyporus nitidulus (FABRICIUS, } \\
\text { 1781) }\end{array}$ & 0 & 0 & $\begin{array}{l}3 \mathrm{a}(1), 4(17), 9(4), 11 \mathrm{~b}(1), 13(2), 15(3), 19(24), 20(3), \\
22(118), 22 \mathrm{a}(29), 30(13), 31(3), 32(1), 39(225), \\
40(9), 47(1), 48(1), 54(1), 55(1), 58(2), 59(4), 60(1), \\
61(1), 64(6), 69(1), 70(4), 71(4), 72(2), 76(6), 77(4), \\
78(17), 79(1), 81(5), 81 \mathrm{a}(14), 82(10), 82 \mathrm{a}(1), 88(52), \\
89(9), 89 \mathrm{a}(1), 89 \mathrm{~b}(15), 90(1), 90 \mathrm{a}(1), 91(1), 94(1), \\
98(7), 100(1), 101(1), 102(1), 103(1), 104(3), 105(1), \\
107(1), 108(7), 109(4), 110(10), 112(5), 113(6), \\
114(41), 115(5), 116(16), 117(2), 119(14), 121(13), \\
121 \mathrm{a}(4), 124(1), 125(1), 126(117), 127(2), 130(5), \\
131(5), 133(3), 134 \mathrm{a}(1), 199 \mathrm{a}(1), 203 \mathrm{a}(2), 207(3)\end{array}$ & ATHW00, E30 \\
\hline
\end{tabular}




\begin{tabular}{|c|c|c|c|c|}
\hline Species & AR & NK & Localities/Samples & References \\
\hline $\begin{array}{l}\text { Tachyporus obtusus (LINNAEUs, } \\
\text { 1767) }\end{array}$ & $\bigcirc$ & & & Sm04 \\
\hline $\begin{array}{l}\text { Tachyporus pusillus GRAVENHORsT, } \\
1806\end{array}$ & O & O & 36(36), 66(1), 81(2), 88(1), 98(1), 121a(8), 191a(1) & \\
\hline $\begin{array}{l}\text { Tachyporus scitulus ERICHSON, } \\
1839\end{array}$ & 0 & 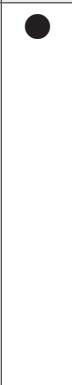 & $\begin{array}{l}9(11), 9 a(1), 12(1), 13(4), 15(1), 19(3), 21(1), \\
21 a(1), 22(1), 35(1), 36(1), 39(6), 41(4), 45(1), \\
47(3), 48(7), 50(47), 50 a(1), 53(5), 55(1), 64(37), \\
65(1), 66(2), 66 a(1), 73(2), 74(2), 75(4), 76(7), \\
78(1), 80(1), 81(87), 81 a(13), 82(2), 82 a(3), 83(3), \\
84(2), 85 a(30), 85 c(4), 87(1), 87 a(2), 88(15), 89(26), \\
89 b(40), 90(1), 91(2), 95(6), 103(1), 105(1), 121(1), \\
121 a(23), 126(23), 129(2), 130(5), 158(1), 160(2), \\
179(1), 204 a(1)\end{array}$ & \\
\hline $\begin{array}{l}\text { Tachyporus solutus ERICHSON, } \\
1839\end{array}$ & O & 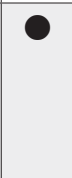 & $\begin{array}{l}13(2), 22 \mathrm{a}(1), 30(3), 33(1), 34(1), 36(1), 48(1), \\
50(2), 66(1), 66 \mathrm{~b}(1), 74(1), 75(1), 78(1), 81(1), \\
82(5), 88(2), 89(1), 92(1), 98(1), 104(1), 105(1), \\
112(3), 121(1), 126(3), 129(3), 135(1), 186(1)\end{array}$ & ATHW00 \\
\hline \multicolumn{5}{|l|}{ Habrocerinae } \\
\hline $\begin{array}{l}\text { Habrocerus capillaricornis } \\
\text { (GRAVENHORST, 1806) }\end{array}$ & & O & $98(5)$ & \\
\hline $\begin{array}{l}\text { Habrocerus simulans Assing \& } \\
\text { WUNDERLE, } 1995\end{array}$ & 0 & ○ & $\begin{array}{l}34(2), 35(2), 66(11), 66 a(5), 66 \mathrm{~b}(3), 88(11), 102(1), \\
112(11), 113(2), 121(2), 130(10), 163(1), 165(1), \\
167(3), 170(1), 199 \mathrm{a}(1)\end{array}$ & \\
\hline \multicolumn{5}{|l|}{ Ale ocharinae } \\
\hline Acrotona benicki (ALlen, 1940) & 0 & & $\begin{array}{l}\text { 66a(1), 74(2), 79(4), 80b(2), 81(10), 81a(27), 82(1), } \\
89(5), 89 \mathrm{~b}(3), 121 \mathrm{a}(1), 126(1)\end{array}$ & \\
\hline $\begin{array}{l}\text { Acrotona concamerata Assing, } \\
2004\end{array}$ & 0 & & $76(30), 77(1)$ & \\
\hline Acrotona exigua (ERICHSON, 1837) & O & & $19(22), 22(1)$ & \\
\hline $\begin{array}{l}\text { Acrotona muscorum (BRIsOUT, } \\
1860 \text { ) }\end{array}$ & $\bigcirc$ & 0 & $\begin{array}{l}13(1), 19(1), 22(5), 22 \mathrm{a}(2), 30(1), 31(2), 35(1), \\
36(1), 39(2), 50(1), 63(1), 70(1), 72(1), 80 \mathrm{~b}(3), \\
81(8), 81 \mathrm{a}(2), 88(2), 89 \mathrm{~b}(1), 92(1), 95(1), 97(3), \\
98(3), 102(6), 103(5), 105(3), 107(1), 108(2), \\
109(1), 110(28), 112(1), 113(1), 114(22), 115(1), \\
116(11), 118(1), 119(2), 121 \mathrm{a}(2), 125(1), 126(5), \\
127(3), 130(2)\end{array}$ & Be74 \\
\hline $\begin{array}{l}\text { Acrotona obfuscata (GRAVEN- } \\
\text { HORST, 1802) }\end{array}$ & O & ○ & $\begin{array}{l}\text { 5(1), 33(4), 39(6), 100(17), 126(22), 180(1), 199(2), } \\
199 \mathrm{a}(3)\end{array}$ & \\
\hline $\begin{array}{l}\text { Acrotona parvula (MANNERHEIM, } \\
\text { 1830) }\end{array}$ & 0 & & $44(10), 66(1)$ & \\
\hline $\begin{array}{l}\text { \#Aleochara armeniaca LikovskÝ, } \\
1971\end{array}$ & O & & & ATHW00, Li71 \\
\hline $\begin{array}{l}\text { Aleochara bilineata GYLLENHAL, } \\
1810\end{array}$ & $\bigcirc$ & & & ATHW00 \\
\hline $\begin{array}{l}\text { Aleochara bipustulata (LINNAEUs, } \\
1760 \text { ) }\end{array}$ & ○ & 0 & $\begin{array}{l}13(1), 18(1), 21(60), 21 \mathrm{a}(2), 24(1), 28 \mathrm{a}(1), 33(1) \\
77(1), 81(7), 81 \mathrm{a}(4), 82(1), 105(1), 106(1), 107(16), \\
109(1), 110(2), 113(1), 114(3), 120(1), 121(1), \\
122(7), 123(19), 124(1), 126(17), 212(1)\end{array}$ & \\
\hline $\begin{array}{l}\text { Aleochara brevipennis GrAVEN- } \\
\text { HORST, } 1806\end{array}$ & O & & $77(1)$ & A18a \\
\hline
\end{tabular}




\begin{tabular}{|c|c|c|c|c|}
\hline Species & AR & NK & Localities/Samples & References \\
\hline $\begin{array}{l}\text { Aleochara cephalotes BERNHAUER, } \\
1901\end{array}$ & $\bigcirc$ & & & Li71 \\
\hline $\begin{array}{l}\text { Aleochara conviva EPPELSHEIM, } \\
1878\end{array}$ & O & & $32(1), 165(5)$ & A18a \\
\hline $\begin{array}{l}\text { Aleochara cuniculorum KRAATZ, } \\
1858\end{array}$ & $\bigcirc$ & & & $\begin{array}{l}\text { ATHW00, } \\
\text { Li71 }^{51)}\end{array}$ \\
\hline Aleochara curtula (GoEzE, 1777) & O & & $121 \mathrm{a}(1), 151 \mathrm{~b}(1), 155(55)$ & \\
\hline $\begin{array}{l}\text { Aleochara erythroptera GRAVEN- } \\
\text { HORST, } 1806\end{array}$ & $\bigcirc$ & & $89 \mathrm{a}(1)$ & A18a \\
\hline Aleochara falcata Assing, 2009 & O & & $17(18), 87(2)$ & A18a \\
\hline $\begin{array}{l}\text { \#Aleochara fugax IABLOKOFF- } \\
\text { KHNZORIAN, } 1962\end{array}$ & $\bigcirc$ & & & $\begin{array}{l}\text { ATHW00, } \\
\text { IK62, IK64b, } \\
\text { Li71 10) }\end{array}$ \\
\hline $\begin{array}{l}\text { Aleochara grandeguttata Assing, } \\
2009\end{array}$ & O & & $7(3), 7 \mathrm{a}(1), 85 \mathrm{~b}(1), 187(2)$ & A09a, A18a \\
\hline $\begin{array}{l}\text { Aleochara haematoptera KRAATZ, } \\
1858\end{array}$ & O & & $8 \mathrm{~b}(2), 36 \mathrm{a}(1), 120(5), 189(2), 210(1)$ & $\begin{array}{l}\text { A17a, A18a, } \\
\text { Am00 }{ }^{8)}, \text { Li71 }^{9)}\end{array}$ \\
\hline Aleochara inconspicua AuBÉ, 1850 & O & O & $\begin{array}{l}22(28), 22 \mathrm{a}(2), 26(1), 30(1), 31(3), 36(1), 39(7), \\
41(2), 69(1), 70(3), 81(4), 81 \mathrm{a}(1), 88(10), 89(17), \\
89 \mathrm{~b}(23), 91(5), 110(3), 114(2), 116(4), 126(6), \\
165(1), 172(1)\end{array}$ & A18a \\
\hline $\begin{array}{l}\text { Aleochara intricata MANNERHEIM, } \\
1830\end{array}$ & O & & $4(1), 8(1), 148(1), 151 b(1)$ & SS15 \\
\hline $\begin{array}{l}\text { Aleochara lanuginosa GRAVEN- } \\
\text { HORST, } 1802\end{array}$ & O & ○ & $88(1), 115(5), 116(1)$ & A18a \\
\hline $\begin{array}{l}\text { Aleochara lata GRAVENHORST, } \\
1802\end{array}$ & O & & & IK64b \\
\hline Aleochara laticornis KRAATZ, 1856 & $\mathrm{O}$ & & $151 \mathrm{a}(1), 155(2), 157(1)$ & Li71 \\
\hline Aleochara maculata BRIsout, 1863 & O & & $16(1), 45(4), 58(1), 81 \mathrm{a}(1)$ & A18a \\
\hline $\begin{array}{l}\text { Aleochara maculipennis BAUDI DI } \\
\text { SELVE, } 1857\end{array}$ & $\bigcirc$ & & & Li71 \\
\hline Aleochara milleri KRAATZ, 1862 & $\mathrm{O}$ & & & E30 \\
\hline $\begin{array}{l}\text { Aleochara moerens GYLLENHAL, } \\
1827\end{array}$ & O & & 23(b), $148 b(1)$ & A18a \\
\hline $\begin{array}{l}\text { Aleochara moesta Gravenhorst, } \\
1802\end{array}$ & O & & & E30 \\
\hline $\begin{array}{l}\text { Aleochara morion (GRAVENHORST, } \\
1802 \text { ) }\end{array}$ & 0 & & $8(1)$ & \\
\hline Aleochara peeziana LOHSE, 1961 & $\bigcirc$ & & & Li71 \\
\hline Aleochara plicelytrata Assing, 2018 & O & - & $\begin{array}{l}20 \mathrm{a}(2), 22(1), 31(1), 39(12), 53(2), 57(2), 58(1) \\
59(2), 75(1), 89(12), 89 \mathrm{~b}(4), 90(1), 91(8), 100(16), \\
104(1), 105(4), 125(1), 126(10), 127(1), 156(2) \\
157(1)\end{array}$ & A18a \\
\hline $\begin{array}{l}{ }^{*} \text { Aleochara polychroma IABLO- } \\
\text { KOFF-KHNZORIAN, } 1966\end{array}$ & O & & & A17a, IK66 \\
\hline Aleochara sparsa HeER, 1839 & O & & $\begin{array}{l}54(1), 63(1), 75(1), 133(1), 135(23), 136(1), 145(17), \\
145 \mathrm{a}(36), 145 \mathrm{~b}(66), 146(39), 146 \mathrm{a}(186), 148(3), \\
148 \mathrm{~b}(17), \quad 148 \mathrm{c}(3), \quad 149(12), 149 \mathrm{a}(5), 149 \mathrm{~b}(9), \\
150(6), 151(11), 155(22), 156(18)\end{array}$ & ATHW00, Li71 \\
\hline
\end{tabular}




\begin{tabular}{|c|c|c|c|c|}
\hline Species & AR & NK & Localities/Samples & References \\
\hline $\begin{array}{l}\text { Aleochara subtumida (Носннuтн, } \\
\text { 1849) }\end{array}$ & $O$ & $\bullet$ & 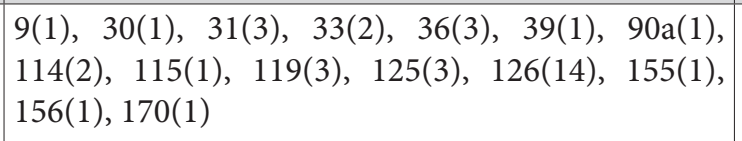 & $\begin{array}{l}\text { A18a, Am99b, } \\
\text { IK60 }\end{array}$ \\
\hline $\begin{array}{l}\text { Aleochara tristis (GRAVENHORST, } \\
\text { 1806) }\end{array}$ & 0 & & & Ep78 \\
\hline $\begin{array}{l}\text { Aleochara vagepunctata KRAATZ, } \\
1856\end{array}$ & 0 & & & ATHW00, Li71 \\
\hline Aleochara verna SAY, 1833 & $\bullet$ & & $44(35), 49(1)$ & \\
\hline $\begin{array}{l}\text { Aleochara villosa MANNERHEIM, } \\
1830\end{array}$ & 0 & & & Li71 \\
\hline $\begin{array}{l}\text { Alevonota gracilenta (ERICHSON, } \\
1839 \text { ) }\end{array}$ & 0 & & & AW08 \\
\hline $\begin{array}{l}\text { Alevonota hepatica (ERICHSON, } \\
1839)^{65}\end{array}$ & $\bullet$ & & $6(1), 68(1), 133(1), 135(1)$ & \\
\hline Alevonota libanotica (FAGEL, 1965) & $\bullet$ & & $4(1), 58(1)$ & \\
\hline $\begin{array}{l}\text { Aloconota cambrica (Wollaston, } \\
\text { 1855) }\end{array}$ & 0 & & 190(1), 199(1), 204a(1) & Be74 \\
\hline \#Aloconota differens (BENICK, 1974) & $O$ & & & Be74 \\
\hline $\begin{array}{l}\text { Aloconota gregaria (ERICHSON, } \\
\text { 1839) }\end{array}$ & 0 & & $67(1), 161(1)$ & Sm04 \\
\hline $\begin{array}{l}\text { Aloconota insecta (Tномsоn, } \\
1856 \text { ) }\end{array}$ & $\bullet$ & $\bullet$ & $\begin{array}{l}\text { 39(1), 100(1), 104(1), 112(1), 121a(2), 126(1), } \\
\text { 130(3), 199a(17) }\end{array}$ & \\
\hline $\begin{array}{l}\text { Aloconota mediterranea (Тном- } \\
\text { son, 1856) }\end{array}$ & $\bullet$ & & $67(1)$ & \\
\hline Amidobia talpa (HEer, 1841) & $\bullet$ & & $89 a(1)$ & \\
\hline $\begin{array}{l}\text { Amischa analis (GRAVENHORST, } \\
\text { 1802) }\end{array}$ & $\bullet$ & & $\begin{array}{l}\text { 5(1), 8a(1), 9(1), 16(1), 19(1), 20(10), 45(1), 46(1), } \\
49(3), 54(2), 60(1), 67(1), 203 a(1)\end{array}$ & \\
\hline $\begin{array}{l}\text { Amischa bifoveolata (MANNER- } \\
\text { HEIM, 1830) }\end{array}$ & $\bullet$ & & $\begin{array}{l}\text { 49(10), 49a(1), 54(3), 67(1), 73(1), 85a(1), 203a(1), } \\
204 a(1)\end{array}$ & \\
\hline Amischa decipiens (SHARP, 1869) & $\bullet$ & & 176(1), 201(1) & \\
\hline $\begin{array}{l}\text { Amischa forcipata Mulsant \& } \\
\text { REY, } 1873\end{array}$ & $\bullet$ & & 196(1) & \\
\hline $\begin{array}{l}\text { Anaulacaspis caucasica (FAGEL, } \\
\text { 1969) }\end{array}$ & 0 & & 121(1), 121a(55), 130(32), 212(5) & A16a \\
\hline $\begin{array}{l}\text { Anaulacaspisnigra(GRAVENHORST, } \\
\text { 1802) }\end{array}$ & $O$ & $\bullet$ & $36(4,66 \mathrm{a}(4), 99(1), 100(2), 103(2), 117(1)$ & A16a, E30 \\
\hline Anaulacaspis persica (FAGEL, 1969) & $\bigcirc$ & & & A16a \\
\hline $\begin{array}{l}{ }^{*} \text { Atheta abscisa Assing \& VogeL, } \\
2017\end{array}$ & 0 & $\bullet$ & $\begin{array}{l}\text { 9(8), 10(2), 11b(1), 19(2), 20(1), 20a(1), 39(36), } \\
\text { 67(1), 76(33), 77(6), 81(12), 81a(4), 83(1), 104(2), } \\
\text { 126(268), 131(1) }\end{array}$ & AV17 \\
\hline Atheta aeneicollis (SHARP, 1869) & & $\bullet$ & 114(1), 115(1) & \\
\hline Atheta aequicollis BENICK, 1978 & & $\bullet$ & 104(1) & \\
\hline Atheta albomontis Assing, 2009 & 0 & & $77(1)$ & \\
\hline Atheta araxis BENICK, 1943 & 0 & & $\begin{array}{l}\text { 5(2), 8(2), 9(3), 16(1), 18(3), 21(19), 21a(1), 32(1), } \\
39(3), 45(11), 49(2), 76(4), 85 \mathrm{c}(1), 127(1), 139(1)\end{array}$ & $\mathrm{AV} 17^{67)}, \mathrm{Be} 74^{67)}$ \\
\hline $\begin{array}{l}\text { Atheta atramentaria (GYLLENHAL, } \\
\text { 1810) }\end{array}$ & $\bullet$ & $\bullet$ & 63(1), 82(1), 116(3), 177(1) & \\
\hline
\end{tabular}




\begin{tabular}{|c|c|c|c|c|}
\hline Species & AR & NK & Localities/Samples & References \\
\hline Atheta atricolor (SHARP, 1869) & 0 & & $44(1)$ & \\
\hline Atheta benickiella BRUNDIN, 1948 & O & O & 11a(5), 55(2), 60(1), 63(34), 89(1), 96(4), 114(1) & \\
\hline $\begin{array}{l}\text { Atheta bispinosa Assing \& VogeL, } \\
2017\end{array}$ & $\bigcirc$ & O & $\begin{array}{l}1(1), 2(2), 3(1), 4(325), 5(33), 6(8), 7(20), 8(2), \\
11(4), 11 a(2), 11 b(18), 12(1), 16(1), 23(3), 28(1), \\
43(6), 51(19), 55(1), 57(3), 58(1), 63(10), 68 a(3), \\
69(7), 74(9), 75(3), 76(13), 82(4), 82 a(1), 83(1), \\
114(3), 118(3), 119(1), 125(4), 138(2), 165(4), \\
170(1), 172(1), 196 a(1), 199 a(5)\end{array}$ & AV17 \\
\hline $\begin{array}{l}\text { Atheta brevapicalis Assing \& } \\
\text { VoGEL, } 2017\end{array}$ & O & O & $\begin{array}{l}15(1), 39(3), 82(5), 76(1), 77(2), 81(1), 104(1), \\
105(1), 119(1), 126(1), 130(4)\end{array}$ & A18b, AV17 \\
\hline $\begin{array}{l}{ }^{*} \text { Atheta brevitheca Assing \& } \\
\text { VoGEL, } 2017\end{array}$ & O & & $\begin{array}{l}5(4), 9(12), 39(4), 47(2), 48(10), 50(1), 73(3), \\
74(24), 76(41), 82 \mathrm{a}(1), 83(1), 86(1), 93(13), 94 \mathrm{a}(1), \\
123(5), 126(5), 139(1), 165(1), 204 \mathrm{a}(1)\end{array}$ & AV17 \\
\hline $\begin{array}{l}\text { Atheta britanniae BERNHAUER \& } \\
\text { SCHEERPELTZ, } 1926\end{array}$ & 0 & & $57(1), 69(1), 70(2), 133(3)$ & \\
\hline $\begin{array}{l}\text { Atheta castanoptera (MANNER- } \\
\text { HEIM, 1830) }\end{array}$ & O & 0 & $\begin{array}{l}6(4), 53(3), 55(2), 57(10), 62(1), 63(4), 66 a(1), \\
69(15), 70(12), 92(1), 100(1), 102(1), 105(49), \\
106(2), 108(2), 112(4), 117(2), 118(1), 125(4), \\
135(7), 136(3), 144(3), 148(3), 148 b(4), 148 c(3), \\
149(3), 151(16), 170(8)\end{array}$ & Ep78 \\
\hline Atheta cauta (ERICHSON, 1837) & 0 & 0 & $44(12), 50(1), 81(1), 88(1), 108(1)$ & \\
\hline $\begin{array}{l}\text { Atheta chefsurica (EPPELSHEIM, } \\
1880 \text { ) }\end{array}$ & O & 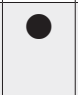 & $6(3), 60(1), 63(3), 105(15), 125(1)$ & A05a, IK60 \\
\hline $\begin{array}{l}\text { Atheta crassicornis (FABRICIUS, } \\
1792 \text { ) }\end{array}$ & 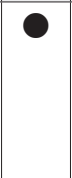 & 0 & $\begin{array}{l}\text { 6(2), 11b(2), 39(3), 48(1), 53(2), 55(2), 64(9), 72(2), } \\
98(1), 103(3), 118(1), 126(1), 135(1), 146(6), 148(2) \\
148 \mathrm{c}(7), 149(32), 149 \mathrm{a}(4), 149 \mathrm{~b}(4), 150 \mathrm{a}(1), 151(2) \\
155(4)\end{array}$ & \\
\hline Atheta dadopora Tномson, 1867 & O & & $6(1), 53(1), 63(2), 72(1), 135(2)$ & \\
\hline Atheta debilis (ERICHSON, 1837) & $\bigcirc$ & & $9(1)$ & E30 \\
\hline Atheta dissimulans Assing, 2007 & 0 & & $75(5)$ & \\
\hline $\begin{array}{l}\text { Atheta ebenina (MulsANT \& REY, } \\
\text { 1873) }\end{array}$ & ○ & 0 & $105(1), 126(1), 165(1)$ & \\
\hline Atheta epirotica BENICK, 1981 & 0 & & $76(1), 82(4), 82 \mathrm{a}(1)$ & \\
\hline \#Atheta erevanensis BENICK, 1985 & $\bigcirc$ & & & Be74, Be85 \\
\hline Atheta fimorum (BRISOUT, 1860) & $\bigcirc$ & & & $\mathrm{Be} 74$ \\
\hline Atheta fuscicolor BENICK, 1943 & O & O & $114(1), 126(1)$ & \\
\hline $\begin{array}{l}\text { Atheta gagatina (BAUDI DI SELVE, } \\
\text { 1848) }\end{array}$ & 0 & 0 & 6(10), 98(1), 100(3) & \\
\hline $\begin{array}{l}{ }^{*} \text { Atheta hamulata Assing \& } \\
\text { VogEL, } 2017\end{array}$ & O & & $\begin{array}{l}9(39), 9 a(6), 20 a(1), 33(1), 39(2), 48(6), 67(2), \\
76(36), 77(4), 82(16), 82 a(1), 93(2), 126(2)\end{array}$ & AV17 \\
\hline Atheta hansseni STRAND, 1943 & O & & $11 \mathrm{a}(1), 11 \mathrm{~b}(1)$ & AV17 \\
\hline Atheta harwoodi Williams, 1930 & O & & $135(1)$ & \\
\hline Atheta heymesi HubenthaL, 1913 & O & O & $89(1), 89 b(1), 105(2), 118(6), 118 a(2), 125(1)$ & \\
\hline Atheta hybrida SHARP, 1869 & 0 & & $135(32)$ & \\
\hline Atheta indubia (SHARP, 1869) & O & & $66 a(1)$ & \\
\hline Atheta ischnocera Thomson, 1870 & O & & $44(1)$ & \\
\hline Atheta judith (SAuLCY, 1865) & $\bigcirc$ & & $39(6), 126(5), 131(1), 162(7), 165(1), 167(5)$ & Be74 \\
\hline
\end{tabular}




\begin{tabular}{|c|c|c|c|c|}
\hline Species & AR & NK & Localities/Samples & References \\
\hline Atheta knabli BENICK, 1938 & 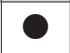 & & 9(7), 39(1), 42(3), 48(2), 126(2) & \\
\hline $\begin{array}{l}\text { Atheta laevana (MULSANT \& REY, } \\
\text { 1852) }\end{array}$ & $\bullet$ & $\bullet$ & 103(4), 110(1), 126(1) & \\
\hline Atheta laevicauda SAHLBERG, 1867 & $\bullet$ & $\bullet$ & $\begin{array}{l}4(3), 9(6), 10(1), 11(1), 13(2), 15(6), 20 \mathrm{a}(1), \\
21 \mathrm{a}(10), 22(1), 30(1), 31(1), 39(1), 43(1), 47(2), \\
48(1), 49(3), 50(4), 52(1), 74(2), 75(3), 76(2), 77(1), \\
78(1), 81 \mathrm{a}(1), 82 \mathrm{a}(1), 83(1), 88(3), 89(3), 89 \mathrm{~b}(14), \\
90(1), 90 \mathrm{a}(1), 91(4), 103(2), 105(1), 116(1), 118(9), \\
118 \mathrm{a}(2), 119(3), 126(4), 127(2), 129(2), 131(3), \\
183(1)\end{array}$ & \\
\hline $\begin{array}{l}\text { Atheta laevigata (НосннUтн, } \\
\text { 1849) }\end{array}$ & $\bullet$ & $\bullet$ & $\begin{array}{l}32(4), 49(16), 60(1), 73(2), 76(2), 77(1), 82(1), \\
85 c(1), 118(2), 125(3), 128(2), 181(1), 184(1), \\
203 \mathrm{a}(1)\end{array}$ & \\
\hline Atheta laticollis (STEPHENS, 1832) & O & $\bullet$ & $36(1), 110(1)$ & E30 \\
\hline Atheta liturata (STEPHENS, 1832) & $\bullet$ & & 69(93), 70(1) & \\
\hline $\begin{array}{l}\text { Atheta longicornis (GRAVENHORST, } \\
\text { 1802) }\end{array}$ & $\bullet$ & ○ & $\begin{array}{l}22(3), 30(1), 39(1), 44(1), 70(1), 80 \mathrm{~b}(4), 101(1), \\
107(1), 109(3), 113(1), 114(4), 116(1), 119(1), \\
121 \mathrm{a}(1), 126(19)\end{array}$ & \\
\hline $\begin{array}{l}\text { Atheta luctuosa (MULSANT \& REY, } \\
\text { 1853) }\end{array}$ & $\bullet$ & $\bullet$ & $32(1), 39(2), 40(1), 114(7), 121(2), 126(2)$ & \\
\hline Atheta lyciana Assing, 2003 & $\bullet$ & & $\begin{array}{l}\text { 11(1), 46(1), 49(3), 76(34), 77(2), 82(2), 83(1), } \\
85 c(3), 93(1), 128(54), 165(1)\end{array}$ & \\
\hline Atheta malleiformis BENICK, 1975 & & $\bullet$ & $114(2)$ & \\
\hline $\begin{array}{l}\text { **Atheta meghruica Assing, } \\
\text { spec. nov. }\end{array}$ & $\bullet$ & & $123(1), 126(2)$ & ASpp \\
\hline $\begin{array}{l}\text { Atheta melanaria (MANNERHEIM, } \\
\text { 1830) }\end{array}$ & $\bullet$ & O & $89 \mathrm{~b}(1), 109(1), 116(1)$ & Ko46 ${ }^{35)}$ \\
\hline $\begin{array}{l}\text { Atheta melanocera (THOMson, } \\
\text { 1856) }\end{array}$ & $\bullet$ & & 16(1), 21(10), 85c(13) & \\
\hline Atheta membranata BENICK, 1974 & $\bullet$ & & $53(1)$ & \\
\hline Atheta monstruosa Assing, 2011 & $\bullet$ & & 9(7), 10(1), 73(3), 76(4), 82(1), 127(1) & \\
\hline Atheta nigra (KRAATZ, 1856) & $\bullet$ & & $80 \mathrm{~b}(1), 82(1), 126(1)$ & \\
\hline $\begin{array}{l}\text { Atheta nigritula (GRAVENHORST, } \\
\text { 1802) }\end{array}$ & $\bullet$ & & $6(2), 11(2), 57(3), 148 \mathrm{~b}(9)$ & \\
\hline Atheta occulta (ERICHSON, 1837) & O & & & Be74 \\
\hline $\begin{array}{l}\text { Atheta pechlaneri SCHEERPELTZ, } \\
1933\end{array}$ & 0 & & $21 \mathrm{a}(2), 67(2), 86(6), 125(1)$ & AV17 \\
\hline Atheta putrida (KRAATZ, 1856) & $\bullet$ & & $39(1), 118(1)$ & \\
\hline Atheta ravilla (ERICHSON, 1839) & $\bullet$ & & $53(1), 60(8), 63(12), 64(2)$ & \\
\hline Atheta scabens Assing, 2011 & $\bullet$ & & $45(1)$ & \\
\hline $\begin{array}{l}\text { Atheta schneideri (EPPELSHEIM, } \\
\text { 1889) }\end{array}$ & 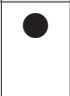 & & $\begin{array}{l}\text { 21(1), 39(3), 45(2), 73(12), 74(3), 81(2), 81a(1), } \\
118(3), 122(7), 123(3), 126(5)\end{array}$ & \\
\hline $\begin{array}{l}{ }^{*} \text { Atheta semialba Assing \& VOGEL, } \\
2017\end{array}$ & O & & $\begin{array}{l}\text { 5(1), 8(6), 20a(1), 21(8), 28a(1), 39(3), 45(9), 83(5), } \\
94(1), 123(1), 126(1), 145 \mathrm{~b}(1), 155(5)\end{array}$ & AV17 \\
\hline $\begin{array}{l}{ }^{*} \text { Atheta senticollis Assing \& } \\
\text { VogEL, } 2017\end{array}$ & O & & $63(2), 144(3)$ & AV17 \\
\hline Atheta serrata BENICK, 1938 & $\bullet$ & & $118(1)$ & \\
\hline Atheta singularis BERNHAUER, 1912 & O & & & Be74 \\
\hline
\end{tabular}




\begin{tabular}{|c|c|c|c|c|}
\hline Species & AR & NK & Localities/Samples & References \\
\hline Atheta sodalis (ERICHSON, 1837) & 0 & & $\begin{array}{l}\text { 6(1), 39(2), 75(1), 118(1), 121(8), 121a(2), 125(8), } \\
126(1), 127(2), 153(1)\end{array}$ & \\
\hline Atheta subglabra (SHARP, 1869) & 0 & & $127(1)$ & \\
\hline $\begin{array}{l}\text { Atheta subsinuata (ERICHSON, } \\
\text { 1839) }\end{array}$ & 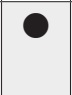 & & $44(8)$ & \\
\hline Atheta taxiceroides Munster, 1932 & 0 & & 53(1), 72(1), 88(1) & \\
\hline Atheta testaceipes (HEER, 1839) & O & & $88(1)$ & \\
\hline Atheta tibialis (HeER, 1839) & O & & $\begin{array}{l}\text { 16(1), 45(4), 46(10), 49(22), 67(29), 85a(5), 85b(1), } \\
94(1)\end{array}$ & Be74 \\
\hline Atheta triangulum (KRAATZ, 1856) & $\bigcirc$ & & $39(1), 81(1)$ & $\mathrm{Be} 74$ \\
\hline Atheta trinotata (KRAATZ, 1856) & 0 & & $86(1)$ & \\
\hline Atheta volans (SCRIBA, 1859) & O & & 19(1), 20(1), 94(1) & \\
\hline $\begin{array}{l}\text { Atheta xanthopus (Thomson, } \\
\text { 1856) }\end{array}$ & O & & $118(1)$ & \\
\hline $\begin{array}{l}\text { Autalia rivularis (GRAVENHORST, } \\
1802 \text { ) }\end{array}$ & O & O & $44(1), 105(1)$ & \\
\hline $\begin{array}{l}{ }^{* *} \text { Bellatheta khustupica Assing, } \\
\text { spec. nov. }\end{array}$ & O & & $122(6)$ & \\
\hline Bellatheta palata (BENICK, 1970) & O & & $50(5)$ & \\
\hline Bolitochara bella MäRKEL, 1844 & O & O & $98(3), 112(3), 134(1), 181(1)$ & \\
\hline $\begin{array}{l}\text { Bolitochara obliqua ERICHSON, } \\
1837\end{array}$ & O & 0 & $\begin{array}{l}\text { 1(1), 22(2), 39(1), 53(1), 68a(1), 71(2), 75(1), 92(1), } \\
\begin{array}{l}110(2), 112(1), 119(1), 126(5), 127(1), 135(2), \\
136(9), 138(1), 143(2), 144(2), 148(2), 150(1), \\
155(1), 156(2)\end{array}\end{array}$ & ATHW00 \\
\hline Bolitochara tecta Assing, 2014 & O & & $68 \mathrm{a}(2)$ & A14 \\
\hline Boreophilia eremita (RyE, 1866) & O & & $7(1), 19(1), 20(1), 21(30), 67(6), 85 c(8)$ & \\
\hline Brachida exigua (HEer, 1839) & O & O & $66(5), 79(3), 103(4)$ & \\
\hline $\begin{array}{l}\text { Brachyusa concolor (ERICHSON, } \\
1839 \text { ) }\end{array}$ & - & & $a(1)$ & \\
\hline $\begin{array}{l}{ }^{\star} \text { Calodera alticola Assing, } \\
\text { spec. nov. }\end{array}$ & 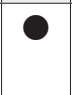 & & $128(1)$ & \\
\hline $\begin{array}{l}\text { Cordalia obscura (GRAVENHORST, } \\
\text { 1902) }\end{array}$ & 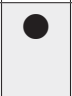 & & $36(1), 199(1), 199 a(15)$ & \\
\hline Cousya araxis (BERNHAUER, 1902) & $\bigcirc$ & O & $50(4), 70(1), 88(1), 107(1)$ & A18e \\
\hline $\begin{array}{l}\text { Cousya nigrata (FAIRMAIRE \& } \\
\text { LABOULBÈNE, 1856) }\end{array}$ & & O & $114(1), 116(1)$ & \\
\hline Cousya schuelkei Assing, 2007 & $\bigcirc$ & & $5(1), 48(5), 49 a(5), 122(1)$ & A18e \\
\hline $\begin{array}{l}\text { Cypha laeviuscula (MANNERHEIM, } \\
\text { 1830) }\end{array}$ & 0 & 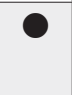 & 81a(2), 95(1), 103(2), 118(1) & \\
\hline Cypha spathulatha Assing, 2007 & O & O & $3(1), 98(1)$ & \\
\hline $\begin{array}{l}\text { Cypha takhtajani (IABLOKOFF- } \\
\text { KHNZORIAN, 1961) }\end{array}$ & O & & & A05a, IK61 \\
\hline Cypha tarsalis (LuzE, 1902) & ○ & O & $\begin{array}{l}11(2), 13(6), 33(1), 40(1), 76(1), 83(1), 98(1), \\
105(1), 119(3), 121 \mathrm{a}(1), 131(1), 165(7)\end{array}$ & \\
\hline Dinaraea aequata ERICHsON, 1837 & ○ & & $134(1)$ & \\
\hline $\begin{array}{l}\text { Dinaraea linearis (GRAVENHORST, } \\
1802 \text { ) }\end{array}$ & 0 & & $68 \mathrm{a}(2)$ & \\
\hline
\end{tabular}




\begin{tabular}{|c|c|c|c|c|}
\hline Species & AR & NK & Localities/Samples & References \\
\hline Dinarda hagensii WASMANN, 1889 & O & & $89 \mathrm{a}(6)$ & \\
\hline $\begin{array}{l}\text { Drusilla canaliculata (FABRICIUS, } \\
\text { 1787) }\end{array}$ & O & O & $\begin{array}{l}15(4), 19(29), 20(3), 20 \mathrm{a}(1), 30(1), 32(3), 33(1), \\
36(1), 40(2), 53(1), 64(10), 66(2), 73 \mathrm{a}(1), 78(7), \\
79(3), 80 \mathrm{a}(1), 88(10), 90(1), 101(2), 104(2), 109(1), \\
113(1), 114(2), 117(2), 126(9), 129(3), 130(2), \\
131(1), 148 \mathrm{~b}(7), 150(1), 151 \mathrm{a}(2), 151 \mathrm{~b}(3), 165(3), \\
167(1), 172(1), 183(2), 195(1)\end{array}$ & Ca39 ${ }^{38)}$ \\
\hline $\begin{array}{l}\text { Encephalus complicans STEPHENS, } \\
1832\end{array}$ & O & 0 & $7(1), 105(1)$ & \\
\hline Falagria caesa ERICHSON, 1837 & $\bigcirc$ & & $53(2), 58(1), 63(1), 177(1), 192(1)$ & Hh49 \\
\hline $\begin{array}{l}\text { Falagria sulcatula (GRAVENHORST, } \\
\text { 1806) }\end{array}$ & O & & $58(1), 150(1), 203 a(10)$ & E30 \\
\hline $\begin{array}{l}\text { Falagrioma thoracica (STEPHENS, } \\
\text { 1832) }\end{array}$ & 0 & 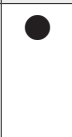 & $\begin{array}{l}33(1), 34(1), 35(8), 36(5), 39(1), 103(1), 112(9), \\
121(9), 121 \mathrm{a}(10), 126(8), 127(38), 130(22), 150 \mathrm{~b}(2), \\
152(1)\end{array}$ & \\
\hline${ }^{* *}$ Geostiba deliqua Assing, 2018 & O & & $118(3), 118 \mathrm{a}(1)$ & A18h \\
\hline${ }^{* * G e o s t i b a ~ i m m u t a t a ~ A s s i n g, ~} 2017$ & O & & $89(10), 89 b(8)$ & $\mathrm{A} 17 \mathrm{~b}$ \\
\hline $\begin{array}{l}{ }^{* *} \text { Geostiba kalavanica Assing, } \\
2017\end{array}$ & O & & $53(13)$ & A17b \\
\hline${ }^{* *}$ Geostiba khnzoriani PACE, 1983 & O & & 22(1), 22a(3), 34(3), 78(1), 88(7), 121a(1), 129(2) & $\begin{array}{l}\text { A17b, A18h, } \\
\text { Pa83 }\end{array}$ \\
\hline $\begin{array}{l}{ }^{* *} \text { Geostiba meghruica Assing, } \\
2016\end{array}$ & O & & $30(12), 31(1), 124(2)$ & A16b, A18h \\
\hline $\begin{array}{l}{ }^{* *} \text { Geostiba nigrohortensia Assing, } \\
2018\end{array}$ & & O & 105(96), 110(68), 113(1) & A18h \\
\hline $\begin{array}{l}{ }^{* *} \text { Geostiba pambakica Assing, } \\
2016\end{array}$ & O & & $5(10), 6(8), 8(3), 58(6), 70(39)$ & A16b, A17b \\
\hline Geostiba sororcula Assing, 2001 & O & & $\begin{array}{l}\text { 1(2), 3a(14), 9(1), 19(68), 23(1), 41(25), 42(24), } \\
43(1), 47(1), 48(22), 54(19), 76(1), 77(10), 80(1), \\
81 \mathrm{a}(18), 82(17), 121 \mathrm{a}(2)\end{array}$ & A17b, A18h \\
\hline${ }^{* *}$ Geostiba tigrani Assing, 2017 & O & & 50(50), 51(32), 52(10), 57(3), 68(15), 69(16) & A17b \\
\hline $\begin{array}{l}{ }^{* *} \text { Geostiba unicuneata Assing, } \\
2016\end{array}$ & O & O & 11(5), 11b(1), 81(1), 81a(2), 88(32), 105(16), 119(9) & $\begin{array}{l}\text { A16b, A17b, } \\
\text { A18h }\end{array}$ \\
\hline${ }^{* *}$ Geostiba unituber Assing, 2016 & O & & $4(23), 57(6), 62(1), 63(15)$ & A16b, A17b \\
\hline $\begin{array}{l}\text { Gnypeta carbonaria (MANNER- } \\
\text { HEIM,1830) }\end{array}$ & O & & & Sm04 \\
\hline \#Gnypeta gracilior BENICK, 1974 & O & & & Be74 \\
\hline Gnypeta rubrior ToтtenHaм, 1939 & 0 & & $7 \mathrm{a}(1), 187(1), 190(1)$ & \\
\hline $\begin{array}{l}\text { Gyrophaena affinis MANNERHEIM, } \\
1830\end{array}$ & ○ & O & $\begin{array}{l}\text { 10(4), 11(5), 11a(10), 11b(136), 12(1), 22(23), 39(8), } \\
53(7), 55(43), 62(1), 66(5), 66 \mathrm{a}(2), 69(8), 72(10), \\
75(2), 92(6), 101(1), 101 \mathrm{a}(1), 103(1), 105(177), \\
106(1), 108(1), 109(1), 110(3), 113(1), 119(7), \\
125(2), 126(2), 127(1)\end{array}$ & \\
\hline $\begin{array}{l}\text { Gyrophaena bihamata Thomson, } \\
1867\end{array}$ & 0 & O & $\begin{array}{l}\text { 1(3), 4(2), 33(2), 51(2), 55(1), 58(3), 62(4), 66(3), } \\
66 \mathrm{a}(2), 66 \mathrm{~b}(6), 70(2), 98(2), 100(1), 191 \mathrm{a}(2), 100(1), \\
103(1), 105(113), 106(1), 109(1), 110(3), 112(1), \\
113(1), 117(2), 126(3)\end{array}$ & \\
\hline $\begin{array}{l}\text { Gyrophaena boleti (LINNAEUs, } \\
1758 \text { ) }\end{array}$ & O & & & E30 \\
\hline
\end{tabular}




\begin{tabular}{|c|c|c|c|c|}
\hline Species & AR & NK & Localities/Samples & References \\
\hline $\begin{array}{l}\text { Gyrophaena caucasica STRAND, } \\
1939\end{array}$ & O & & 6(44), 34(1), 58(1), 66a(2), 142(2) & ES16 \\
\hline $\begin{array}{l}\text { Gyrophaena gentilis ERICHSON, } \\
1839\end{array}$ & O & 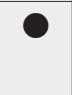 & $36(1), 50(1), 55(4), 60(8), 63(1), 105(3)$ & ES16 \\
\hline Gyrophaena hanseni STRAND, 1946 & 0 & & $70(5)$ & \\
\hline Gyrophaena joyi WENDELER, 1924 & O & O & $62(1), 101(1), 102(1), 105(16)$ & ES16 \\
\hline $\begin{array}{l}\text { Gyrophaena joyioides WüsTHOFF, } \\
1937\end{array}$ & ○ & & $66 a(3)$ & \\
\hline Gyrophaena korbi STRAND, 1939 & & 0 & $98(1), 101 \mathrm{a}(2), 102(1)$ & \\
\hline $\begin{array}{l}\text { Gyrophaena manca ERICHSON, } \\
1839\end{array}$ & 0 & & $66 \mathrm{a}(1), 68 \mathrm{a}(6), 69(7)$ & \\
\hline Gyrophaena nana (PAYKULL, 1800) & 0 & 0 & 55(17), 66(1), 70(1), 97(4), 105(6), 136(1) & \\
\hline $\begin{array}{l}\text { Gyrophaena orientalis STRAND, } \\
1938\end{array}$ & 0 & & $6(2)$ & \\
\hline $\begin{array}{l}\text { Haploglossa gentilis (MÄRKEL, } \\
\text { 1844) }\end{array}$ & 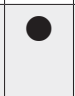 & & $148(1), 149 \mathrm{a}(2)$ & \\
\hline $\begin{array}{l}\text { Haploglossa picipennis (GYLLEN- } \\
\text { HAL, 1827) }\end{array}$ & $\bigcirc$ & & & $\begin{array}{l}\text { ATHW00, } \\
\text { IK64b }\end{array}$ \\
\hline $\begin{array}{l}\text { Haploglossa villosula (STEPHENS, } \\
\text { 1832) }\end{array}$ & O & & $135(3)$ & $\begin{array}{l}\text { ATHW00, } \\
\text { IK57a, IK64b }\end{array}$ \\
\hline $\begin{array}{l}\text { \#Hydrosmecta salsolae BENICK, } \\
1974\end{array}$ & O & & & $\mathrm{Be} 74$ \\
\hline $\begin{array}{l}\text { Ischnoglossa obscura WUNDERLE, } \\
1990\end{array}$ & 0 & & $59(1)$ & \\
\hline $\begin{array}{l}\text { Ischnopoda umbratica (ERICHSON, } \\
\text { 1837) }\end{array}$ & 0 & & 190(1), 196(3), 199(4), 199a(1), 206(1) & \\
\hline${ }^{* * 52)}$ Leptusa armeniaca PACE, 1989 & O & & $\begin{array}{l}22(41), 30(113), 31(50), 32(14), 33(24), 35(11), \\
40(3), 85 \mathrm{a}(1), 118(5), 124(21), 125(8), 127(2)\end{array}$ & A17c, Pa89 \\
\hline Leptusa fuliginosa (AuBÉ, 1850) & O & O & 60(1), 68(1), 68a(1), 69(1), 98(1) & A17c, Pa89 \\
\hline $\begin{array}{l}\text { Leptusa laeviuscula (Носннштн, } \\
\text { 1849) }\end{array}$ & O & & & Pa89 \\
\hline $\begin{array}{l}\text { Leptusa venusta (Носннштн, } \\
1849 \text { ) }\end{array}$ & O & & $\begin{array}{l}\text { 6(2), 7(4), 52(1), 66b(2), 68a(1), 72(2), 135(1), } \\
144(1), 148 \mathrm{~b}(1)\end{array}$ & A17c, Sz66a \\
\hline $\begin{array}{l}{ }^{\star} \text { Liogluta armeniaca Assing \& } \\
\text { VogEL, } 2017\end{array}$ & $\bigcirc$ & O & $\begin{array}{l}9(1), 22(5), 23(10), 28(9), 28 a(1), 31(1), 32(1), \\
33(1), 39(12), 40(1), 46(7), 48(2), 49(1), 67(17), \\
68(5), 69(17), 70(1), 71(3), 73(3), 74(9), 81(1), \\
83(1), 110(2), 113(1), 114(1), 118(1), 119(4), 126(4)\end{array}$ & AV17 \\
\hline $\begin{array}{l}\text { Liogluta granigera (KIESENWET- } \\
\text { TER, 1859) }\end{array}$ & O & & $\begin{array}{l}\text { 48(2), 51(1), 53(4), 55(58), 58(9), 60(13), 63(10), } \\
\text { 68(8), 60(19), 70(3), 71(1), 72(1), 135(5), 136(1), } \\
148(5), 148 \mathrm{c}(5), 149 \mathrm{a}(4), 199 \mathrm{a}(2)\end{array}$ & AV17, Be74 \\
\hline $\begin{array}{l}\text { Liogluta longiuscula (GRAVEN- } \\
\text { HORST, 1802) }\end{array}$ & 0 & 0 & $\begin{array}{l}\text { 57(3), 58(1), 90a(1), 106(1), 110(1), 126(1), 133(2), } \\
148(1), 149(1)\end{array}$ & \\
\hline $\begin{array}{l}\text { Liogluta microptera THомson, } \\
1867\end{array}$ & 0 & 0 & $\begin{array}{l}\text { 4(1), 5(1), 6(1), 7(1), 48(2), 61(1), 62(1), 91(1), } \\
\text { 105(1) }\end{array}$ & \\
\hline $\begin{array}{l}\text { Liogluta roettgeni (BERNHAUER, } \\
\text { 1903) }\end{array}$ & 0 & & & Be74 \\
\hline $\begin{array}{l}\text { Lomechusa paradoxa GRAVEN- } \\
\text { HORST, } 1806\end{array}$ & $\bigcirc$ & & & ATHW00 \\
\hline
\end{tabular}




\begin{tabular}{|c|c|c|c|c|}
\hline Species & AR & NK & Localities/Samples & References \\
\hline $\begin{array}{l}\text { Lomechusoides strumosus strumo- } \\
\text { sus (FABRICIUs, 1792) }\end{array}$ & 0 & & & ATHW00 \\
\hline $\begin{array}{l}\text { Lomechusoides teres (EPPELSHEIM, } \\
1884 \text { ) }\end{array}$ & $\bigcirc$ & & & Sm04 \\
\hline $\begin{array}{l}\text { Lyprocorrhe anceps (ERICHSON, } \\
1837 \text { ) }\end{array}$ & O & & $8 \mathrm{a}(2)$ & \\
\hline $\begin{array}{l}\text { Meotica exilis (GRAVENHORST, } \\
\text { 1806) }\end{array}$ & 0 & 0 & $39(1), 73(1), 100(1)$ & \\
\hline Meotica arasensis SMETANA, 2004 & O & & $40(1), 130(1)$ & \\
\hline $\begin{array}{l}\text { Mocyta fungi (GRAVENHORST, } \\
1806)^{63)}\end{array}$ & O & & $1(8), 5(1), 9(2), 19(15), 21(1), 39(1), 76(1)$ & \\
\hline Mocyta orbata (ERICHSON, 1837) ${ }^{63)}$ & O & & $9(1), 34(7), 35(18), 36(5), 39(11)$ & \\
\hline${ }^{*}$ Myllaena ambulans Assing, 2018 & $\bigcirc$ & & 19(7), 20(17), 73(2), 76(1) & A18g \\
\hline $\begin{array}{l}\text { Myllaena caucasica EPPELSHEIM, } \\
1880\end{array}$ & O & $\bigcirc$ & $\begin{array}{l}\text { 7(1), 20(12), 20a(1), 23(1), 39(1), 63(4), 73(6), 76(1), } \\
90 \mathrm{a}(1), 100(1), 112(17), 121(7), 121 \mathrm{a}(5), 125(23), \\
126(2), 127(5), 130(5), 181(1), 199 \mathrm{a}(1)\end{array}$ & A18g \\
\hline Myllaena infuscata KraATz, 1853 & O & $\bigcirc$ & $63(1), 66 \mathrm{a}(3), 100(5), 112(1), 121 \mathrm{a}(1)$ & A18g \\
\hline $\begin{array}{l}\text { Myllaena intermedia ERICHSON, } \\
1837\end{array}$ & $\bigcirc$ & & $8 \mathrm{~b}(7), 36(2), 180(2), 190(2), 199(1)$ & A18g \\
\hline $\begin{array}{l}\text { Myrmoecia reitteri (EPPELSHEIM, } \\
1881 \text { ) }\end{array}$ & $\bigcirc$ & & & A17a, IK60 \\
\hline $\begin{array}{l}\text { Myrmoecia urartu (IABLOKOFF- } \\
\text { KHNZORIAN, 1962) }\end{array}$ & 0 & & & A19a, IK62 \\
\hline $\begin{array}{l}\text { Nehemitropia lividipennis (MAN- } \\
\text { NERHEIM, 1830) }\end{array}$ & $\bigcirc$ & O & $36(4), 103(1), 106(2)$ & E30 \\
\hline Ocalea alutacea EpPELSHEIM, 1878 & O & O & $68(16), 101 \mathrm{a}(1), 105(1)$ & \\
\hline Ocalea badia ERICHSON, 1837 & O & & $73(1)$ & \\
\hline $\begin{array}{l}\text { Ocalea dubia MotschuLSKY, } \\
1860^{62)}\end{array}$ & O & O & $\begin{array}{l}58(4), 93(1), 100(4), 101(1), 101 \mathrm{a}(8), 105(7), 109(2), \\
110(14), \quad 112(82), \quad 113(75), \quad 114(120), 115(10), \\
117(10), 148(1)\end{array}$ & \\
\hline Ocalea minor EPPELSHEIM, 1878 & O & ○ & $\begin{array}{l}9(24), 11 \mathrm{a}(1), 19(1), 20(1), 22(1), 23(4), 32(18), \\
39(12), 45(1), 48(115), 49(12), 50(8), 73(17), 75(2), \\
76(50), 77(41), 81(3), 81 \mathrm{a}(11), 82(5), 83(2), 91(1), \\
100(1), 104(3), 105(19), 106(1), 109(1), 110(1), \\
112(2), 114(14), 116(2), 118(3), 119(2), 121(8), \\
121 \mathrm{a}(4), 123(1), 125(11), 126(17), 129(1)\end{array}$ & \\
\hline $\begin{array}{l}\text { Oligota pumilio KIESENWETTER, } \\
1858\end{array}$ & O & & $48(2), 50(1), 70(1), 81 \mathrm{a}(4)$ & \\
\hline $\begin{array}{l}\text { Oligota pusillima (GRAVENHORST, } \\
\text { 1806) }\end{array}$ & O & & $1(1), 11 \mathrm{a}(1), 19(1), 39(8)$ & \\
\hline $\begin{array}{l}\text { Oxypoda abdominalis (MANNER- } \\
\text { HEIM, 1832) }\end{array}$ & $\bigcirc$ & & $1(1), 28(1), 36(1), 166(4)$ & A18c \\
\hline $\begin{array}{l}\text { Oxypodaalternans(GRAVENHORST, } \\
\text { 1802) }\end{array}$ & $\bigcirc$ & O & $22(1), 55(2), 57(18), 69(4), 114(2), 153(1), 170(4)$ & A18c, Ko46 \\
\hline Oxypoda articollis Assing, 2016 & $\bigcirc$ & & $63(1), 69(2)$ & A $18 \mathrm{c}$ \\
\hline
\end{tabular}




\begin{tabular}{|c|c|c|c|c|}
\hline Species & AR & NK & Localities/Samples & References \\
\hline $\begin{array}{l}\text { Oxypoda brevicornis (STEPHENS, } \\
\text { 1832) }\end{array}$ & O & 0 & $\begin{array}{l}4(1), 7(1), 8(1), 9(2), 11 b(2), 13(1), 22(1), 32(1), \\
39(7), 40(1), 45(3), 46(5), 48(4), 49(16), 54(4), \\
63(4), 64(1), 66 a(1), 67(12), 68(3), 70(1), 73(1), \\
74(4), 75(1), 76(6), 77(1), 80 a(1), 81(6), 81 a(5), \\
90(1), 90 a(8), 92(1), 105(7), 106(1), 110(3), 113(3), \\
\begin{array}{l}114(5), 118(6), 121(1), 121 a(3), 123(1), 125(4), \\
126(13), 127(11), 181(2), 206(1)\end{array}\end{array}$ & A18c \\
\hline $\begin{array}{l}\text { Oxypoda caucasica BERNHAUER, } \\
1902\end{array}$ & O & O & $\begin{array}{l}6(1), 11(6), 11 \mathrm{a}(3), 11 \mathrm{~b}(1), 12(9), 22(1), 31(1), \\
32(1), 33(3), 53(6), 58(2), 60(2), 62(2), 63(7), \\
66 \mathrm{a}(3), 66 \mathrm{~b}(8), 68(7), 68 \mathrm{a}(4), 70(1), 92(1), 95(4), \\
101(2), 102(3), 105(13), 106(3), 110(2), 112(2), \\
113(1), 118(24), 118 \mathrm{a}(2), 119(5), 125(1), 149(3), \\
166(3), 170(2), 172(1), 196 \mathrm{a}(1), 199 \mathrm{a}(2)\end{array}$ & \\
\hline $\begin{array}{l}\text { Oxypoda ferruginea ERICHSON, } \\
1839\end{array}$ & O & & $8 \mathrm{a}(1), 39(8), 49(2), 53(3), 54(2), 64(1), 77(2)$ & A18c \\
\hline Oxypoda flavicornis KraAtz, 1856 & O & & $11 \mathrm{a}(5), 92(2)$ & A18c \\
\hline${ }^{\star}$ Oxypoda flexa Assing, 2016 & O & & $9(14(, 9 \mathrm{a}(4), 54(1), 61(2), 76(4)$ & $\mathrm{A} 16 \mathrm{c}, \mathrm{A} 18 \mathrm{c}$ \\
\hline Oxypoda formosa KRAATz, 1856 & O & & 57(1), 70(3) & A18c \\
\hline $\begin{array}{l}{ }_{2 *}^{*} \text { Oxypoda grandecristata AssiNG, } \\
2016\end{array}$ & $\bigcirc$ & & $11(22), 11 \mathrm{a}(11), 11 \mathrm{~b}(4)$ & A16c \\
\hline $\begin{array}{l}\text { Oxypoda haemorrhoa (MANNER- } \\
\text { HEIM, 1830) }\end{array}$ & O & 0 & 20(1), 63(8), 89a(6), 109a(2), 191a(1) & A18c, E30 \\
\hline Oxypoda ignorata ZERCHE, 1996 & O & 0 & $\begin{array}{l}22(4), 39(2), 49(1), 54(2), 63(1), 64(1), 67(3), \\
80 b(1), 86(3), 112(1), 114(68), 116(1), 119(1), \\
123(1), 1135(7), 162(2)\end{array}$ & A18c \\
\hline${ }^{*}$ Oxypoda infissoides Assing, 2016 & O & 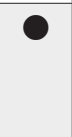 & $\begin{array}{l}9(2), 11 b(1), 12(13), 13(1), 22(1), 39(121), 50(29) \\
89 b(1), 95(1), 96(2), 97(1), 114(5), 118(2), 118 \mathrm{a}(4), \\
121(1), 121 a(5), 126(34), 129(6), 131(2), 167(1)\end{array}$ & A16c, A18c \\
\hline Oxypoda lentula ERICHsON, 1837 & 0 & & $16(1), 85 c(2), 128(3)$ & \\
\hline $\begin{array}{l}{ }^{*} \text { Oxypoda levipunctata Assing, } \\
2016\end{array}$ & O & & $21(1)$ & A16c \\
\hline $\begin{array}{l}\text { Oxypoda longipes Mulsant \& REY, } \\
1861\end{array}$ & O & & $42(2), 46(1), 48(4), 49(1), 67(1), 148(12), 149(1)$ & $\mathrm{A} 18 \mathrm{c}$ \\
\hline $\begin{array}{l}\text { Oxypoda obscuricollis Assing, } \\
2007\end{array}$ & 0 & & $134 \mathrm{~b}(1)$ & \\
\hline Oxypoda recta Assing, 2006 & O & 0 & $\begin{array}{l}3 \mathrm{a}(2), 10(1), 13(1), 16(1), 41(1), 46(1), 47(2), 49 \mathrm{a}(1) \\
64(24), 65(1), 73(5), 80 \mathrm{~b}(1), 87(1), 103(1), 104(1) \\
194(4), 200(1)\end{array}$ & $\mathrm{A} 18 \mathrm{c}$ \\
\hline Oxypoda soror THomson, 1855 & $\bigcirc$ & & $6(1), 47(40), 75(1)$ & A18c \\
\hline Oxypoda spaethi BERNHAUER, 1901 & O & & $167(1)$ & \\
\hline${ }^{\star}$ Oxypoda subplicata Assing, 2016 & O & & $\begin{array}{l}30(100), 31(73), 33(88), 70(1), 77(2), 81(8), 90 a(1) \\
118(24), 123(2), 124(10), 125(8)\end{array}$ & A16c, A18c \\
\hline${ }^{*}$ Oxypoda verminata Assing, 2018 & $\bigcirc$ & & $48(7), 49(1), 50(4)$ & A18c \\
\hline Oxypoda vicina KRAATz, 1858 & $\mathrm{O}$ & 0 & $\begin{array}{l}\text { 1(1), 9(41), 9a(2), 19(9), 20(1), 39(11), 48(7), 50(46), } \\
54(2), 76(6), 80 \mathrm{a}(1), 82(1), 113(1), 126(30)\end{array}$ & A18c \\
\hline Oxypoda vittata MÄRKEL, 1842 & O & 0 & $\begin{array}{l}\text { 8(1), 9(1), 11(1), 31(1), 48(1), 57(1), 60(1), 61(2), } \\
69(1), 114(4), 126(1), 133(1), 136(2), 148 b(1), \\
149(2), 151(3), 155(4), 156(7), 172(1)\end{array}$ & $\begin{array}{l}\text { ATHW00, } \\
\text { A18c }\end{array}$ \\
\hline
\end{tabular}




\begin{tabular}{|c|c|c|c|c|}
\hline Species & AR & NK & Localities/Samples & References \\
\hline $\begin{array}{l}\text { Parocyusa rubicunda (ERICHSON, } \\
1837)^{36)}\end{array}$ & $\bullet$ & $\bullet$ & $\begin{array}{l}\text { 7(1), 9(5), 19(6), 33(2), 39(12), 41(4), 67(8), 73(12), } \\
\text { 75(4), 76(2), 101(1), 103(2), 125(7), 126(4), 130(1), } \\
\text { 199(6), 199a(26) }\end{array}$ & \\
\hline $\begin{array}{l}\text { Pella funesta (GravenHoRst, } \\
\text { 1806) }\end{array}$ & O & & $156(3)$ & ATHW00 \\
\hline Pella laeviceps (EppeLsheIm, 1880) & 0 & - & $\begin{array}{l}2(4), 7(3), 50(2), 63(8), 70(1), 71(1), 74(2), 92(1) \\
97(1), 100(1), 105(1), 133(14), 135(4), 136(7), \\
149 a(5)\end{array}$ & $\begin{array}{l}\text { ATHW00, } \\
\text { Ma06 }\end{array}$ \\
\hline Pella lugens (GRAVENHORSt, 1802) & O & $\bullet$ & $100(1), 135(1), 148(4), 156(2)$ & $\begin{array}{l}\text { ATHW00, } \\
\text { Ma06 }\end{array}$ \\
\hline Pella similis (MäRKEL, 1844) & 0 & & $39(1)$ & \\
\hline $\begin{array}{l}\text { Piochardia reitteri (WASMANN, } \\
\text { 1894) }\end{array}$ & 0 & & & A99, ATHW00 \\
\hline $\begin{array}{l}\text { Poromniusa procidua (ERICHSON, } \\
\text { 1837) }\end{array}$ & 0 & & $21(1)$ & \\
\hline $\begin{array}{l}\text { Pronomaea araxicola ReITTER, } \\
1898\end{array}$ & $\bullet$ & & & A07b, IK57b ${ }^{13)}$ \\
\hline $\begin{array}{l}\text { Pronomaea flavirostris SEMENOV, } \\
2003\end{array}$ & 0 & & $121(2), 121 \mathrm{a}(19), 130(24)$ & \\
\hline $\begin{array}{l}\text { Pronomaea khnzoriani SemENov, } \\
2003\end{array}$ & O & & & A07b, Se03 \\
\hline $\begin{array}{l}\text { Pycnota paradoxa (MULSANT \& } \\
\text { ReY, 1861) }\end{array}$ & 0 & & & ATHW00 ${ }^{14)}$ \\
\hline $\begin{array}{l}\text { Rhopalocerina clavigera (SCRIBA, } \\
\text { 1859) }\end{array}$ & 0 & ○ & $\begin{array}{l}\text { 53(5), 58(2), 88(1), 92(1), 96(19), 97(3), 105(2), } \\
\text { 130(3) }\end{array}$ & \\
\hline $\begin{array}{l}\text { Tachyusa impressa EPPELSHEIM, } \\
1878\end{array}$ & 0 & & 99(7), 161(9), 178(1), 198(1), 199(1) & \\
\hline $\begin{array}{l}{ }^{*} \text { Tachyusa unguis Assing, } \\
\text { spec. nov. }\end{array}$ & $\bullet$ & & 206(5) & ASpp \\
\hline $\begin{array}{l}\text { Thamiaraea cinnamomea } \\
\text { (GraVENHORST, 1802) }\end{array}$ & 0 & & $148 \mathrm{~b}(1), 150(2)$ & \\
\hline $\begin{array}{l}\text { Thiasophila angulata (ERICHSON, } \\
\text { 1837) }\end{array}$ & 0 & & $8 \mathrm{a}(6)$ & \\
\hline $\begin{array}{l}\text { Thinonoma atra (GRAVENHORST, } \\
\text { 1806) }\end{array}$ & 0 & & $85 c(10), 198 \mathrm{a}(1), 199(1)$ & \\
\hline Trichiusa immigrata LoHse, 1984 & 0 & & $80 \mathrm{~b}(1)$ & \\
\hline $\begin{array}{l}\text { Tropimenelytron mirabile (EPPELS- } \\
\text { HEIM, 1884) }\end{array}$ & 0 & & 41(4), 89(1), 121(4), 121a(2), 125(1) & \\
\hline $\begin{array}{l}\text { Tropimenelytron tuberiventre } \\
\text { (EPPELSHEIM, 1880) }\end{array}$ & 0 & & $\begin{array}{l}\text { 1(1), 6(1), 7(2), 8(1), 51(3), 53(2), 55(2), 58(1), } \\
\text { 59(1),62(4),63(13),66b(2),68(2),68a(1),69(1), } \\
70(2), 75(9), 92(1), 180(1)\end{array}$ & $\mathrm{Be} 74^{15)}$ \\
\hline Zyras collaris (PAYKULL, 1800) & 0 & $\bullet$ & $66 \mathrm{a}(2), 78(2), 88(6), 105(1), 112(7), 127(4)$ & \\
\hline $\begin{array}{l}\text { Zyras fulgidus (GRAVENHORST, } \\
\text { 1806) }\end{array}$ & 0 & & 135(1), 183(1), 205(1), c(1) & \\
\hline Zyras haworthi (STEPHENS, 1832) & O & $\bullet$ & 11(3), 66a(1), 81a(1), 88(1), 100(1), 114(2) & Dv84, ATHW00 \\
\hline \multicolumn{5}{|l|}{ Scaphidiinae } \\
\hline $\begin{array}{l}\text { Scaphisoma agaricinum (LiN- } \\
\text { NAEUS, 1758) }\end{array}$ & 0 & & & IK57a \\
\hline
\end{tabular}




\begin{tabular}{|c|c|c|c|c|}
\hline Species & AR & NK & Localities/Samples & References \\
\hline $\begin{array}{l}\text { Scaphisoma balcanicum TAMA- } \\
\text { NINI, } 1954\end{array}$ & $\bigcirc$ & & & Lö70 \\
\hline Scaphisoma simillimum LӧвL, 1970 & O & & & IK85 \\
\hline $\begin{array}{l}\text { Scaphisoma subalpinum subalpi- } \\
\text { num REITTER, } 1880\end{array}$ & $\bigcirc$ & & & IK57a \\
\hline \multicolumn{5}{|l|}{ Oxytelinae } \\
\hline $\begin{array}{l}\text { Anotylus clypeonitens (PANDELLÉ, } \\
\text { 1867) }\end{array}$ & O & O & $36(1), 63(2), 100(2), 105(3), 110(1), 171(6), a c(1)$ & \\
\hline $\begin{array}{l}\text { Anotylus fairmairei (PANDELLÉ, } \\
\text { 1867) }\end{array}$ & 0 & & $44(6), 45(3), \operatorname{ad}(1)$ & \\
\hline $\begin{array}{l}\text { Anotylus gibbulus (EPPELSHEIM, } \\
\text { 1878) }\end{array}$ & $\bigcirc$ & & & HMM79 \\
\hline $\begin{array}{l}{ }^{\star} \text { Anotylus hamatoides SCHÜLKE, } \\
\text { spec. nov. }\end{array}$ & O & & $136(1), 148 \mathrm{c}(2), 150(2), 151 \mathrm{a}(1)$ & \\
\hline $\begin{array}{l}\text { Anotylus hammondi SCHÜLKE, } \\
2009\end{array}$ & ○ & & $4(1)$, ae$(1)$, af(1) & \\
\hline $\begin{array}{l}\text { Anotylus intricatus (ERICHSON, } \\
1840 \text { ) }\end{array}$ & O & & $\operatorname{ag}(1)$ & \\
\hline $\begin{array}{l}\text { Anotylus inustus (GRAVENHORST, } \\
1806 \text { ) }\end{array}$ & 0 & & $8(1)$ & \\
\hline $\begin{array}{l}\text { Anotylus khachikovi GILDENKov, } \\
2017\end{array}$ & O & O & 64(1), 74(3), 81(1), 82(3), 105(52), 109(1), 126(5) & \\
\hline Anotylus mutator (LoHsE, 1963) & O & & $\begin{array}{l}\text { 7(3), 15(2), 63(1), 135(4), 136(6), 148c(5), 149a(9), } \\
150(3), 150 \mathrm{a}(21), 151(3), 151 \mathrm{a}(9), 151 \mathrm{~b}(6)\end{array}$ & \\
\hline $\begin{array}{l}\text { Anotylus nitidulus (GRAVENHORST, } \\
\text { 1802) }\end{array}$ & $\bigcirc$ & O & $8 \mathrm{a}(1), 47(1), 49(3), 49 \mathrm{a}(1), 104(1), 186(1), 204 \mathrm{a}(2)$ & Sm04 \\
\hline $\begin{array}{l}\text { Anotylus pumilus (ERICHSON, } \\
\text { 1839) }\end{array}$ & O & & & E30 \\
\hline $\begin{array}{l}\text { Anotylus rugosus (FABRICIUS, } \\
1775 \text { ) }\end{array}$ & $\bigcirc$ & & $20(6), 36(1), 199(1), 199 a(1)$ & $\mathrm{Sm} 04$ \\
\hline $\begin{array}{l}\text { Anotylus sculpturatus (GRAVEN- } \\
\text { HORST, 1806) }\end{array}$ & $\bigcirc$ & 0 & $\begin{array}{l}22 \mathrm{a}(1), 30(2), 32(4), 33(1), 53(4), 61(1), 66(5), \\
66 \mathrm{a}(1), 66 \mathrm{~b}(1), 81(4), 81 \mathrm{a}(14), 89(6), 90(1), 191 \mathrm{a}(1), \\
105(31), 109(3), 110(1), 112(1), 113(4), 114(4), \\
115(2), 118(1), 125(5), 126(16), 127(3), 148 \mathrm{a}(12), \\
148 \mathrm{~b}(28), 149 \mathrm{~b}(9), 150 \mathrm{a}(1), 151 \mathrm{a}(1), 152(4)\end{array}$ & Sm04 \\
\hline $\begin{array}{l}\text { Anotylus strigifrons (НоснНUTH, } \\
1849 \text { ) }\end{array}$ & ○ & & $\begin{array}{l}\text { 53(1), 63(2), 67(5), 73(1), 74(1), 77(1), ah(1), ai(1), } \\
\text { aj(1), ak(2) }\end{array}$ & \\
\hline $\begin{array}{l}\text { Anotylus tetracarinatus (BLOCK, } \\
\text { 1799) }\end{array}$ & $\bigcirc$ & & $45(1), 58(1), 81 \mathrm{a}(1), 96(1)$ & E30 \\
\hline $\begin{array}{l}\text { Anotylus tetratoma (CzWALINA, } \\
1871 \text { ) }\end{array}$ & ○ & & $44(3), 81 \mathrm{a}(1)$ & \\
\hline $\begin{array}{l}\text { Aploderus caelatus (GRAVENHORST, } \\
\text { 1802) }\end{array}$ & O & & $53(1)$ & \\
\hline $\begin{array}{l}\text { Bledius gallicus (GRAVENHORST, } \\
\text { 1806) }\end{array}$ & $\mathrm{O}$ & & & $\mathrm{E} 30^{16)}$ \\
\hline Bledius debilis ERICHSON, 1840 & & $\bigcirc$ & & Hh49 \\
\hline Bledius hinnulus ERICHSON, 1840 & O & & & E30 \\
\hline Bledius minniensis HERMAN, 1986 & $\bigcirc$ & & & H86 \\
\hline
\end{tabular}




\begin{tabular}{|c|c|c|c|c|}
\hline Species & AR & NK & Localities/Samples & References \\
\hline Bledius roettgeni BERNHAUER, 1928 & O & & & Sm04 \\
\hline Bledius spectabilis KraAtz, 1857 & O & & $\mathrm{a}(4)$ & E30 \\
\hline Bledius tibialis HeER, 1839 & $\bullet$ & 0 & $\mathrm{al}(1)$ & Ko46 ${ }^{37)}$ \\
\hline Bledius tricornis (Herbst, 1784) & $\bullet$ & & $a(2), d(1)$ & \\
\hline $\begin{array}{l}\text { Carpelimus bilineatus STEPHENS, } \\
1834\end{array}$ & O & & 177(2), 199(1), 199a(1), a(1), b(1), d(1) & Sm04 \\
\hline $\begin{array}{l}\text { Carpelimus corticinus (GRAVEN- } \\
\text { HORST, 1806) }\end{array}$ & O & & 16(1), 20(1), 66a(3), 85c(2), 128(1), 130(1), 177(6) & Gi15 \\
\hline $\begin{array}{l}\text { Carpelimus elongatulus elongatulus } \\
\text { (ERICHSON, 1839) }\end{array}$ & O & & & Sm04 \\
\hline Carpelimus erichsoni (SHARP, 1871) & O & & 196(1) & \\
\hline $\begin{array}{l}\text { Carpelimus exiguus (ERICHSON, } \\
\text { 1839) }\end{array}$ & O & & & Sm04 \\
\hline $\begin{array}{l}\text { Carpelimus fuliginosus (GRAVEN- } \\
\text { HORST, 1802) }\end{array}$ & O & & & Sm04 \\
\hline $\begin{array}{l}\text { Carpelimus gracilis (MANNERHEIM, } \\
\text { 1830) }\end{array}$ & O & & & Gi01 \\
\hline $\begin{array}{l}\text { Carpelimus gusarovi GILDENKov, } \\
1997\end{array}$ & $\bullet$ & & $\mathrm{g}(1)$ & \\
\hline Carpelimus heydeni (KLIMA, 1904) & $\bullet$ & & 212(12) & \\
\hline $\begin{array}{l}\text { Carpelimus impressus (LACOR- } \\
\text { DAIRE, 1835) }\end{array}$ & O & & & Sm04 \\
\hline $\begin{array}{l}\text { Carpelimus manchuricus subtilicor- } \\
\text { nis (RouBAL, 1946) }\end{array}$ & 0 & & $53(4), 199 a(2)$ & \\
\hline $\begin{array}{l}\text { Carpelimus rivularis (Мот- } \\
\text { SCHULSKY, 1860) }\end{array}$ & $\bigcirc$ & & $66 \mathrm{a}(1)$ & Sm04 \\
\hline $\begin{array}{l}\text { Carpelimus similis (SMETANA, } \\
\text { 1967) }\end{array}$ & 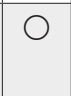 & & & G01, G09 \\
\hline $\begin{array}{l}\text { Coprophilus pennifer (Мот- } \\
\text { scHULSKY, 1845) }\end{array}$ & $\bigcirc$ & & $\mathrm{e}(1)$ & ATHW00 \\
\hline $\begin{array}{l}\text { Coprophilus striatulus (FABRICIUS, } \\
\text { 1792) }\end{array}$ & 0 & & $181(1)$ & \\
\hline $\begin{array}{l}\text { Deleaster dichrous (GRAVENHORST, } \\
\text { 1802) }\end{array}$ & O & & $134(1), f(1)$ & Fa71 ${ }^{17)}$ \\
\hline Oxytelus fulvipes ERICHSON, 1839 & $\bigcirc$ & & & E30 \\
\hline $\begin{array}{l}\text { Oxytelus laqueatus (MARSHAM, } \\
1802 \text { ) }\end{array}$ & $O$ & & $8(3), 59(1), 148 \mathrm{a}(1)$ & Sm04 \\
\hline Oxytelus piceus (Linnaeus, 1767) & $\bigcirc$ & & $44(1)$ & Sm04 \\
\hline $\begin{array}{l}\text { Oxytelus sculptus GravenHORST, } \\
1806\end{array}$ & 0 & & & Sm04 \\
\hline $\begin{array}{l}\text { Platystethus alutaceus Tномsоn, } \\
1861\end{array}$ & 0 & & $21(1), 128(106)$ & \\
\hline Platystethus capito HeER, 1839 & 0 & ○ & $\begin{array}{l}\text { 49(1), 54(1), 58(3), 63(12), 66a(5), 67(2), 72(1), } \\
\text { 73(3), 76(3), 81a(1), 88(1), 93a(38), 94(1), 99(41), } \\
\text { 100(6), 127(2), 131(1), 195(1), 197(1), 199a(4), } \\
\text { 202(1), 204a(2), 210(11), ah(1), am(1), an(1), ao(1), } \\
\text { ap(1), aq(1) }\end{array}$ & \\
\hline
\end{tabular}




\begin{tabular}{|c|c|c|c|c|}
\hline Species & AR & NK & Localities/Samples & References \\
\hline $\begin{array}{l}\text { Platystethus cornutus (GRAVEN- } \\
\text { HORST, 1802) }\end{array}$ & $\bigcirc$ & & $177(4), 190(1)$ & Sm04 \\
\hline $\begin{array}{l}\text { Platystethus gildenkovi KHACHI- } \\
\text { KOV, } 2013\end{array}$ & 0 & & $44(1)$ & \\
\hline $\begin{array}{l}\text { Platystethus nitens (C. R. SAHL- } \\
\text { BERG, 1832) }\end{array}$ & O & O & $\begin{array}{l}41(2), 45(3), 49(1), 67(1), 100(1), 112(1), 177(6), \\
184(4), 206(1)\end{array}$ & $\mathrm{Sm} 04$ \\
\hline $\begin{array}{l}\text { Platystethus rufospinus } \mathrm{HocH}^{-} \\
\text {HUTH, } 1851\end{array}$ & 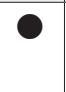 & & $184(2), 186(1)$ & \\
\hline $\begin{array}{l}\text { Platystethus rugifrons BERNHAUER, } \\
1900\end{array}$ & O & & & $\mathrm{Sm} 04$ \\
\hline $\begin{array}{l}\text { Platystethus spinosus ERICHSON, } \\
1840\end{array}$ & O & & & E30 \\
\hline $\begin{array}{l}\text { Syntomium aeneum (MüLLER, } \\
\text { 1821) }\end{array}$ & O & & $52(3), 153(1)$ & \\
\hline $\begin{array}{l}\text { Thinodromus dilaticollis (EPPELS- } \\
\text { HEIM, 1884) }\end{array}$ & ○ & O & $\begin{array}{l}39(1), \quad 100(2), \quad 109(1), \quad 117(3), \quad 121 \mathrm{a}(2), \quad 126(1), \\
188(4), 205(1), 206(6)\end{array}$ & Gi00 \\
\hline $\begin{array}{l}\text { Thinodromus kiesenwetterii } \\
\text { (Носннштн, 1851) }\end{array}$ & O & & & Gi00 \\
\hline $\begin{array}{l}\text { Thinodromus mannerheimii } \\
\text { (KoLENATI, 1846) }\end{array}$ & $\mathrm{O}$ & & & Gi00 \\
\hline $\begin{array}{l}\text { Thinodromus motschulskyi (HocH- } \\
\text { HUTH, 1860) }\end{array}$ & 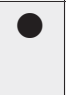 & & 199(3), 199a(3) & \\
\hline $\begin{array}{l}\text { Thinodromus transversalis } \\
\text { (WoLLASTON, 1857) }\end{array}$ & ○ & & $212(1)$ & \\
\hline \multicolumn{5}{|l|}{ Oxyporinae } \\
\hline $\begin{array}{l}\text { Oxyporus rufus rufus (LinnaEus, } \\
1758 \text { ) }\end{array}$ & ○ & & $53(1), 70(4), 133(1)$ & \\
\hline \multicolumn{5}{|l|}{ Sten ina e } \\
\hline $\begin{array}{l}\text { Dianous coerulescens elegans } \\
\text { IABLOKOFF-KHNZORIAN, } 1957\end{array}$ & $\mathrm{O}$ & & $209(1)$ & IK57b \\
\hline Dianous ponticus FAGEL, 1963 & $\bigcirc$ & & $181(1)$ & P16 \\
\hline Stenus arabicus SAULCY, 1865 & ○ & & $183(2)$ & \\
\hline Stenus araxis Ryvkin, 1990 & $\bigcirc$ & & & Ry90 \\
\hline Stenus argus Gravenhorst, 1806 & $\bigcirc$ & & 19(1), 201(2), 202(1) & Sm04 ${ }^{50)}$ \\
\hline Stenus armeniacus Puthz, 1967 & $\mathrm{O}$ & & & Pu67 \\
\hline Stenus ater MANNERHEIM, 1830 & $\mathrm{O}$ & & & Ry90 \\
\hline Stenus atratulus ERICHSON, 1839 & O & O & $\begin{array}{l}50(2), 61(1), 66(1), 66 a(2), 73(1), 76(3), 103(1), \\
106(1), 107(1), 126(4)\end{array}$ & Ry90 \\
\hline Stenus biguttatus LinnaEus, 1758 & ○ & & & Ry90 \\
\hline $\begin{array}{l}\text { Stenus bimaculatus GYLLENHAL, } \\
1810\end{array}$ & O & & & Ry90 \\
\hline Stenus binotatus LJUNGH, 1804 & O & & & Sm04 \\
\hline $\begin{array}{l}\text { Stenus callidus BAUdi di SELVE, } \\
1848\end{array}$ & 0 & & 19(1), 20(4), 210(1) & $\begin{array}{l}\text { Hr90 }{ }^{47)}, \mathrm{P} 08, \\
\text { Ry90 }\end{array}$ \\
\hline Stenus caspius Puthz, 1972 & ○ & & $43(1)$ & Ry90 \\
\hline Stenus cautus ERICHSON, 1839 & 0 & & 192(1) & \\
\hline
\end{tabular}




\begin{tabular}{|c|c|c|c|c|}
\hline Species & AR & NK & Localities/Samples & References \\
\hline $\begin{array}{l}\text { Stenus cephallenicus BERNHAUER, } \\
1913\end{array}$ & O & & $\begin{array}{l}\text { 9(3), 20(5), 40(1), 67(1), 76(5), 77(9), 82(19), 82a(8), } \\
93(5), 119(1), 126(16), 130(7), 131(2)\end{array}$ & \\
\hline $\begin{array}{l}\text { Stenus cicindeloides (SCHALLER, } \\
1783 \text { ) }\end{array}$ & $\mathrm{O}$ & & $206(3)$ & $\mathrm{Sm} 04^{50)}$ \\
\hline Stenus claritarsis Puthz, 1971 & $\mathrm{O}$ & & $183(2)$ & Ry90 \\
\hline Stenus clavicornis SCOPOLI, 1763 & O & O & $\begin{array}{l}47(14), 64(3), 66 a(1), 67(1), 78(3), 79(2), 89(1), \\
89 b(1), 98(1), 100(1), 101(2), 101 a(1), 107(2), \\
109(1), 127(1), 130(4), 183(3)\end{array}$ & Ry90 \\
\hline $\begin{array}{l}\text { Stenus coarcticollis coarcticollis } \\
\text { EPPELSHEIM, } 1890\end{array}$ & O & O & $\begin{array}{l}1(4), 6(1), 7(7), 11(25), 11 \mathrm{a}(11), 11 \mathrm{~b}(18), 12(5), \\
15(10), 30(1), 33(6), 39(4), 50(15), 51(5), 53(6), \\
58(2), 60(1), 63(1), 66(4), 66 \mathrm{~b}(1), 68(1), 71(6), \\
72(6), 75(2), 82(7), 82 \mathrm{a}(17), 83(5), 88(1), 89(18), \\
89 \mathrm{~b}(21), 90 \mathrm{a}(1), 91(1), 92(4), 95(1), 96(2), 97(11), \\
103(7), 105(2), 113(1), 118(12), 118(5), 119(6), \\
125(17), 126(50), 131(13), 135(2), 136(1), 151 \mathrm{a}(1), \\
180(1)\end{array}$ & Ry90 \\
\hline $\begin{array}{l}\text { Stenus comma comma LECONTE, } \\
1863\end{array}$ & O & O & $14(1)$ & Ry90 \\
\hline $\begin{array}{l}\text { Stenus erythrocnemus EPPELSHEIM, } \\
1884\end{array}$ & O & $\mathrm{O}$ & 66a(1), 68(1), 112(1), 126(1) & A06, Ry90 \\
\hline $\begin{array}{l}\text { Stenus ganglbaueri BERNHAUER, } \\
1905\end{array}$ & O & & $\mathrm{n}(1)$ & Sm04 ${ }^{50)}$ \\
\hline Stenus humilis ERICHSON, 1839 & O & $\mathrm{O}$ & $\begin{array}{l}3 a(4), 6(5), 41(4), 42(3), 43(1), 45(1), 47(1), 48(1), \\
51(1), 53(11), 54(6), 57(3), 58(1), 63(1), 66(3), \\
66 a(5), 67(4), 68(2), 68 a(1), 70(2), 88(11), 98(18), \\
100(3), 101(12), 102(2), 105(5), 106(4), 107(2), \\
109(1), 117(3), 136(1), 180(1)\end{array}$ & P83, Ry90 \\
\hline Stenus ignotus EPPELSHEIM, 1890 & O & & & Ry90 \\
\hline Stenus juno (PAYKULL, 1789) & $\bigcirc$ & & 41(3), 198(1), 201(4) & $\mathrm{Sm} 04^{50)}$ \\
\hline $\begin{array}{l}\text { Stenus machulkai HROMÁDKA, } \\
1977\end{array}$ & O & O & $\begin{array}{l}19(3), 20(11), 22(7), 39(2), 77(30), 81(749), \\
81 \mathrm{a}(677), 82(4), 88(9), 89(7), 89 \mathrm{~b}(9), 90(6), 90 \mathrm{a}(6), \\
91(11), 103(1), 105(3), 106(2), 107(4), 109(27), \\
110(6), 112(2), 113(1), 114(7), 117(1), 118(3), \\
121 \mathrm{a}(1), 126(65), 127(13), 128(2), 129(1), 130(33), \\
131(1)\end{array}$ & Ry90 \\
\hline Stenus medus Puthz, 1981 & O & O & $\begin{array}{l}50(1), 76(2), 114(38), 115(2), \quad 118(1), \quad 121(1), \\
121 \mathrm{a}(20), 125(1), 130(46)\end{array}$ & P81, Ry90 \\
\hline Stenus morio GRAVENHORST, 1806 & O & & $63(1)$ & Ep78 \\
\hline Stenus nanus Stephens, 1833 & O & & $46(1), 54(2), 67(1), 73(1), 94(1)$ & \\
\hline Stenus nigritulus GylLENHAL, 1827 & O & 0 & $103(2), 104(1)$ & P12 \\
\hline Stenus nodipes Puthz, 1972 & O & & & P09 \\
\hline $\begin{array}{l}\text { Stenus ochropus KIESENWETTER, } \\
1858\end{array}$ & O & $\mathrm{O}$ & $\begin{array}{l}22(83), 22 \mathrm{a}(4), 26(57), 30(23), 31(2), 32(50), 34(2), \\
35(26), 39(10), 71(1), 78(6), 79(7), 81(140), 81 \mathrm{a}(51), \\
88(46), 89(3), 89 \mathrm{~b}(1), 97(2), 98(51), 100(26), \\
101(23), 101 \mathrm{a}(8), 102(6), 103(5), 109(1), 112(105), \\
114(260), 115(1), 116(24), 117(7), 118(14), 118 \mathrm{a}(7), \\
119(7), 121(6), 121 \mathrm{a}(23), 124(2), 126(24), 127(27), \\
135(1), 136(2), 137(2), 149(1), 149 \mathrm{a}(1), 150 \mathrm{a}(1), \\
155(1), 167(1), 170(1)\end{array}$ & Ry90 \\
\hline Stenus peripherus KoRgE, 1971 & $\bigcirc$ & & & P08 \\
\hline
\end{tabular}




\begin{tabular}{|c|c|c|c|c|}
\hline Species & AR & NK & Localities/Samples & References \\
\hline Stenus piscator SAULCY, 1865 & $\bigcirc$ & & & Ry90 \\
\hline Stenus prometheus Puthz, 1967 & O & & $\begin{array}{l}4(1), 11(3), 11 \mathrm{~b}(2), 13(5), 16(15), 45(1), 49(1) \\
49 \mathrm{a}(1), 70(1), 83(3), 119(1), 123(1)\end{array}$ & P67, P03, Ry90 \\
\hline Stenus proprius BENICK, 1921 & O & & $31(1)$ & \\
\hline $\begin{array}{l}\text { Stenus providus providus ERICH- } \\
\text { sON, } 1839\end{array}$ & $\bigcirc$ & & $20(1), 81 \mathrm{a}(1), 130(6)$ & Ry90 \\
\hline Stenus similis (HERBST, 1784) & $\bigcirc$ & & $\begin{array}{l}6(1), 30(1), 47(2), 76(1), 81 \mathrm{a}(1), 82(1), 82 \mathrm{a}(1), \\
88(2), 125(1)\end{array}$ & Ry90 \\
\hline Stenus skoraszewskyi KoRGE, 1971 & $\bigcirc$ & & $85 a(1), 85 c(14), 94 a(2), 128(1), 201(6)$ & Sm04 ${ }^{50)}$ \\
\hline Stenus stigmula ERICHSON, 1840 & O & & & P81 \\
\hline Stenus subditus BENICK, 1920 & 0 & & & P83 \\
\hline Stenus turk Puthz, 1972 & $\bigcirc$ & $\bigcirc$ & $90 a(1), 114(381), 130(1)$ & P72b ${ }^{18)}$, Ry90 \\
\hline \multicolumn{5}{|l|}{ Scydmaeninae } \\
\hline $\begin{array}{l}\text { Cephennium perispinctum } \\
\text { perispinctum KoLENATI, } 1846\end{array}$ & O & & & VB04 \\
\hline $\begin{array}{l}{ }^{* *} \text { Euconnus karabakhus MEY- } \\
\text { вонм, spec. nov. }\end{array}$ & & O & $98(2), 101(3), 102(1)$ & \\
\hline $\begin{array}{l}\text { Euconnus lalvarensis IABLOKOFF- } \\
\text { KHNZORIAN, } 1964\end{array}$ & $\bigcirc$ & O & $3 a(1), 51(1), 53(1), 55(1), 66(1), 75(1), 88(1), 112(1)$ & IK64a \\
\hline $\begin{array}{l}{ }^{*} \text { Euconnus longilaminatus MEY- } \\
\text { вонм, spec. nov. }\end{array}$ & 0 & & $70(11)$ & \\
\hline $\begin{array}{l}{ }^{* *} \text { Euconnus tavushus Меувонм, } \\
\text { spec. nov. }\end{array}$ & 0 & & $66 a(20)$ & \\
\hline $\begin{array}{l}\text { Euconnus wetterhallii (GYLLEN- } \\
\text { HAL, 1813) }\end{array}$ & ○ & & $66 a(1)$ & \\
\hline $\begin{array}{l}\text { Neuraphes angulatus (MÜLLER \& } \\
\text { KunZE, 1822) }\end{array}$ & O & O & $7(1), 8(1), 11 b(1), 92(1), 105(5)$ & \\
\hline $\begin{array}{l}{ }^{* *} \text { Neuraphes gomarantsus MEY- } \\
\text { вОНM, spec. nov. }\end{array}$ & 0 & & $30(3), 31(1)$ & \\
\hline $\begin{array}{l}{ }^{* *} \text { Neuraphes syunikus МЕувонм, } \\
\text { spec. nov. }\end{array}$ & ○ & & $81 \mathrm{a}(1), 118(14), 118 \mathrm{a}(2), 119(1)$ & \\
\hline $\begin{array}{l}\text { Scydmaenus cornutus MoT- } \\
\text { sCHULSKY, } 1844\end{array}$ & O & & & E30 \\
\hline $\begin{array}{l}\text { Scydmoraphes helvolus (SCHAUM, } \\
1844 \text { ) }\end{array}$ & 0 & & $81 \mathrm{a}(1), 118(1)$ & \\
\hline $\begin{array}{l}\text { Scydmoraphes yermolowi (SAULCY, } \\
1878 \text { ) }\end{array}$ & $\bigcirc$ & & $66(5), 66 \mathrm{~b}(2), 68(3), 68 \mathrm{a}(1)$ & $\mathrm{Da} 04$ \\
\hline Stenichnus poweri (FowLER, 1884) & O & & 53(3), 57(3), 63(1), 66(4), 66b(7), 77(1) & \\
\hline $\begin{array}{l}\text { Stenichnus pusillus pusillus } \\
\text { (MüLLER \& KunZE, 1822) }\end{array}$ & 0 & & $66(3)$ & \\
\hline $\begin{array}{l}\text { Stenichnus scutellaris (MÜLLER \& } \\
\text { KunZE, 1822) }\end{array}$ & 0 & O & $9(1), 22(1), 103(2)$ & \\
\hline $\begin{array}{l}\text { Stenichnus subseriatus FrANZ, } \\
1960^{59)}\end{array}$ & 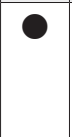 & ○ & $\begin{array}{l}\text { 6(3), 50(3), 51(3), 52(1), 55(2), 57(1), 58(3), 59(2), } \\
\text { 61(1), 62(4), 63(8), 66(2), 68(5), 69(4), 71(2), 98(1), } \\
100(2), 101(1), 105(3), 114(1), 117(1)\end{array}$ & \\
\hline \multicolumn{5}{|l|}{ Pa ederinae } \\
\hline $\begin{array}{l}\text { Achenium caucasicum LAPORTE, } \\
1835\end{array}$ & O & & & AKÖ11 \\
\hline
\end{tabular}




\begin{tabular}{|c|c|c|c|c|}
\hline Species & AR & NK & Localities/Samples & References \\
\hline Achenium debile ERICHSON, 1840 & O & & & Kh37 \\
\hline Achenium humile (NICOLAI, 1822) & O & & & A17a \\
\hline $\begin{array}{l}\text { Achenium quadriceps Eppelsheim, } \\
1889\end{array}$ & $\bullet$ & & 177(4), a(2), i(1) & \\
\hline $\begin{array}{l}\text { Astenus bimaculatus bimaculatus } \\
\text { (ERICHSON, 1840) }\end{array}$ & O & & & E30 \\
\hline Astenus lyonessius (Joy, 1908) & $\bullet$ & $\bullet$ & $\begin{array}{l}\text { 22a(5), 23a(1), 36(1), 38(1), 78(6), 79(15), 80(1), } \\
88(14), 89 \mathrm{~b}(1), 114(10), 115(1), 121 \mathrm{a}(1), 126(5), \\
194(1)\end{array}$ & \\
\hline $\begin{array}{l}\text { Astenus procerus (GRAVENHORST, } \\
1806 \text { ) }\end{array}$ & O & & & $\begin{array}{l}\text { A17a, Am00 19), } \\
\text { IK61 20) }\end{array}$ \\
\hline Astenus rufopacus ReItter, 1909 & O & & $79(1)$ & An17, C60 39) \\
\hline $\begin{array}{l}\text { Homaeotarsus chaudoirii } \mathrm{HoCH}_{-} \\
\text {HUTH, } 1851\end{array}$ & $\bullet$ & & $\mathrm{b}(1)$ & \\
\hline $\begin{array}{l}\text { Lathrobium brunnipes (FABRICIUS, } \\
\text { 1792) }\end{array}$ & O & & & A17a \\
\hline $\begin{array}{l}\text { Lathrobium fulvipenne (GRAVEN- } \\
\text { HORST, 1806) }\end{array}$ & O & & 183(1) & A17a \\
\hline Lathrobium laevipenne HEER, 1839 & $\bullet$ & & $204 \mathrm{a}(4)$ & \\
\hline $\begin{array}{l}\text { Lathrobium longulum GRAVEN- } \\
\text { HORST, } 1802\end{array}$ & O & & $9(1)$ & A17a \\
\hline $\begin{array}{l}\text { Lathrobium permutatum AssiNG, } \\
2009\end{array}$ & $\bullet$ & $\bullet$ & $\begin{array}{l}\text { 33(11), 36(6), 39(2), 50a(1), 56(1), 66(1), 101(1), } \\
\text { 104(1), 112(1), 126(11), 199a(3) }\end{array}$ & \\
\hline $\begin{array}{l}\text { Lathrobium tichomirovae CoIf- } \\
\text { FAIT, } 1981\end{array}$ & $\bullet$ & $\bullet$ & $\begin{array}{l}42(1), 43(1), 50 \mathrm{a}(1), 54(1), 67(3), 81 \mathrm{a}(1), 82(10) \\
90(2), 107(1), 179(3), 206(3)\end{array}$ & \\
\hline $\begin{array}{l}\text { Leptobium gracile (GRAVENHORST, } \\
\text { 1802) }\end{array}$ & 0 & & & A05b \\
\hline $\begin{array}{l}{ }^{*} \text { Lobrathium ancoriferum Assing, } \\
2017\end{array}$ & O & & & A17d \\
\hline $\begin{array}{l}\text { Lobrathium rugipenne (HocH- } \\
\text { HUTH, 1851) }\end{array}$ & O & & 179(1), 181(1), 204a(1) & A17d, Hh51 \\
\hline Luzea graeca (KRAATZ, 1857) & ○ & & & $\mathrm{IK}^{41)}$ \\
\hline $\begin{array}{l}\text { Medon dilutus pythonissa (SAULCY, } \\
\text { 1865) }\end{array}$ & O & & & A18d, C70c ${ }^{40)}$ \\
\hline $\begin{array}{l}\text { Medon exquisitus KIRSHENBLAT, } \\
1951^{42)}\end{array}$ & O & & & $\begin{array}{l}\text { A13a, IK64b, } \\
\text { Ki51 }\end{array}$ \\
\hline Medon fusculoides Coiffait, 1970 & O & $\bullet$ & 112(1), 121(10), 121a(50), 130(1) & C70d \\
\hline Medon maronitus (SAULCY, 1865) & O & & & A18d \\
\hline Medon paradisiacus Assing, 2004 & O & & $26(2), 34(5)$ & A18d \\
\hline${ }^{*}$ Medon sequax Assing, 2004 & O & & $188(3)$ & A04a \\
\hline $\begin{array}{l}\text { Ochthephilum egregium (REITTER, } \\
\text { 1884) }\end{array}$ & $\bullet$ & & $36(11), 66(5), 66 a(3)$ & \\
\hline $\begin{array}{l}\text { Ochthephilum turkestanicum } \\
\text { (KorGE, 1968) }\end{array}$ & $\bullet$ & & 190(1) & \\
\hline $\begin{array}{l}\text { Paederidus rubrothoracicus } \\
\text { (GoEZE, 1777) }\end{array}$ & O & & & Ki32, Sz57 ${ }^{49)}$ \\
\hline
\end{tabular}




\begin{tabular}{|c|c|c|c|c|}
\hline Species & AR & NK & Localities/Samples & References \\
\hline $\begin{array}{l}\text { Paederidus ruficollis (FABRICIUS, } \\
\text { 1777) }\end{array}$ & 0 & & 199(5) & \\
\hline $\begin{array}{l}\text { Paederus fuscipes fuscipes CuRTIs, } \\
1826\end{array}$ & O & & 177(5), 190(1), 203a(3), d(2) & An18, Ki32 \\
\hline $\begin{array}{l}\text { Paederus littoralis GRAVENHORST, } \\
1802\end{array}$ & O & 0 & $\begin{array}{l}7 \mathrm{a}(8), 13(1), 37(1), 63(1), 66 \mathrm{a}(1), 78(1), 79(1) \\
81 \mathrm{a}(1), 88(1), 99(1), 105(1), 109(2), 111(1), 112(2), \\
134 \mathrm{a}(1), 137(1), 141(1), 203 \mathrm{a}(1), \mathrm{h}(1)\end{array}$ & An18 \\
\hline $\begin{array}{l}\text { Paederus riparius (LINNAEUs, } \\
1758 \text { ) }\end{array}$ & O & & & Ki32 \\
\hline $\begin{array}{l}\text { Rugilus armeniacus (CoIfFAIT, } \\
\text { 1970) }\end{array}$ & O & 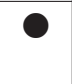 & 101(1), 121a(1), 199a(1) & A17a, C70a \\
\hline Rugilus longicollis (FAUveL, 1900) & 0 & 0 & $\begin{array}{l}15(2), 30(6), 32(13), 36(2), 63(1), 66(1), 66 a(2), \\
80 \mathrm{~b}(22), 88(1), 89(1), 98(2), 101(1), 103(1), 109(2), \\
112(3), 117(1), 118(1), 118 \mathrm{a}(1), 119(1), 121 \mathrm{a}(1), \\
125(1), 127(1), 130(6), 131(2), 150(2), 151 \mathrm{~b}(1), \\
155(2), 164(1), 165(2), 167(1), 172(2), 195(1), \\
211(1)\end{array}$ & \\
\hline $\begin{array}{l}\text { Rugilus orbiculatus (PAYKULL, } \\
\text { 1789) }\end{array}$ & $\bigcirc$ & & $41(1), 77(1), 126(93)$ & Sm04 \\
\hline Rugilus similis (ERICHSON, 1839) & O & 0 & $\begin{array}{l}\text { 20(1), 41(2), 47(3), 81(1), 81a(1), 101(3), 126(1), } \\
183(1)\end{array}$ & $\mathrm{Sm} 04$ \\
\hline $\begin{array}{l}\text { Scopaeus bicolor BAUDI DI SELVE, } \\
1848\end{array}$ & O & & & E30 \\
\hline Scopaeus cameroni CoIfFAIt, 1968 & O & 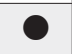 & 112(11), 121a(7), 130(9) & $\mathrm{C} 68^{44)}, \operatorname{Fr} 10$ \\
\hline $\begin{array}{l}\text { Scopaeus chalcodactylus (KoLE- } \\
\text { NATI, 1846) }\end{array}$ & O & O & $204 \mathrm{a}(3)$ & $\begin{array}{l}\text { Fr97, Fr07a, } \\
\text { Ko46 }\end{array}$ \\
\hline Scopaeus debilis Носннитн, 1851 & $\bigcirc$ & & $212(1)$ & Hh51, Fr12 \\
\hline Scopaeus gracilis (SPERK, 1835) & $\bigcirc$ & & 199(1) & Fr97, Fr07b \\
\hline $\begin{array}{l}\text { Scopaeus khnzoriani CoIfFAIT, } \\
1968\end{array}$ & $\mathrm{O}$ & & & C68, Fr09 \\
\hline $\begin{array}{l}\text { Scopaeus laevigatus (GYLLENHAL, } \\
\text { 1827) }\end{array}$ & O & & $92(1), 177(9), 212(1)$ & Fr10 \\
\hline $\begin{array}{l}\text { Scymbalium anale (NORDMANN, } \\
\text { 1837) }\end{array}$ & O & & & Sm04 \\
\hline $\begin{array}{l}\text { Sunius fulgocephalus (CoIfFAIT, } \\
\text { 1970) }\end{array}$ & $\mathrm{O}$ & & & A17g, C70d \\
\hline $\begin{array}{l}\text { Sunius khnzoriani (CoIfFAIT, } \\
\text { 1970) }\end{array}$ & O & O & $9(2), 12(1), 25(2), 26(22), 32(2), 76(1), 98(1)$ & A08c, A17g \\
\hline $\begin{array}{l}\text { Sunius melanocephalus (FABRI- } \\
\text { CIUs, 1792) }\end{array}$ & O & & & C70d ${ }^{43)}$ \\
\hline $\begin{array}{l}\text { Tetartopeus quadratus (PAYKULL, } \\
\text { 1789) }\end{array}$ & 0 & & $85 \mathrm{a}(1)$ & \\
\hline $\begin{array}{l}\text { Tetartopeus stylifer (REITTER, } \\
\text { 1909) }\end{array}$ & $\bigcirc$ & & & A17a \\
\hline \multicolumn{5}{|l|}{ St aphylininae } \\
\hline $\begin{array}{l}\text { Acylophorus glaberrimus (HERBST, } \\
\text { 1784) }\end{array}$ & O & & 190(4) & \\
\hline Astrapaeus ulmi (Rossi, 1790) & O & & & IK57a \\
\hline
\end{tabular}




\begin{tabular}{|c|c|c|c|c|}
\hline Species & AR & NK & Localities/Samples & References \\
\hline $\begin{array}{l}\text { Atanygnathus terminalis (ERICH- } \\
\text { SON, 1839) }\end{array}$ & 0 & & $\operatorname{ar}(1)$ & \\
\hline $\begin{array}{l}\text { Bisnius cephalotes (GRAVENHORST, } \\
\text { 1802) }\end{array}$ & O & & & $\begin{array}{l}\text { ATHW00, } \\
\text { IK64b }\end{array}$ \\
\hline $\begin{array}{l}\text { Bisnius fimetarius (GRAVENHORST, } \\
\text { 1802) }\end{array}$ & $\bigcirc$ & 0 & $\begin{array}{l}39(6), 67(6), 68(1), 68 \mathrm{a}(3), 80 \mathrm{~b}(11), 81(3), 81 \mathrm{a}(4) \\
90 \mathrm{a}(2), 105(13), 106(2), 108(1), 109(2), 110(12) \\
113(8), 114(44), 121(2), 126(3), 144(4)\end{array}$ & Sm04 \\
\hline $\begin{array}{l}\text { \#Bisnius microtophilus (CoIfFAIT, } \\
\text { 1967) }\end{array}$ & O & & & $\begin{array}{l}\text { ATHW00, } \\
\text { C67a }\end{array}$ \\
\hline $\begin{array}{l}\text { Bisnius pentheri (GANGLBAUER, } \\
\text { 1905) }\end{array}$ & $\bigcirc$ & & & $\begin{array}{l}\text { ATHW00, } \\
\text { C67a, IK64b }\end{array}$ \\
\hline Bisnius piochardi (FAUVEL, 1875) & O & & $122(1)$ & $\mathrm{C} 69^{48)}, \mathrm{Sr} 04$ \\
\hline Bisnius reitteri (EPPELSHEIM, 1889) & O & & $195 \mathrm{a}(3)$ & \\
\hline $\begin{array}{l}\text { Bisnius sordidus (GRAvENHORst, } \\
\text { 1802) }\end{array}$ & $\bigcirc$ & O & $\begin{array}{l}22(1), 70(2), 80 \mathrm{~b}(3), 81(1), 81 \mathrm{a}(1), 105(2), 107(3), \\
110(3), \quad 114(32), \quad 116(11), \quad 118 \mathrm{a}(1), \quad 122(315) \\
123(11), 124(2), 126(1)\end{array}$ & Sm04 \\
\hline $\begin{array}{l}{ }^{*} \text { Bisnius karkarensis SCHILLHAM- } \\
\text { MER, in prep. }{ }^{66)}\end{array}$ & 0 & & $67(1), 82(1), 86(9)$ & \\
\hline $\begin{array}{l}\text { Creophilus maxillosus maxillosus } \\
\text { (LinnAEus, 1758) }\end{array}$ & $\bigcirc$ & & & IK64b \\
\hline $\begin{array}{l}\text { Dinothenarus flavocephalus flavo- } \\
\text { cephalus (GoEZE, 1777) }\end{array}$ & $\bigcirc$ & 0 & 72(1), 112(1), 136(3), 148b(4), 148c(1), & Sz66b \\
\hline $\begin{array}{l}\text { Dinothenarus pubescens (DE GEER, } \\
\text { 1774) }\end{array}$ & ○ & & $86(1)$ & \\
\hline Emus hirtus (LINNAEUs, 1758) & O & & $44 \mathrm{~b}(2)$ & \\
\hline $\begin{array}{l}\text { Erichsonius cinerascens (GRAVEN- } \\
\text { HORST, 1802) }\end{array}$ & O & & $19(1), 20(2)$ & Pe80 \\
\hline Erichsonius dux SMEtana, 1967 & $\bigcirc$ & & & Pe80 \\
\hline $\begin{array}{l}\text { Erichsonius rivularis rivularis } \\
\text { (KIESENWETTER, 1858) }\end{array}$ & $\bigcirc$ & & $66 \mathrm{a}(1)$ & $\mathrm{C} 65^{21)}$ \\
\hline $\begin{array}{l}\text { Erichsonius subopacus (HocH- } \\
\text { HUTH, 1851) }\end{array}$ & $\bigcirc$ & O & $\begin{array}{l}36(2), 66 a(1), 100(2), 103(1), 175(1), 183(1), 185(1) \\
202(1)\end{array}$ & Br44 \\
\hline $\begin{array}{l}\text { \#Gabrius armeniacus CoIfFAIT, } \\
1966\end{array}$ & $\bigcirc$ & & & C66a, Sr03 \\
\hline Gabrius astutoides (STRAND, 1946) & ○ & O & 100(2), 113(1), 119(1), 125(1), 195(1), 199a(1) & \\
\hline $\begin{array}{l}\text { Gabrius femoralis (НосннUтн, } \\
\text { 1851) }\end{array}$ & 0 & 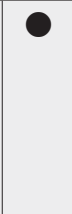 & $\begin{array}{l}12(3), 19(1), 22(4), 22 \mathrm{a}(1), 39(1), 50(2), 50 \mathrm{a}(1), \\
59(1), 73(1), 74(2), 77(1), 78(2), 79(1), 80(1), \\
80 \mathrm{~b}(2), 81(4), 81 \mathrm{a}(4), 82 \mathrm{a}(1), 83(1), 88(4), 89(6), \\
89 \mathrm{~b}(10), 90(1), 110(1), 112(2), 118(4), 118 \mathrm{a}(1), \\
119(1), 121(12), 121 \mathrm{a}(6), 126(31), 179(1)\end{array}$ & \\
\hline Gabrius latro Joy, 1913 & $\bigcirc$ & & $177(1), 190(1)$ & $\mathrm{C} 74^{22)}$ \\
\hline Gabrius muelleri GRIDELLI, 1928 & 0 & 0 & $\begin{array}{l}41(2), 46(1), 53(2), 66(1), 66 b(1), 88(1), 93(1), \\
98(4), 101(2), 103(1)\end{array}$ & \\
\hline $\begin{array}{l}\text { Gabrius nigritulus (GRAVENHORST, } \\
\text { 1802) }\end{array}$ & $\bigcirc$ & ○ & 100(11), 179(2), 192(2), 194(1), 201(1) & Sm04 \\
\hline Gabrius ravasinii GRIDELLI, 1920 & O & & $127(4), 204 a(1)$ & \\
\hline Gabrius robustus SMETANa, 1953 & 0 & & $\begin{array}{l}16(1), 67(2), 77(3), 82(2), 85 a(4), 85 c(11), 128(27), \\
128 \mathrm{a}(1)\end{array}$ & \\
\hline
\end{tabular}




\begin{tabular}{|c|c|c|c|c|}
\hline Species & AR & NK & Localities/Samples & References \\
\hline Gabrius sacerdotalis Joy, 1913 & $\bullet$ & & $66 \mathrm{a}(2), 73(3), 93 \mathrm{a}(1)$ & \\
\hline Gabrius subnigritulus Joy, 1913 & $\bullet$ & $\bullet$ & 103(4), 202(1) & \\
\hline Gabrius suffragani Joy, 1913 & 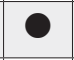 & & $180(2), 181(4)$ & \\
\hline Gabrius toxotes Joy, 1913 & 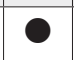 & & $179(1)$ & \\
\hline $\begin{array}{l}\text { Gabrius trossuliformis SCHILLHAM- } \\
\text { MER, } 1999\end{array}$ & $\bigcirc$ & & $\begin{array}{l}\text { 18(1), 21(15), 44(1), 45(9), 46(3), 49(1), 67(1), } \\
77(2), 82(2), 85 \mathrm{a}(4), 85 \mathrm{c}(3), 86(1), 94(7), 128(14)\end{array}$ & $\begin{array}{l}\text { ATHW00 } \\
\text { C66a }\end{array}$ \\
\hline $\begin{array}{l}\text { Gauropterus fulgidus (FABRICIUS, } \\
\text { 1787) }\end{array}$ & $\bigcirc$ & & & Sm04 \\
\hline $\begin{array}{l}\text { Gauropterus sanguinipennis } \\
\text { (KoLENATI, 1846) }\end{array}$ & $\bigcirc$ & & $178(1)$ & Ko46, Bo11b \\
\hline $\begin{array}{l}\text { Gyrohypnus angustatus STEPHENS, } \\
1833\end{array}$ & $\bigcirc$ & $\bullet$ & $\begin{array}{l}19(1), 20(2), 32(1), 33(2), 39(9), 41(3), 67(1), \\
80 \mathrm{~b}(1), 82(3), 103(1), 121 \mathrm{a}(4), 126(9), 130(1), \\
143(1), 179(2), 181(1), 186(1), 199 \mathrm{a}(1)\end{array}$ & Sm04 \\
\hline $\begin{array}{l}\text { Gyrohypnus fracticornis } \\
\text { (O. MüLLER, 1776) }\end{array}$ & $\bigcirc$ & $\bullet$ & $\begin{array}{l}22(1), 39(2), 44(1), 45(1), 54(1), 80 \mathrm{~b}(4), 86(2), \\
110(3), 114(58), 115(1), 116(4), 123(5), 139(1)\end{array}$ & $\mathrm{Sm} 04$ \\
\hline $\begin{array}{l}\text { Gyrohypnus punctulatus (PAYKULL, } \\
\text { 1789) }\end{array}$ & $\bullet$ & & $8(1), 80 \mathrm{~b}(2), 81 \mathrm{a}(1), 86(3)$ & \\
\hline $\begin{array}{l}\text { Heterothops balthasari SMETANA, } \\
1967\end{array}$ & O & & & Sm04 \\
\hline $\begin{array}{l}\text { Heterothops dissimilis (GRAVEN- } \\
\text { HORST, 1802) }\end{array}$ & $\bigcirc$ & & $\begin{array}{l}41(6), 43(1), 46(1), 47(3), 67(1), 76(1), 80 \mathrm{~b}(2), \\
92(3), 95(1), 126(1), 130(1)\end{array}$ & $\begin{array}{l}\text { ASpp, } \\
\text { ATHW00, C77 } \\
\text { 56), Mo60 }\end{array}$ \\
\hline $\begin{array}{l}\text { Heterothops laeticolor ReITteR, } \\
1891\end{array}$ & O & & & $\begin{array}{l}\text { ASpp, } \\
\text { ATHW00 }\end{array}$ \\
\hline $\begin{array}{l}\text { \#Heterothops macrops COIFFAIT, } \\
1977\end{array}$ & $\bigcirc$ & & & C77 \\
\hline $\begin{array}{l}\text { \#Heterothops microtophilus CoIF- } \\
\text { FAIT, } 1977\end{array}$ & $\bigcirc$ & & & $\begin{array}{l}\text { ASpp, } \\
\text { ATHW00, C77 }\end{array}$ \\
\hline $\begin{array}{l}\text { Heterothops praevius ERICHSON, } \\
1839\end{array}$ & $\bigcirc$ & $\bullet$ & $\begin{array}{l}\text { 22(35), 22a(5), 30(19), 31(6), 54(1), 57(5), 70(2), } \\
71(3), 72(2), 80 \mathrm{a}(3), 81 \mathrm{a}(2), 89(6), 89 \mathrm{~b}(7), 114(14), \\
116(1), 118(1), 122(3), 123(10)\end{array}$ & $\begin{array}{l}\text { ASpp, } \\
\text { ATHW00, } \\
\text { IK66 }\end{array}$ \\
\hline $\begin{array}{l}\text { Jurecekia asphaltina (ERICHSON, } \\
1840 \text { ) }\end{array}$ & $\bigcirc$ & & & ATHW00, Sr04 \\
\hline $\begin{array}{l}\text { \#Leptacinus armeniacus CoIfFAIT, } \\
1966\end{array}$ & $\bigcirc$ & & & A17f, C66b \\
\hline $\begin{array}{l}\text { Leptacinus batychrus (GYLLENHAL, } \\
\text { 1827) }\end{array}$ & 0 & & & E30 \\
\hline $\begin{array}{l}\text { Leptacinus nigerrimus COIFFAIT, } \\
1971\end{array}$ & $\bigcirc$ & & & Bo11a \\
\hline $\begin{array}{l}\text { Leptacinus pusillus (STEPHENS, } \\
1833 \text { ) }\end{array}$ & 0 & & & $\mathrm{Sm} 04$ \\
\hline $\begin{array}{l}\text { Leptacinus sulcifrons (STEPHENS, } \\
\text { 1833) }\end{array}$ & $\bigcirc$ & & $67(1), 86(1)$ & Sm04 \\
\hline $\begin{array}{l}\text { Neobisnius prolixus (ERICHSON, } \\
1840 \text { ) }\end{array}$ & $\bigcirc$ & & 8b(4), 14(1), 120(4), 185(4), 190(1), 199(4) & Hh49 \\
\hline $\begin{array}{l}\text { Nudobius lentus (GraVENHORST, } \\
\text { 1806) }\end{array}$ & $\bullet$ & & $136(1)$ & \\
\hline $\begin{array}{l}\text { Nudobius umbratus (Мот- } \\
\text { sCHULSKY, 1860) }\end{array}$ & $\bigcirc$ & & & $\begin{array}{l}\text { ATHW00, } \\
\text { IK57a }\end{array}$ \\
\hline
\end{tabular}




\begin{tabular}{|c|c|c|c|c|}
\hline Species & AR & NK & Localities/Samples & References \\
\hline $\begin{array}{l}\text { Ocypus curtipennis (MoT- } \\
\text { sCHULSKY, 1849) }\end{array}$ & O & & $36(1), 185(1)$ & E30 \\
\hline $\begin{array}{l}\text { Ocypus forficularius (MоT- } \\
\text { SCHULSKY, 1860) }\end{array}$ & 0 & & $6(1), 7(4), 135(1)$ & \\
\hline $\begin{array}{l}\text { Ocypus fulvipennis ERICHSON, } \\
1840\end{array}$ & 0 & & $\begin{array}{l}\text { 9(1), 40(1), 41(1), 74(1), 88(1), 126(3), 140(1), } \\
179(1)\end{array}$ & \\
\hline $\begin{array}{l}\text { Ocypus hochhuthi EPPELSHEIM, } \\
1878\end{array}$ & 0 & & $22(1), 156(2)$ & \\
\hline Ocypus mus (BRULlÉ, 1832) & O & & $26(7), 35(1)$ & \\
\hline $\begin{array}{l}\text { Ocypus nitens nitens (SCHRANK, } \\
\text { 1781) }\end{array}$ & O & & & $\mathrm{Sm} 04$ \\
\hline $\begin{array}{l}\text { Ocypus ophthalmicus ophthalmicus } \\
\text { (ScOPOLI, 1763) }\end{array}$ & $\mathrm{O}$ & & & Hh49 ${ }^{25)}$ \\
\hline $\begin{array}{l}\text { Ocypus orientis SMETANA \& } \\
\text { DAVIES, } 2000\end{array}$ & O & & & $\mathrm{E} 30^{26)}$ \\
\hline $\begin{array}{l}\text { Ocypus picipennis caucasicus } \\
\text { (MüLLER, 1926) }\end{array}$ & O & 0 & $\begin{array}{l}\text { 26(4), 65(9), 81b(1), 115(1), 116(1), 139(2), 183(4), } \\
185(1), 193(2), 202(1), 204 \mathrm{a}(1)\end{array}$ & H01 \\
\hline $\begin{array}{l}\text { Ocypus sericeicollis (MÉNÉTRIÉs, } \\
\text { 1832) }\end{array}$ & $\mathrm{O}$ & O & $22(1), 88(5), 89 b(1), 114(2), 116(1)$ & $\mathrm{Sm} 04$ \\
\hline $\begin{array}{l}\text { Ocypus syriacus primigenius } \\
\text { (MüLLER, 1923) }\end{array}$ & O & & & Mü23 \\
\hline $\begin{array}{l}{ }^{*} \text { Ontholestes chalcopygus (HocH- } \\
\text { HUTH, 1849) }\end{array}$ & O & & & C74 \\
\hline $\begin{array}{l}\text { Ontholestes murinus (LINNAEUS, } \\
\text { 1758) }\end{array}$ & $\bigcirc$ & O & $105(1), 180(1)$ & Hh49 \\
\hline Othius grandis Носннuтн, 1849 & $\mathrm{O}$ & 0 & $58(1), 72(1), 114(3)$ & A03 \\
\hline $\begin{array}{l}\text { Othius lapidicola Märkel \& } \\
\text { KIESENWETTER, } 1848\end{array}$ & O & & $\begin{array}{l}2(4), 4(16), 5(1), 6(6), 7(6), 11(20), 11 \mathrm{a}(20), 11 \mathrm{~b}(33), \\
30(11), 31(4), 33(4), 51(8), 55(3), 58(4), 59(1), \\
60(1), 62(1), 63(12), 69(4), 68 \mathrm{a}(1), 69(4), 70(2), \\
71(1), 72(1), 74(1), 75(10), 81(1), 82 \mathrm{a}(1), 88(21), \\
89(6), 89 \mathrm{~b}(2), 90 \mathrm{a}(1), 91(1), 92(4), 97(1), 118(20), \\
119(4), 124(4), 125(5), 126(4)\end{array}$ & A97, A03, A15 \\
\hline Othius punctulatus (GoEzE, 1777) & O & O & $\begin{array}{l}1(1), 2(2), 3(3), 4(5), 6(1), 7(3), 11(8), 11 a(3), \\
11 b(4), 12(2), 51(2), 55(1), 62(1), 69(3), 75(5), \\
95(1), 96(1), 110(1), 135(1), 148 b(1)\end{array}$ & A97, A03 \\
\hline $\begin{array}{l}\text { Othius stenocephalus EPPELSHEIM, } \\
1881\end{array}$ & O & & $6(2), 7(2), 59(2), 68(1), 69(2)$ & \\
\hline $\begin{array}{l}\text { Phacophallus parumpunctatus } \\
\text { (GYLLENHAL, 1827) }\end{array}$ & O & & & Sm04 \\
\hline $\begin{array}{l}\text { Philonthus aculeatus CoIfFAIT, } \\
1963\end{array}$ & O & 0 & $81 \mathrm{a}(2), 110(2), 112(3), 121 \mathrm{a}(2), 126(2), 127(1)$ & Am00 \\
\hline $\begin{array}{l}\text { Philonthus alberti SCHILLHAMMER, } \\
2000\end{array}$ & O & ○ & $\begin{array}{l}36(1), 68(1), 90(1), 103(4), 105(2), 110(7), 112(9), \\
113(2), 114(2), 125(1), 127(3), 195 \mathrm{a}(1)\end{array}$ & Sm04 \\
\hline $\begin{array}{l}\text { Philonthus alpinus EPPELSHEIM, } \\
1875\end{array}$ & O & & $44(59)$ & \\
\hline $\begin{array}{l}\text { Philonthus atratus (GRAVENHORST, } \\
\text { 1802) }\end{array}$ & O & & $6(1), 76(1), 120(6), 179(2)$ & E30 \\
\hline
\end{tabular}




\begin{tabular}{|c|c|c|c|c|}
\hline Species & AR & NK & Localities/Samples & References \\
\hline $\begin{array}{l}\text { Philonthus carbonarius (GRAVEN- } \\
\text { HORST, 1802) }\end{array}$ & 0 & O & $\begin{array}{l}14(1), 29(1), 39(1), 70(1), 88(1), 89 b(1), 95(1), \\
106(1), 108(1), 114(1), 116(1), 127(1), 128(1), \\
147(4), 156(4)\end{array}$ & \\
\hline $\begin{array}{l}\text { Philonthus caucasicus NordmanN, } \\
1837\end{array}$ & O & & & Sm04 \\
\hline $\begin{array}{l}\text { Philonthus cochleatus SCHEER- } \\
\text { PELTZ, } 1937\end{array}$ & O & & & $\mathrm{C} 74$ \\
\hline $\begin{array}{l}\text { Philonthus cognatus STEPHENS, } \\
1832\end{array}$ & 0 & 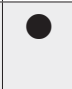 & $\begin{array}{l}\text { 75(1), 89(2), 110(1), 114(4), 115(1), 116(1), 120(1), } \\
126(1), 130(1), 183(1)\end{array}$ & \\
\hline $\begin{array}{l}\text { Philonthus concinnus (GRAVEN- } \\
\text { HORST, 1802) }\end{array}$ & O & 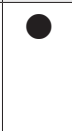 & $\begin{array}{l}14(3), 17(1), 21(2), 22 \mathrm{a}(1), 36(10), 80 \mathrm{~b}(4), 81(2), \\
81 \mathrm{a}(3), 85(1), 85 \mathrm{c}(1), 90 \mathrm{a}(1), 94(1), 99(1), 109(1) \\
114(3), 120(2), 121 \mathrm{a}(1), 123(4), 126(9), 128(2)\end{array}$ & $\mathrm{Sm} 04$ \\
\hline $\begin{array}{l}\text { Philonthus corruscus (GRAVEN- } \\
\text { HORST, 1802) }\end{array}$ & O & & $76(1), 157(1)$ & IK64b \\
\hline $\begin{array}{l}\text { Philonthus cruentatus (GMELIN, } \\
\text { 1790) }\end{array}$ & $\bigcirc$ & ○ & $44(12), 107(1), 122(1), 126(1), 179(1)$ & H01 \\
\hline $\begin{array}{l}\text { Philonthus debilis (GRAVENHORST, } \\
\text { 1802) }\end{array}$ & O & & & $\mathrm{Sm} 04$ \\
\hline $\begin{array}{l}\text { Philonthus dimidiatipennis ERICH- } \\
\text { sON, } 1840\end{array}$ & O & & & Hh49 \\
\hline $\begin{array}{l}\text { Philonthus discoideus (GRAVEN- } \\
\text { HORST, 1802) }\end{array}$ & O & & & Sm04 \\
\hline $\begin{array}{l}\text { Philonthus ebeninus (GRAVEN- } \\
\text { HORST, 1802) }\end{array}$ & O & O & $44(1), 78(1), 81(1), 116(1)$ & $\mathrm{Sm} 04$ \\
\hline $\begin{array}{l}\text { Philonthus eriwanensis BERN- } \\
\text { HAUER \& SCHUBERT, } 1914\end{array}$ & O & & & ATHW00, C74 \\
\hline $\begin{array}{l}\text { Philonthus fumarius (GRAVEN- } \\
\text { HORST, 1806) }\end{array}$ & 0 & & $66 a(1)$ & \\
\hline $\begin{array}{l}\text { Philonthus intermedius (LACOR- } \\
\text { DAIRE, 1835) }\end{array}$ & O & 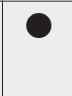 & $82(1), 115(1)$ & $\mathrm{Sm} 04$ \\
\hline Philonthus juvenilis PEYron, 1858 & 0 & & $185(1)$ & \\
\hline $\begin{array}{l}\text { Philonthus laminatus (CREUTzER, } \\
\text { 1799) }\end{array}$ & $\bigcirc$ & 0 & 107(1), 114(1), 115(1), 116(1), 122(1), 126(1) & Sm04 \\
\hline Philonthus laxatus FAUveL, 1875 & O & & & Hh62 ${ }^{27)}$ \\
\hline $\begin{array}{l}\text { Philonthus lindbergi SCHEERPELTZ, } \\
1958\end{array}$ & ○ & & 183(1), 201(1) & \\
\hline $\begin{array}{l}\text { Philonthus longicornis STEPHENS, } \\
1832\end{array}$ & O & & & $\mathrm{Sm} 04$ \\
\hline $\begin{array}{l}\text { Philonthus nigrita (GRAVENHORST, } \\
\text { 1806) }\end{array}$ & O & & 201(1) & \\
\hline $\begin{array}{l}\text { Philonthus nitidicollis (LACOR- } \\
\text { DAIRE, 1835) }\end{array}$ & O & & $21 \mathrm{a}(1), 86(1), 94 \mathrm{a}(1), 139(3)$ & Sm04 \\
\hline $\begin{array}{l}\text { Philonthus parvicornis (GRAVEN- } \\
\text { HORST, 1802) }\end{array}$ & O & & $44(7), 177(1)$ & $\mathrm{Sm} 04$ \\
\hline $\begin{array}{l}\text { Philonthus picimanus (MÉNÉTRIÉs, } \\
\text { 1832) }\end{array}$ & $\bigcirc$ & & $\begin{array}{l}33(4), 39(1), 46(1), 49(2), 53(1), 66(1), 67(10), \\
90(1), 94(1), 118(1), 126(8), 127(1), 135(4), 156(1), \\
179(2), 181(1)\end{array}$ & Sm04 \\
\hline $\begin{array}{l}\text { Philonthus politus (LINNAEUs, } \\
\text { 1758) }\end{array}$ & O & & $148 \mathrm{a}(1), 148 \mathrm{~b}(1), 149 \mathrm{~b}(1), 179(1)$ & Sm04 \\
\hline
\end{tabular}




\begin{tabular}{|c|c|c|c|c|}
\hline Species & AR & NK & Localities/Samples & References \\
\hline $\begin{array}{l}\text { Philonthus punctus punctus } \\
\text { (GRAVENHORST, 1802) }\end{array}$ & O & & & Sm04 \\
\hline $\begin{array}{l}\text { Philonthus quisquiliarius quisquili- } \\
\text { arius (GylleNHAL, 1810) }\end{array}$ & 0 & & 183(3), 190(3), 201(4), 203a(2), a(38) & Sm04 \\
\hline Philonthus rectangulus SHARP, 1874 & 0 & & $86(1)$ & ATHW00 \\
\hline $\begin{array}{l}\text { Philonthus rubripennis STEPHENS, } \\
1832\end{array}$ & O & $\bullet$ & $\begin{array}{l}36 \mathrm{a}(1), 100(1), 120(2), 179(5), 185(3), 199 \mathrm{~b}(1), \\
204 \mathrm{a}(1)\end{array}$ & Sm04 \\
\hline $\begin{array}{l}\text { Philonthus rufimanus ERICHSON, } \\
1840\end{array}$ & 0 & & $8 \mathrm{~b}(11), 14(6), 120(13)$ & \\
\hline $\begin{array}{l}\text { Philonthus rufimargo ReITTER, } \\
1909\end{array}$ & O & & & R09 \\
\hline $\begin{array}{l}\text { Philonthus sanguinolentus (GRA- } \\
\text { vENHORST, 1802) }\end{array}$ & O & & & C74 \\
\hline $\begin{array}{l}\text { Philonthus spinipes kabardensis } \\
\text { (Bolov \& KRYZHANOvSKIJ, 1969) }\end{array}$ & 0 & & & H01 \\
\hline $\begin{array}{l}\text { Philonthus splendens (FABRICIUS, } \\
\text { 1792) }\end{array}$ & 0 & & $46(1)$ & \\
\hline $\begin{array}{l}\text { Philonthus succicola THомson, } \\
1860\end{array}$ & O & & $148 \mathrm{~b}(3)$ & Sm04 \\
\hline $\begin{array}{l}\text { Philonthus svanetiensis COIFFAIT, } \\
1974\end{array}$ & 0 & & $4(2), 45(22), 45 a(1), 46(14), 49 a(2)$ & \\
\hline $\begin{array}{l}\text { Philonthus tenuicornis MULSANT \& } \\
\text { REY, } 1853\end{array}$ & O & $\bullet$ & $\begin{array}{l}\text { 41(1), 44(3), 62(5), 70(1), 75(2), 86(2), 103(2), } \\
\text { 133(3), 135(1), 136(4), 183(1) }\end{array}$ & Sm04 \\
\hline $\begin{array}{l}\text { Philonthus varians (PAYKULL, } \\
\text { 1789) }\end{array}$ & O & & $7(1), 15(1), 23 a(1)$ & IK64b \\
\hline $\begin{array}{l}\text { Philonthus velatipennis Solsky, } \\
1869\end{array}$ & O & & $\mathrm{a}(11)$ & Pe80 \\
\hline $\begin{array}{l}\text { Philonthus ventralis (GRAVEN- } \\
\text { HORST, 1802) }\end{array}$ & 0 & & & Sm04 \\
\hline $\begin{array}{l}\text { Philonthus viridipennis FAUveL, } \\
1875\end{array}$ & O & & & $\mathrm{Br} 01^{28)}$ \\
\hline Physetops tataricus (Pallas, 1773) & O & & & $\begin{array}{l}\text { ATHW00, } \\
\text { IK64b }^{54}, \text { SG05 }\end{array}$ \\
\hline $\begin{array}{l}\begin{array}{l}\text { Platydracus chalcocephalus (FABRI- } \\
\text { cIUs, 1801) }\end{array} \\
\end{array}$ & 0 & 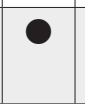 & $\begin{array}{l}8(1), \quad 106(1), \quad 112(2), \quad 127(1), \quad 148 \mathrm{~b}(1), \quad 151 \mathrm{a}(2), \\
156(2), 157(8), 199 \mathrm{a}(2)\end{array}$ & \\
\hline $\begin{array}{l}\text { Platydracus flavopunctatus } \\
\text { (LATREILle, 1804) }\end{array}$ & 0 & & $120(1)$ & Hh49 ${ }^{29)}$ \\
\hline $\begin{array}{l}\text { Platydracus meridionalis (RosEN- } \\
\text { HAUER, 1847) }\end{array}$ & O & & & Fa74 \\
\hline $\begin{array}{l}\text { Platydracus stercorarius stercora- } \\
\text { rius (OLIVIER, 1795) }\end{array}$ & O & & & Sm04 \\
\hline $\begin{array}{l}\text { Platyprosopus bagdadensis STIER- } \\
\text { LIN, } 1867\end{array}$ & 0 & & & Sm04 \\
\hline $\begin{array}{l}\text { Platyprosopus elongatus MANNER- } \\
\text { HEIM, } 1830\end{array}$ & O & & & Mm30 \\
\hline $\begin{array}{l}\text { Quedius acuminatus НосннUтн, } \\
1849\end{array}$ & O & & & H01 \\
\hline
\end{tabular}




\begin{tabular}{|c|c|c|c|c|}
\hline Species & AR & NK & Localities/Samples & References \\
\hline $\begin{array}{l}\text { Quedius boops (GRAVENHORST, } \\
\text { 1802) }\end{array}$ & 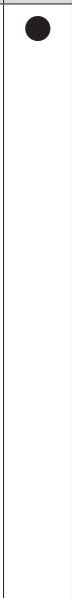 & 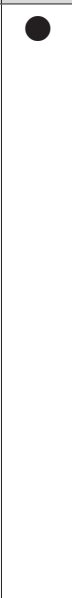 & $\begin{array}{l}7(1), 9(24), 11(1), 11 \mathrm{a}(2), 11 \mathrm{~b}(3), 12(1), 13(31), \\
15(2), 19(18), 20(2), 22(25), 22 \mathrm{a}(16), 23(1), 30(23), \\
31(2), 32(1), 33(1), 36(4), 39(43), 40(6), 41(2), \\
46(4), 47(2), 48(2), 49 \mathrm{a}(1), 50(19), 50 \mathrm{a}(1), 51(1), \\
54(3), 55(1), 57(1), 59(1), 60(5), 61(1), 63(1), 64(3), \\
67(3), 68(3), 69(2), 70(14), 71(2), 72(1), 73(2), \\
74(6), 75(9), 76(24), 77(6), 79(1), 81(34), 81 \mathrm{a}(20), \\
82(27), 82 \mathrm{a}(26), 83(17), 85 \mathrm{a}(1), 88(46), 89(142), \\
89 \mathrm{a}(3), 89 \mathrm{~b}(221), 90 \mathrm{a}(2), 91(10), 92(3), 93(3), \\
95(29), 100(2), 104(1), 105(1), 107(7), 108(20), \\
109(4), 110(65), 112(4), 113(1), 114(38), 115(2), \\
116(42), 118(9), 118 \mathrm{a}(7), 119(8), 121(13), 121 \mathrm{a}(1), \\
123(2), 124(3), 126(258), 127(1), 128(1), 129(2), \\
131(30), 135(5), 184(1)\end{array}$ & \\
\hline Quedius brevalatus Assing, 2017 & O & & $88(21)$ & A17e \\
\hline $\begin{array}{l}\text { Quedius brevicornis (THOMson, } \\
1860 \text { ) }\end{array}$ & O & & $151 \mathrm{a}(1)$ & \\
\hline Quedius cinctus (PAYKULL, 1790) & $\bigcirc$ & O & $\begin{array}{l}22(6), 55(1), 68(2), 80 \mathrm{~b}(1), 81 \mathrm{a}(1), 82(1), 114(12) \\
118(2), 123(3)\end{array}$ & Sm04 \\
\hline $\begin{array}{l}\text { Quedius cohaesus EPPELSHEIM, } \\
1888\end{array}$ & 0 & O & $\begin{array}{l}22(39), 30(34), 31(1), 32(37), 33(4), 36(1), 39(19), \\
40(4), 68(2), 81(15), 81 \mathrm{a}(7), 82(2), 89(4), 89 \mathrm{~b}(30), \\
90(8), 90 \mathrm{a}(18), 91(14), 100(11), 101(2), 101 \mathrm{a}(1), \\
105(3), 107(10), 108(3), 109(16), 110(14), 112(109), \\
113(12), 114(210), 115(75), 116(49), 117(4), \\
121(21), 121 \mathrm{a}(52), 123(1), 125(9), 126(50), 127(19), \\
130(1), 131(3), 145 \mathrm{a}(1)\end{array}$ & \\
\hline Quedius cruentus (OLIVIER, 1795) & $\bigcirc$ & & $132(4), 134(2)$ & A19b, IK61 ${ }^{61)}$ \\
\hline $\begin{array}{l}\text { Quedius curtipennis BERNHAUER, } \\
1908\end{array}$ & O & 0 & $\begin{array}{l}\text { 66(1), 69(1), 101(1), 109(2), 115(1), 130(3), 149(1), } \\
156(1)\end{array}$ & $\mathrm{Sm} 04$ \\
\hline Quedius edmundi CoIffart, 1969 & 0 & & $68 \mathrm{a}(1)$ & \\
\hline $\begin{array}{l}\text { Quedius fuliginosus (GRAVEN- } \\
\text { HORST, 1802) }\end{array}$ & 0 & 0 & $\begin{array}{l}\text { 2(1), 3(1), 4(1), 7(2), 19(3), 20a(1), 55(3), 62(1), } \\
\text { 63(1), 66b(1), 69(1), 72(1), 73(1), 75(1), 78(1), } \\
81(1), 89(1), 90(1), 105(1), 106(1), 107(1)\end{array}$ & \\
\hline $\begin{array}{l}\text { Quedius gridellii SCHEERPELTZ, } \\
1933\end{array}$ & O & & $30(1), 31(2), 39(1)$ & \\
\hline Quedius humeralis STEPHENS, 1832 & $\bigcirc$ & & & E30 \\
\hline Quedius invreae GRIDELLI, 1924 & 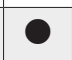 & & $155(1), 157(3)$ & \\
\hline Quedius korgeanus FAGEL, 1968 & $\bigcirc$ & & $40(1), 49(2), 123(4), 124(2), 125(1)$ & So04 \\
\hline \#Quedius latus Носннштн, 1851 & $\bigcirc$ & & & Hh51 \\
\hline Quedius levicollis (BRULLÉ, 1832) & 0 & 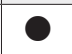 & $114(1), 136(3)$ & \\
\hline
\end{tabular}




\begin{tabular}{|c|c|c|c|c|}
\hline Species & AR & NK & Localities/Samples & References \\
\hline Quedius limbatus (HEER, 1839) & O & 0 & $\begin{array}{l}3(1), 3 a(1), 4(4), 6(3), 7(3), 9(20), 9 a(3), 10(1), \\
11(28), 11 a(20), 11 b(16), 12(7), 13(6), 15(5), 16(6), \\
19(2), 20 a(1), 21(5), 21 a(2), 22(2), 28(2), 30(12), \\
31(7), 32(23), 39(47), 40(1), 43(1), 46(3), 49 a(5), \\
50(45), 50 a(6), 52(1), 53(14), 58(2), 63(1), 64(3), \\
66(4), 66 a(8), 66 b(1), 67(19), 69(1), 70(1), 71(2), \\
72(12), 74(2), 75(3), 76(23), 77(2), 82(1), 83(2), \\
84(1), 85(1), 85 a(22), 85 \mathrm{c}(6), 87(1), 88(8), 89(4), \\
89 b(3), 91(4), 92(4), 93(2), 97(2), 98(8), 100(2), \\
102(1), 103(5), 104(1), 105(5), 106(1), 108(1), \\
112(29), 113(1), 115(1), 116(2), 118(16), 118 a(3), \\
119(6), 121(9), 121 a(19), 124(3), 125(2), 126(354), \\
127(5), 129(1), 131(2), 135(4), 136(6) ; 145(2), \\
148(3), 149 a(1)\end{array}$ & So02 \\
\hline $\begin{array}{l}\text { Quedius nitipennis (STEPHENS, } \\
\text { 1833) }\end{array}$ & O & & $\begin{array}{l}20(1), 23(1), 41(1), 67(30), 73(3), 76(1), 77(3), \\
78(1), 89(1)\end{array}$ & $\mathrm{C}_{67 \mathrm{~b}^{46)}}{ }^{4} \mathrm{So} 04$ \\
\hline $\begin{array}{l}\text { Quedius ochripennis (MÉNÉTRIÉs, } \\
\text { 1832) }\end{array}$ & $\bigcirc$ & O & 22(8), 88(1), 114(25), 116(1),135(3) & IK64b \\
\hline Quedius orientalis KoRGE, 1971 & & O & $112(5)$ & \\
\hline $\begin{array}{l}\text { Quedius scintillans(GRAVENHORST, } \\
\text { 1806) }\end{array}$ & & O & $114(22)$ & \\
\hline $\begin{array}{l}\text { Quedius semiobscurus (MARSHAM, } \\
\text { 1802) }\end{array}$ & ○ & ○ & 22a(2), 78(2), 79(4), 88(2), 114(7), 116(1), 118(1) & \\
\hline $\begin{array}{l}\text { Quedius suramensis EPPELSHEIM, } \\
1880\end{array}$ & O & & & So02 \\
\hline $\begin{array}{l}\text { Quedius suturalis suturalis KIESEN- } \\
\text { WETTER, } 1845\end{array}$ & O & & & So02 \\
\hline $\begin{array}{l}\text { Quedius tetrapunctatus CoIfFAIT, } \\
1977\end{array}$ & O & & & C77, IK61 ${ }^{45)}$ \\
\hline $\begin{array}{l}{ }^{*} \text { Quedius transcaucasicus IABLO- } \\
\text { KOFF-KHNZORIAN, } 1961\end{array}$ & O & & & IK61 \\
\hline Quedius umbrinus ERICHSON, 1839 & O & 0 & $\begin{array}{l}1(3), 6(2), 9(4), 13(1), 19(2), 20(10), 33(4), 39(6), \\
41(4), 49(1), 54(3), 63(2), 67(9), 75(2), 76(2), 77(5), \\
78(1), 81(4), 81 a(5), 82(4), 89(2), 89 b(2), 90(2), \\
90 a(3), 91(5), 95(2), 97(4), 100(4), 101(2), 103(1), \\
105(1), 106(2), 107(3), 112(11), 114(1), 116(1), \\
117(3), 121(1), 121 a(5), 125(3), 126(29), 130(3)\end{array}$ & A18f, So02 \\
\hline Quedius vexans EpPeLSHEIM, 1881 & O & & & ATHW00 \\
\hline $\begin{array}{l}\text { Quedius vulneratus GEMMINGER \& } \\
\text { HAROLD, } 1868\end{array}$ & O & & $4(7)$ & So02 \\
\hline Rabigus abauriae (GRIDELLI, 1924) & O & & $27(1)$ & C74 \\
\hline Rabigus pullus (NoRDMANN, 1837) & $\bigcirc$ & & $126(3)$ & C74 \\
\hline $\begin{array}{l}\text { Staphylinus caesareus caesareus } \\
\text { (CEDERHJELM, 1798) }\end{array}$ & O & & 61(2), 141(1), 185(1), 186(1), 194(2), 195(1) & Hn65 \\
\hline $\begin{array}{l}\text { Stenistoderus cephalotes cephalotes } \\
\text { (KRAATZ, 1858) }\end{array}$ & O & & & A17f, C66b ${ }^{30)}$ \\
\hline $\begin{array}{l}\text { \#Stenistoderus nanus (IABLOKOFF- } \\
\text { KHNZORIAN, 1961) }\end{array}$ & O & & & IK61 \\
\hline $\begin{array}{l}\text { Tasgius eppelsheimianus (ЈАКОВ- } \\
\text { soN, 1909) }\end{array}$ & O & 0 & $4(1), 58(1), 80 \mathrm{~b}(1), 105(2), 136(2)$ & \\
\hline
\end{tabular}




\begin{tabular}{|c|c|c|c|c|}
\hline Species & AR & NK & Localities/Samples & References \\
\hline $\begin{array}{l}\text { Tasgius gracilicornis (НосннUтн, } \\
\text { 1849) }\end{array}$ & O & - & 103(1), 157(1), 185(1), 194(2) & AM00 31) \\
\hline $\begin{array}{l}\text { Tasgius limbifrons (НосннUтн, } \\
\text { 1849) }\end{array}$ & O & & & Hh49 \\
\hline $\begin{array}{l}\text { Tasgius pedator pedator (GRAVEN- } \\
\text { HORST, 1802) }\end{array}$ & O & & & $\mathrm{Sm} 04$ \\
\hline $\begin{array}{l}\text { Tasgius protensus (MÉNÉTRIÉs, } \\
1832 \text { ) }\end{array}$ & O & & & Sm04 \\
\hline $\begin{array}{l}\text { *Xantholinus adustus Ushakov, } \\
1986\end{array}$ & O & & 126(1) & Uv86 \\
\hline $\begin{array}{l}\text { Xantholinus araxis ReITTER, } \\
1898^{32)}\end{array}$ & O & & $26(3)$ & A17f \\
\hline $\begin{array}{l}\text { Xantholinus audrasi CoIfFAIT, } \\
1956\end{array}$ & O & $\bullet$ & $\begin{array}{l}26(1), 35(1), 36(2), 64(1), 66 a(1), 73(1), 78(3), \\
79(4), 88(4), 89 \mathrm{~b}(1), 91(1), 100(1), 103(1), 112(3), \\
113(1), 114(1), 121 \mathrm{a}(1), 125(1), 126(10), 179(1), \\
183(1), 187(1), 203 \mathrm{a}(2)\end{array}$ & $\begin{array}{l}\text { A17f, ATHW00 } \\
\text { 33), Bo17, C56 } \\
\text { C70b }\end{array}$ \\
\hline $\begin{array}{l}\text { Xantholinus fortepunctatus Moт- } \\
\text { sCHULsкY, } 1860\end{array}$ & O & & $130(3)$ & A17f, R08 \\
\hline $\begin{array}{l}\text { Xantholinus haematodes KoLE- } \\
\text { NATI, } 1846\end{array}$ & & 0 & & Ko46 \\
\hline $\begin{array}{l}\text { Xantholinus khnzoriani COIFFAIT, } \\
1966\end{array}$ & O & & $7(1), 7 \mathrm{a}(3), 55(3), 68(2), 199 \mathrm{a}(2)$ & A17f, C66b \\
\hline $\begin{array}{l}\text { *Xantholinus kirschenblati BoR- } \\
\text { DONI, } 1975\end{array}$ & O & $\bullet$ & $\begin{array}{l}\text { 6(2), 12(1), 22(1), 26(1), 30(2), 32(2), 33(1), 43(2), } \\
47(1), 51(1), 52(2), 53(2), 63(2), 64(2), 66(1), \\
\text { 66a(1), 68(1), 75(1), 82(1), 82a(2), 83(1), 105(1), } \\
112(1), 124(1), 133(1), 135(2),\end{array}$ & A17f, Bo75 \\
\hline $\begin{array}{l}\text { Zeteotomus scripticollis (НосH- } \\
\text { HUTH, 1849) }\end{array}$ & 0 & & & $\begin{array}{l}\text { ATHW00, } \\
\text { IK57a }\end{array}$ \\
\hline
\end{tabular}

\section{Localities/samples:}

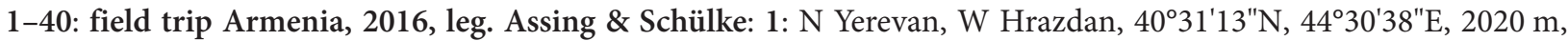
grassy slope with scattered oak, litter and roots sifted, 25.VI.2016; 2: N Yerevan, W Hrazdan, 40 $32^{\prime} 02^{\prime \prime} \mathrm{N}, 44^{\circ} 33^{\prime} 16^{\prime \prime} \mathrm{E}$ $2130 \mathrm{~m}$, secondary forest margin, litter and roots sifted, 25.VI.2016; 3: N Yerevan, W Hrazdan, 40 $30^{\prime} 28^{\prime \prime} \mathrm{N}, 44^{\circ} 34^{\prime} 12^{\prime \prime} \mathrm{E}$, $1870 \mathrm{~m}$, mixed deciduous forest margin, litter sifted, 25.VI.2016; 3a: same data as 3, but roots of grass and herbs sifted; 4: N Yerevan, NW Hrazdan, $40^{\circ} 41^{\prime} 40^{\prime \prime} \mathrm{N}, 44^{\circ} 29^{\prime} 16^{\prime \prime} \mathrm{E}, 2500 \mathrm{~m}$, grassy W-slope with scattered Salix, litter and roots of grass sifted 26.VI.2016; 5: N Yerevan, NW Hrazdan, $40^{\circ} 40^{\prime} 07^{\prime \prime} \mathrm{N}, 44^{\circ} 28^{\prime} 22^{\prime \prime} \mathrm{E}, 2100 \mathrm{~m}$, grassy W-slope with bushes, litter and grass roots sifted 26.VI.2016; 5a: same data as 5, but W-slope near stream, swept from vegetation; 6: N Yerevan, NW Hrazdan, 40 $38^{\prime} 07^{\prime} \mathrm{N}, 44^{\circ} 30^{\prime} 05^{\prime} \mathrm{E}, 2010 \mathrm{~m}$, mixed deciduous forest, litter and grass roots sifted,

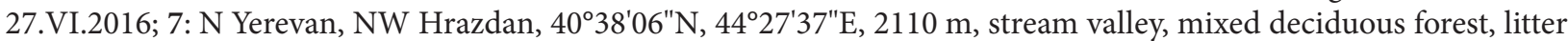
and grass roots sifted, 28.VI.2016; 7a: same data as 7, but moist margin of mountain track; 8: N Yerevan, NW Hrazdan, $40^{\circ} 33^{\prime} 45^{\prime \prime} \mathrm{N}, 44^{\circ} 23^{\prime} 41^{\prime} \mathrm{E}, 2000 \mathrm{~m}$, mixed deciduous forest margin, litter sifted, 28.VI.2016; 8a: same data as 8, but pasture, nests of Formica exsecta and Lasius flavus sifted; 8b: same data as 8, but stream bank with gravel; 9: S Martuni, Sulema Pass, $39^{\circ} 57^{\prime} 58^{\prime \prime} \mathrm{N}, 45^{\circ} 14^{\prime} 13^{\prime \prime} \mathrm{E}, 2340 \mathrm{~m}$, slope with small stream and scattered bushes, litter and roots near stream sifted, 29.VI.2016; 9a: same data as 9, but rocky slope with grass, moss, and fern, roots and moss sifted; 10: S Martuni, Sulema Pass, $39^{\circ} 58^{\prime} \mathrm{N}, 45^{\circ} 14^{\prime} \mathrm{E}, 2340 \mathrm{~m}$, road margin with bushes, litter and gravel sifted, 29.VI.2016; 11: ca. $50 \mathrm{~km}$ NW Sisian, Jermuk, $39^{\circ} 50^{\prime} 02^{\prime \prime} \mathrm{N}, 45^{\circ} 40^{\prime} 21^{\prime \prime} \mathrm{E}, 2110 \mathrm{~m}$, oak forest and forest margin, litter and roots sifted, 30.VI.2016; 11a: same data as 11, but 3.VII.2016; 11b: same data as 11, but 12.VII.2016; 12: $40 \mathrm{~km}$ NW Sisian, W-side of Vorotan Pass, 39 $42^{\prime} 36^{\prime \prime N}, 45^{\circ} 40^{\prime} 30^{\prime \prime} \mathrm{E} 1960 \mathrm{~m}$, dry oak forest, litter and roots sifted, 30.VI.2016; 13: $20 \mathrm{~km} \mathrm{~S}$ Sisian, Dastakert, $39^{\circ} 21^{\prime} 50^{\prime \prime} \mathrm{N}, 46^{\circ} 01^{\prime} 27^{\prime \prime} \mathrm{E}, 2080 \mathrm{~m}$, grassy slope with scattered bushes, litter and roots beneath bushes

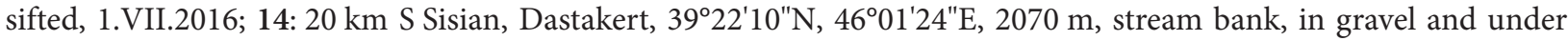
stones, 1.VII.2016; 15: $20 \mathrm{~km} \mathrm{SW}$ Sisian, 39 $25^{\prime} 09^{\prime \prime} \mathrm{N}, 45^{\circ} 55^{\prime} 09^{\prime \prime} \mathrm{E}, 1860 \mathrm{~m}$, grassy slope with bushes, litter and roots

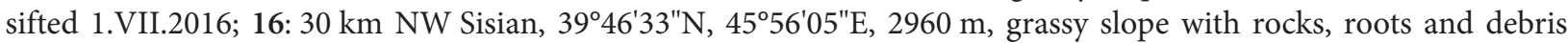


sifted, 2.VII.2016; 17: $30 \mathrm{~km}$ NW Sisian, 39 46'36"N, 45 56'03"E, $2970 \mathrm{~m}$, road margin and pasture, under stones, 2.VII.2016; 18: $30 \mathrm{~km} \mathrm{NW}$ Sisian, 39 $46^{\prime} 53^{\prime \prime} \mathrm{N}, 4^{\circ} 56^{\prime} 20^{\prime \prime} \mathrm{E}, 3000 \mathrm{~m}$, snowfields, under stones and debris sifted, 2.VII.2016; 19: $35 \mathrm{~km} \mathrm{NW}$ Sisian, 39 $40^{\prime} 59^{\prime \prime} \mathrm{N}, 45^{\circ} 46^{\prime} 50^{\prime \prime} \mathrm{E}, 2070 \mathrm{~m}$, stream valley, litter beneath bushes near stream sifted 3.VII.2016; 20: 35 km NW Sisian, 3941'01"N, 4546'46"E, 2080 m, swampy meadow, debris sifted, 3.VII.2016; 20a: same data, but litter and roots beneath bushes near small stream sifted; 21 : N Sisian, near Mt. Tsugh, 39² $41^{\prime} 10^{\prime \prime} \mathrm{N}$, $46^{\circ} 03^{\prime} 13^{\prime \prime E}, 3300 \mathrm{~m}$, soil and debris near snowfields sifted, 4.VII.2016; 21a: same data, but meadows, partly near snowfields, under stones; 22: $20 \mathrm{~km}$ SSE Goris, Shurnukh, 39²1'38"N, 46²4'33"E, $1720 \mathrm{~m}$, Quercus and Carpinus forest, litter and dead wood sifted, 5.VII.2016; 22a: same data as 22, but grassland near forest margin, litter beneath bushes sifted; 23: WSW Kapan, Meghri Pass, 3907'00"N, 4609'38"E, 2520 m, moist grassy slope with scattered bushes, litter and grass roots sifted, 6.VII.2016; 23a: same data as 23, but grassy slope, under stones; 23b: same data as 23, but grassy slope, in mushroom; 24: WSW Kapan, S Meghri Pass, 3905'56"N, 4609'47"E, 2090 m, stream valley, under stones, 6.VII.2016; 25: SW Kapan, 15 km N Meghri, 3901'44"N, 46²12'24"E, 1680 m, road margin, under stones, 6.VII.2016;

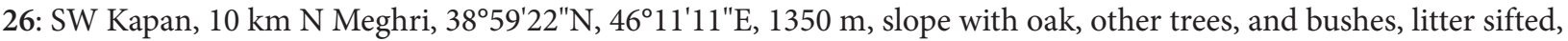
6.VII.2016; 27: SW Kapan, $10 \mathrm{~km}$ N Meghri, 38 $58^{\prime} 45^{\prime \prime} \mathrm{N}, 46^{\circ} 12^{\prime} 32^{\prime \prime} \mathrm{E}, 1130 \mathrm{~m}$, pasture near stream, under stones, 6.VII.2016; 28: WSW Kapan, S Meghri Pass, 3906'10"N, 46¹0'47"E. 2310 m, oak forest near tree line, oak litter sifted, 6.VII.2016; 28a: same data as 28, but road margin, in Formica nest; 29: 25 km S Kapan, Gomarants Pass, 3901'17"N, $46^{\circ} 22^{\prime} 01^{\prime \prime E}, 2230 \mathrm{~m}$, under stones, 7.VII.2016; 30: $25 \mathrm{~km}$ S Kapan, N Gomarants Pass 3901'32"N, 46²21'59"E, 2190 m,

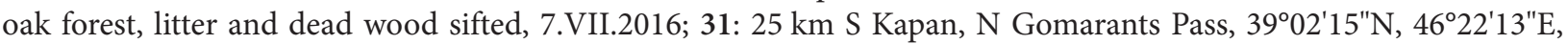
2050 m, oak forest with Acer, Carpinus, and fern undergrowth, litter and dead wood sifted, 7.VII.2016; 32: WSW Kapan, S Meghri Pass, 3905'20"N, 46 $10^{\prime} 35^{\prime \prime E}, 2170 \mathrm{~m}$, oak forest margin, litter (partly moist litter under bushes) sifted, 8.VII.2016; 33: WSW Kapan, S Meghri Pass, 3905'56"N, 4609'47"E, 2090 m, stream valley, litter near stream sifted, 8.VII.2016; 34: $10 \mathrm{~km}$ S Kapan, SE Chakaten, 3907'50"N, 46 $28^{\prime} 14^{\prime \prime} \mathrm{E}, 990 \mathrm{~m}$, mixed deciduous forest, litter and debris sifted, 9.VII.2016; 35: $15 \mathrm{~km}$ S Kapan, SE Chakaten, 3905'25"N, 46 $28^{\prime} 41^{\prime \prime E}, 1010 \mathrm{~m}$, mixed deciduous forest, litter between stones sifted, 9.VII.2016; 36: $20 \mathrm{~km}$ Kapan, W Tsav, 3903'04"N, 46 $26^{\prime} 12^{\prime \prime} \mathrm{E}, 1170 \mathrm{~m}$, stream valley, litter and flood debris near stream sifted, 9.VII.2016; 36a: same data as 36, but collected from gravel; 37: WSW Kapan, N Meghri

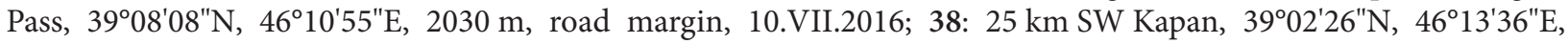
$1750 \mathrm{~m}$, margin of pista, under stone, 10.VII.2016; 39: $25 \mathrm{~km}$ SW Kapan, 3904'01"N , 46 $6^{\circ} 16^{\prime} 10^{\prime \prime} \mathrm{E}, 2150 \mathrm{~m}$, near stream, litter of Salix and debris sifted, 10.VII.2016; 40: $25 \mathrm{~km}$ SW Kapan, 3903'04"N, 46¹5'16"E, $1890 \mathrm{~m}$, stream valley, litter of Salix and other trees sifted, 10.VII.2016.

41-91: field trip Armenia, 2017, leg. Assing \& Schülke: 41: Gavar, 40²1'01"N, 4507'45"E, 1950 m, old poplar trees

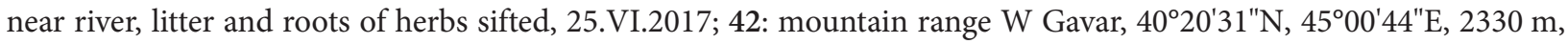

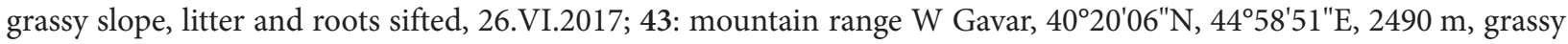
slope, litter and roots sifted, 26.VI.2017; 44: mountain range W Gavar, 40²0'N, 44 $57-58^{\prime} \mathrm{E}, 2600-2700 \mathrm{~m}$, horse dung sifted, 26.VI.2017; 44a: same data as 44, but under stones; 44b: same data as 44, but cow dung; 45: mountain range

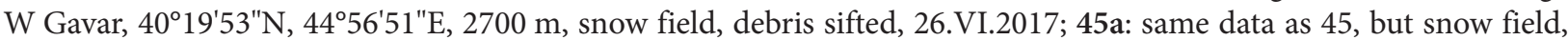
under stones; 46: mountain range W Gavar, 40 $19^{\prime} 59^{\prime \prime} \mathrm{N}, 44^{\circ} 56^{\prime} 53^{\prime \prime} \mathrm{E}, 2700 \mathrm{~m}$, debris near snow field and moist litter on

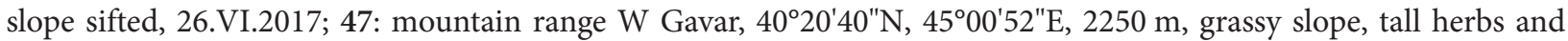

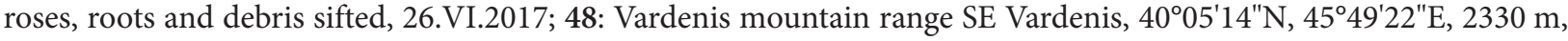
stream valley with Salix, litter and roots beneath Salix sifted, 27.VI.2017; 49: Vardenis mountain range SE Vardenis, $40^{\circ} 02^{\prime} 38^{\prime \prime} \mathrm{N}, 45^{\circ} 46^{\prime} 12^{\prime \prime} \mathrm{E}, 2730 \mathrm{~m}$, slope below snowfields, stream bank, debris sifted, 27.VI.2017; 49a: same data as 49, but grass and herb roots in shade of large rocks sifted; 50: Sevani mountain range E Sevan lake, Tsapatagh env., $40^{\circ} 23^{\prime} 51^{\prime \prime N}, 45^{\circ} 29^{\prime} 53^{\prime \prime E}, 2025 \mathrm{~m}$, oak forest margin, litter and roots sifted, 28.VI.2017; 50a: stream valley, litter and roots beneath bush at stream bank sifted; 51: SSE Dilijan, NW Semyonovka, 4040'09"N, 4452'35"E, 2050 m, Sorbus forest, litter sifted, 29.VI.2017; 52: SSE Dilijan, NW Semyonovka, 4040'12"N, 4453'05"E, $1900 \mathrm{~m}$, stream valley, forest margin

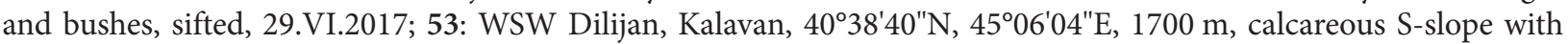
stream valley and oak forest, litter and roots sifted, 29.VI.2017; 54: NE Sevan Lake, Karmir pass, $40^{\circ} 33^{\prime} 48^{\prime \prime} \mathrm{N}, 45^{\circ} 18^{\prime} 01^{\prime \prime} \mathrm{E}$, $2150 \mathrm{~m}$, debris, grass, and roots beneath scattered Sorbus sifted, 30.VI.2017; 55: E Dilijan, road Ttujur-Berd, 40² $41^{\prime} 05^{\prime \prime} \mathrm{N}$, $45^{\circ} 20^{\prime} 18^{\prime \prime E}, 1900$ m, moist mixed deciduous forest with Fagus orientalis, Sorbus, etc., litter and roots sifted, 30.VI.2017; 56: E Dilijan, road Ttujur-Berd, 4041'47"N, 4519'23"E, 2040 m, litter near small stream sifted, 30.VI.2017; 57: E Dilijan, road Ttujur-Berd $40^{\circ} 44^{\prime} 04^{\prime \prime} \mathrm{N}, 45^{\circ} 18^{\prime} 10^{\prime E} \mathrm{E}, 1930 \mathrm{~m}$, slope with beech and bushes, litter, roots, and mushrooms sifted, 30.VI.2017; 58: road Berd-Ijevan, $40^{\circ} 52^{\prime} 26^{\prime \prime} \mathrm{N}, 45^{\circ} 18^{\prime} 00^{\prime \prime} \mathrm{E}, 1350 \mathrm{~m}$, beech forest margin, litter and roots sifted, 30.VI.2017; 59: road Dilijan-Vanadzor, $40^{\circ} 46^{\prime} 15^{\prime \prime} \mathrm{N}, 44^{\circ} 37^{\prime} 02^{\prime \prime} \mathrm{E}, 1790 \mathrm{~m}$, birch forest, litter sifted, 1.VII.2017;

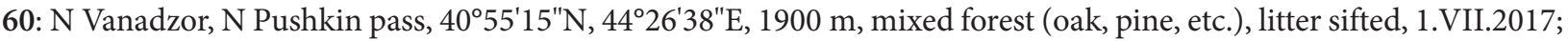
61: N Vanadzor, S Pushkin pass, $40^{\circ} 54^{\prime} 26^{\prime \prime} \mathrm{N}, 44^{\circ} 25^{\prime} 36^{\prime \prime} \mathrm{E}, 1880 \mathrm{~m}$, N-slope with scattered bushes, debris and grass roots sifted, 1.VII.2017; 62: N Vanadzor, S Pushkin pass, 4054'15"N, 44²5'46"E, $1850 \mathrm{~m}$, forest with Quercus, Fagus, Sorbus, etc., litter and roots sifted, 1.VII.2017; 63: N Vanadzor, S Pushkin pass, $40^{\circ} 54^{\prime} 00^{\prime \prime} \mathrm{N}, 44^{\circ} 25^{\prime} 55^{\prime \prime} \mathrm{E}, 1780 \mathrm{~m}$, forest margin with Quercus, Fagus, Sorbus, etc., litter and roots near small stream sifted, 1.VII.2017; 64: SW Gavar, 40 $15^{\prime} 18^{\prime \prime} \mathrm{N}$, $45^{\circ} 09^{\prime} 40^{\prime \prime} \mathrm{E}, 2170 \mathrm{~m}$, N-slope with scattered bushes, litter and roots roots sifted, 2.VII.2017; 65: SW Gavar, 40 $15^{\prime} 10^{\prime \prime} \mathrm{N}$, 
$45^{\circ} 09^{\prime} 25^{\prime \prime E}, 2180 \mathrm{~m}$, grassy N-slope near lake, under stones, 2.VII.2017; 66: ENE Dilijan, Hovk, 1290 m, 4047'39"N, $45^{\circ} 01^{\prime} 17 " \mathrm{E}$, stream valley, litter beneath Acer sifted, 3.VII.2017; 66a: same data as 66, but stream valley, moist litter near stream sifted; 66b: same data as 66, but forest (Quercus, Fagus, Acer, etc.) margin, litter and dead wood beneath old

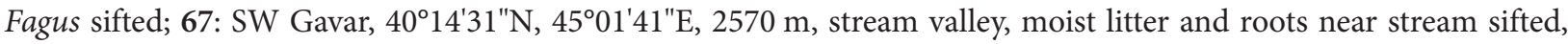
4.VII.2017; 68: WSW Dilijan, Kalavan, 40³7'52"N, 4505'46"E, 1960 m, forest with Quercus, Betula, and Carpinus, litter sifted, 5.VII.2017; 68a: same data as 68, but bark of dead oak sifted; 69: WSW Dilijan, Kalavan, $40^{\circ} 37^{\prime} 45^{\prime \prime} \mathrm{N}, 45^{\circ} 05^{\prime} 31^{\prime \prime} \mathrm{E}$, $2100 \mathrm{~m}$, forest at timber line with oak and birch, litter and large fungus on oak sifted, 5.VII.2017; 70: pass road E Ijevan, $40^{\circ} 52^{\prime} 00^{\prime \prime} \mathrm{N}, 45^{\circ} 13^{\prime} 19^{\prime \prime} \mathrm{E}, 1790 \mathrm{~m}$, forest with old Quercus and Carpinus, litter and roots sifted, 6.VII.2017; 71: pass road E Ijevan, $40^{\circ} 51^{\prime} 51^{\prime \prime} \mathrm{N}, 45^{\circ} 11^{\prime} 52^{\prime \prime} \mathrm{E}, 1630 \mathrm{~m}$, oak and beech forest, litter and roots sifted, 6.VII.2017; 72: pass road E Ijevan, $40^{\circ} 52^{\prime} 02^{\prime \prime} \mathrm{N}, 45^{\circ} 11^{\prime} 03^{\prime \prime E}, 1400 \mathrm{~m}$, young beech forest, litter and dead wood sifted, 6.VII.2017; 73: S Spitak, 4045'45"N, $44^{\circ} 16^{\prime} 23^{\prime \prime E}, 2000 \mathrm{~m}$, deforested stream valley, bank of small stream, roots and moss sifted, 7.VII.2017; 73a: same data as

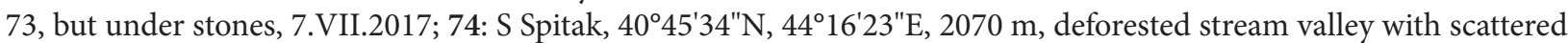

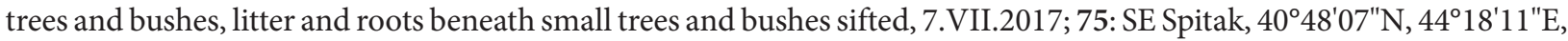

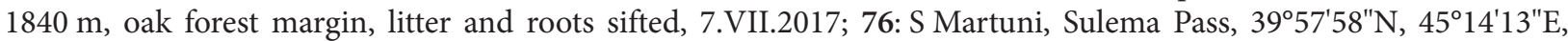
$2340 \mathrm{~m}$, slope with small stream and scattered bushes, litter and roots near stream sifted, 8.VII.2017; 77: $40 \mathrm{~km}$ NW Sisian, Vorotan Pass, 3941'48"N, 4541'28"E, 2090 m, grassy slope with scatteres bushes, litter, debris, and moss sifted, 8.VII.2017; 78: E Goris, Khndzoresk env., 39³1'32"N, 46²2'21"E, 1590 m, ruderal grassy slope with Rosa bushes and Rubus, litter and roots sifted, 9.VII.2017; 79: E Goris, Khndzoresk env., 39 30'58"N, 46 27'21"E, 1350 m, slope with dense small trees, litter and grass roots sifted, 9.VII.2017; 80: pass N Goris, 39³4'53"N, 46²19'39"E, $2110 \mathrm{~m}$, grassy slope with stones, under stones, 9.VII.2017; 80a: same data as 80, but mouse nest under stone sifted; 80b: same data as

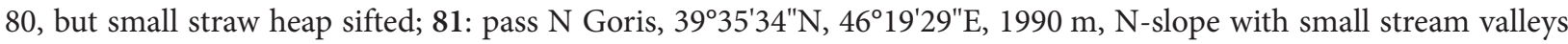
with and without water, litter, debris, and moss sifted, 9.VII.2017; 81a: same data as 81, but N-slope with small stream valleys with water, litter, debris, and moss sifted, 10.VII.2017; 81b: same data as 81a, but grassy N-slope, under stone; 82: $40 \mathrm{~km}$ NW Sisian, Vorotan Pass, 39 $40^{\prime} 33^{\prime \prime} \mathrm{N}, 45^{\circ} 45^{\prime} 07^{\prime \prime} \mathrm{E}, 2140 \mathrm{~m}$, stream valley with Salix, litter and roots sifted, 10.VII.2017; 82a: same data as 82, but stream valley, rose bushes, litter and roots sifted; 83: $40 \mathrm{~km}$ NW Sisian, Vorotan Pass, $39^{\circ} 41^{\prime} 19^{\prime} \mathrm{N}, 45^{\circ} 41^{\prime} 48^{\prime \prime} \mathrm{E}, 2160 \mathrm{~m}$, stream valley, rose bushes, litter and roots sifted, 10.VII.2017; 84: $30 \mathrm{~km}$ NW Sisian, Mt. Karkar, 39 46'38"N, 4556'04"E, 2970 m, pasture, mouse nest under stone sifted, 11.VII.2017; 85: 30 km

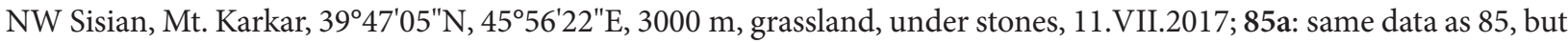
mouse nests sifted; $85 \mathrm{~b}$ : same data as 85 , but horse dung sifted; $85 \mathrm{c}$ : same data as 85 , but wetland, moist debris near water sifted; 86: $30 \mathrm{~km}$ NW Sisian, Mt. Karkar, 3946'51"N, 4556'30"E, 2990 m, straw manure sifted, 11.VII.2017;

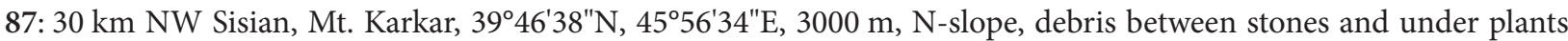
sifted, 11.VII.2017; 87a: same data as 87, but grassy N-slope with rocks, under stones; 88: NW Goris, W Verishen, $39^{\circ} 31^{\prime} 43^{\prime \prime} \mathrm{N}, 46^{\circ} 18^{\prime} 33^{\prime \prime E}, 1670 \mathrm{~m}$, margin of oak forest, litter, moss, and roots beneath Quercus and Rosa sifted, 12.VII.2017; 89: SW Goris, ESE Tatev, 39²1'47"N, 46²16'52"E, $1950 \mathrm{~m}$, margin of mixed deciduous forest (Quercus, Carpinus, Acer, etc.), litter and roots sifted, 13.VII.2017; 89a: same data as 89, but nests of Formica exsecta sifted; 89b: same data as 89, but 14.VII.2017; 90: SW Goris, ESE Tatev, 39²1'32"N, 46 $16^{\prime} 05^{\prime \prime} \mathrm{E} 1730 \mathrm{~m}$, margin of mixed deciduous forest (Quercus, Carpinus, Acer, etc.), litter and debris in or near small ditch near road sifted, 13.VII.2017; 90a: same data as 90, but mixed deciduous forest (Quercus, Carpinus, Acer, etc.), stream valley, moist litter near small stream sifted; 91: SW Goris, ESE Tatev, 39²1'50"N, 46'16'32"E $1820 \mathrm{~m}$, margin of mixed deciduous forest (Quercus, Carpinus, Acer, etc.), litter and debris between rocks sifted, 14.VII.2017.

92-131: field trip Armenia and Nagorno-Karabakh, 2018, leg. Assing \& Schülke [92-97, 118-131: Armenia; 98-117:

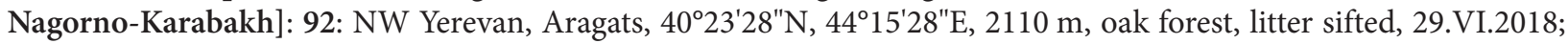
93: NW Yerevan, Aragats, $40^{\circ} 24^{\prime} 22^{\prime \prime} \mathrm{N}, 44^{\circ} 13^{\prime} 40^{\prime E} \mathrm{E}, 2200 \mathrm{~m}$, moist slope, sifted, 29.VI.2018; 93a: same data as 93, but

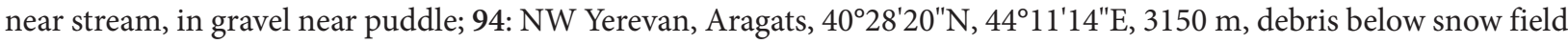
sifted, 29.VI.2018; 94a: same data as 94, but meadow near lake and slope below snowfield, under stones; 95: N Yere-

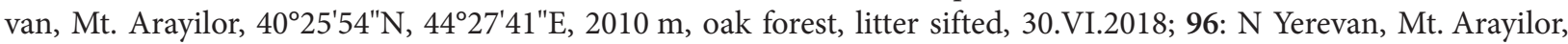

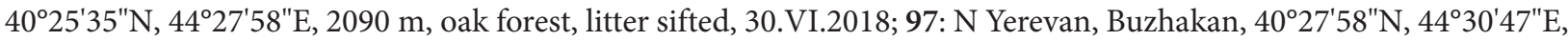
1930 m, oak forest, litter sifted, 30.VI.2018; 98: ca $12 \mathrm{~km} \mathrm{NW}$ Stepanakert, 3954'22"N, 46³9'12"E, $870 \mathrm{~m}$, mixed forest with Fagus, Acer, Tilia, Corylus, etc., litter sifted, 2.VII.2018; 99: ca. 12 km NW Stepanakert, 3954'58"N, 46² $40^{\prime} 14^{\prime \prime} \mathrm{E}$, $810 \mathrm{~m}$, stream bank, floated from gravel, 2.VII.2018; 100: ca. 40 NW Stepanakert, W Vank, $40^{\circ} 01^{\prime} 04^{\prime \prime} \mathrm{N}, 46^{\circ} 26^{\prime} 32^{\prime \prime} \mathrm{E}$, $1420 \mathrm{~m}$, S-slope with mixed deciduous forest, moist litter near small stream sifted, 3.VII.2018; 101: ca. $40 \mathrm{~km}$ NW Stepanakert, W Vank, $40^{\circ} 01^{\prime} 34^{\prime \prime} \mathrm{N}, 46^{\circ} 28^{\prime} 33^{\prime \prime} \mathrm{E}, 1210 \mathrm{~m}$, deciduous forest on river bank, litter sifted, 3.VII.2018; 101a: same data as 101, but deciduous forest, debris and litter near small waterfall sifted; 102: ca. $40 \mathrm{~km}$ NW Stepanakert, W Vank, $40^{\circ} 01^{\prime} 44^{\prime \prime} \mathrm{N}, 46^{\circ} 30^{\prime} 15^{\prime \prime E}, 1080 \mathrm{~m}$, mixed deciduous forest, dry leaf litter sifted, 3.VII.2018; 103: ca. $75 \mathrm{~km}$ WNW Stepanakert, S Karvachar, $40^{\circ} 02^{\prime} 02^{\prime \prime N}, 46^{\circ} 00^{\prime} 10^{\prime \prime} \mathrm{E}, 1630 \mathrm{~m}$, degraded mixed deciduous forest with small stream, litter sifted, 4.VII.2018; 104: ca. 75 km WNW Stepanakert, S Karvachar, 4001'24"N, 4559'28"E, 1680 m, river bank, Salix litter sifted, 4.VII.2018; 105: ca. 50 km WNW Stepanakert, S Dadivank, 3959'13"N, 46 $14^{\prime} 45^{\prime \prime} \mathrm{E}, 1610 \mathrm{~m}$, mixed deciduous forest margin, litter and roots sifted, 5.VII.2018; 106: ca. $50 \mathrm{~km}$ WNW Stepanakert, S Dadivank, $40^{\circ} 00^{\prime} 40^{\prime \prime} \mathrm{N}, 46^{\circ} 14^{\prime} 00^{\prime \prime} \mathrm{E}$, 
$1560 \mathrm{~m}$, mixed deciduous forest with small stream, moist litter and debris near stream sifted, 5.VII.2018; 107: pass $20 \mathrm{~km}$ SW Stepanakert, 39 $40^{\prime} 40^{\prime \prime} \mathrm{N}, 46^{\circ} 39^{\prime} 56^{\prime \prime} \mathrm{E}, 1940 \mathrm{~m}$, stream bank, litter and roots sifted, 6.VII.2018; 108: pass $20 \mathrm{~km}$ SW Stepanakert, 39 $41^{\prime} 41^{\prime \prime} \mathrm{N}, 46^{\circ} 39^{\prime} 02^{\prime \prime} \mathrm{E}, 1760 \mathrm{~m}$, secondary mixed forest with pine and oak, 6.VII.2018; 109: pass $20 \mathrm{~km}$ SW Stepanakert, 39 $41^{\prime} 35^{\prime \prime} \mathrm{N}, 46^{\circ} 39^{\prime} 00^{\prime \prime E}, 1760 \mathrm{~m}$, stream bank with Salix and herbs, litter and roots

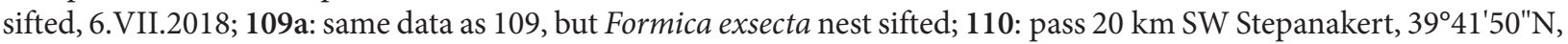
$46^{\circ} 39^{\prime} 00^{\prime \prime E}, 1730 \mathrm{~m}$, stream valley with mixed deciduous forest, predominantly oak, litter sifted, 6.VII.2018; 111: Step-

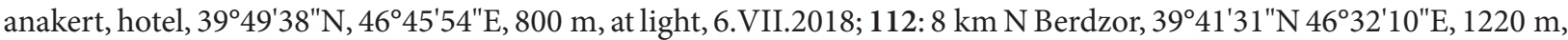
$\mathrm{N}$-slope with mixed deciduous forest and small stream, litter and debris near stream sifted, 7.VII.2018; 113: pass $20 \mathrm{~km}$ SW Stepanakert, 39 $42^{\prime} 13^{\prime \prime} \mathrm{N} 46^{\circ} 39^{\prime} 02^{\prime \prime E}, 1700 \mathrm{~m}$, stream valley with deciduous trees, litter near stream and near rocks sifted, 7.VII.2018; 114: $13 \mathrm{~km}$ W Hadrut, 39³1'05"N, 4654'19"E, $1760 \mathrm{~m}$, mixed deciduous forest with Quercus, Acer, Carpinus, Corylus, litter in and near moist stream bed sifted, 8.VII.2018; 115: 8 km W Hadrut, 39³1'52"N 4657'50"E, $1480 \mathrm{~m}$, stream valley with mixed deciduous forest, moist litter near stream sifted, 8.VII.2018; 116: mountain ca. $10 \mathrm{~km}$ SE Stepanakert, 3947'03"N, 4651'02"E, $1520 \mathrm{~m}$, mixed deciduous forest, litter sifted, 9.VII.2018; 117: ca. $30 \mathrm{~km}$

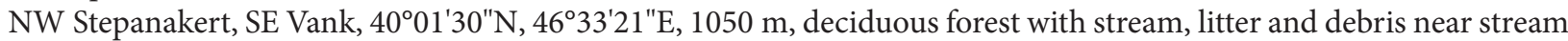
sifted, 9.VII.2018; 118: WSW Kapan, W Kajaran, 3909'22"N, 4606'13"E, 2050 m, steep N-slope with mixed deciduous forest, leaf litter and grass roots sifted, 10.VII.2018; 118a: same data as 118, but 13.VII.2018; 119: ca. $30 \mathrm{~km}$ W Kapan, $39^{\circ} 15^{\prime} 13^{\prime \prime} \mathrm{N}, 46^{\circ} 04^{\prime} 16^{\prime \prime} \mathrm{E}, 2040 \mathrm{~m}$, N-slope, margin of mixed deciduous forest, litter and grass roots sifted, 11.VII.2018; 120: ca. $30 \mathrm{~km}$ W Kapan, $39^{\circ} 14^{\prime} 40^{\prime \prime} \mathrm{N}, 46^{\circ} 06^{\prime} 24^{\prime \prime} \mathrm{E}, 1780 \mathrm{~m}$, river bank, floated from gravel, 11.VII.2018; 121: ca. $30 \mathrm{~km}$ W Kapan, 39 $14^{\prime} 36^{\prime \prime} \mathrm{N}, 46^{\circ} 08^{\prime} 28^{\prime \prime} \mathrm{E}, 1680 \mathrm{~m}$, N-slope with trickling water, moist litter and debris sifted, 11.VII.2018; 121a: same data as 121, but river bank, litter and debris sifted; 122: SW Kapan, Mt. Khustup, 3907' $52^{\prime \prime} \mathrm{N}$, $46^{\circ} 19^{\prime} 43^{\prime \prime E}, 3060 \mathrm{~m}$, N-slope, soil and roots of Rumex sp. near large rocks sifted, 12.VII.2018; 123: SW Kapan, Mt. Khustup, 39 $07^{\prime} 50^{\prime \prime} \mathrm{N}, 46^{\circ} 19^{\prime} 55^{\prime E} \mathrm{E}, 2980 \mathrm{~m}$, litter near spring sifted, 12.VII.2018; 124: SSW Kapan, forest above Shishkert, 390' $48^{\prime \prime} \mathrm{N}, 46^{\circ} 21^{\prime} 36^{\prime \prime} \mathrm{E}, 2040 \mathrm{~m}$, roots, litter, and stony soil sifted, 12.VII.2018; 125: WSW Kapan, S Lernadzor, $39^{\circ} 08^{\prime} 02^{\prime \prime} \mathrm{N}, 46^{\circ} 13^{\prime} 07^{\prime \prime E}, 2000 \mathrm{~m}$, dark stream valley, litter and roots near stream sifted, 13.VII.2018; $126: 25 \mathrm{~km}$ SW Kapan, Vank, 39 $04^{\prime} 01^{\prime \prime} \mathrm{N}, 46^{\circ} 16^{\prime} 10^{\prime \prime} \mathrm{E}, 2150 \mathrm{~m}$, near stream, litter of Salix, roots, and debris sifted, 14.VII.2018; 127: $25 \mathrm{~km}$ S Goris, ESE Tatev, 39²0'18"N, 46²2'06"E, $1590 \mathrm{~m}$, mixed deciduous forest with small stream, moist litter

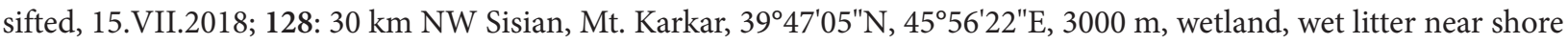
of lake sifted, 15.VII.2018; 129: 20 km SW Sisian, Darbas, 39² $25^{\prime} 55^{\prime \prime} \mathrm{N}, 46^{\circ} 06^{\prime} 57^{\prime \prime E}, 1680 \mathrm{~m}$, margin of secondary deciduous forest, litter and soil sifted, 16.VII.2018; 130: reservoir $15 \mathrm{~km} \mathrm{SW}$ Sisian, 39²8'18"N, 46 $07^{\prime} 57^{\prime \prime} \mathrm{E}, 1340 \mathrm{~m}$, shore of reservoir, Salix litter, moss, and roots sifted, 16.VII.2018; 131: $35 \mathrm{~km}$ SSE Martuni, Arates, 39 $54^{\prime} 28^{\prime \prime} \mathrm{N}, 4^{\circ} 26^{\prime} 07^{\prime \prime} \mathrm{E}$, $2040 \mathrm{~m}$, margin of small forest patch with small stream, litter and roots sifted, 17.VII.2018.

132-144: field trip Armenia, 2016, leg. A. \& J. Müller: 132: Ditavan env., 4058'49"N, 45¹1'57"E, 610 m, 21.V.2016; 133: Ditavan env., $40^{\circ} 56^{\prime} 45^{\prime \prime} \mathrm{N}, 45^{\circ} 12^{\prime} 20^{\prime E} \mathrm{E}, 1240 \mathrm{~m}, 22 . \mathrm{V} .2016 ; 134$ : Ditavan env., $40^{\circ} 58^{\prime} 07^{\prime \prime} \mathrm{N}, 45^{\circ} 12^{\prime} 08^{\prime \prime} \mathrm{E}, 740 \mathrm{~m}$, 22.V.2016; 134a: same data as 134, but 23.V.2016; 134b: Ditavan env., $40.94750^{\circ} \mathrm{N}, 45.20793^{\circ} \mathrm{E}, 1290 \mathrm{~m}, 28 . I X .2016$;

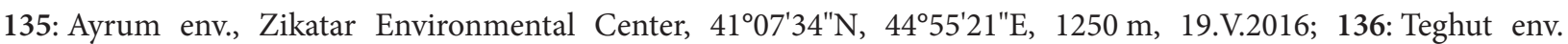
(Akhtala), $41.091^{\circ} \mathrm{N}, 44.813^{\circ} \mathrm{E}, 1020 \mathrm{~m}, 20 . \mathrm{V} .2016 ; 137$ : Teghut env. (Dilijan), $41^{\circ} 05^{\prime} 26^{\prime \prime} \mathrm{N}, 44^{\circ} 48^{\prime} 51^{\prime \prime} \mathrm{E}, 1340 \mathrm{~m}$,

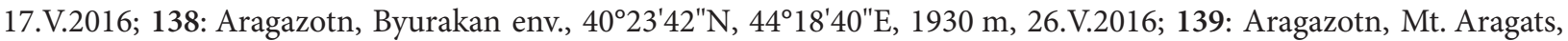

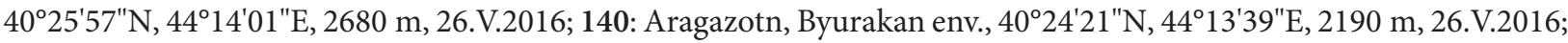

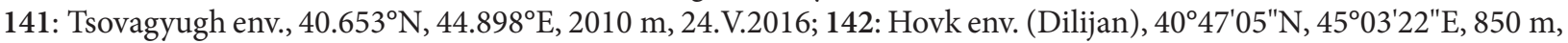

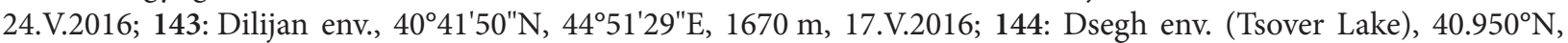
$44.685^{\circ} \mathrm{E}, 1390 \mathrm{~m}, 23 . \mathrm{V} .2016$.

145-160: Armenia, 2013-2017, leg. Kalashian, Grejyan \& Karagyan: 145: Vayotsdzor prov., Amulsar mine E of Jermuk and Gndevaz, 39.74646 ${ }^{\circ} \mathrm{N}$, 45.66483 ${ }^{\circ} \mathrm{E}, 2040 \mathrm{~m}$, 28.VI.-18.VII.2017; 145a: same data as 145, but 18.VII.-7.VIII.2017; 145b: same data as 145, but 7.VIII.-23.IX.2017; 146: Vayotsdzor prov., Amulsar mine E of Jermuk and Gndevaz, $39.7335^{\circ} \mathrm{N}, 45.6664^{\circ} \mathrm{E}, 1985 \mathrm{~m}$, 7.VIII.-23.IX.2017; 146a: same data as 146, but 18.VII.- 7.VIII.2017; 147: Vayotsdzor prov., Amulsar mine E of Jermuk and Gndevaz, $39.72721^{\circ} \mathrm{N}, 45.71643^{\circ} \mathrm{E}, 2975 \mathrm{~m}, 18$. VII.- 7.VIII.2017; 148: Lori prov., Teghut mine, near artificial pond, $41.0907^{\circ} \mathrm{N}, 44.8117^{\circ} \mathrm{E}, 990 \mathrm{~m}$, soil traps, 28.IV.-25.V.2017; 148a: same data as 148, but 25.V.-23.VII.2017; 148b: same data as 148, but 23.VII.-13.VIII.2017; 148c: same data as 148, but 13.VIII.-13.X.2017; 148d: same data as 148, but 19.VI.-23.VII.2015; 148e: same data as 148, but 23.VII.-23.VIII.2015; 148f: same data as 148, but 1.-24.V.2015; 148g: same data as 148, but 23.VIII.-23.IX.2015; 149: Lori prov., Teghut mine, near refuse heap, $41.0705^{\circ} \mathrm{N}, 44.8378^{\circ} \mathrm{E}, 1210 \mathrm{~m}$, soil traps, 28.IV.-25.V.2017; 149a: same data as 149, but 23.VII.-13.VIII.2017; 149b: same data as 149, but 13.VIII.-13.X.2017; 150: Lori prov., Teghut mine, Dukanadzor gorge, $41.0910^{\circ} \mathrm{N}, 44.8514^{\circ} \mathrm{E}$, 890 m, soil traps, 28.IV.-25.V.2017; 150a: same data as 150, but 25.V.-23.VII.2017; 150b: same data as 150, but 19.VI.23.VII.2015; 150c: same data as 150, but 1.-24.V.2014; 150d: same data as 150, but 23.VIII.-23.IX.2015; 151: Lori prov., Teghut mine, Dukanadzor gorge, Kharatanots gorge, $41.0964^{\circ} \mathrm{N}, 44.8219^{\circ} \mathrm{E}, 910 \mathrm{~m}$, soil traps, 28.IV.-25.V.2017; 151a: same data as 151, but 25.V.-23.VII.2017; 151b: same data as 151, but 23.VII.-13.VIII.2017; 151c: same data as 151, but 19.VI.-23.VII.2015; 151d: same data as 151, but $920 \mathrm{~m}, 1 .-4 . V .2014$; 152: Lori prov., Teghut mine, $920 \mathrm{~m}$, 20.VI.-1.VIII.2013; 153: Lori prov., Teghut mine, 1100 m, 22.VIII.-1.X.2013; 154: Lori prov., Teghut mine, tailing 
dump, $1100 \mathrm{~m}$, soil traps, 1.-24.V.2014; 155: Syunik prov., $\sim 5 \mathrm{~km} \mathrm{~N}$ Shvanidzor, $38.9851^{\circ} \mathrm{N}, 46.3746^{\circ} \mathrm{E}, 1335 \mathrm{~m}$, soil traps, 9.VI.-8.VIII.2017; 156: Syunik prov., between Tandzaver and Aghvani, $39.3499^{\circ} \mathrm{N}, 46.3164^{\circ} \mathrm{E}, 1670 \mathrm{~m}, 5-30$. VII.2015; 157: Syunik prov., N Verin Khotanan, 39.3284N, 46.3751 ${ }^{\circ} \mathrm{E}, 1660$ m, 5-30.VII.2015; 158: Syunik Province, S Dastakert, 1930 m, 29.VII.2011; 159: Vayotsdzor Province, E Gndevaz, 1980 m, soil traps, 5-22.VI.2014; 160: Vayotsdzor Province, E Gndevaz, 2190 m, soil traps, 5.-22.VI.2014.

161-174: 2015, Armenia, leg. Kocian: 161: Dashtakar env., Vedi river, $39.920756^{\circ} \mathrm{N}, 44.782199^{\circ} \mathrm{E}, 1000 \mathrm{~m}$, river bank, 18.V.2015; 162: Lanjanist env., $39.859084^{\circ} \mathrm{N}, 44.937384^{\circ} \mathrm{E}, 1560 \mathrm{~m}$, leaf litter and dead wood sifted, 18.V.2015; 163: Jermuk env., $39.814797^{\circ} \mathrm{N}, 45.644753^{\circ} \mathrm{E}, 2060 \mathrm{~m}$, W slope, deciduous forest, sifted, 19.V.2015; 164: Yeghegis env., $39.871366^{\circ} \mathrm{N}, 45.411616^{\circ} \mathrm{E}, 1700 \mathrm{~m}, \mathrm{~N}$ slope, deciduous forest, sifted, $120 . \mathrm{V} .2015 ; 165$ : above Jermuk, $39.839053^{\circ} \mathrm{N}$, $45.693496^{\circ} \mathrm{E}, 2400 \mathrm{~m}$, plant debris near snow fields sifted, 21.V.2015; 166: Jermuk env., $39.806823^{\circ} \mathrm{N}, 45.650697^{\circ} \mathrm{E}$, $2000 \mathrm{~m}, 19 . \mathrm{V} .2015 ; 167$ : Artavan valley, $39.676737^{\circ} \mathrm{N}, 45.569628^{\circ} \mathrm{E}, 1530 \mathrm{~m}$, river bank, leaf litter sifted, 22.V.2015; 168: Vorotan pass, $39.681390^{\circ} \mathrm{N}, 45.720964^{\circ} \mathrm{E}, 2250 \mathrm{~m}$, grass and debris sifted, 23.V.2015; 169: Tatev env., Vorotan valley, $39.394394^{\circ} \mathrm{N}, 46.247672^{\circ} \mathrm{E}, 1300 \mathrm{~m}$, macchia, sifted, 24.V.2015; 170: Goris, $39.516611^{\circ} \mathrm{N}, 46.322847^{\circ} \mathrm{E}, 1700 \mathrm{~m}$, E slope, deciduous forest, leaf litter sifted, 25.V.2015; 171: Goris env. Khndzoresk, old cemetery, $39.502770^{\circ} \mathrm{N}$, $46.428540^{\circ} \mathrm{E}, 1400 \mathrm{~m}$, macchia, sifted, 26.V.2015; 172: Goris env., above Verishen, $39.558515^{\circ} \mathrm{N}, 46.316772^{\circ} \mathrm{E}, 1880 \mathrm{~m}$, sifted, 26.V.2015; 173: Vorotan pass, $39.686492^{\circ} \mathrm{N}, 45.693326^{\circ} \mathrm{E}, 2160 \mathrm{~m}$, grass and plant debris sifted, 27.V.2015; 174: $3 \mathrm{~km} \mathrm{~S}$ Nshkhark, $39.971887^{\circ} \mathrm{N}, 45.241237^{\circ} \mathrm{E}, 2280 \mathrm{~m}$, grass and plant sifted, 27.V.2015.

175-213: 2001, Armenia, leg. Shaverdo \& Schillhammer: 175: N Yerevan, SW Carencavan, SE Lusakert, Hrazdan river, 1280 m, 30.IV.2001; 176: E Yerevan, approximately halfway between Yerevan and Garni, below Atsavan, 1300 m, lake with reed, 1.V.2001; 177: SE Yerevan, Azat reservoir near Bartsrashen, close to inflow of river, $1040 \mathrm{~m}$,

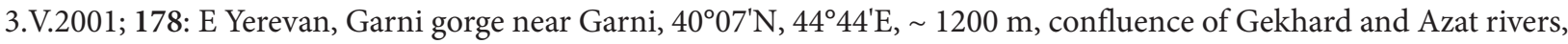

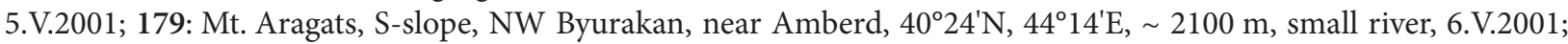

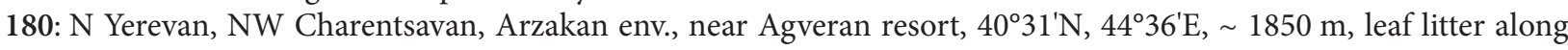

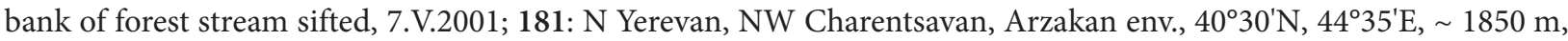
very wet leaf litter along bank of small tributary to "Arzakan" river sifted, 7.V.2001; 182: N Yerevan, 24 km NW Hrazdan,

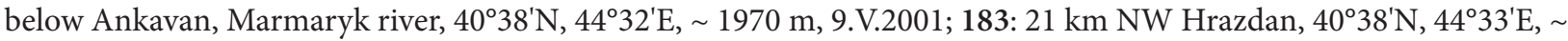

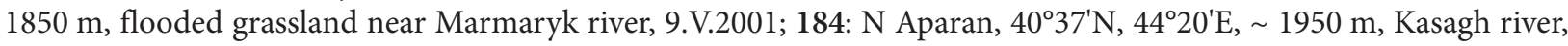
10.V.2001; 185: Mt. Aragats, S-slope, Amberd river above Byurakan, $40^{\circ} 22^{\prime} \mathrm{N}, 44^{\circ} 15^{\prime} \mathrm{E}, \sim 1580 \mathrm{~m}$, near river bank, 10.V.2001; 186: SE Yerevan, E Yeghegnadzor, $7 \mathrm{~km}$ E Bazarchay, Vorotan river above Sisian reservoir, $39^{\circ} 41^{\prime} \mathrm{N}, 45^{\circ} 47^{\prime} \mathrm{E}$, 2050 m, 12.V.2001; 187: SE Yerevan, E Yeghegnadzor, 2 km W Karmrashen, 3950'N, 45³1'E, 2050 m, stream flowing through alpine plain, 13.V.2001; 188: SE Yerevan, NE Yeghegnadzor, E Gyullidus, Gyadikvank env., Yeghegis river, $39^{\circ} 53^{\prime} \mathrm{N}, 45^{\circ} 29^{\prime} \mathrm{E}, \sim 1850 \mathrm{~m}$, river bank with Salix, 13.V.2001; 189: SE Yerevan, NE Areni, W Lusashogh, 3952' N, $44^{\circ} 57^{\prime} \mathrm{E}$, $1700 \mathrm{~m}$, pond, 14.V.2001; 190: W Yerevan, SW Echmiadzin, Yerasghaun env., $40^{\circ} 05^{\prime} \mathrm{N}, 44^{\circ} 12^{\prime} \mathrm{E}, \sim 850 \mathrm{~m}$, 15.V.2001; 191: SE Yerevan, Khosrov Nature Reserve, 3959'N, 4453'E, 1300-1500 m, 16-17.V.2001; 191a: same as 191,

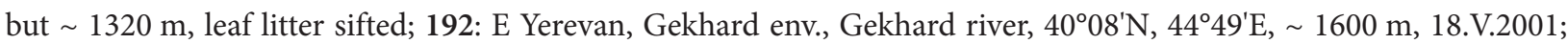
193: E Yerevan, Gekhard env., between Gekhard and Goght, 1600-1800 m, 18.V.2001; 194: 32 km SE Vanadzor, above Fioletovo, $40^{\circ} 43^{\prime} \mathrm{N}, 44^{\circ} 44^{\prime} \mathrm{E}, \sim 1730 \mathrm{~m}, 20 . \mathrm{V} .2001$; 195: NE Dilijan, near Haghartsin monastery, $40^{\circ} 48^{\prime} \mathrm{N}, 44^{\circ} 53^{\prime} \mathrm{E}, \sim$ $1450 \mathrm{~m}$, along riverbanks and under stones on slopes, 21.V.2001; 195a: same as 195, but sifted from dripping wet leaf

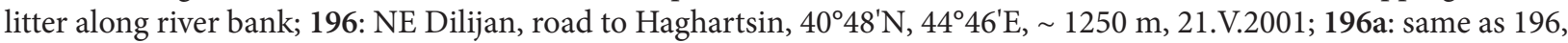
but leaf litter sifted; 197: same as 196, but $40^{\circ} 48^{\prime} \mathrm{N}, 44^{\circ} 54^{\prime} \mathrm{E}, \sim 1300 \mathrm{~m}$, deep car tracks; 198: E Dilijan, Parzlich Lake, $40^{\circ} 45^{\prime} \mathrm{N}, 44^{\circ} 58^{\prime} \mathrm{E}, \sim 1330 \mathrm{~m}$, puddle in forest, 22.V.2001; 199: E Dilijan, below Gosh, $40^{\circ} 44^{\prime} \mathrm{N}, 45^{\circ} 01^{\prime} \mathrm{E}, \sim 1000 \mathrm{~m}$, bank of small stream, 22.V.2001; 199a: same as 199, but sifted from very wet leaf litter at base of slope along bank of stream; 199b: same as 199, but under stones near stream; 200: NE-shore of Sevan Lake, 40³6'N, 4504'E, $1940 \mathrm{~m}$, from vegetation; 201: ca $10 \mathrm{~km} \mathrm{~N}$ Sevan, Tzovagyugh env., 40³6' $\mathrm{N}, 44^{\circ} 57^{\prime} \mathrm{E}, \sim 1940 \mathrm{~m}$, large swampy area and flooded meadow along channels, 22.V.2001; 202: same as 182, but 27.V.2001; 203: same as 183, but 27.V.2001; 203a: same as 203, but under stones; 204: same as 179, but 28.V.2001; 204a: same as 204, but terrestrial; 205: 25 km N Kapan, Nor Arajadzor env., SW-shore of water reservoir "Davidbeksoe", 1400 m, lakeshore, 30.V.2001; 206: 25 km N Kapan, Nor Arajadzor env., Khashuni river bank, 30.V.2001; 207: between Kapan and Kajaran, Darmanadzor (Aramazd) gorge, 1700 m, under stones, 30.V.2001; 208: 3 km W Kadzharan, "Old Kajaran” env., 2000 m, puddles on the banks of Kajaran river, 31.V.2001; 209: 16 km SW Kajaran, 2130 m, stream, 31.V.2001; 210: same as 209, but puddle near larger stream; 211: SSE Sisian, Lernashen env., $1870 \mathrm{~m}$, under stones, 1.VI.2001; 212: SE Yerevan, ca $10 \mathrm{~km}$ E Artashat, foot of Mt. Otsasar, 1050 m, semi-desert, 2.VI.2001; 213: E Yerevan, Gekhard river near Garni, 1200 m, 29.IV.2001.

Additional records, Armenia: a: $12 \mathrm{~km}$ SE Armavir, Arazap env., Araratskaya Koshenil Reserve, salt marsh, 400 $04^{\prime} \mathrm{N}$, $44^{\circ} 08^{\prime} \mathrm{E}, 850 \mathrm{~m}$, 13.VI.2017, leg. Šumpich (NMP, cAss); b: $3.2 \mathrm{~km} \mathrm{NW} \mathrm{Meghri,} \mathrm{Artsvakar,} \mathrm{Arevik} \mathrm{National} \mathrm{Park,}$ $38^{\circ} 55^{\prime} \mathrm{N}, 46^{\circ} 16^{\prime} \mathrm{E}, 750 \mathrm{~m}$, rocky steppe, gorge, 6-7.VI.2017, leg. Šumpich (NMP); c: Vedi env., Goravan, Goravan Sands Reserve, 960 m, 38 $55^{\prime} \mathrm{N}, 44^{\circ} 44^{\prime} \mathrm{E}$, sandy steppe, 31.VI.2017, leg. Šumpich (NMP); d: Shvanidzor env., Arevik National

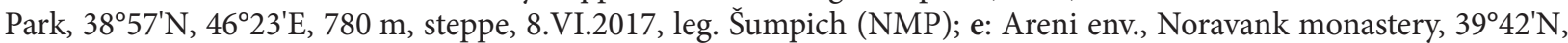
$45^{\circ} 13^{\prime} \mathrm{E}, 1330$ m, 10.VI.2017, leg. Šumpich (NMP); f: 2.3 km NW Aygedzor, Lichtkvaz, Arevik National Park, 3859'N, 
46ํำ $11^{\prime} \mathrm{E}, 1335$ m, mountain steppe, 4.VI.2017, leg. Šumpich (NMP); g: 3 km NW Meghri, Lehvaz env., Arevik National Park, 3855'N, 46²13'E, 840 m, rocky steppe, 5.VI.2017, leg. Šumpich (NMP); h: 5.5 km N Shvanidzor, 4 km S Gomarants pass, Arevik National Park, 3859'N, 46²2'E, 1330 m, 9.VI.2017, leg. Šumpich (NMP); i: 13 km SE Yerevan,

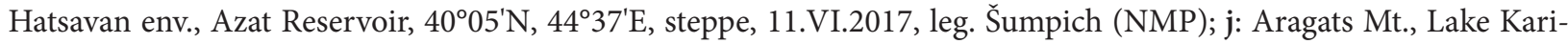
litsh, 3300 m, 21.IX.1982, leg. Merkl et Ronkay (MNB); k: Tsakhkadzor, 2800 m, under stones, 1.X.1982, leg. Merkl \& Ronkay (MNB); l: Aragats Mt., 3200 m, 27.VIII.1976, leg. Vásárhelyi (HNHM); m: Taakhkadzor, 1800-2300 m, 1.X.1982, leg. Merkl \& Ronkay (HNHM); n: Yerevan, 11.V.1951, leg. Khnzorian (cKhn); o: Kotayk province, Tsakhkadzor, 17.IV.1978, leg. Khnzorian (cKhn); p: Lori province, "Kirowakan, Chagali” [= Vanadzor], 2.VI.1948, leg. Khnzorian (cKhn); q: Lori province, Stepanavan, "Yagan”, 17.VI.1955, leg. Khnzorian (cKhn); r: Kotayk province, Tsakhkadzor, 10.V.1949, leg. Khnzorian (cKhn); s: Lori province, Vanadzor, 2.VI.1949, leg. Khnzorian (cKhn); t: Goris, "Shurnukhi”, 9.VIII.1950, leg. Khnzorian (cKhn); u: Tavush province, Ijevan, 24.V.1951, leg. Khnzorian (cKhn); v: Yerevan, Jrvezh, 9.VI.1952, leg. Khnzorian (cKhn); w: Tavush province, Alaverdi, Akhtala, 6.VI.1940, leg. Khnzorian (cKhn); x: "Basargenar, Mazr. Kochewki”, 22.VII.1958, leg. Khnzorian (cKhn); y: Syunik province, Kapan, 18.VII.1952, leg. Khnzorian (cKhn); z: Yerevan, zoo, 16.XI.1952, leg. Khnzorian (cKhn); aa: Yerevan, Jrvezh, 9.VI.1952, leg. Khnzorian (cKhn); ab: Tavush province, Dilijan, Haghartsin, 10.V.1975, leg. Khnzorian (cKhn); ab: Tavush province, Dilijan, Haghartsin, 10.V.1975, leg. Khnzorian (cKhn); ac: Lori province, Vanadzor, "Magaly", 1.VI.1949, leg. Khnzorian (cKhn); ad: Kotayk province, Yerevan, Geghard, 3.V.1950, leg. Khnzorian (cKhn); ae: Yerevan, “Bushakan”, 25.VI.1959, leg. Khnzorian (cKhn); af: Aragats, "Karilik", 29.VIII.1948, leg. Khnzorian (cKhn); ag: Alaverdi, Sanahin, 5.VI.1949, leg. Khnzorian (cKhn); ah: Lori province, Stepanavan, Gyulagarak, 18.VII.1954, leg. Khnzorian (cKhn); ai: Sevan, "Khitapalar”, 1.VIII.1948, leg. Khnzorian (cKhn); aj: Gegharkunik province, Sevan lake, Chambarak, 2700 m, 11.VI.1949, leg. Khnzorian (cKhn); ak: Argatsotn province, Aragats, K'ari Lich, 29.VIII.1948, leg. Khnzorian (cKhn); al: Vayots Dzor province, Yeghegnadzor, Arpa river, 20.VII.1949, leg. Khnzorian (cKhn); am: Syunik province, Kapan, Vachagan [39 $11^{\prime} \mathrm{N} 46^{\circ} 23^{\prime} \mathrm{E}$ ], 16.VII.1952, leg. Khnzorian (cKhn); an: Vayots Dzor province, Yeghegnadzor, Shatin [3950'N, $\left.45^{\circ} 18^{\prime} \mathrm{E}\right]$, 16.VII.1950, leg. Khnzorian (cKhn); ao: Lori province, Vanadzor, 2.VI.1949, leg. Khnzorian (cKhn); ap: Lori province, Vanadzor, "Vartanidzor", 20.V.1950, leg. Khnzorian (cKhn); aq: Lori province, Vanadzor, Pambak range, Mt. Maimekh, 21.V.1950, leg. Khnzorian (cKhn); ar: Yerevan, “Digrliuz”, 14.IV.1950, leg. Khnzorian (cKhn).

\section{References:}

A97 = Assing (1997); A99 = Assing (1999); A03 = Assing (2003); A04a = Assing (2004a); A05a = Assing (2005a); A05b = Assing (2005b); A06 = Assing (2006); A07b = Assing (2007b); A08a = Assing (2008a); A08b = Assing (2008b); A08c = Assing (2008c); A09a = Assing (2009a); A10a = Assing (2010a); A10b = Assing (2010b); A13a = Assing (2013a); A13b = Assing (2013b); A14 = Assing (2014); A15 = Assing (2015); A16a = Assing (2016a); A16b $=$ Assing (2016b); A16c = Assing (2016c); A17a = Assing (2017a); A17b = Assing (2017b); A17c = Assing (2017c); A17d = Assing (2017d); A17e = Assing (2017e); A17f = Assing (2017f); A17g = Assing (2017g); A18a = Assing (2018a); A18b = Assing (2018b); A18c = Assing (2018c); A18d = Assing (2018d); A18e = Assing (2018e); A18f = Assing (2018f); A18g = Assing (2018g); A18h = Assing (2018h); A19a = Assing (2019a); A19b = Assing (2019b); AKÖ11 = Anlaş et al. (2011); Am99 = Amiryan (1999a); Am99b = Amiryan (1999b); Am00 = Amiryan (2000); An17 = Anlaş (2017); An18 = Anlaş (2018); ASpp = Assıng \& SchülKe (present paper); ASo98 = Assing \& SoloDOVNIKov (1998); ATHW00 = AMIRYAN et al. (2000); AV17 = Assing \& Vogel (2017); AW08 = Assing \& WUNDERLE (2008); Be74 = Benick (1974); BK07 = Besuchet \& Kurbatov (2007); Bo84 = Bordoni (1984); Bo11a = Bordoni (2011a); Bo11b = Bordoni (2011b); Bo17 = Bordoni (2017); Br01 = BERnHAUER (1901); Br08 = BernHAUER (1908); Br44 = Bernhauer (1944); BrS10 = BernhAuER \& SCHUBERT (1910); BrS12 = BERNHAuER \& SCHUBERT (1912), BrS14 = Bernhauer \& Schubert (1914); BrSz26 = Bernhauer \& Scheerpeltz (1926); BS99 = Besuchet \& Sabella (1999); C56 = Coiffait (1956); C60 = Coiffait (1960); C65 = Coiffait (1965); C66a = Coiffait (1966a); C66b = Coiffait (1966b); C67a = Coiffait (1967a); C67b = Coiffait (1967b); C68 = Coiffait (1968); C69 = CoIfFAIT (1969); C70a = CoIFFAIT (1970a); C70b = CoIFFAIT (1970b); C70c = CoIFFAIT (1970c); C70d = CoIFFAIT (1970d); C72a = Coiffait (1972a); C72b = Coiffait (1972b); C74 = CoIfFAit (1974); C77 = CoIfFait (1977); Ca39 = CAMERON (1939); Da04 = DAvies (2004); Dv84 = Dvoř́́K (1984); E30 = EICHLER (1930); Ep78 = EpPELSHEIM (1878); Ep90b = Eppelsheim (1890b); ES16 = Enushchenko \& Semenov (2016); Fa71 = Fauvel (1871); Fa72 = Fauvel (1872); Fa74 = FAUVEL (1874); Fr97 = FrISCH (1997); Fr07a = FrisCH (2007a); Fr07b = FrisCH (2007b); Fr09 = FrISCH (2009); Fr10 = Frisch (2010); Fr12 = Frisch (2012); G00 = Gildenkov (2000); G01 = GildenKov (2001); G09 = GiLdENKOv (2009); G15 = GiLdENKov (2015); H01 = Herman (2001); H86 = HermaN (1986); Hh49 = HochHUTH (1849); Hh51 = Hochнuтн (1851); Hh62 = HochHUTH (1872); HMM79 = HAMMOND et al. (1979); Hn65 = HorION (1965); Hr90 = HROMÁdKA (1990); IK56 = IABLOKOFF-KHNZORIAN (1956); IK57a = IABLOKOFF-KHNZORIAN (1957a); IK57b = IABLOKOFF-KHNZORIAN (1957b); IK57c = IABLOKOFF-KHNZORIAN (1957c); IK59 = IAbLOKOFF-KHNZORIAN (1959); IK60 = IABLOKOFF-KHNZORIAN (1960);IK61 = IABLOKOFF-KHNZORIAN (1961); IK62 = IABLOKOFF-KHNZORIAN(1962); IK64a = IABLOKOFF-KHNZORIAN (1964a); IK64b = IABLOKOFF-KHNZORIAN (1964b); IK66 = IABLOKOFF-KHNZORIAN (1966); IK85 = IABLOKOFF-KHNZORIAN (1985); IK89 = IABLOKOFF-KHNZORIAN (1989); J08 = JAKOBSON (1908); 
JH06 = Jaszay \& Hlaváč (2006); Ka40 = Karaman (1940); Ka74 = Karaman (1974); Kh37 = Koch (1937); Ki32 = Kirshenblat (1932); Ki51 = Kirshenblat (1951); Ko46 = Kolenati (1846); Kn90 = Kalashian (1990); L03 = Luze (1903); L05 =Luze (1905); LB04 = LÖBL \& Besuchet (2004); Li71 = LiKovskÝ (1971); Li81 = LiKovskÝ (1981); Lö70 = Löвl (1970); Lu01 = Luze (1901); Lu02 = Luze (1902); Ma06 = MARuYAMA (2006); Mk14 = Makranczy (2014); Mm30 = Mannerheim (1830); Mo60 = Motschulsky (1860); Mü23 = Müller (1923); P67 = Puthz (1967); P72a = Puthz (1972a); P72b = Puthz (1972b); P81 = Puthz (1981); P83 = Puthz (1983); P03 = Puthz (2003); P08 = Puthz (2008); P09 = Puthz (2009); P12 = Puthz (2012); P16 = Puthz (2016); Pa83 = Pace (1983); Pa89 = PACe (1989); Pe80 = Petrenko (1980); Ps06 = PAśnik (2006); R89 = Reitter (1889); R05a = Reitter (1905a); R05b = Reitter (1905b); R08 = Reitter (1908); R09 = ReItTer (1909); Ry1990 = RYVKIn (1990); SBBB04 = SABELLA \& al. (2004); Sc89 = Schülke (1989); Sc04 = Schülke (2004); Sc08 = Schülke (2008); Sc19a = Schülke (2019a); Sc19b = Schülke (2019b); Se03 = SEMENov (2003); SG05 = Solodovnikov \& GrebENnikov (2005); SK00 = SChÜLKE \& Kocian (2000); SKu02 = Sabella \& Kurbatov (2002); Sm04 = SMetana (2004); So02 = SolodovniKov (2002); So04 = Solodovnikov (2004); So05 = Solodovnikov (2005); Sr03 = Schillhammer (2003); Sr04 = SchillhamMER (2004); SS15 = SChülke \& SMEtANA (2015); Sz57 = ScheERPEltz (1957); Sz58 = Scheerpeltz (1958); Sz66a = Scheerpeltz (1966a); Sz66b = Scheerpeltz (1966b); U75 = Ullrich (1975); Uv86 = Ushakov (1986); VB04 = Vít \& Besuchet (2004); Za93 = Zanetti (1993); Za02 = ZANetti (2002).

Footnotes: ${ }^{1)}$ Previous literature records based on erroneously attributing the locality "Araxesthal" (or equivalent) to Armenia rather than Azerbaijan. ${ }^{2)}$ Reported as O. erevanicum Iablokoff-Khnzorian, 1964 (synonym). ${ }^{3)}$ Reported as Mycetoporus ruficornis KRAATZ, 1857 (synonym). ${ }^{4)}$ The record of Mycetoporus brucki (PANDELLÉ, 1869) probably refers to this species. ${ }^{5)}$ The record of Tachinus humeralis humeralis GravenHorst, 1802 probably refers to this subspecies. ${ }^{6)}$ Reported as Lathrimaeum melanochromum IABlokoff-Khnzorian, 1961 (synonym). ${ }^{7)}$ Reported as M. kirschenblatti Iablokoff-Khnzorian, 1956 (synonym). ${ }^{8)}$ Reported as A.crassicornis Iablokoff-Khnzorian, 1835 (synonym). ${ }^{9)}$ Reported as A. nobilis Likovskŕ, 1965 (misidentification). ${ }^{10)}$ The record of A. bobaci KRÁsA, 1931, now a synonym of $A$. parvicornis FAUvel, 1900, most likely refers to the similar A. fugax. ${ }^{11)}$ Reported as Callicerus velox IAblokoff-Khnzorian, 1960 (synonym). ${ }^{12)}$ Reported as Tachinus kirschenblatti Iablokoff-Khnzorian, 1959 (synonym). ${ }^{13)}$ Reported as Pronomaea subterranea IAblokoff-Khnzorian, 1957 (synonym). ${ }^{14)}$ Reported as Atheta nidorum (Thomson, 1868) (synonym). ${ }^{15)}$ Reported as Atheta sipaliformis BeNICK, 1974 (synonym). ${ }^{16)}$ Reported as B. fracticornis (PAYKuLL, 1790) (synonym). ${ }^{17)}$ Locality not specified. ${ }^{18)}$ Reported as S. "cordatoides A". ${ }^{19)}$ Reported as A. filiformis (Latreille, 1806 (synonym). ${ }^{20)}$ Reported as A. silvicola Iablokoff-Khnzorian, 1961 (synonym). ${ }^{21)}$ Partly reported as E. armeniacus CoIfFAIT, 1965, according to Uhlig (pers. comm.) a synonym of E. rivularis. ${ }^{22)}$ Reported as G. latroides Coiffait, 1963 (synonym). ${ }^{23)}$ Reported as G. trossuloides Coiffait, 1966 (synonym). ${ }^{24)}$ Reported as Heterothops flavolimbatus Motschulsky, 1860 (synonym). ${ }^{25)}$ Reported as Ocypus olens (O. MüLler, 1764) (misidentification). ${ }^{25)}$ Reported as O. cyanochloris HochнuтH, 1849 (synonym). ${ }^{26)}$ Reported as O. tomentosus BAUDI, 1870 (synonym). ${ }^{27)}$ Reported as P. lucens (MAnnerheim, 1830) (misidentification). ${ }^{28)}$ Reported as Philonthus armeniacus Bernhauer, 1901 (synonym). ${ }^{29)}$ Reported as P. lutarius (Gravenhorst, 1806) (synonym). ${ }^{30)}$ Reported as S. armeniacus (CoIffait, 1966) (synonym). ${ }^{31)}$ Reported as T. ensifer (J. Müller, 1932) (synonym). ${ }^{32)}$ The record in CoIffait (1972b) is based on the type material from "Araxesthal" and can consequently not be attributed to Armenia. ${ }^{33)}$ Reported as Xantholinus armeniacus CoIffart, 1956 (synonym). ${ }^{34)}$ Reported as Xantholinus microtophilus CoIfFAIt, 1970 (synonym). ${ }^{35)}$ Reported as A. taeniata (Kolenati, 1846) (synonym). ${ }^{36)}$ According to Schülke \& Smetana (2015) currently assigned to Tetralaucopora BernhAUer, 1928, which is most likely a junior synonym of Parocyusa BernHAUER, 1902 (Assing in prep.). ${ }^{37)}$ Reported as B. pubescens (Kolenati, 1846) (synonym). ${ }^{38)}$ Reported as D. canaliculata armeniaca (CAmeron, 1939) (synonym). ${ }^{39)}$ Reported as A. baali Coiffait, 1960 (synonym). ${ }^{40)}$ Reported as M. erevanensis Coiffait, 1970 (synonym) (Assing 2004). ${ }^{41}$ Reported as Medon praecursor Iablokoff-Khnzorian, 1861 (synonym). ${ }^{42)}$ Species dubia. ${ }^{43)}$ Reported as S. armeniacus Coiffait, 1970 (synonym). ${ }^{44)}$ Reported as Scopaeus armeniacus CoIfFAIT, 1968 (synonym). ${ }^{45}$ Reported as Quedius quadripunctatus IABLOKOFF-KHNZORIAN, 1961 (synonym). ${ }^{46)}$ Reported as Quedius acuminatus khnzoriani CoIffait, 1967 (synonym). ${ }^{47)}$ Reported as S. libertas HromádKA, 1990 (synonym). ${ }^{48)}$ Reported as Jurecekia asphaltina (misidentification); see Schillhammer (2004). ${ }^{49)}$ Reported as Paederidus rubrothoracicus caucasicus Scheerpeltz, 1957 (synonym) from “Ost-Armenien”, i.e., not in modern Armenia. ${ }^{50)}$ Record from Armenia in Sm04 and SS15 based on PUTHz (unpublished) (Puthz, pers. comm.); see also additional records section. ${ }^{51)}$ Reported as A. peusi Wagner, 1949 (synonym). ${ }^{52)}$ Distribution extending into North Iran. ${ }^{53)}$ Reported as Zyras stenocephalus IABLOKOFF-Khnzorian, 1960 (synonym). ${ }^{54)}$ Reported as Physetops giganteus Semenov, 1906 (synonym). ${ }^{55)}$ Reported as Aleochara khnzoriani Amiryan, 1999 (synonym). ${ }^{56)}$ Reported as Heterothops armeniacus CoIfFAIT, 1977 (synonym). ${ }^{57)}$ Reported as Heterothops montanus Iablokoff-Khnzorian, 1966 (synonym). ${ }^{58)}$ Reported as Tachyporus armeniacus Kolenati, 1846 (synonym) from "Armeniae montuosae sylvis". ${ }^{59)}$ Previously regarded as subspecies of S. collaris (Müller \& Kunze, 1822). ${ }^{60)}$ Reported as Phyllodrepa armena IAblokoff-Khnzorian, 1959 (synonym). ${ }^{61)}$ Reported as Quedius xanthurus IABLokofF-Khnzorian, 1961 (synonym). ${ }^{62}$ Previously listed as a junior synonym of O. badia. ${ }^{63)}$ Only material identified by Vladimir Gusarov listed; the remainder is given as Mocyta spp. in Tab. 2. 
Mocyta is listed as a subgenus of Atheta in Schülke \& Smetana (2015). However, since V. Gusarov (pers. comm.) is planning to treat this taxon as a distinct genus in his revision (in prep.), this concept is adopted here, too. ${ }^{64)}$ Reported as Tachinus laciniatus Eppelsheim, 1890 (synonym). ${ }^{65)}$ Previously assigned to the genus Enalodroma Thomson, 1859, now a synonym of Alevonota Thomson, 1859 (Assing 2019c). ${ }^{66)}$ Similar to Bisnius zhuk Gusarov, 1995; to be described together with other species of the B. fimetarius group by Schillhammer (in prep.). ${ }^{67)}$ Recorded as Atheta altiviva BENICK, 1974, a name to be synonymized with $A$. araxis by VogEL (in prep.) (VogEL pers. comm.); note that A. araxis is listed in the genus Liogluta Thomson, 1858 by Schülke \& SMetana (2015).

\subsection{Unidentified and unnamed species}

A reliable identification at the species level was not possible for some of the recently collected material because it was represented by females only, because the respective taxon is currently in a state of taxonomic confusion (Mocyta Mulsant \& Rey, 1874, Homoeusa KraAtz, 1856), or for other reasons. Some of the unidentified species may even be undescribed.

Tab. 2: Unidentified and unnamed species. As in Tab. 1, the number of specimens is given in parentheses behind the locality number.

\begin{tabular}{|c|c|}
\hline Species & Localities/samples \\
\hline \multicolumn{2}{|l|}{ Omali ina } \\
\hline Lesteva sp. (female) & $76(1)$ \\
\hline \multicolumn{2}{|l|}{ Proteininae } \\
\hline $\begin{array}{l}\text { Proteinus spp. } \\
\text { (females) }\end{array}$ & $\begin{array}{l}\text { 39(1), 68(1), 114(3), 119(1), } \\
151(3)\end{array}$ \\
\hline \multicolumn{2}{|l|}{ Pselaphinae } \\
\hline Bryaxis spp. & $\begin{array}{l}15(1), \quad 39(1), \quad 52(1), \\
81 \mathrm{a}(2), 88(1), 89(2),\end{array}$ \\
\hline Euplectus sp. & $68 \mathrm{a}(1)$ \\
\hline Tychus spp. & $22(1), 121(1), 121 \mathrm{a}(1)$ \\
\hline \multicolumn{2}{|l|}{ Tachyporinae } \\
\hline Mycetoporus spp. & $\begin{array}{l}7(1), 9(1), 26(1), 30(1), 35(1), \\
36(1), 46(1), 123(1)\end{array}$ \\
\hline \multicolumn{2}{|l|}{ Aleocharinae } \\
\hline Acrotona spp. & $8(1), 127(3)$ \\
\hline Aleochara spp. & $\begin{array}{l}\text { 4(1), 9(6), 13(2), 70(2), 88(1), } \\
155(1)\end{array}$ \\
\hline Aloconota sp. & $135(1)$ \\
\hline Amischa spp. & $46(1), 67(1), 139(1)$ \\
\hline Atheta (Atheta) sp. & $11 \mathrm{~b}(1)$ \\
\hline Atheta (Dimetota) spp. & $11(1), 11 b(1), 13(1)$ \\
\hline Atheta (Dralica) sp. & $19(1)$ \\
\hline $\begin{array}{l}\text { Atheta (Microdota) } \\
\text { spp. }\end{array}$ & $\begin{array}{l}11 \mathrm{a}(1), 59(1), \quad 76(4), \\
114(1), 118(1), 126(1)\end{array}$ \\
\hline $\begin{array}{l}\text { Atheta (Parameotica) } \\
\text { sp. }\end{array}$ & $77(1)$ \\
\hline $\begin{array}{l}\text { Atheta (Philhygra) spp. } \\
\text { (females) }\end{array}$ & $16(2), 67(5), 168(1)$ \\
\hline
\end{tabular}

\begin{tabular}{|c|c|}
\hline Species & Localities/samples \\
\hline Atheta spp. & $\begin{array}{l}9(1), \quad 12(1), \quad 87(1), \quad 112(1), \\
199(1), 199 a(2), 203 a(1)\end{array}$ \\
\hline $\begin{array}{l}\text { Gyrophaena spp. } \\
\text { (females) }\end{array}$ & $\begin{array}{l}30(1), \quad 62(1), \quad 66 a(4), \quad 92(1), \\
102(1), 112(2), 127(2), 131(1)\end{array}$ \\
\hline Homoeusa sp. & $63(1)$ \\
\hline Liogluta sp. & $105(2)$ \\
\hline Mocyta spp. & $\begin{array}{l}1(12), 4(5), 5(2), 6(1), 8(3), \\
9(2), 11(10), 11 \mathrm{a}(6), 11 \mathrm{~b}(31), \\
12(1), 13(1), 16(11), 19(21), \\
20(4), 21(44), 21 \mathrm{a}(1), 22(328), \\
22 \mathrm{a}(97), 26(1), 30(98), 31(8), \\
32(1), 33(2), 34(24), 35(41), \\
36(32), 39(48), 41(19), 42(10), \\
43(5), 45(1), 46(15), 47(5), \\
48(76), 49(9), 50(7), 50 \mathrm{a}(1), \\
53(8), 58(1), 60(2), \quad 61(1), \\
62(2), 63(6), 64(1), \quad 66(4), \\
66 \mathrm{a}(7), 66 \mathrm{~b}(2), 67(71), 68(2), \\
70(35), 71(7), 72(3), 75(1), \\
76(6), 77(5), 78(33), 79(69), \\
81(24), 81 \mathrm{a}(7), 82(22), 82 \mathrm{a}(46), \\
83(9), 85 \mathrm{c}(16), 86(2), 88(33), \\
89(32), 89 \mathrm{~b}(45), 90(1), 90 \mathrm{a}(1), \\
91(4), 92(9), 96(1), 98(8), \\
101(3), 102(8), 103(9), 105(12), \\
107(1), 108(6), 109(1), 110(14), \\
112(12), \quad 113(1), \quad 114(66), \\
115(3), \quad 116(99), \quad 117(1), \\
118(3), \quad 118 \mathrm{a}(1), \quad 119(65), \\
121(1), 121 \mathrm{a}(2), 123(3), 124(1), \\
125(11), \quad 126(95), \quad 127(4), \\
129(1), 130(4), 131(2), 133(9), \\
135(1), \quad 136(13), \quad 148(11), \\
150(2), 151 \mathrm{a}(2), 159(3), 170(3), \\
171(1), 172(1), 186(1), 191 \mathrm{a}(1), \\
196(4), 199(2), 199 \mathrm{a}(1)\end{array}$ \\
\hline Ocalea sp. & $117(1), 121(2)$ \\
\hline Oxypoda spp. & $5(1), 48(1)$ \\
\hline Tachyusa sp. (female) & $161(1)$ \\
\hline \multicolumn{2}{|l|}{ Scaphidiinae } \\
\hline $\begin{array}{l}\text { Scaphisoma cf. agarici- } \\
\text { num (female) }\end{array}$ & $98(1)$ \\
\hline
\end{tabular}




\begin{tabular}{|c|c|}
\hline Species & Localities/samples \\
\hline \multicolumn{2}{|l|}{ Oxytelinae } \\
\hline Anotylus sp. (female) & $\begin{array}{l}31(1), \quad 39(1), \quad 48(1), \\
82(1), 86(1), 182(1)\end{array}$ \\
\hline $\begin{array}{l}\text { Carpelimus (Boopinus) } \\
\text { sp. (female) }\end{array}$ & $212(1)$ \\
\hline $\begin{array}{l}\text { Carpelimus (Trogo- } \\
\text { phloeus) sp. }\end{array}$ & 190(7), 203(1) \\
\hline $\begin{array}{l}\text { Platystethus sp. } \\
\text { (female) }\end{array}$ & 184(1) \\
\hline \multicolumn{2}{|l|}{ Steninae } \\
\hline $\begin{array}{l}\text { Stenus cf. piscator } \\
\text { (female) }\end{array}$ & $199 \mathrm{a}(1)$ \\
\hline \multicolumn{2}{|l|}{ Scydmaeninae } \\
\hline Euconnus spp. & $58(1), 63(2)$ \\
\hline Nanophthalmus sp. & $30(1)$ \\
\hline Neuraphes sp. & $89 b(1), 110(1)$ \\
\hline \multicolumn{2}{|l|}{ Staphylininae } \\
\hline Bisnius sp. (female) & $94(1)$ \\
\hline $\begin{array}{l}\text { Gabrius (nigritulus } \\
\text { group) spp. (females) }\end{array}$ & $\begin{array}{l}19(1), \quad 20(1), \quad 36(2), \quad 82(1), \\
103(1), 114(1), 117(1)\end{array}$ \\
\hline Heterothops sp. & $114(1), 118(1)$ \\
\hline Leptacinus spp. & $177(1), 183(1)$ \\
\hline Quedius sp. (female) & $135(1)$ \\
\hline
\end{tabular}

\subsection{Erroneous and doubtful records}

Numerous species listed for Armenia in recent catalogues (Herman 2001, Schülke \& Smetana 2015, Smetana $2004)$ and/or reported in other works are based on erroneous or doubtful records. They were either wrongly or doubtfully attributed to (modern) Armenia, or they are at least likely to be based on misidentification.

Many of these records are from localities that are not situated in Armenia (any more), but in Azerbaijan, Georgia, or East Turkey (today). Some of these localities have been misinterpreted even in more recent papers. This is particularly true of "Aresch" (or Aresh, or Geok Tapa), which has been interpreted as Verin (Yerevan), but which is in fact a historical fortress built by the Osmanian empire in the 16th century and located near today's Khaldan, Evlakh district, Azerbaijan. Another frequently mis-assigned locality is "Araxesthal" or "Araxestal"; most of the material from this locality was collected near Ordubad (Azerbaijan). Moreover, a number of previous records from Armenia is based on specimens labelled "Armenisches Gebirge" (or similar), which includes the mountain ranges from Georgia and East Turkey to North Iran. It is unclear which of this material originated from the territory of modern Armenia, if any. In fact, it can be inferred from the records of species with more restricted distributions (e.g., Quedius walteri) that they are most likely based on material from East Turkey and/or the Lesser Caucasus in Georgia. Finally, the "Armenia" of $19^{\text {th }}$-century authors (e.g., Носннитн 1849, 1851) included vast territories of what is Georgia, East Turkey, and Azerbaijan today. Consequently, if no localities were specified, such records had to be discarded.

Previous records were considered doubtful owing to unreliable or erroneous identification under the following conditions:

a) a revision of reference material proved the record to be erroneous;

b) records of species with restricted distributions whose presence in Armenia would be unlikely;

c) records of species of taxonomically difficult groups that can be identified only based on an examination of the genitalia (e.g., Acrotona spp., Atheta spp.), which was not common practice at the time of the publication of a record, a criterion particularly applying to numerous records reported by EICHLER (1930);

d) records of species belonging to groups in which additional similar species were described after the publication of a record (e.g., Oxypoda opaca);

e) records of species of taxonomically difficult groups (especially Aleocharinae) whose reliable identification would have required the expertise of a specialist of the respective taxon.

Tab. 3: Species erroneously or doubtfully recorded from Armenia and Nagorno-Karabakh.

\begin{tabular}{|c|c|}
\hline Species & References \\
\hline \multicolumn{2}{|l|}{ Omalinae } \\
\hline $\begin{array}{l}\text { Anthophagus schneideri } \\
\text { EPPELSHEIM, } 1878\end{array}$ & $\begin{array}{l}\text { Ep78 } 27), \mathrm{H}^{27)} \\
\mathrm{Lu} 02^{27)}\end{array}$ \\
\hline $\begin{array}{l}\text { Eusphalerum zolotarevi } \\
\text { (REITTER, 1909) }\end{array}$ & $\mathrm{Am} 00^{1)}$ \\
\hline $\begin{array}{l}\text { Geodromicus globulicollis } \\
\text { MANNERHEIM, } 1830\end{array}$ & L03 $^{1)}$ \\
\hline $\begin{array}{l}\text { Geodromicus luzianus } \\
\text { BordonI, } 1984\end{array}$ & Bo84 2) \\
\hline $\begin{array}{l}\text { Olophrum assimile } \\
\text { (PAYKULL, 1800) }\end{array}$ & IK64a $^{1)}$, ATHW00 \\
\hline $\begin{array}{l}\text { Omalium cribriceps FAUVEL, } \\
1900\end{array}$ & Am00 1) \\
\hline \multicolumn{2}{|l|}{ Proteininae } \\
\hline $\begin{array}{l}\text { Proteinus planicollis REIT- } \\
\text { TER, } 1905\end{array}$ & $\begin{array}{l}\mathrm{R} 05 \mathrm{a}^{3)}, \mathrm{BrS} 10^{3)} \\
\mathrm{Sm04}{ }^{3)}\end{array}$ \\
\hline $\begin{array}{l}\text { Proteinus reflexicollis } \\
\text { REITTER, } 1905\end{array}$ & $\begin{array}{l}\mathrm{R} 05 \mathrm{~b}^{3)}, \mathrm{BrS}_{10}^{3)} \\
\mathrm{Sm04}\end{array}$ \\
\hline \multicolumn{2}{|l|}{ Pselaphinae } \\
\hline $\begin{array}{l}\text { Afropselaphus pentagonus } \\
\text { (SAULCY, 1878) }\end{array}$ & Ka40, LB04 $\left.{ }^{6}\right)$ \\
\hline
\end{tabular}




\begin{tabular}{|c|c|}
\hline Species & References \\
\hline $\begin{array}{l}\text { Batrisus erivanus Мот- } \\
\text { SCHULSKy, } 1845\end{array}$ & $\mathrm{LB}^{21)}$ \\
\hline $\begin{array}{l}\text { Bryaxis crassicornis (Mot- } \\
\text { SCHULSKY, 1835) }\end{array}$ & BK07 15) \\
\hline $\begin{array}{l}\text { Claviger araxidis REITTER, } \\
1890\end{array}$ & $\mathrm{LB}^{3} 4^{3)}$ \\
\hline $\begin{array}{l}\text { Claviger raffrayi REITTER, } \\
1893\end{array}$ & $\mathrm{LB}^{2} 4^{3)}$ \\
\hline $\begin{array}{l}\text { Euplectus thomlini Joy, } \\
1906^{4)}\end{array}$ & E30 \\
\hline $\begin{array}{l}\text { Tychus armeniacus } \\
\text { SAULCY, } 1878\end{array}$ & BS99 \\
\hline $\begin{array}{l}\text { Tychus onobrychidis (IABLO- } \\
\text { KOFF-KHNZORIAN, 1957) }\end{array}$ & IK57c ${ }^{32)}, \mathrm{LB}^{322}$ \\
\hline $\begin{array}{l}\text { Tychus serricornis MoT- } \\
\text { sCHULSKY, } 1845\end{array}$ & $\mathrm{LB}^{21)}$ \\
\hline Tachyporinae & \\
\hline $\begin{array}{l}\text { Ischnosoma myops (EPPELS- } \\
\text { HEIM, 1880) }\end{array}$ & K97 $\left.{ }^{6}\right)$ \\
\hline $\begin{array}{l}\text { Mycetoporus ambiguus LuzE, } \\
1901\end{array}$ & Am00 ${ }^{1)}$ \\
\hline $\begin{array}{l}\text { Mycetoporus baudueri } \\
\text { Mulsant \& Rey, } 1875\end{array}$ & ${\mathrm{Lu} 01^{1)}}^{1)}$ \\
\hline $\begin{array}{l}\text { Sepedophilus marshami } \\
\text { (STEPHENs, 1832) }\end{array}$ & ATHW00 ${ }^{1)}$ \\
\hline $\begin{array}{l}\text { Sepedophilus pedicularius } \\
\text { (GravENHORST, 1802) }\end{array}$ & $\mathrm{E} 30^{1)}$ \\
\hline $\begin{array}{l}\text { Tachinus fauveli PANDELLÉ, } \\
1869\end{array}$ & $\mathrm{LO0}^{6)}$ \\
\hline $\begin{array}{l}\text { Tachinus punctipennis } \\
\text { J. SAHLBERG, } 1876\end{array}$ & U75 ${ }^{6)}$ \\
\hline Aleocharinae & \\
\hline $\begin{array}{l}\text { Acrotona pygmaea (GRAVEN- } \\
\text { HORST, 1802) }\end{array}$ & E30 ${ }^{1)}$ \\
\hline $\begin{array}{l}\text { Aleochara adusta EPPELSHEIM, } \\
1890\end{array}$ & Sm04 ${ }^{1)}$ \\
\hline $\begin{array}{l}\text { Aleochara ignipennis FAUVEL, } \\
1900\end{array}$ & $\operatorname{Li71}{ }^{26)}$ \\
\hline $\begin{array}{l}\text { Aleochara laevigata GYLLEN- } \\
\text { HAL, } 1810\end{array}$ & E30 ${ }^{1)}$ \\
\hline $\begin{array}{l}\text { Aleochara nobilis LıкоvsкÝ, } \\
1965\end{array}$ & $\operatorname{Li}^{8}{ }^{8)}$ \\
\hline $\begin{array}{l}\text { Aleochara parvicornis FAUvEL, } \\
1900\end{array}$ & $\operatorname{Li71}{ }^{34)}$ \\
\hline $\begin{array}{l}\text { Aleochara rambouseki } \\
\text { LIKOvsKÝ, } 1964\end{array}$ & $\operatorname{Li} 81^{7)}, \mathrm{SS} 15^{1)}$ \\
\hline $\begin{array}{l}\text { Aleochara spadicea } \\
\text { (ERICHSON, 1837) }\end{array}$ & ATHW00, Li71 \\
\hline
\end{tabular}

\begin{tabular}{|c|c|}
\hline Species & References \\
\hline $\begin{array}{l}\text { Aleochara spissicornis } \\
\text { ERICHSON, } 1839\end{array}$ & E30 ${ }^{1)}$ \\
\hline $\begin{array}{l}\text { Amarochara forticornis } \\
\text { (LACORDAIRE, 1835) }\end{array}$ & $\mathrm{BrSz} 26^{20)}, \mathrm{Sm} 04^{20)}$ \\
\hline $\begin{array}{l}\text { Anaulacaspis gratilla } \\
\text { (ERICHSON, 1839) }\end{array}$ & Am00 ${ }^{1)}$ \\
\hline $\begin{array}{l}\text { Atheta amicula (STEPHENS, } \\
\text { 1832) }\end{array}$ & E30 ${ }^{1)}$ \\
\hline $\begin{array}{l}\text { Atheta elongatula (GRAVEN- } \\
\text { HORST, 1802) }\end{array}$ & E30 ${ }^{1)}$ \\
\hline $\begin{array}{l}\text { Atheta euryptera (STEPHENS, } \\
\text { 1832) }\end{array}$ & E30 ${ }^{1)}$ \\
\hline $\begin{array}{l}\text { Atheta filicornis EPPELS- } \\
\text { HEIM, } 1890\end{array}$ & Ep90b ${ }^{6)}$ \\
\hline $\begin{array}{l}\text { Atheta orphana (ERICHSON, } \\
\text { 1837) }\end{array}$ & $\mathrm{Hh}^{11)}$ \\
\hline $\begin{array}{l}\text { Brundinia concolor (EPPELS- } \\
\text { HEIM, 1892) }\end{array}$ & $\mathrm{Be} 74^{1)}$ \\
\hline $\begin{array}{l}\text { Euryalea murina (ERICHSON, } \\
\text { 1839) }\end{array}$ & E30 ${ }^{1)}$ \\
\hline $\begin{array}{l}\text { Gyrophaena lucidula ERICH- } \\
\text { sON, } 1837\end{array}$ & $\mathrm{Ko}^{11)}$ \\
\hline $\begin{array}{l}\text { Ischnoglossa prolixa (GRAVEN- } \\
\text { HORST, 1802) }\end{array}$ & Ko46 ${ }^{38)}$ \\
\hline $\begin{array}{l}\text { Leptusa pulchella (MANNER- } \\
\text { HEIM, 1830) }\end{array}$ & $\mathrm{Sm} 04^{17)}$ \\
\hline Oreusa araxis (ReItTER, 1898) & $\mathrm{Sm} 04^{3)}$ \\
\hline $\begin{array}{l}\text { Oxypoda opaca (GRAVEN- } \\
\text { HORST, 1802) }\end{array}$ & E30 ${ }^{1)}$ \\
\hline $\begin{array}{l}\text { Tachyusa balteata ERICHSON, } \\
1839\end{array}$ & E30 35$)$ \\
\hline $\begin{array}{l}\text { Tachyusa coarctata ERICHSON, } \\
1837\end{array}$ & SS15 ${ }^{19)}$ \\
\hline Sc aphidina e & \\
\hline $\begin{array}{l}\text { Scaphisoma laeviusculum } \\
\text { REITTER, } 1898\end{array}$ & Lö70 ${ }^{6)}$ \\
\hline Oxytelinae & \\
\hline $\begin{array}{l}\text { Carpelimus pusillus (GRAVEN- } \\
\text { HORST, 1802) }\end{array}$ & Gi156) \\
\hline $\begin{array}{l}\text { Carpelimus transversicollis } \\
\text { (SCHEERPELTZ, 1947) }\end{array}$ & Gi15 ${ }^{6)}$ \\
\hline $\begin{array}{l}\text { Ochthephilus emarginatus } \\
\text { (FAUVEL, 1871) }\end{array}$ & Mk14 ${ }^{3)}$ \\
\hline $\begin{array}{l}\text { Ochthephilus sericinus (SOLSKY, } \\
\text { 1874) }\end{array}$ & Mk14 ${ }^{3)}$ \\
\hline $\begin{array}{l}\text { Platystethus arenarius } \\
\text { (GEOFFROY, 1785) }\end{array}$ & Am00 1) \\
\hline
\end{tabular}




\begin{tabular}{|c|c|}
\hline Species & References \\
\hline $\begin{array}{l}\text { Platystethus laevis MäRKEL \& } \\
\text { KIESENWETTER, } 1848\end{array}$ & Am00 1) 10) \\
\hline $\begin{array}{l}\text { Platystethus rufospinus } \\
\text { Носннuтн, } 1851\end{array}$ & Hh51 ${ }^{5)}$ \\
\hline Steninae & \\
\hline Stenus aereus Solsky, 1871 & $\mathrm{Am00}{ }^{1)}$ \\
\hline $\begin{array}{l}\text { Stenus arabicus SAULCY, } \\
1865\end{array}$ & $\mathrm{Pu} 72 \mathrm{a}^{9)}$ \\
\hline $\begin{array}{l}\text { Stenus circularis GRAVEN- } \\
\text { HORST, } 1802\end{array}$ & $\mathrm{Sm} 04^{28)}$ \\
\hline $\begin{array}{l}\text { Stenus incanus ERICHsON, } \\
1839\end{array}$ & $\mathrm{Sm} 04^{29)}$ \\
\hline $\begin{array}{l}\text { Stenus melanarius melanarius } \\
\text { STEPHENS, } 1833\end{array}$ & Sm04 ${ }^{30)}$ \\
\hline $\begin{array}{l}\text { Stenus pallitarsis abanticola } \\
\text { Putнz, } 1972\end{array}$ & $\mathrm{Sm} 04^{31)}$ \\
\hline Scydmaeninae & \\
\hline $\begin{array}{l}\text { Euconnus robustus REITTER, } \\
1882\end{array}$ & Ka74 ${ }^{6}$ \\
\hline $\begin{array}{l}\text { Stenichnus pelliceus HoLD- } \\
\text { HAUs, } 1908\end{array}$ & $\mathrm{Da} 04^{1)}$ \\
\hline Paederinae & \\
\hline $\begin{array}{l}\text { Achenium depressum (GRAVEN- } \\
\text { HORST, 1802) }\end{array}$ & SS15 ${ }^{1)}$ \\
\hline $\begin{array}{l}\text { Achenium planum ERICHSON, } \\
1840\end{array}$ & $\mathrm{~A} 10 \mathrm{~b}^{22)}$ \\
\hline $\begin{array}{l}\text { Achenium quadriceps EPPELS- } \\
\text { HEIM, } 1889\end{array}$ & $\mathrm{~A} 10 \mathrm{~b}^{22)}$ \\
\hline $\begin{array}{l}\text { Astenus gracilis (PAYKULL, } \\
\text { 1789) }\end{array}$ & E30 1) 11) \\
\hline $\begin{array}{l}\text { Lathrobium geminum } \\
\text { KRAATZ, } 1857\end{array}$ & $\mathrm{BrS12}{ }^{1)}, \mathrm{Sm} 04^{1)}$ \\
\hline $\begin{array}{l}\text { Lathrobium pallidipenne } \\
\text { Носннитн, } 1851\end{array}$ & Hh515) \\
\hline $\begin{array}{l}\text { Luzea infirma (ERICHSON, } \\
1840 \text { ) }\end{array}$ & $\mathrm{A} 08 \mathrm{~b}^{22(23)}, \mathrm{A}^{20 \mathrm{a}^{22)}}$ \\
\hline $\begin{array}{l}\text { Luzea nigritula (ERICHSON, } \\
1840 \text { ) }\end{array}$ & ATHW00 ${ }^{1)}$ \\
\hline $\begin{array}{l}\text { Luzea rossica (BERNHAUER, } \\
1908 \text { ) }\end{array}$ & $\mathrm{A} 10 \mathrm{a}^{22)}, \mathrm{BrO8}^{22)}$ \\
\hline $\begin{array}{l}\text { Medon apicalis (KRAATZ, } \\
\text { 1857) }\end{array}$ & ATHW00 ${ }^{1)}$ \\
\hline $\begin{array}{l}\text { Medon fusculus (MANNER- } \\
\text { HEIM, 1830) }\end{array}$ & Sm04 ${ }^{1)}$ \\
\hline $\begin{array}{l}\text { Micrillus pallidus (REITTER, } \\
\text { 1887) }\end{array}$ & $\mathrm{A} 08 \mathrm{a}^{22)}, \mathrm{A}^{2} \mathrm{~b}^{22}$ \\
\hline $\begin{array}{l}\text { Micrillus testaceus (ERICH- } \\
\text { sON, 1840) }\end{array}$ & E30 ${ }^{1)}$ \\
\hline
\end{tabular}

\begin{tabular}{|c|c|}
\hline Species & References \\
\hline $\begin{array}{l}\text { Platydomene angusticollis } \\
\text { (LACORDAIRE, 1835) }\end{array}$ & $\mathrm{Sm} 04^{1)}$ \\
\hline $\begin{array}{l}\text { Platydomene bicolor bicolor } \\
\text { (ERICHSON, 1840) }\end{array}$ & $\mathrm{Sm} 04^{1)}$ \\
\hline $\begin{array}{l}\text { Pseudobium angusticolle } \\
\text { (Носннитн, 1851) }\end{array}$ & Hh51 ${ }^{5)}$ \\
\hline $\begin{array}{l}\text { Pseudolathra araxidis CoIF- } \\
\text { FAIT, } 1972\end{array}$ & A17a ${ }^{25)}, C 72 a^{34)}$ \\
\hline $\begin{array}{l}\text { Rugilus angustatus (GEOF- } \\
\text { FROY, 1785) }\end{array}$ & Sm04 ${ }^{1)}$ \\
\hline $\begin{array}{l}\text { Scopaeus minutus ERICH- } \\
\text { son, } 1840\end{array}$ & Am00 1) 36) \\
\hline Staphylininae & \\
\hline $\begin{array}{l}\text { Gabrius astutus (ERICHSON, } \\
\text { 1840) }\end{array}$ & Fa74 ${ }^{1)}, \mathrm{Hh} 1^{5) 24)}$ \\
\hline $\begin{array}{l}\text { Gauropterus sanguinipes } \\
\text { REITTER, } 1889\end{array}$ & $\mathrm{R} 89^{26)}, \mathrm{C} 72 \mathrm{~b}^{26)}$ \\
\hline $\begin{array}{l}\text { Ocypus helleni (J. MüLLER, } \\
\text { 1926) }\end{array}$ & Sz58 ${ }^{14)}$ \\
\hline $\begin{array}{l}\text { Othius hebes Assing \& } \\
\text { SolodovNIKOv, } 1998\end{array}$ & ASo98 ${ }^{6}$ \\
\hline $\begin{array}{l}\text { Philonthus armeniacus } \\
\text { Носннuтн, } 1851\end{array}$ & Hh51 ${ }^{5)}$ \\
\hline $\begin{array}{l}\text { Philonthus rotundicollis } \\
\text { (MÉNÉTRIÉs, 1832) }\end{array}$ & ATHW00 ${ }^{1)}$ \\
\hline $\begin{array}{l}\text { Philonthus salinus KIESEN- } \\
\text { WETTER, } 1844\end{array}$ & Sz58 ${ }^{14)}$ \\
\hline $\begin{array}{l}\text { Quedius walteri KORGE, } \\
1971\end{array}$ & So05 ${ }^{13)}$ \\
\hline $\begin{array}{l}\text { Rabigus formosus (MоT- } \\
\text { sCHULSKY, 1860) }\end{array}$ & $A m 00^{1)}$ \\
\hline $\begin{array}{l}\text { Rabigus tenuis (FABRICIUS, } \\
\text { 1792) }\end{array}$ & $\mathrm{E} 30^{1)}$ \\
\hline $\begin{array}{l}\text { Tasgius winkleri (BERN- } \\
\text { HAUER, 1906) }\end{array}$ & C74 ${ }^{1) 18)}$ \\
\hline $\begin{array}{l}\text { Xantholinus linearis (Olivier, } \\
1795 \text { ) }\end{array}$ & $\mathrm{Sm} 04^{1)}$ \\
\hline $\begin{array}{l}\text { Xantholinus longiventris HeER, } \\
1839\end{array}$ & Sm04 ${ }^{1)}$ \\
\hline $\begin{array}{l}\text { Xantholinus tricolor (FABRI- } \\
\text { CIUS, 1787) }\end{array}$ & E30 ${ }^{1)}$ \\
\hline $\begin{array}{l}\text { Xantholinus variabilis HocH- } \\
\text { HUTH, } 1851\end{array}$ & $\mathrm{C}^{2} \mathrm{~b}^{12)}, \mathrm{Hh} 1^{5)}$ \\
\hline
\end{tabular}

Footnotes: ${ }^{1)}$ Record most likely based on misidentification. ${ }^{2)}$ The type locality "Schawnabad" was erroneously attributed to Armenia rather than Georgia. ${ }^{3)}$ Previous literature records based on incorrectly attributing the type locality "Araxesthal", "Araxestal", or similar to 
Armenia rather than Azerbaijan. ${ }^{4)}$ Manuscript name. ${ }^{5)}$ Record based on type locality "Armenien" in the sense of $1851 .{ }^{6}$ ) The locality "Armenisches Gebirge" (or equivalent) was - most likely erroneously - attributed to Armenia. ${ }^{7)}$ Reported as A. addenda Likovskŕ, 1981 (synonym), whose type locality "Ordubad" was erroneously attributed to Armenia (Assing 2017a). ${ }^{8)}$ Record based on misidentification (Assing 2017a). ${ }^{9}$ Doubtful female-based record. ${ }^{10)}$ Reported as Platystethus cephalotes EPPELSHeim, 1878 (identification doubtful). ${ }^{11)}$ Reported as A. neglectus (MärkeL, 1844) (synonym). 12) Type locality "Helenendorf" erroneously attributed to Armenia. ${ }^{13)}$ Record erroneously attributed to Armenia (Assing 2016d). ${ }^{14)}$ Record from Turkey, erroneously attributed to Armenia. ${ }^{15)} \operatorname{Record}(\mathrm{s})$ from Georgia, erroneously attributed to Armenia. ${ }^{16)}$ Type locality in Georgia, no Armenian localities reported by BESUCHET \& SABELla (1999). ${ }^{17)}$ Record most likely based on misidentification; the species is not indicated from Armenia in PACE (1989). ${ }^{18)}$ Record based exclusively on females. ${ }^{19)}$ Record/identification doubtful; not indicated from Armenia in PAśNIK (2006). ${ }^{20)}$ Record based on misidentification and locality erroneously attributed to Armenia (Assing 2002). ${ }^{21)}$ Species dubia. ${ }^{22)}$ Record from "Aresch" (or equivalent) [= Geok-Tapa; = Agdash in Azerbaijan] erroneously attributed to Armenia. ${ }^{23)}$ Reported as L. caucasica (Luze, 1912) (synonym). ${ }^{24)}$ Reported as Philonthus erythrostomus Hochнuth, 1851 (synonym). ${ }^{25)}$ Type locality "Djoulfa" [= Culfa is in Nakhchivan (Azerbaijan), not in Armenia. ${ }^{26)}$ The record from "Ordubad" erroneously attributed to Armenia. ${ }^{27)}$ Erroneous record from Armenia in SS15 probably based on Ep78 (Elisabetpol) and/or Lu02 (Helenendorf), both in Azerbaijan. ${ }^{28)}$ Confirmed records from Armenia unknown; record possibly referring to Stenus planifrons robustus (PUTHZ, pers. comm.). ${ }^{29)}$ Confirmed records from Armenia unknown (Puthz, pers. comm.). ${ }^{30)}$ Record refers to $S$. peripherus (Puthz, pers. comm.). ${ }^{31)}$ Record most likely refers to $S$. claritarsis (Puthz, pers. comm.). ${ }^{32)}$ Type locality in Nakhchivan (Azerbaijan), not in Armenia. ${ }^{33)}$ Reported as A. peusi WAgner, 1949 (synonym). ${ }^{34)}$ The record of $A$. bobaci (synonym of A. parvicornis) is probably refers to the similar A. fugax. ${ }^{35)}$ Not indicated from Armenia in PAśNIK (2006); record most likely based on misidentified pale-coloured specimens of T. impressa. ${ }^{36)}$ Record probably referring to the similar S. chalcodactylus (FrISCH pers. comm.). ${ }^{37)}$ Most likely misidentified; other species similar to A. spadicea have been described in the meantime (Assing 2009a). ${ }^{38)}$ Reported from Nagorno-Karabakh as I. pubescens (Kolenati, 1846) (synonym).
Tab. 4: Diversity and systematic composition of the faunas of Armenia, Nagorno-Karabakh, and neighbouring countries and regions. The table summarizes total species numbers and the species numbers and proportions of the seven most speciose subfamilies of Armenia (AR), Nagorno-Karabakh (NK), Georgia (GG), the Russian South European Territory (ST), and Iran (IN). The data for GG, ST, and IN are based on SCHÜLKE $\&$ Smetana (2015) and additions up to approximately the end of 2017.

\begin{tabular}{|c|c|c|c|c|c|}
\hline & AR & NK & GG & ST & IN \\
\hline Species total & 675 & 198 & 887 & 1077 & 784 \\
\hline Aleocharinae & 260 & 78 & 224 & 191 & 151 \\
\hline$\%$ & 38.51 & 39.39 & 25.25 & 17.73 & 19.2 \\
\hline Staphylininae & 95 & 30 & 170 & 266 & 141 \\
\hline$\%$ & 14.07 & 15.15 & 19.17 & 24.70 & 17.98 \\
\hline Tachyporinae & 59 & 28 & 71 & 73 & 36 \\
\hline$\%$ & 8.74 & 14.14 & 6.88 & 6.78 & 4.59 \\
\hline Oxytelinae & 58 & 9 & 74 & 120 & 74 \\
\hline$\%$ & 8.59 & 4.55 & 8.34 & 11.14 & 9.44 \\
\hline Steninae & 50 & 12 & 69 & 83 & 66 \\
\hline$\%$ & 7.41 & 6.06 & 7.78 & 7.71 & 8.42 \\
\hline Paederinae & 47 & 8 & 71 & 105 & 135 \\
\hline$\%$ & 6.96 & 4.04 & 8.00 & 9.75 & 17.22 \\
\hline Omaliinae & 43 & 12 & 60 & 73 & 24 \\
\hline$\%$ & 6.37 & 6.06 & 6.76 & 6.78 & 3.06 \\
\hline Pselaphinae & 41 & 7 & 93 & 98 & 57 \\
\hline$\%$ & 6.07 & 3.54 & 10.48 & 9.10 & 7.27 \\
\hline
\end{tabular}

\subsection{Zoogeography}

\subsubsection{Endemism}

Armenia and Nagorno-Karabakh have a remarkably poor endemic staphylinid fauna, especially when compared to West Georgia and Northeast Turkey. Some species have exclusively been reported from Armenia, but their taxonomic and/or zoogeographic status is unclear: Aleochara armeniaca, A. fugax, Aloconota differens, Atheta erevanensis, Gnypeta gracilior, Hydrosmecta salsolae, Bisnius microtophilus, Gabrius armeniacus, Heterothops macrops, H. microtophilus, Leptacinus armeniacus, Quedius latus, Q. transcaucasicus, Stenistoderus nanus. Similarly, several species have only been reported from Armenia or Nagorno-Karabakh, but may also be present in neighbouring countries: Geodromicus armeniacus, G. rivularis, Olophrum aragatzense, Omalium kociani, Zibus yunonae, Mycetoporus praetextoides, Aleochara polychroma, Atheta hamulata, A. senticollis, Atheta (Oreostiba) abscisa, A. (O.) brevitheca, A. (O.) semialba, Calodera alticola, Liogluta armeniaca, Myllaena 

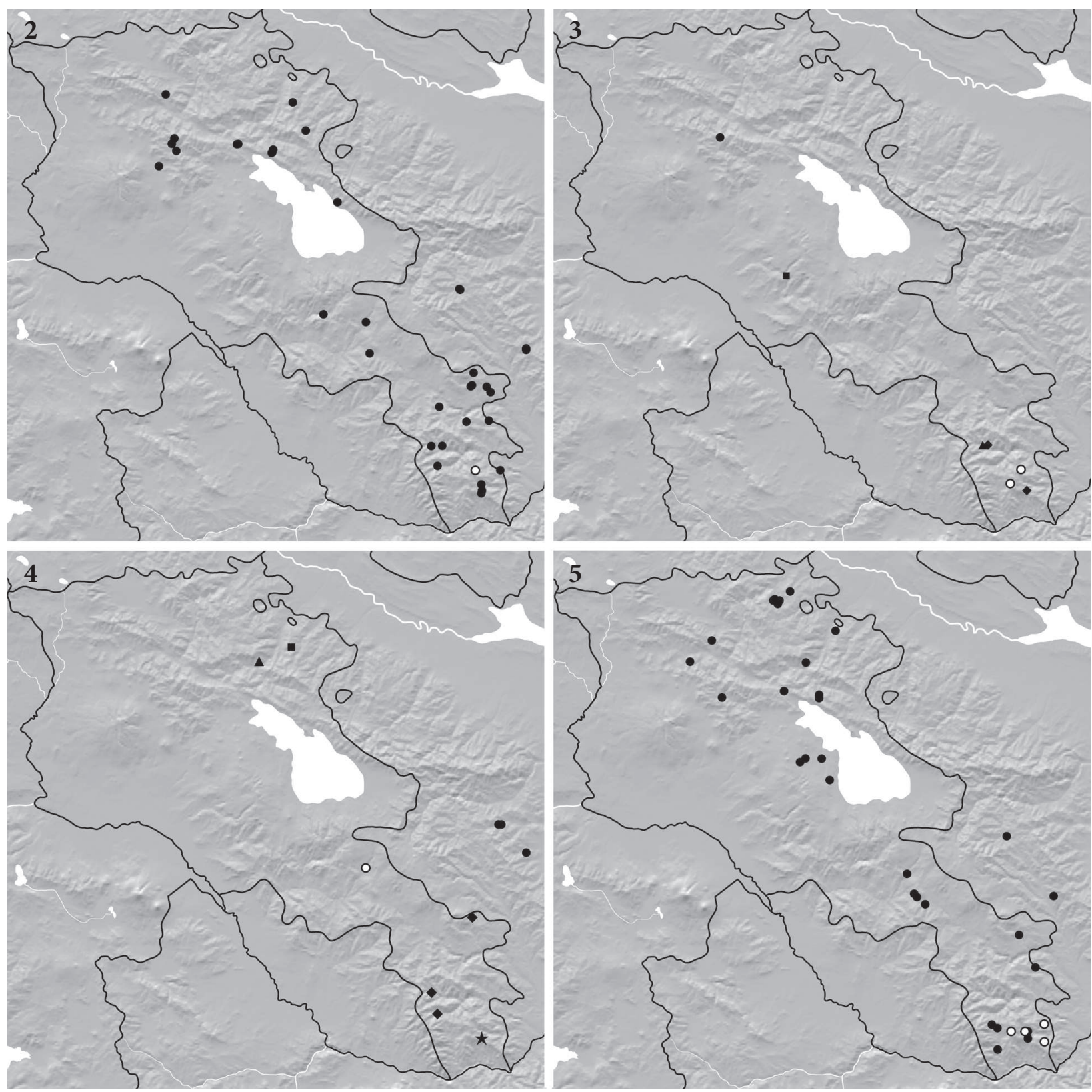

Maps 2-5: Distributions of endemic species and species groups. Map 2 (upper left): regionally endemic Geostiba species (all species pooled; G. sororcula omitted) (black circles) and the locally endemic Bellatheta khustupica (white circle). Map 3 (upper right): Atheta meghruica (white symbols) and regionally endemic Pselaphinae (black symbols): Bryaxis armeniacus (black circle), B. meghruicus (black triangle), B. seductus (black diamonds), Tychus milvus (black square). Map 4 (lower left): Oxypoda grandecristata (white circle) and regionally endemic Scydmaeninae (black symbols): Euconnus karabakhus (black circles), E. longilaminatus (black square), E. tavushus (black triangle), Neuraphes gomarantsus (black star), N. syunikus (black diamonds). Map 5 (lower right): Xantholinus adustus (white circles), a regional endemic of South Armenia, and X. kirschenblati (black circles), a species currently known only from Armenia and Nagorno-Karabakh, but probably present also in adjacent regions.

ambulans, Oxypoda flexa, O. infissoides, O. levipunctata, O. verminata, Anotylus hamatoides, Lobrathium ancoriferum, Medon sequax, and Ontholestes chalcopygus. These species do not possess typical adaptations of endemics (flightlessness, reduced eye size, etc.) and belong to species groups or ecological guilds whose representatives are usually more widespread.

Two of the endemics of South Armenia are relatively common and widespread in this region: Leptusa armeniaca (wingless) and Oxypoda subplicata (winged). They are (L. armeniaca), or may potentially be (O. subplicata) present also in adjacent regions of North Iran.

Regionally endemic species of Armenia and NagornoKarabakh, with one exception (Atheta meghruica) all flightless, belong to the Aleocharinae (twelve species), Pselaphinae (four species), Scydmaeninae (five species), and Staphylininae (one species). These include Atheta (Paralpinia) meghruica (Map 3), Geostiba (Sibiota) deliqua, G. (S.) immutata, G. (S.) kalavanica, G. (S.) meghruica, G. (S.) nigrohortensia, G. (S.) pamba- 
kica, G. (S.) tigrani, G. (S.) unicuneata, G. (S.) unituber, G. (Tropogastrosipalia) khnzoriani (Map 2), and Oxypoda grandecristata (Map 5) of the Aleocharinae, Bryaxis armeniacus, B. meghruicus, B. seductus, and Tychus milvus of the Pselaphinae (Map 3), Euconnus longilaminatus, E. tavushus, E. karabakhus, Neuraphes gomarantsus, and N. syunikus of the Scydmaeninae (Map 4), and Xantholinus adustus (Map 5) of the Staphylininae. Geostiba nigrohortensia and Euconnus karabkhus (Map 4) are endemic to Nagorno-Karabakh, Geostiba unicuneata to adjacent regions in Armenia and Nagorno-Karabakh, and the remainder to Armenia. For maps illustrating the individual distributions of the Geostiba, Leptusa, and Oxypoda species see Assing (2016b, c, 2017b, c, 2018c, h).

Remarkably, the study region is nearly devoid of highaltitude endemics. The only mountain where endemic species associated with habitats above the tree-line were found is Mount Khustup in South Armenia. Based on currently available evidence, one of them, Bellatheta khustupica, may actually be locally endemic (Map 2).

The majority of the regional endemics inhabits montane forests. The greatest density of these species was observed in South Armenia.

\subsubsection{Other distribution types}

The zoogeography of most Staphylinidae species still requires thorough study; many of them are known only from few records. Nevertheless, an analysis of the distributions of the taxa known from Armenia and Nagorno-Karabakh reveals that the majority of the species is widespread. They represent Cosmopolitan, transPalaearctic, expansive Holo- or Ponto-Mediterranean, or other elements. In addition, the fauna of the study region is characterized by the presence of the following distribution types.

Species such as Mycetoporus praetextoides, Xantholinus kirschenblati (Map 5), and Rugilus armeniacus are currently known only from Armenia, Nagorno-Karabakh, and (only the latter) from Azerbaijan. The former two are widespread and not uncommon in Armenia and Karabakh and only few records are known of $R$. armeniacus. It appears likely that these species will eventually be recorded also from Georgia and/or North Iran.

The known distributions of another set of species is restricted to Northeast Turkey, Armenia, and partly also Nagorno-Karabakh. Examples are Proteinus baculatus, Mycetoporus phaedrus, Geostiba sororcula (see Assing 2017b: map 1), Dianous ponticus, and Quedius brevalatus.

A significant proportion of the fauna of Armenia and Nagorno-Karabakh is of the Caspian distribution type, with a refugial centre in Transcaucasia (see LATTIN 1967). Most of these species have a Caucasian distribution typically including the Greater Caucasus, the Lesser Caucasus, the East Pontic Mountains, Transcaucasia, and North Iran, or parts of this region. Representatives of this distribu- tion type are Anthobium hamatum, A. tenue, Dropephylla elisabethae, Eusphalerum fidele, E. sareptanum, E. transcaucasicum, Geodromicus brevicollis, G. major, Olophrum caucasicum, Brachygluta araxidis, B. nodosa, Bryaxis clavipes, B. longipalpis, B. rostratus, Ctenistes marthae, Pselaphus caspicus, Tychus colchicus, T. dubius, T. guillebeaui, Lordithon rostratus, Mycetoporus monticoloides, Sepedophilus transcaucasicus, Tachyporus fascipennis, Tachinus caucasicus, T. cingulatus, T. nigerrimus, Aleochara conviva, A. plicelytrata, A. subtumida, Anaulacaspis caucasica, Atheta altiviva, A. bispinosa, A. brevapicalis, A. chefsurica, A. laevigata, Cousya schuelkei (Assing 2018e: Fig. 142), Gyrophaena caucasica, Leptusa fuliginosa, L. venusta, Myllaena caucasica, Ocalea alutacea, O. dubia, O. minor, Oxypoda articollis, O. caucasica, Pella laeviceps, Tropimenelytron mirabile, Anotylus strigifrons, Platystethus gildenkovi, Thinodromus dilaticollis, T. motschulskyi, Stenus araxis, S. armeniacus, S. caspius, S. medus, S. peripherus, S. skoraszewskyi, Cephennium perispinctum perispinctum, Euconnus lalvarensis, Scydmoraphes yermolowi, Lathrobium permutatum, L. tichomirovae, Leptusa laeviuscula, Ochthephilum egregium, Rugilus longicollis, Scopaeus chalcodactylus, S. khnzoriani, Sunius fulgocephalus, S. khnzoriani, Tetartopeus stylifer, Bisnius pentheri, B. reitteri, Erichsonius dux, Gabrius sacerdotalis, G. trossuliformis, Ocypus forficularius, O. hochhuthi, Othius grandis, O. stenocephalus, Philonthus picimanus, P. svanetiensis, Quedius orientalis, Q. vulneratus, Tasgius eppelsheimianus, T. gracilicornis, T. limbifrons, Xantholinus araxis, and $X$. khnzoriani.

Iranian elements are typically characterized by an often more or less pronounced circum-Caspian distribution eastwards reaching into Middle Asia. The following species may be assigned to this type: Dropephylla caucasica, Batrisodes ruprechtii, Brachygluta spinicoxis spinicoxis, Trissemus melinus, T. montanus, Tychus lederi, T. remaudierei, Anotylus khachikovi, Stenus machulkai, S. piscator, S. turk, Bisnius piochardi, Gabrius muelleri, Philonthus velatipennis, Physetops tataricus, Quedius cohaesus, Xantholinus fortepunctatus.

The Syrian distribution type may be represented in the study region by species such as Cousya araxis (Assing 2018e: figure 141).

The distinction between Iranian, Caspian, and Syrian elements, however, is sometimes difficult and requires a profound knowledge of the distribution. Examples of doubtful cases are Atheta brevapicalis (Iranian or Caspian; see map 1 in Assing (2018b)), Gyrophaena korbi (Iranian or Caspian), Medon fusculoides and M. paradisiacus (Iranian or Caspian), Myrmoecia urartu (Iranian or Syrian; see map 1 in Assing (2019a)), Stenus machulkai (Caspian, Syrian, or Iranian).

A similar problem is encountered with species like Tachinus schneideri and Gyrophaena orientalis, which are distributed in the North Palaearctic and in the Caucasus region. They may represent Siberian elements, but too little is known about their distribution in the Northeast Palaearctic region to be certain. 


\subsubsection{Discontinuous distributions}

Several new records of species from Armenia and Nagorno-Karabakh revealed some remarkable range disjunctions. In rarely recorded and poorly known species pronounced distribution gaps may represent artefacts reflecting the lack of data, as is probably the case with, for instance, Aleochara falcata, a species currently known only from the Russian Central European territory and Armenia. In general, such discontinuous distributions were observed particularly in species found at high altitudes.

Omalium wunderlei was described based on five specimens from three localities (one of them vague) in Southwest Turkey (Antalya) (Zanetri 2002). The species was subsequently reported also from the Turkish province Muğla (Assıng 2004b). In summer 2018, however, a number of specimens was discovered near the peak of Mount Khustup in South Armenia at an altitude of approximately $3,000 \mathrm{~m}$, about $1,500 \mathrm{~km}$ from the previously known range (Map 6).

Similar, though less spectacular examples of species previously known from Turkey, partly in West Turkey, with pronounced gaps between the Turkish localities and those in Armenia and Nagorno-Karabakh are Acrotona concamerata, Atheta albomontis, A. dissimulans, A. lyciana, A. monstruosa, and Oxypoda recta. For a map illustrating the currently known distribution of $O$. recta see Assing (2018c: map 2).

Some disjunctions, however, are even more pronounced. The previously known distribution of Atheta malleiformis was confined to the Alps and adjacent regions and separated from the record in Nagorno-Karabakh by a distance of some $2,700 \mathrm{~km}$. Atheta pechlaneri, $A$. serrata, and $A$. taxiceroides had been recorded only from West, Central, and North Europe (A taxiceroides also from the Italian Apennines; see Tagliapietra \& ZANETti 2003), as well as from Bulgaria; the distance between the Bulgarian records and those from Armenia amounts to more than $1,600 \mathrm{~km}$. Bellatheta palata is currently known only from the Balkans and Armenia, Ischnoglossa obscura from North and Central Europe, Turkey, and Armenia, Oxypoda lentula from Europe, Siberia, Ukraine, and from Armenian localities at altitudes of approximately $3,000 \mathrm{~m}$, Gabrius robustus was previously recorded only from few mountains in Macedonia and Turkey and is now known also from Armenia, with most of the Armenian records at altitudes of approximately $3,000 \mathrm{~m}$. Another spectacular example of a huge distribution gap is Oxypoda spaethi, a nidicolous species associated with gopher burrows (previously known from Austria and Hungary (Assing 2012); gap distance 2,200 m) (Map 7). Based on the maps and concepts provided by LATTIN (1967), this species may represent a Caspian element with postglacial range disjunction.
In the case of Pronomaea flavirostris, the gap is not between the West or Northwest and the study region, but between Middle Asia (Tajikistan, Afghanistan) and Armenia, a distance of approximately $1,700 \mathrm{~km}$ (Map 8).

\section{Ecology}

Staphylinidae are associated with an enormous variety of terrestrial and semiaquatic habitats and can be found practically everywhere. They represent the full range between extreme specialization and extreme generalization. The field trips conducted by the authors were primarily targeted at the native and endemic fauna of natural and semi-natural habitats at intermediate to high altitudes (forests, wetlands, alpine habitats). The fauna associated with such habitats, including the endemic fauna, can now be considered relatively well studied, at least as far as Armenia is concerned. Sampling in Nagorno-Karabakh was confined to a relatively short period in only one year and almost exclusively focused on forests; high-altitude environments were inaccessible and habitats such as decaying dung, river banks, and other wetlands were sampled only rarely. Remarkably, the Armenian fauna above the tree-line is largely composed of widespread species. The only true local endemic in such an environment is Bellatheta khustupica in Mount Khustup, South Armenia.

Habitats that may host numerous additional species to be recorded from the study region are, above all, various kind of decaying matter (dung, compost, rotting wood, bark, carrion, etc.), nests of mammals, birds, and ants, banks of rivers and streams, and other wetlands. Species associated with such habitats are still poorly represented in the fauna of Armenia and Nagorno-Karabakh. For instance, not a single species of Thinobius Kiesenwetter, 1844 (mostly ripicolous), Platydomene Ganglbauer, 1895 (ripicolous), only one of doubtful status of Hydrosmecta THOMson, 1858 (mostly ripicolous), and relatively few of Carpelimus LEACH, 1819 (various wetlands) are known from the region.

Sampling subterranean, endogean or hypogean, fauna has not been attempted. Based on experiences with such a fauna in the East Mediterranean, it does not seem unlikely that at least endogean fauna may be present particularly in South Armenia.

The primary methods used for recording species during the authors' field trips were sifting, floating (river banks), and turning stones (at high altitudes, mostly near snowfields). Applying other methods such as pitfall traps and especially car-netting and window traps (for flying insects) would undoubtedly result in recording species whose habitats are essentially unknown (of which there are numerous examples in Staphylinidae) and in significantly increasing species numbers in the study region. 


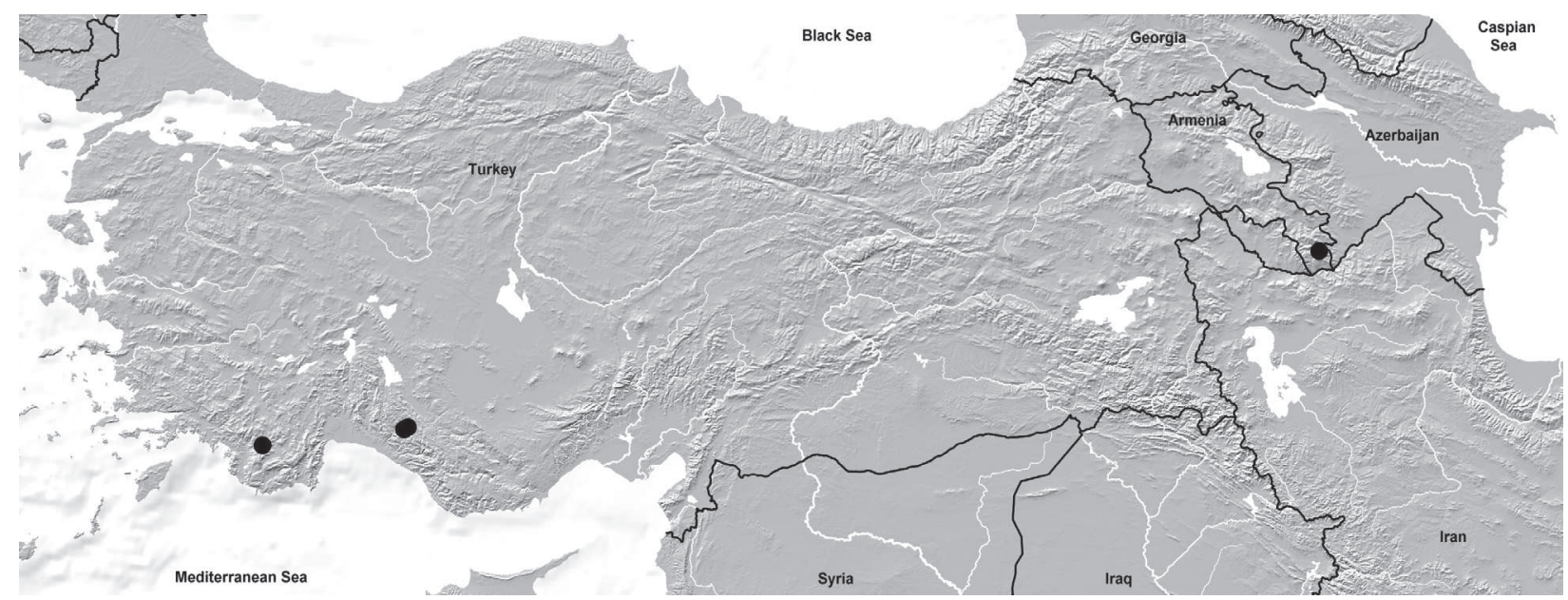

Map 6: Distribution of Omalium wunderlei.

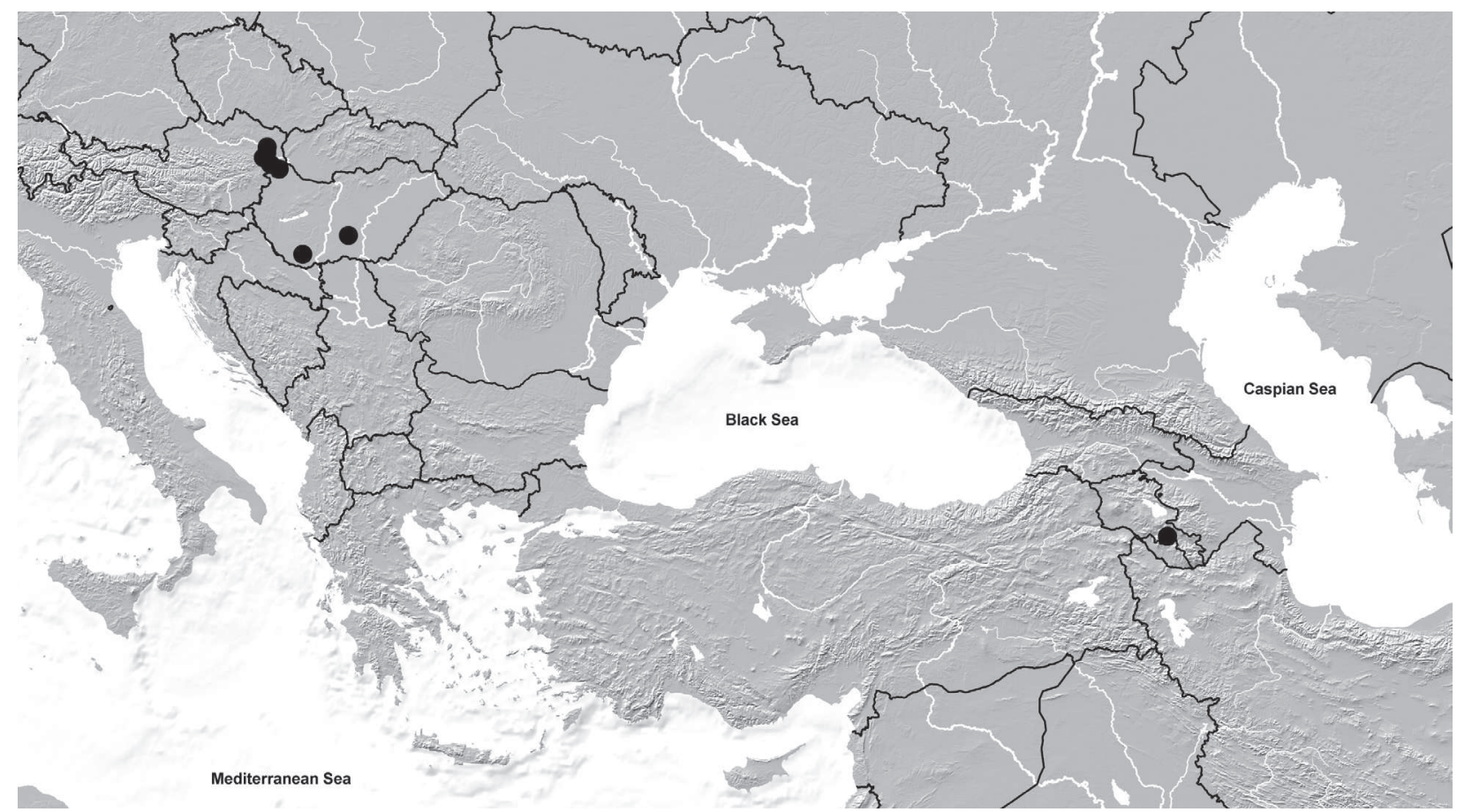

Map 7: Distribution of Oxypoda spaethi (records based on material in cAss and on HorIon (1967)).

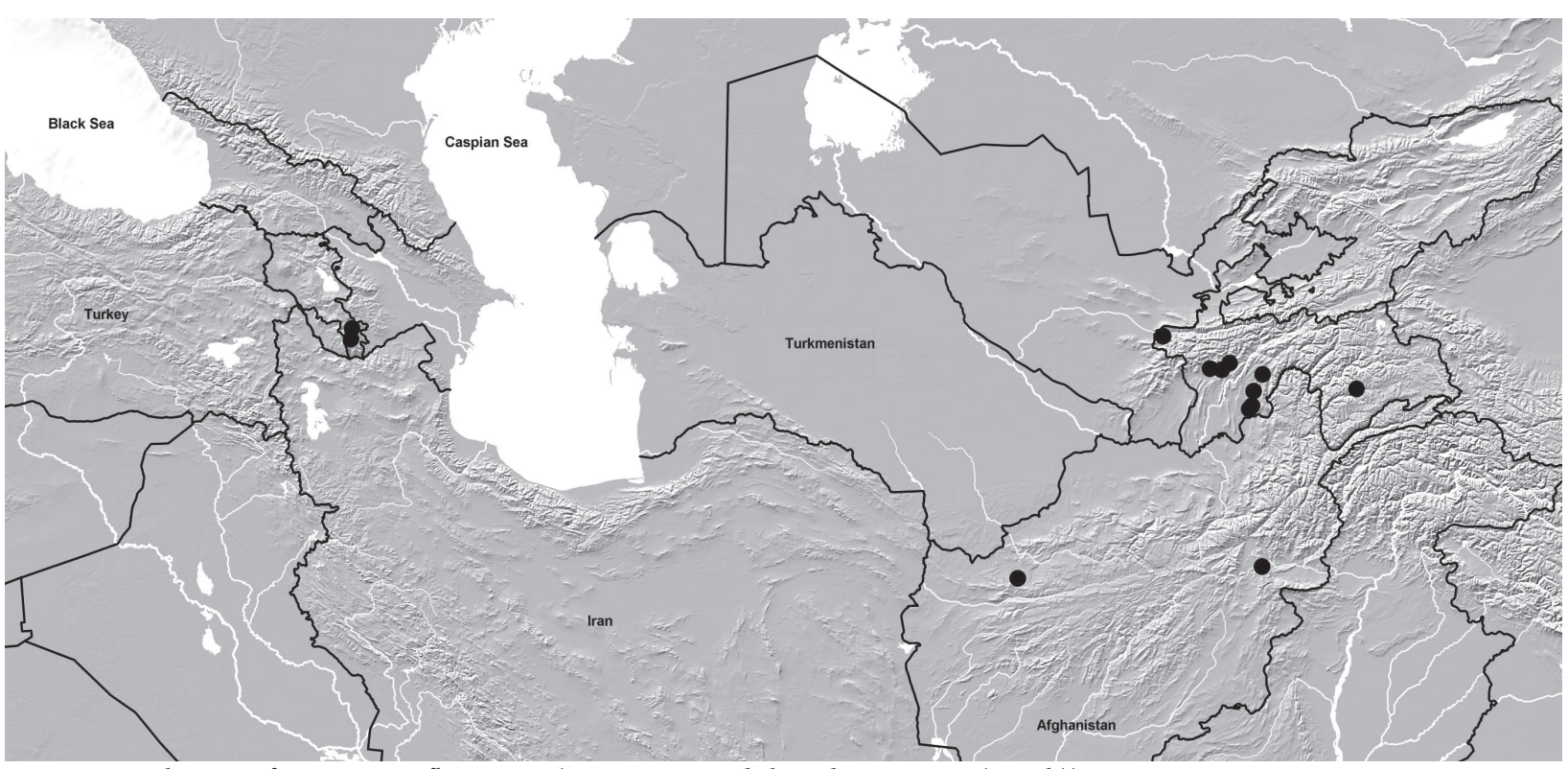

Map 8: Distribution of Pronomaea flavirostris (previous records based on Assing (2007b)). 


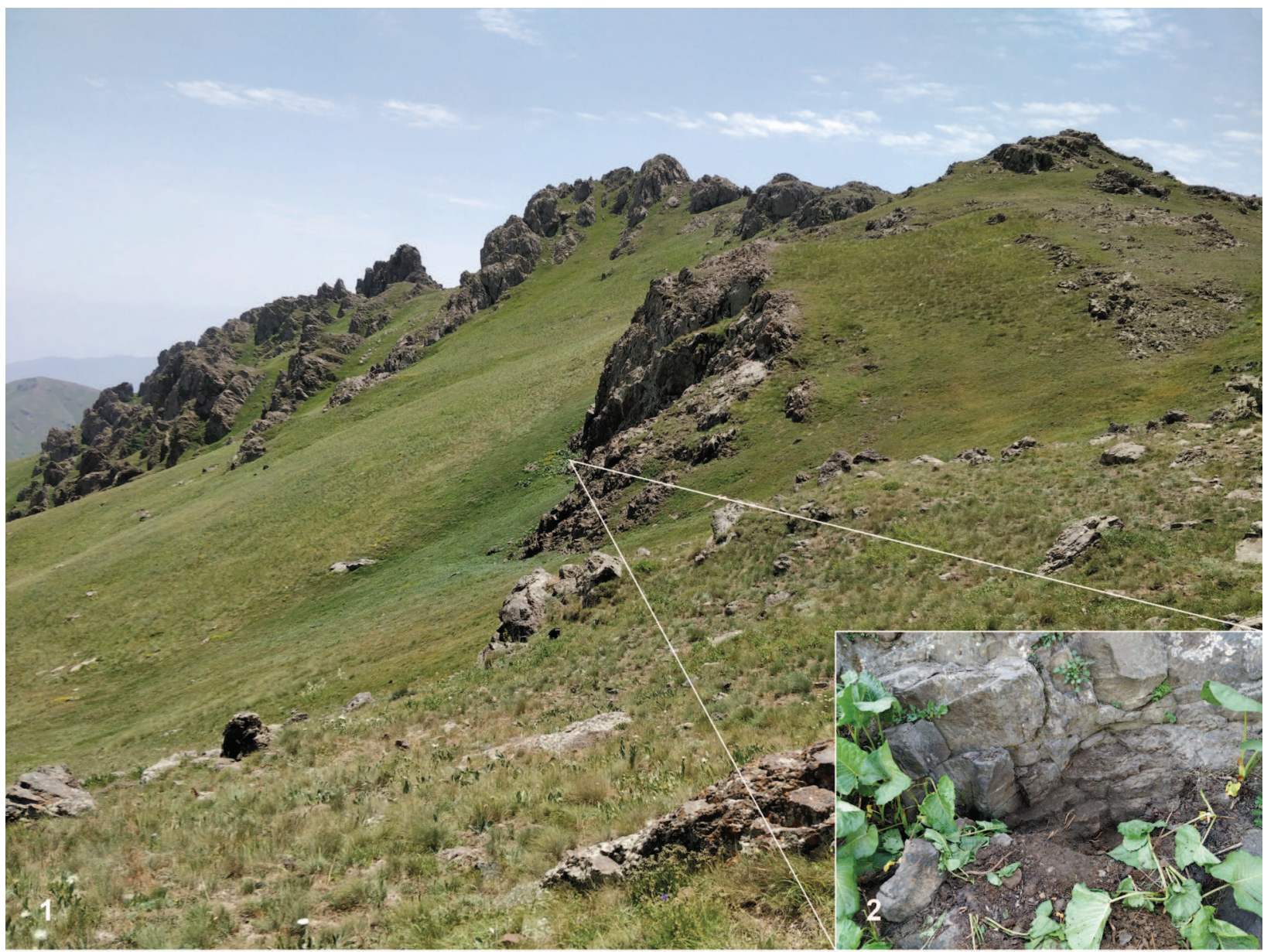

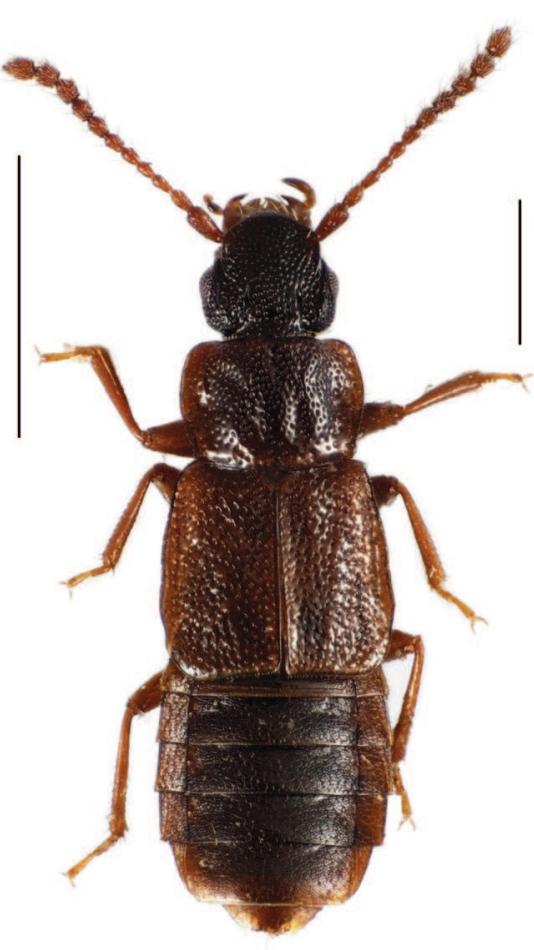

3

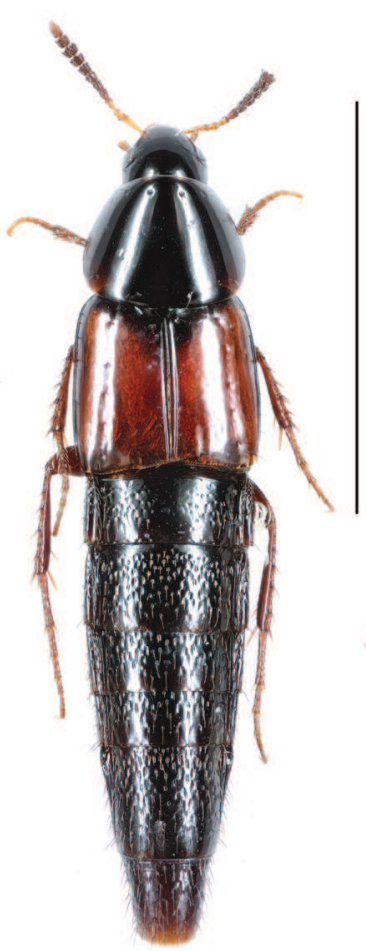

4

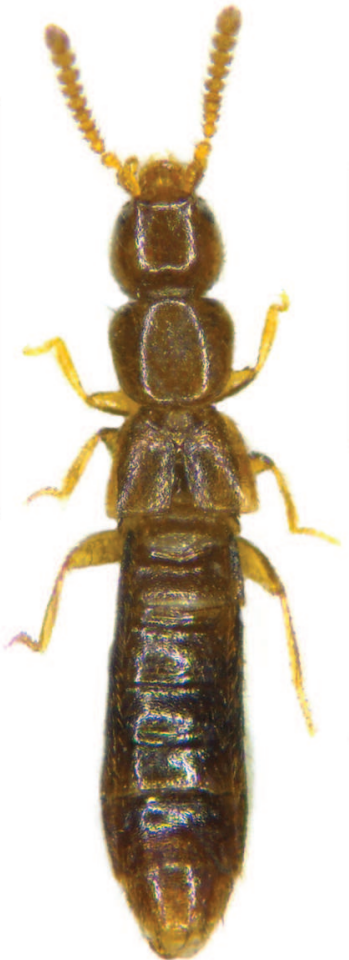

5

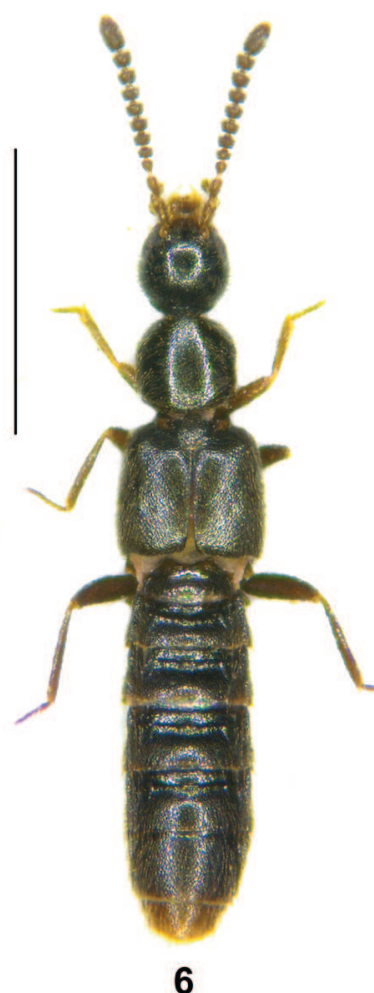

Figs 1-6: Type locality of Bellatheta khustupica in the peak region of Mount Khustup (1); microhabitat where B. khustupica was collected, together with Omalium wunderlei, Anthobium fusculum, and other Staphylinidae (2); habitus of Omalium kociani (3), Mycetoporus silvaticus, holotype (4), Bellatheta khustupica (5), and Calodera alticola (6). Scale bars: $1.0 \mathrm{~mm}$. 


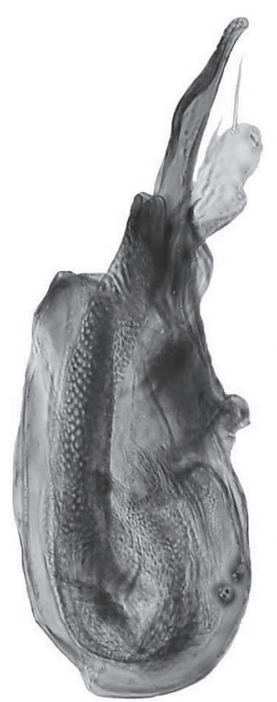

7
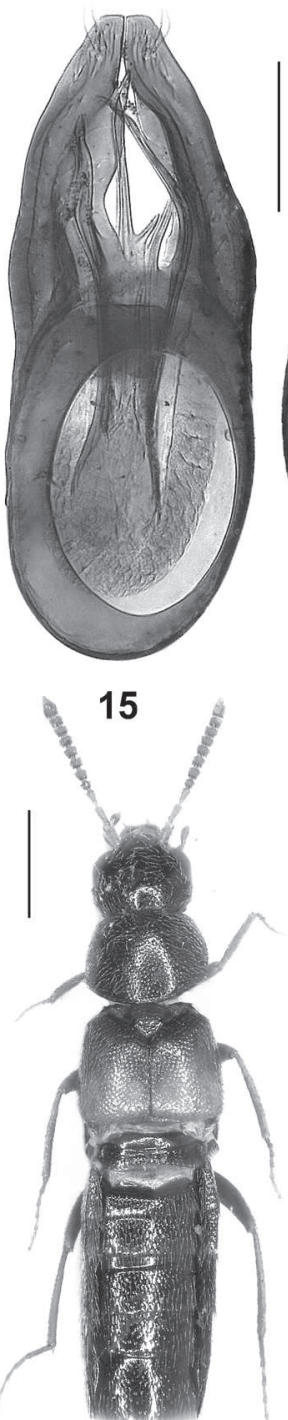

19

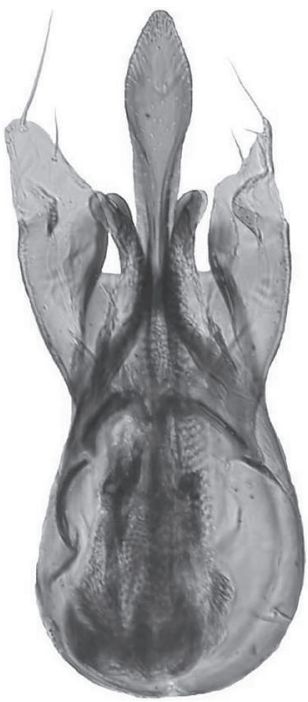

8

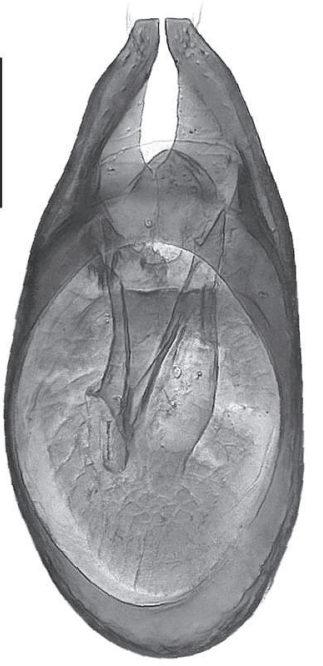

16

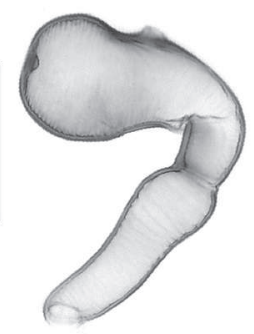

21

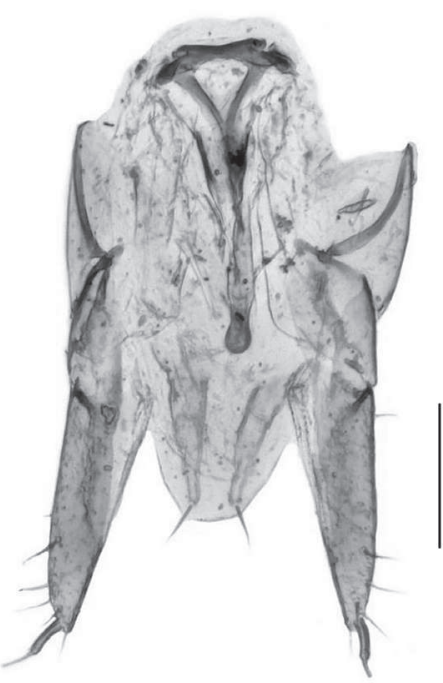

9

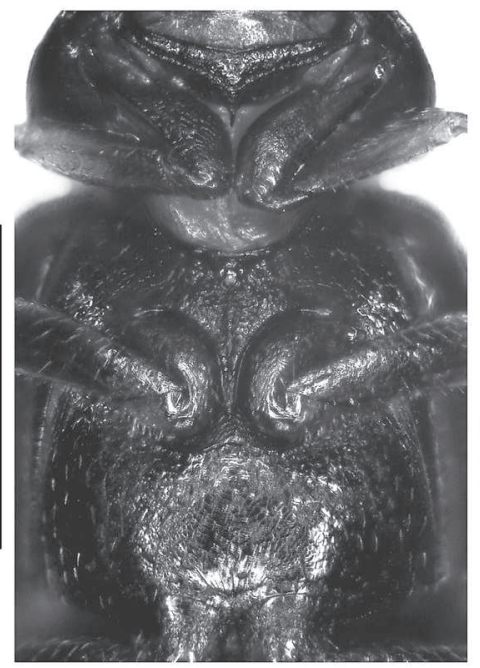

12

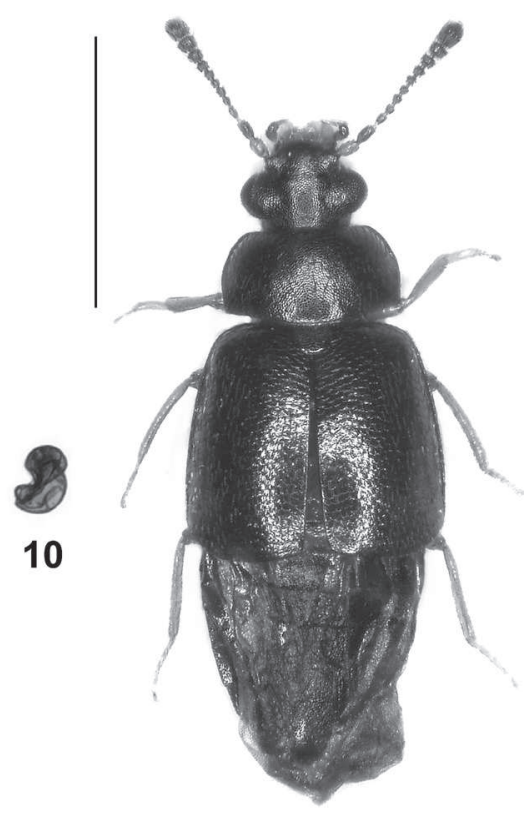

11

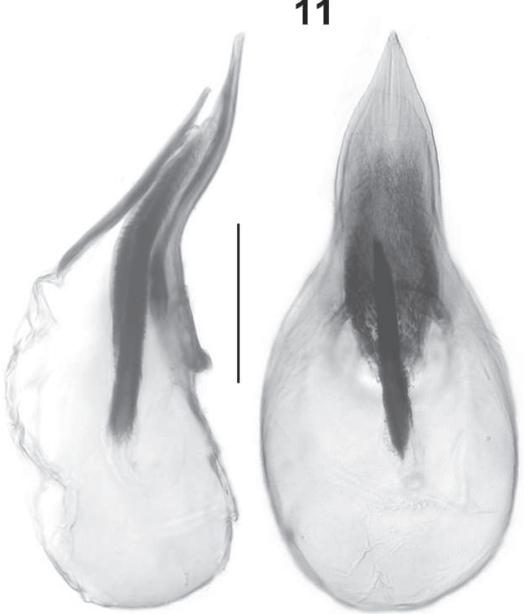

13

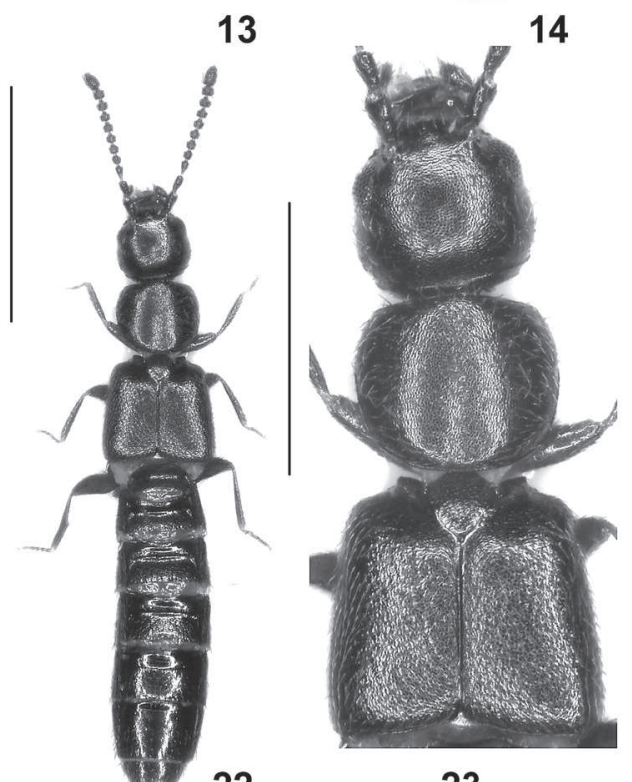

14

22

23

Figs 7-23: Omalium kociani (7-10), Proteinus baculatus (11-14), Bryaxis armeniacus (15, 17), B. meghruicus (16, 18), Aleochara fugax (19-21), and Atheta meghruica (22-23): (median lobe of) aedeagus in lateral and in ventral view (7-8, 13-16); female accessory sclerites $(9)$; spermatheca $(10,21)$; habitus $(11,19,22)$; thorax in ventral view $(12)$; male antennomeres I-III (17-18); posterior margin of female sternite VIII (20); forebody (23). Scale bars: 11, 19, 22: $1.0 \mathrm{~mm}$; 12, 23: $0.5 \mathrm{~mm}$; 7-10, 13-16, 20-21: $0.1 \mathrm{~mm} ; 17-18$ : without scale. 


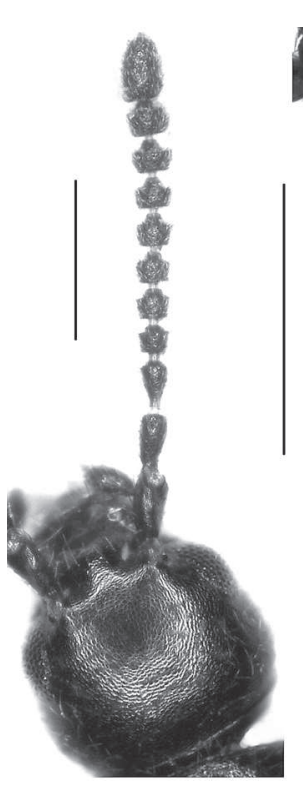

24

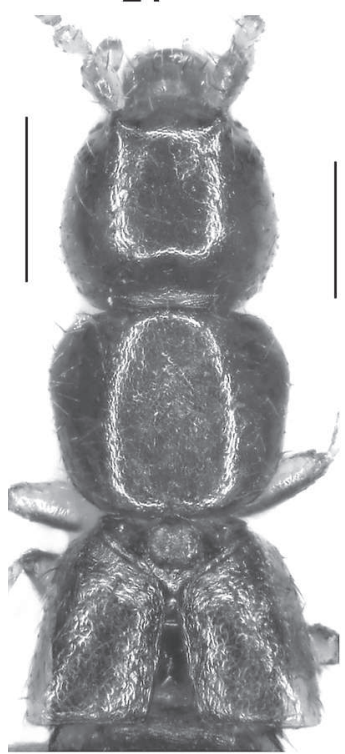

29

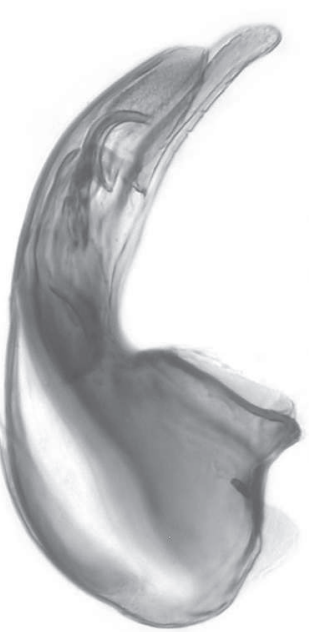

31

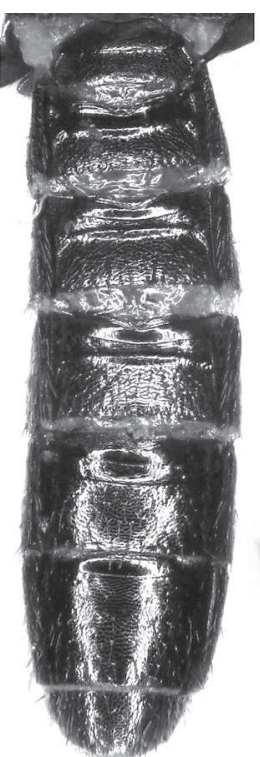

25

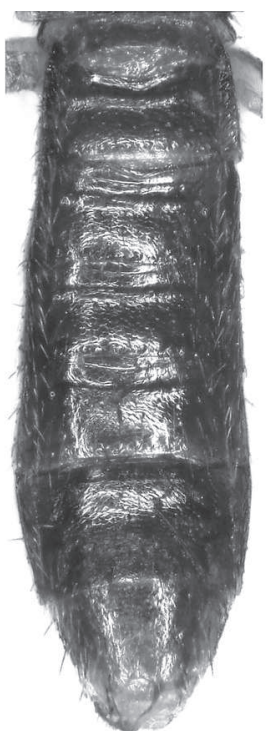

30

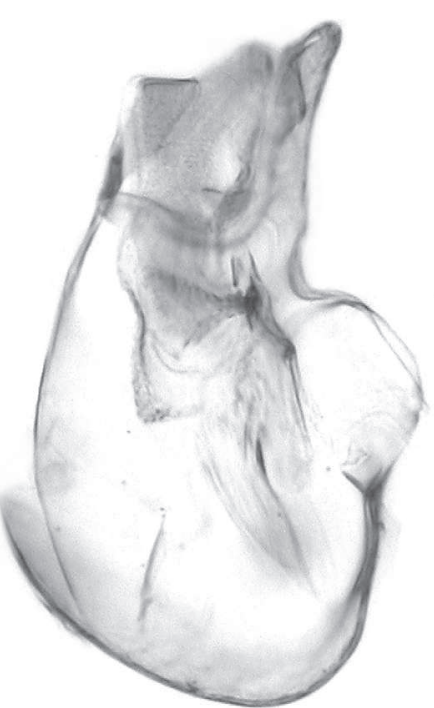

26

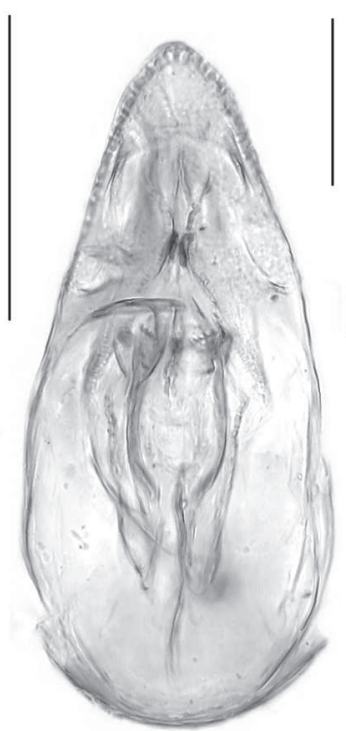

27

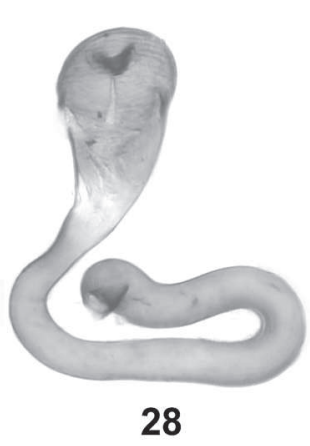

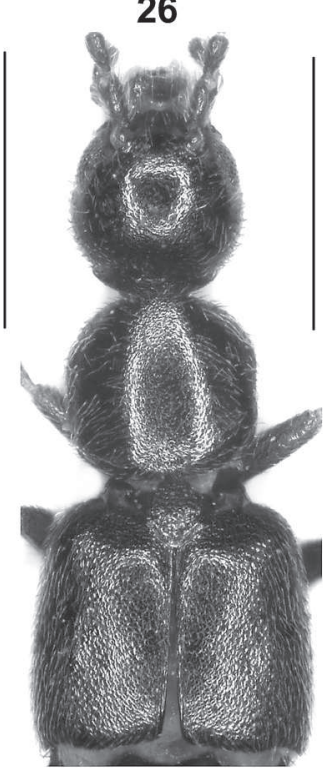

36

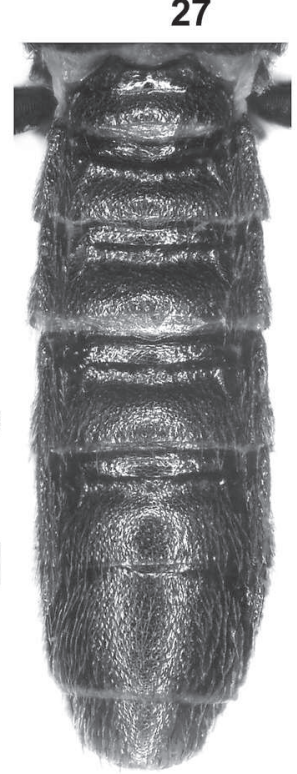

37

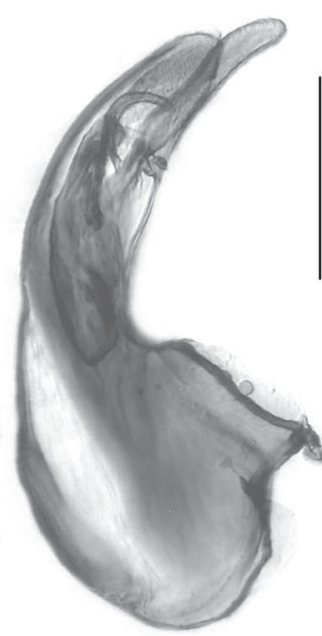

32

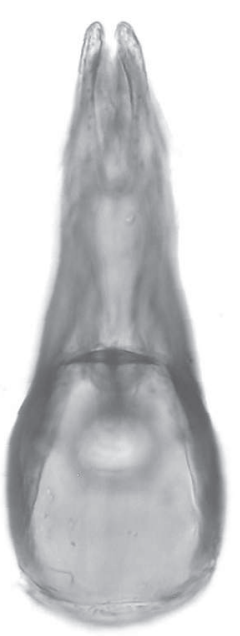

33

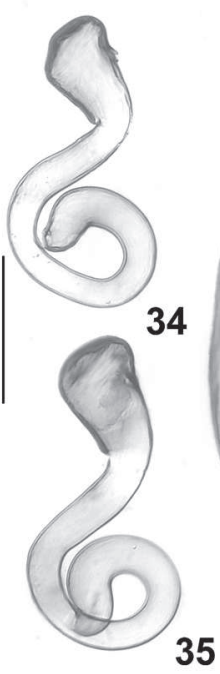

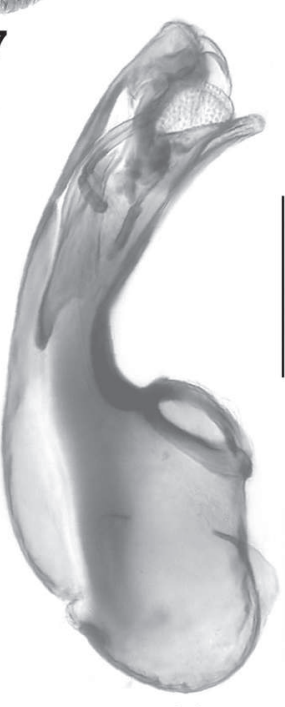

38

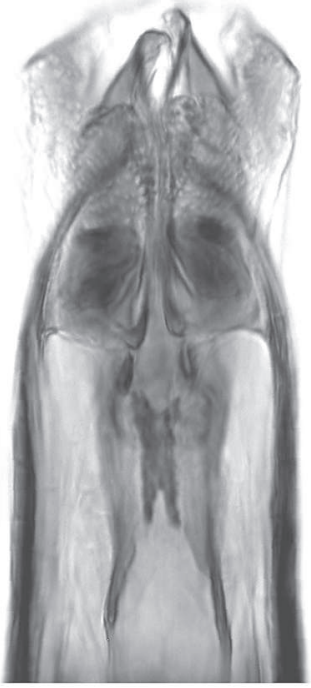

40

Figs 24-40: Atheta meghruica (24-28), Bellatheta khustupica (29-35), and Calodera alticola (36-40): head (24); abdomen (25, 30, 37 ); median lobe of aedeagus in lateral and in ventral view (26-27, 31-33, 38-39); spermatheca (28, 34-35); forebody (29, 36); apical portion of median lobe of aedeagus in ventral view (40). Scale bars: $25,36-37: 0.5 \mathrm{~mm} ; 24,29-30: 0.2 \mathrm{~mm}$; 26-28, 31-35, 38-40: $0.1 \mathrm{~mm}$. 


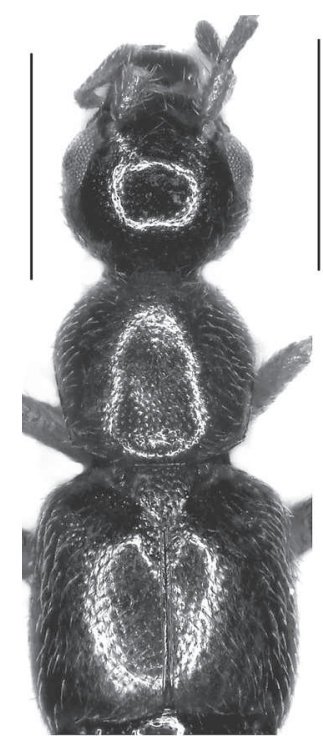

41

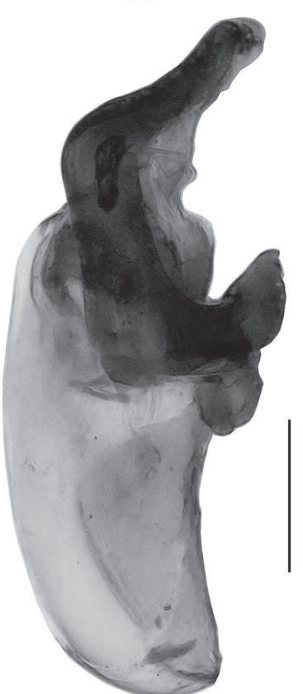

51
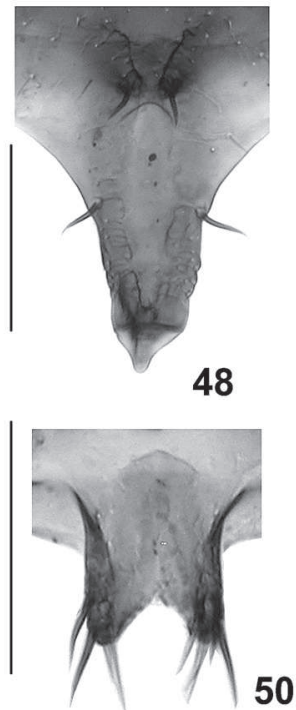

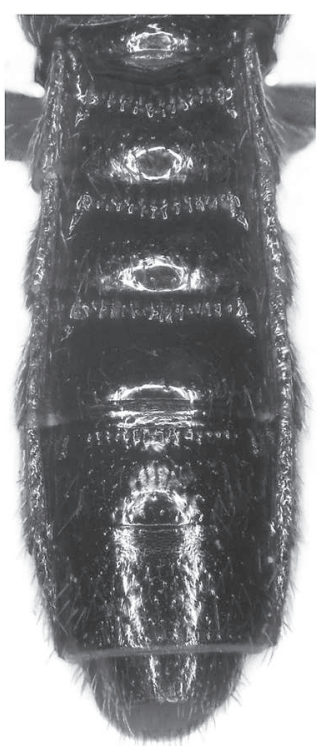

42

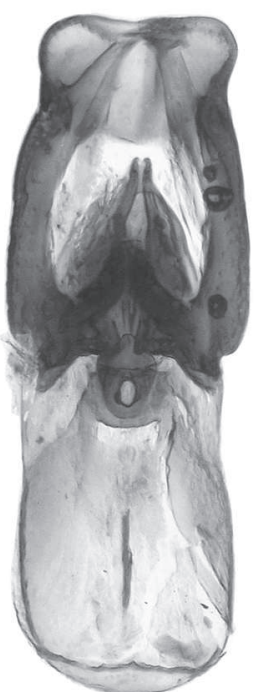

52
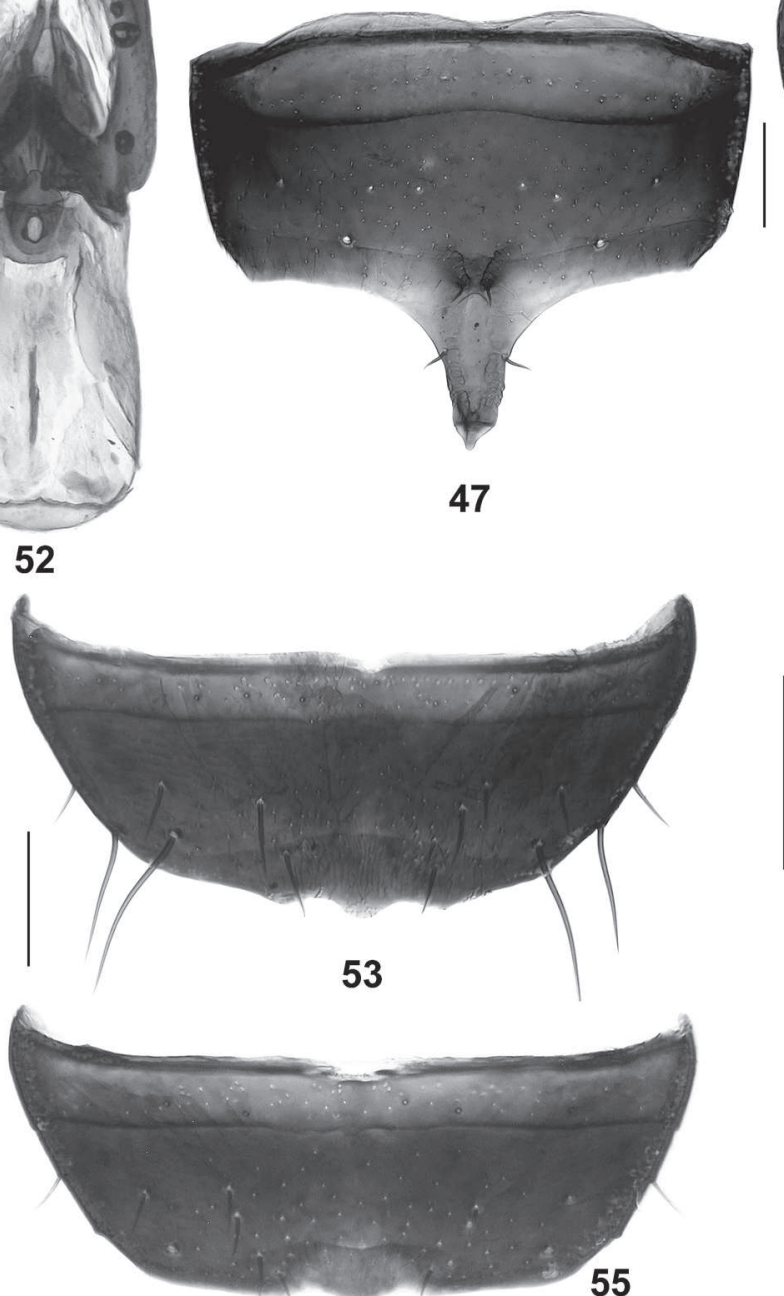
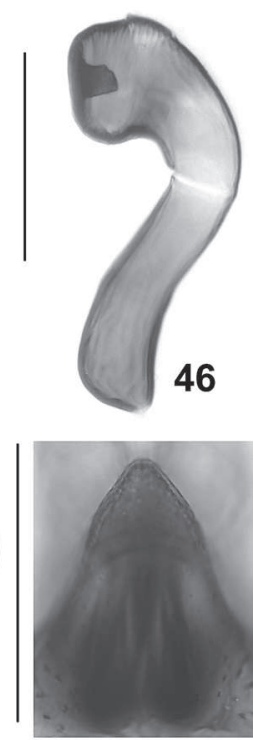

45

44

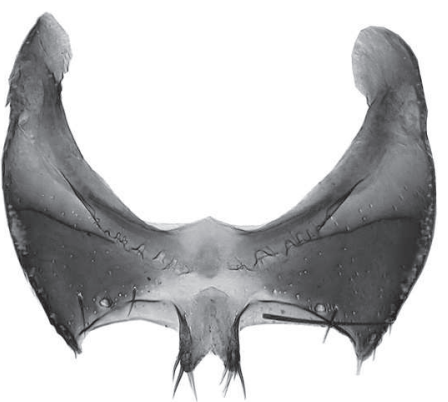

49

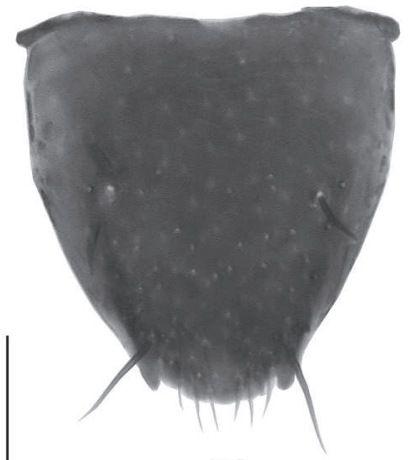

54

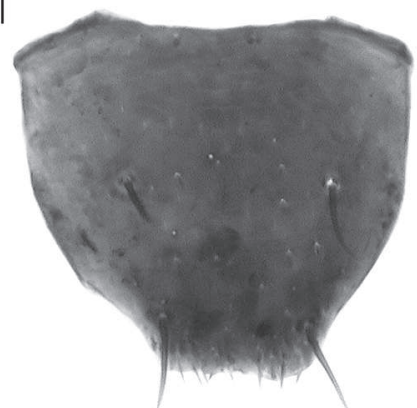

56

Figs 41-56: Tachyusa unguis (41-46), Anotylus hamatoides (47-52), Platystethus cephalotes (53-54), and P. laevis (55-56): forebody (41); abdomen (42); (median lobe of) aedeagus in lateral and in ventral view (43-44,51-52); apex of ventral process of aedeagus in ventral view (45); spermatheca (46); male sternite VII (47); posterior process of male sternite VII (48); male sternite VIII $(49,53,55)$; posterior process of male sternite VIII $(50)$; male tergite X $(54,56)$. Scale bars: 41-42: $0.5 \mathrm{~mm}$; $43-56: 0.1 \mathrm{~mm}$. 


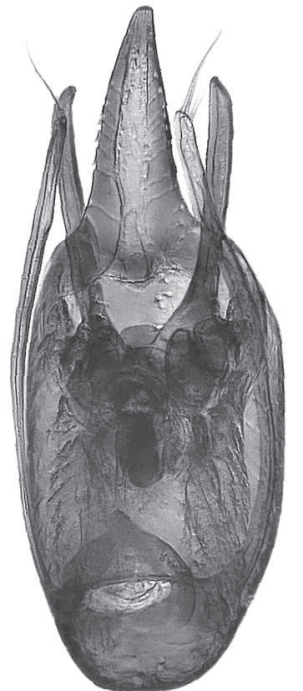

57

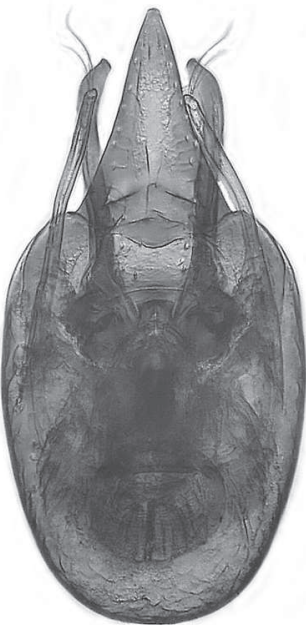

58

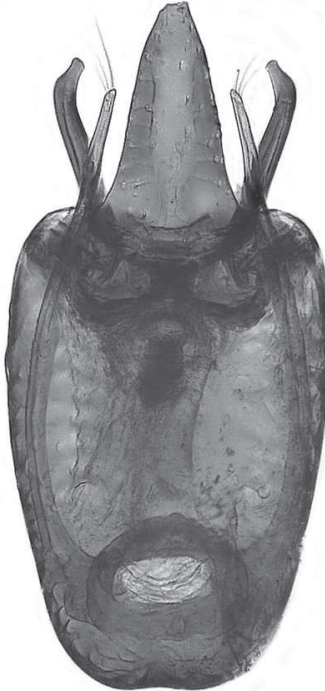

59

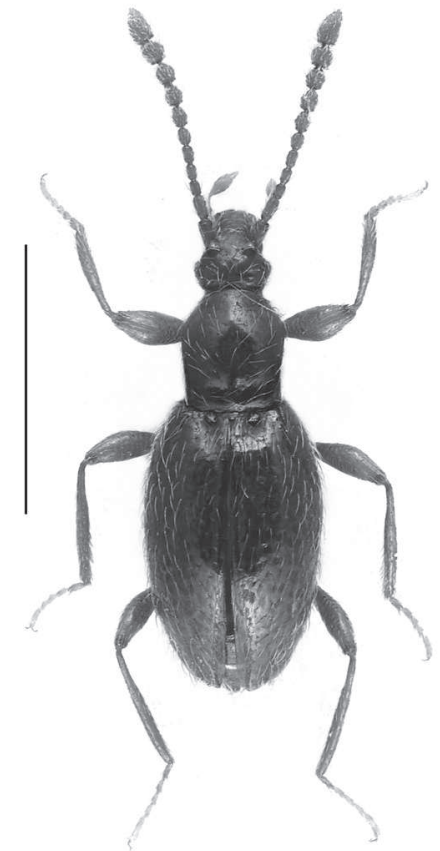

60

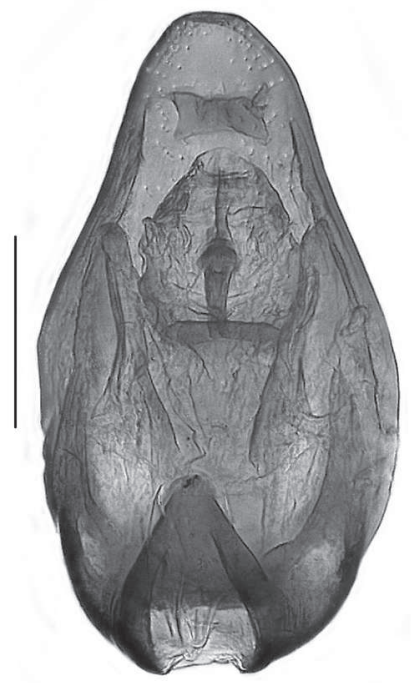

62

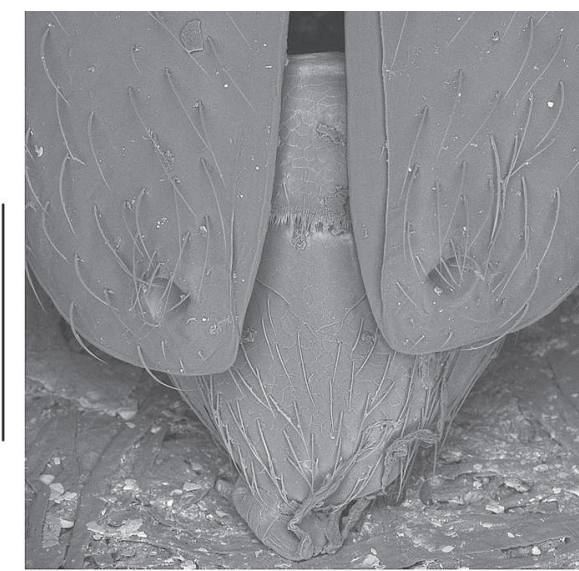

61

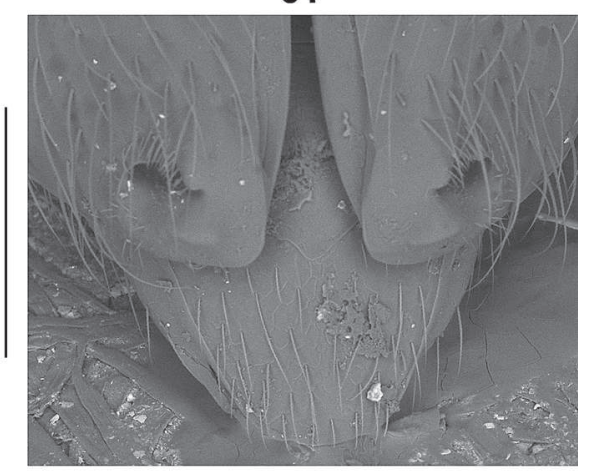

64

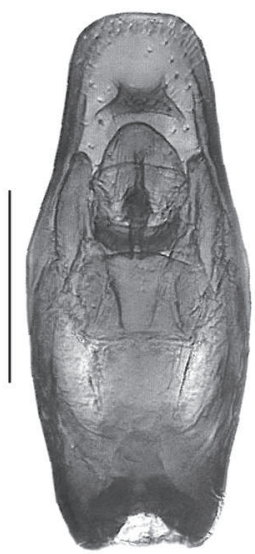

65

Figs 57-65: Euconnus longilaminatus (57), E. tavushus (58), E. karabakhus (59), Neuraphes gomarantsus (60-62), and N. syunikus (63-65): aedeagus in ventral view $(57-59,62,65)$; habitus $(60,63)$; posterior portion of elytra $(61,64)$. Scale bars: $60,63: 1.0 \mathrm{~mm}$; 61, 64: $0.2 \mathrm{~mm}$; 57-59, 62, 65: $0.1 \mathrm{~mm}$.

\subsection{Taxonomy}

\subsubsection{Omaliinae (by AdRIANo ZANETTI)}

Omalium kociani ZanetTI spec. nov.

urn:Isid:zoobank.org:act:5F24413E-6AB4-4D1C-B567-AB93C38380C9

(Figs 3, 7-10)

Type material: Holotype $\sigma^{*}$ : "ARMENIA, above Jermuk sifting of plant leavings near snow residues, $2400 \mathrm{~m}$ 39,839053N 45,693496E 21.V.2015 M. Kocian lgt. /
Omalium kociani n. sp. det. A. Zanetti 2016 / Holotypus" (MCSNV). Paratypes: $5 \sigma^{\star} \sigma^{\star}, 3$ 우 우: same data as holotype (cKoc, cZan, cAss). 
Etymology: This species is dedicated to the collector, Matús Kocian (Praha), specialist of Tachyporinae.

Description: Measurements $(n=9)$ : body length (with extended abdomen) $2.4-3.1 \mathrm{~mm}$; length of forebody from anterior margin of clypeus to posterior margin of elytra $1.53-1.88 \mathrm{~mm}$; head width $0.46-0.51 \mathrm{~mm}$; head length from clypeus to neck $0.35-0.42 \mathrm{~mm}$; width of pronotum $0.61-0.70 \mathrm{~mm}$; length of pronotum $0.42-$ $0.49 \mathrm{~mm}$; length of elytra from humeral angles to apex $0.70-0.83 \mathrm{~mm}$; length of elytral suture $0.61-0.72 \mathrm{~mm}$; width of elytra $0.75-0.90 \mathrm{~mm}$. Habitus as in Fig. 3. Coloration: head usually blackish, sometimes brown; pronotum reddish, sometimes with more or less extensive brownish discal area: elytra yellowish red; abdomen brownish with yellowish-brown paratergites; antennae, legs and mouth parts entirely yellowish.

Head moderately transverse, $1.2-1.3$ times as broad as long; temporal angles rounded but marked; two ridges extending from above insertion of antennae to compound eyes; surface without microsculpture, with dense and regular punctation forming some irregular striae between eyes and ante-ocellar pits, punctation anteriorly extending to the anterior margin of clypeus; sub-antennal impression rather wide, tentorial pits in front of ocelli long and linear, posteriorly extending to the rather small ocelli, the latter separated by a distance equal to that between ocellus and eye; space between tentorial pits and eyes elevated; neck clearly separated from the rest of the head, with dense punctation similar to that of head. Eyes small, slightly shorter than temples, without infra-orbital carina. Antenna rather thin and elongate, antennomere I elongate, twice as long as wide, II elongate ovoid, III-VIII longer than wide, gradually incrassate, IX-X subquadrate, XI longer than wide.

Pronotum transverse, on average 1.4 times as broad as long and 1.4 times as broad as head, widest in anterior half; anterior margin wider than posterior margin, anterior and posterior angles marked and slightly obtuse; lateral margins with trace of crenulation, weakly sinuate posteriorly; surface of pronotum irregular with three longitudinal elevations and two pits near lateral margin; punctation dense, similar to that of head, ground surface smooth without microsculpture.

Elytra small, 1.5 times as long (measured from humeral angles) and 1.3 times as broad as pronotum; punctation irregular and dense, coarser than on pronotum, interstices without microsculpture. Legs simple, with some spines in the apical portions of the meso- and metatibiae.

Abdomen with isodiametric microsculpture, decumbent pubescence and small wing-folding patches.

${ }^{*}$ : aedeagus as in Figs 7-8, median lobe with narrow base, strongly enlarged subapically; apex weakly curved dorsad in lateral view and without ventral elevations.

\%: accessory sclerites as in Fig. 9; spermatheca as in Fig. 10.
Comparative notes: The new species belongs to the Omalium caesum group as defined by ZaNeTti (1987): ventral process of the aedeagus proximally much narrower than the basal bulb, parameres distinctly dilated and of intricate structure distally, with very long apical setae, tentorial impressions line-shaped. According to ZANETTI (2002), the following representatives of the O. caesum group are distributed in Turkey, Cyprus and the Caucasus region: O. caesum Gravenhorst, 1806, O. littorale Kraatz, 1857, O. wunderlei Zanetti, 2002, and O. rugatum Mulsant \& ReY, 1880). They are all clearly distinguished from $O$. kociani by long elytra, rather uniform and dark colour, and by the shape of the median lobe of the aedeagus (ventral process not narrowed at the base). Omalium turcicum Smetana, 1867 is very similar in coloration and in general shape, but differs by wider elytra, smaller eyes, more rounded temples and confluent punctation of the head, and an impunctate clypeus. However, the only reliable distinguishing character is the shape of the aedeagus: in O. turcicum, the ventral process is gradually narrowed from the base to the obtuse apex (ZANETTI 2002: figure 58), whereas in O. kociani the ventral process is narrow at the base and enlarged in the middle, and the apex is more acute (Fig. 8). In populations of $O$. caesum with short elytra from the Alps, the ventral process of the aedeagus has a broad base (ZANETTI 2002: figure 55). Omalium cribriceps Fauvel, 1900, which is distributed in Iran, Iraq, Syria, and Turkey (SCHÜLKE \& SMETANa 2015), is very similar to O. kociani in coloration, habitus, and the punctation of the head, but has an aedeagus of completely different shape (ZANETTI 2002: figure 48) with wide median lobe and simple, linear parameres.

Distribution and natural history: This species is known only from the type locality in Armenia. The typical series was collected in an alpine meadow by sifting humus and rotting herbs near a small residual snow field.

\section{Dialycera minuta LUZE, 1906}

Phyllodrepa armena IAblokoff-Khnzorian, 1959: 66; syn. nov.

Type material examined: Dialycera minuta: Holotype o ${ }^{\top}$ : "Kana Gal. / J. Sahlb / 2177 / Spec.typ. / minuta m. det. Luze / Mus. Zool. H:fors spec. typ. No 1093 Dialycera minuta Luze." (MZH).

Phyllodrepa armena: Lectotype + , present designation: "Dzhrvezh Erevan ASSR 21.5.52" (cKhn).

Comment: This species will be treated in more detail in a revision of Dialycera currently in preparation. IABLOKOFF-KHNZORIAN (1959) reported minor and irrelevant differences between $D$. minuta and D. armena in body size and elytral punctation. Regarding the shape of the highly distinctive aedeagus, however, both are identical. In the original description of D. armena four 
specimens from the type locality and with the same date (21.V.1952) are mentioned. The sole syntype in the Khnzorian collection is designated as the lectotype. There are four additional specimens, including males, in $\mathrm{cKhn}$. They, too, were collected in the type locality, but on different dates (3.5.1951, 7.5.52, and 30.5.52) not mentioned in the original description.

\subsubsection{Proteininae (by VoLKER Assing)}

\section{Proteinus baculatus Assing spec. nov. urn:Isid:zoobank.org:act:3DDB2D69-A000-4FFA-AAC7-48CE4BAB7020 (Figs 11-14)}

Type material: Holotype ơ: "ARMENIA [27] - WSW Kapan, W Kajaran, 39 $09^{\prime} 22^{\prime \prime N}, 46^{\circ} 06^{\prime} 13^{\prime \prime E}, 2050 \mathrm{~m}$, mixed forest, 10.VII.2018, V. Assing / Holotypus ơ Proteinus baculatus sp. n. det. V. Assing 2018" (cAss). Paratypes: $10^{\text {: }}$ "ARMENIA [AR16-08] N Yerevan, NW Hrazdan, $40^{\circ} 33^{\prime} 45^{\prime \prime} \mathrm{N}, 44^{\circ} 23^{\prime} 41^{\prime \prime} \mathrm{E}, 2000 \mathrm{~m}$, mixed deciduous forest margin, litter sifted, 28.VI.2016, leg. M. Schülke" (MNB); $1 \mathrm{o}^{\text {: }}$ "TR. - Erzurum, $40 \mathrm{~km}$ NW Tortum, 2100 m, Mescit Dağları, pine forest, ca. $40^{\circ} 36 \mathrm{~N}, 41^{\circ} 23 \mathrm{E}, 20 . \mathrm{VI} .1998$, Solodovnikov" (cAss).

Etymology: The specific epithet is an adjective derived from the Latin noun baculus (stick, rod) and alludes to the rod-shaped sclerotized structure in the internal sac of the aedeagus.

Description: Body length 2.1-2.4 mm; length of forebody 1.4-1.6 mm. Habitus as in Fig. 11. Coloration: body black; legs dark-yellowish; antennae black with antennomere I pale-reddish.

Head and pronotum with pronounced isodiametric microsculpture and with extremely fine, barely noticeable sparse punctation. Pronotum approximately 1.8 times as broad as long and 1.4 times as broad as head, posterior margin very finely margined (visible only at a magnification of $100 \mathrm{x}$ ). Elytra approximately 1.9 times as long as pronotum; interstices without microsculpture. Process of mesoventrite with a moderately pronounced median keel (Fig. 12).

$0^{7}$ : protarsomere I dilated and very long, slightly longer than the combined length of protarsomeres II-V; mesotibia smoothly curved, with few peg setae and somewhat flattened in apical fourth; metatibia unmodified; aedeagus (Figs 13-14) $0.36-0.38 \mathrm{~mm}$ long; ventral process apically very acute both in lateral and in ventral view; internal sac with a long and broad black membranous tube and with a rod-shaped sclerotized structure best visible in ventral view.

Comparative notes: Based on the elongated male protarsomere I, P. baculatus belongs to the $P$. crenulatus group, which previously included five species in the West Palaearctic region (Assing 2007a). It is distinguished from P. crenulatus and other species of this group by a reddish antennomere I and by the male sexual characters, particularly by the shapes of the ventral process and of the internal structures of the aedeagus. For illustrations of the species of the P. crenulatus group see Assing (2007a).

Two Proteinus species were described from Ordubad in Azerbaijan, close to the border with Armenia: P. planicollis Reitter, 1905 and P. reflexicollis Reitter, 1905 (type material of both species examined). The new species is distinguished from them by completely black coloration of the body, by a more convex (cross-section) pronotum, and additionally as follows:

from P. planicollis by smaller size (P. planicollis: length of forebody 1.7-1.8 $\mathrm{mm}$ ), a less broad habitus, a less transverse pronotum with indistinctly bordered lateral margins (P. planicollis: pronotum 1.96-2.00 times as broad as long and 1.53 times as broad as head), shorter elytra (P. planicollis: elytra 2.0 times as long as pronotum), an elongate male protarsomere I (unmodified in P. planicollis), and an aedeagus of different shape;

from P. reflexicollis by indistinctly bordered lateral margins of the pronotum.

The aedeagi of the two male type specimens of $P$. reflexicollis are either missing or deformed and completely bleached.

Distribution and natural history: This species is currently known from two localities in North and South Armenia and from one locality in Northeast Anatolia. The Armenian specimens were sifted from leaf litter and grass roots in mixed deciduous forests. The altitudes range from 2000 to $2100 \mathrm{~m}$. The male from Turkey is slightly teneral.

\subsubsection{Pselaphinae (by Volker BRACHAT)}

\section{Bryaxis armeniacus BRACHAT spec. nov.} urn:Isid:zoobank.org:act:01F595CB-9F3B-4DEE-8A4D-C97F39DE26E1 (Figs 15, 17)

Type material: Holotype ơ': "ARMENIA [4] - N Yerevan, NW Hrazdan, 404 $41^{\prime} 40^{\prime} \mathrm{N}, 44^{\circ} 29^{\prime} 16^{\prime} \mathrm{E}, 2500 \mathrm{~m}$, W-slope, sifted, 26.VI.2016, V. Assing / Bryaxis armeniacus spec. nov. $0^{7}$, det. Brachat 10.2018 / Holotypus" (cBra). Paratypes: $40^{\top} \sigma^{\top}, 3$ 우 $\circ$ : same data as holotype (cBra); 6 o $^{\top} 0^{7}, 6$ 우 9 : same data, but leg. Schülke (cSch).

Etymology: The specific epithet is an adjective derived from Armenia, where the species may be endemic.

Description: Body length $1.6-1.8 \mathrm{~mm}$. Coloration: reddish-brown with the antennae and the maxillary palpi slightly paler. Pubescence on average rather long and nearly depressed; head with scattered long and erect setae. 
Head weakly transverse, $0.32-0.34 \mathrm{~mm}$ long and $0.34-$ $0.36 \mathrm{~mm}$ broad, more or less distinctly punctate in anterior half; frontal lobe $0.18 \mathrm{~mm}$ broad; vertex with unmodified median keel; postocular region weakly convex in dorsal view. Antennomeres III-XI $0.50 \mathrm{~mm}$ long; antennomeres III distinctly oblong, IV-VIII globulous and approximately as long as broad, IX-XI forming a distinct club, X more transverse than IX, and XI longer than combined length of VIII-X. Maxillary palpi rather short; palpomeres II and III with numerous tubercles, especially ventrally; palpomere IV weakly petiolate, $0.13 \mathrm{~mm}$ long and $0.05 \mathrm{~mm}$ broad.

Pronotum $0.36-0.38 \mathrm{~mm}$ long and $0.40-0.42 \mathrm{~mm}$ broad, convex in cross-section, broadest in anterior third, glabrous, punctate only near posterior margin.

Elytra $0.60-0.64 \mathrm{~mm}$ long and $0.68-0.72 \mathrm{~mm}$ broad, slightly more than one-tenth as broad as long; humeral angles marked; punctation distinct, partly coarse.

$\sigma^{*}$ : head ventrally with deep semicircular gular impression, anterior margin of this impression elevated; eyes small, each composed of 12-15 ommatidia; antenna: scapus $0.145 \mathrm{~mm}$ long and $0.110 \mathrm{~mm}$ broad, dorsally slightly dilated, inner margin with a small tubercle (Fig. 17); pedicel (Fig. 17) oval, $0.08 \mathrm{~mm}$ long and $0.06 \mathrm{~mm}$ broad in dorsal view; inner margin weakly, outer margin distinctly convex; metaventrite weakly convex in cross-section, posteriorly flat and with triangular impression; legs robust; pro- and metafemora dilated; profemur with depression near base; metatrochanter posteriorly with keel-shaped dilatation; protibia slightly dilated and with deep emargination in apical third; metatibia distinctly dilated, in apical half with projecting tooth, a deep emargination, and a small spine at apical margin; modifications of male legs are subject to intraspecific variation and may be more or less distinctly reduced; aedeagus $0.40-0.43 \mathrm{~mm}$ long and shaped as in Fig. 15.

: : eyes small, composed of 8-12 ommatidia; antenna with subcylindrical scapus (length: $0.14 \mathrm{~mm}$; width: $0.07 \mathrm{~mm}$ ) and with oval pedicel (length $0.07 \mathrm{~mm}$; width $0.06 \mathrm{~mm}$ ); metaventrite flattened posteriorly.

Comparative notes: Bryaxis armeniacus belongs to the B. clavipes group (see Besuchet \& Kurbatov 2007). It is similar to B. nivarius Besuchet \& Kurbatov, 2007 (Northeast Turkey), but distinguished by smaller size and the internal structures of the aedeagus.

Distribution and natural history: This species is currently known only from one locality to the northwest of Hrazdan, North Armenia. The specimens were sifted from litter and grass roots in a grassy west slope with scattered Salix at an altitude of $2500 \mathrm{~m}$. For a photo of the type locality see figure 41 in Assing (2016b).

\section{Bryaxis meghruicus BRACHAT spec. nov. urn:Isid:zoobank.org:act:AA6E7E2A-4450-48E8-8048-8061C3C6DF5C (Figs 16, 18)}

Type material: Holotype ơ [left antennomeres X and XI and right hind leg missing]: "ARMENIA [29] - ca. $30 \mathrm{~km}$

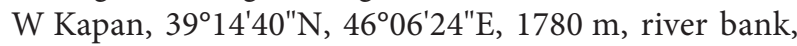
11.VII.2018, V. Assing / Bryaxis meghruicus spec. nov. $\sigma^{*}$, det. Brachat 10.2018 / Holotypus" (cBra).

Etymology: The specific epithet is an adjective derived from Meghru, the name of the mountain range where the type locality is situated.

Description: Body length $1.6 \mathrm{~mm}$. Coloration: dark reddish-brown with the antennae, the maxillary palpi, and the legs slightly paler. Pubescence rather long and sub-erect; head with scattered long and erect setae.

Head distinctly transverse, $0.26 \mathrm{~mm}$ long and $0.34 \mathrm{~mm}$ broad, weakly punctate in anterior half; frontal lobe $0.17 \mathrm{~mm}$ broad; vertex with unmodified median keel. Eyes large and distinctly projecting, composed of approximately 25 ommatidia, slightly longer than the weakly convex postocular region in dorsal view. Antenna rather short; antennomeres III-XI $0.46 \mathrm{~mm}$ long; antennomeres III weakly oblong, IV-VII globulous and approximately as long as broad, VIII weakly transverse, IX-XI forming a distinct club, IX and X distinctly transverse, and XI slightly broader than $\mathrm{X}$ and longer than combined length of VII-X. Maxillary palpi rather short; palpomeres II and III with few tubercles; palpomere IV petiolate, $0.22 \mathrm{~mm}$ long and $0.09 \mathrm{~mm}$ broad.

Pronotum $0.36 \mathrm{~mm}$ long and $0.38 \mathrm{~mm}$ broad, convex in cross-section, broadest in anterior third.

Elytra $0.60 \mathrm{~mm}$ long and $0.64 \mathrm{~mm}$ broad, with fine and posteriorly more distinct punctation; humeral angles marked.

$\sigma^{\star}$ : head ventrally with short transverse gular impression, anterior margin of this impression elevated; antenna: scapus $0.15 \mathrm{~mm}$ long and $0.12 \mathrm{~mm}$ broad, dorsally slightly dilated, inner margin with a small flat tubercle (Fig. 18); pedicel (Fig. 18) oval and unmodified, $0.060 \mathrm{~mm}$ long and $0.055 \mathrm{~mm}$ broad in dorsal view; metaventrite with median sulcus posteriorly; legs robust; pro- and metafemora dilated; profemur with depression near base; protibia dilated and with deep emargination in apical third; metatibia distinctly dilated, in apical half with projecting tooth, a deep emargination, and a pronounced spine at apical margin; aedeagus $0.41 \mathrm{~mm}$ long and shaped as in Fig. 16.

Comparative notes: Bryaxis meghruicus belongs to the B. clavipes group. It is distinguished from other species of this group by the internal structures of the aedeagus and by the combination of other characters. 
Distribution and natural history: The type locality is situated some $30 \mathrm{~km}$ to the west of Kapan, South Armenia. The holotype was floated from gravel on a river bank at an altitude of $1780 \mathrm{~m}$.

\subsubsection{Tachyporinae (by MıCHAEL SCHÜLKE)}

\section{Mycetoporus silvaticus IABLOKOFF-KHnZORIAN, 1962} (Fig. 4)

Mycetoporus dispersus SchüLKe \& KocIAN, 2000, syn. nov.

Type material: Holotype $0^{\star}$ : "Mravyan 18.6.60 Khnz. / Mycetoporus silvaticus Khn. Khnzorian det. / Typus / Holotype / Mycetoporus silvaticus Iablokoff-Khnzorian det. M. Schülke 2018” (IZAY).

Comment: When the species of the Mycetoporus nigricollis group were revised (Schülke \& Kocian 2000), no type material of Mycetoporus silvaticus was available. The species was described based on four specimens collected in different localities in northern and southern Armenia (Zaghkadsor, Mravyan, Vachagan). In the original description, the author compared this species with M. corpulentus LuzE, 1902, a species which was unknown from the Caucasus region at that time.

An examination of the male holotype from Mravyan (Fig. 4) revealed that the large internal structures of the aedeagus are identical to those of $M$. dispersus. As $M$. silvaticus is the senior name, it takes priority, rendering $M$. dispersus a junior synonym.

\subsubsection{Aleocharinae (by Volker Assing)}

$$
\text { Aleochara (Ceranota) subtumida (HосннuтH, 1849) }
$$

Aleochara (Polychara) khnzoriani Amiryan, 1999b: 62 f.; syn. nov.

Type material examined: Holotype + : "Armenia, Goris, sel. [village] Tatev, pod kamnem [under stones], 21.05.88, c. Kalashian M. / Type / Aleochara khnzoriani sp. n. Det. Amiryan A / Aleochara subtumida (Hochhuth), det. V. Assing 2018" (IZAY).

Comment: In the original description of Aleochara khnzoriani, which is based on a unique female, AMIRYAN (1999b) attributed the species to the subgenus Polychara Mulsant \& Rey, 1874, now a synonym of Xenochara Mulsant \& Rey, 1874. An examination of the holotype, however, revealed that the specimen belongs to the subgenus Ceranota Stephens, 1839 and is conspecific with A. subtumida. Hence the synonymy proposed above.

\section{Aleochara (Xenochara) fugax IABLOKOFF-KHNZORIAN,} 1962

(Figs 19-21)

Aleochara fugax IABLoKoff-KHnZorian, 1962: $112 \mathrm{f}$.

Material examined: 1 9 : "Erevan, Dzhrvezh, ASSR. 24.5.48" (cKhn).

Comment: Aleochara fugax, whose original description is based on a holotype and one paratype from Armenia (Vedi, Vinogradov sandstone cave, 8.IV.1958), is listed as Aleochara incertae sedis in SchüLKe \& SMetana (2015). An examination of the above specimen from the Khnzorian collection, evidently not the holotype, revealed that A. fugax is highly similar and undoubtedly closely related to A.parvicornis Fauvel, 1900 of the subgenus Xenochara. It very much resembles A. parvicornis in habitus, antennal morphology, coloration, dense punctation, and the derived chaetotaxy of tergite VIII, but is distinguished by less dense punctation of the whole body (particularly so on the abdominal tergites VI and VII) and by a more slender distal portion of the spermathecal capsule. Habitus, posterior margin of tergite VIII, and spermatheca of A. fugax are illustrated in Figs 19-21. For illustrations of A. parvicornis see Assing (2009a).

The Armenian record of A. parvicornis by Likovský (1971) most likely refers to A. fugax.

\section{Atheta (Paralpinia) meghruica Assing spec. nov. urn:Isid:zoobank.org:act:4673F1AA-F8C1-4524-AFA3-99E4E7F502FF (Figs 22-28)}

Type material: Holotype o : "ARMENIA [35] - 25 km SW Kapan, $39^{\circ} 04^{\prime} 01^{\prime \prime} \mathrm{N}, 46^{\circ} 16^{\prime} 10^{\prime \prime} \mathrm{E}, 2150 \mathrm{~m}$, near stream, sifted, 14.VII.2018, V. Assing / Holotypus o Atheta meghruica sp. n. det. V. Assing 2018” (cAss). Paratypes: $1 o^{*}$ : same data, but leg. Schülke (cSch); 1 i : "ARMENIA [32] - SW Kapan, Mt. Khustup, 3907'50"N, 46¹9'55"E, 2980 m, litter near spring, 12.VII.2018, V. Assing” (cAss).

Etymology: The specific epithet is an adjective derived from Meghru, the name of the mountain range where this species was discovered and where it may be endemic.

Description: Body length $2.2-2.6 \mathrm{~mm}$; length of forebody $1.1 \mathrm{~mm}$. Habitus as in Fig. 22. Coloration: body black; legs blackish-brown to blackish, with paler tarsi; antennae black.

Head (Fig. 23) weakly transverse, weakly dilated posteriad; punctation rather sparse and extremely fine; interstices with pronounced microsculpture composed of isodiametric meshes. Eyes distinctly convex, protruding from lateral contours of head, slightly shorter than distance from posterior margin of eye to posterior carina of head in dorsal view. Antenna $0.65 \mathrm{~mm}$ long and shaped as in Fig. 24. 
Pronotum (Fig. 23) approximately 1.15 times as broad as long and 1.15 times as broad as head, broadest in anterior half; pubescence of midline directed anteriad; punctation and microsculpture similar to those of head.

Elytra (Fig. 23) approximately as long as pronotum; microsculpture pronounced, composed of isodiametric meshes, these meshes larger than those on head and pronotum; punctation extremely fine, barely noticeable even at high magnification $(100 \mathrm{x})$. Hind wings fully developed.

Abdomen (Fig. 25) slender, distinctly narrower than elytra; punctation fine, moderately dense on tergite III, decreasing in density from tergite III to tergite VII; interstices with distinct microsculpture composed of a mix of large isodiametric and short transverse meshes, on tergites VII-VIII predominantly of isodiametric meshes; posterior margin of tergite VII with palisade fringe; posterior margin of tergite VIII without evident sexual dimorphism, posterior margin with shallow concavity in the middle.

$\mathrm{o}^{*}$ : sternite VIII with strongly convex posterior margin; median lobe of aedeagus (Figs 26-27) $0.23 \mathrm{~mm}$ long, ventral process of broadly triangular shape in ventral view; crista apicalis strongly projecting, but with very narrow membranous portion.

ㅇ: posterior margin of sternite VIII smoothly convex, with long and moderately modified marginal setae; spermatheca shaped as in Fig. 28.

Comparative notes: Atheta meghruica is distinguished from A. schneideri (Eppelsheim, 1889), the only other representative of the subgenus Paralpinia BenICK, 1974 known from the Caucasus region and Armenia, by even smaller size, a more slender body, shorter and much finer antennae with more transverse antennomeres $\mathrm{V}-\mathrm{X}$, a less convex pronotum (cross-section), a much smaller median lobe of the aedeagus (A. schneideri: length of median lobe nearly $0.5 \mathrm{~mm}$ ), and a spermatheca with a shorter and less slender distal portion and with a shorter proximal portion.

Distribution and natural history: The high altitudes of the localities (2190-2980 m) and the absence of records from other regions suggest that Atheta meghruica is probably regionally endemic in the Meghru mountain range in South Armenia. The male specimens were sifted from litter and debris near a stream, the female from roots and debris near a spring. Both males are slightly teneral.

Bellatheta khustupica Assing spec. nov. urn:Isid:zoobank.org:act:C342B3DE-9EE5-4950-85BD-817DBEE6FE3E (Figs 1-2, 5, 29-35)

Type material: Holotype $o^{*}$ : "ARMENIA [31] - SW Kapan, Mt. Khustup, 3907'52"N, 46¹9'43"E, 3060 m, soil near rocks, 12.VII.2018, V. Assing / Holotypus o Bellatheta khustupica sp. n. det. V. Assing 2018” (cAss). Paratypes: $10^{\text {त }}, 4$ 우 우: same data as holotype (cAss).

Etymology: The specific epithet is an adjective derived from Khustup, the name of the mountain where this species is probably endemic.

Description: Body length 1.9-2.4 mm; length of forebody $0.8-1.0 \mathrm{~mm}$. Habitus as in Fig. 5. Coloration: forebody pale-brown to brown; abdomen blackish-brown with the apex (segments VIII-X and posterior margin of segment VII) reddish-brown; legs yellow; antennae darkyellow to pale-brown with antennomeres I-II yellow.

Head (Fig. 29) weakly transverse, somewhat dilated posteriad; punctation extremely fine, barely visible even at high magnification $(100 \mathrm{x})$, and sparse; interstices with shallow microreticulation and glossy. Eyes very small, composed of approximately 6-8 ommatidia with pigmentation. Antenna short, approximately $0.5 \mathrm{~mm}$ long, and distinctly incrassate apically; antennomeres III transverse, IV-X strongly transverse, approximately three times as broad as long, and gradually increasing in width. Maxillary palpi short; palpomere III weakly oblong. Pronotum (Fig. 29) approximately 1.15 times as broad as long and 1.10-1.14 times as broad as head, broadest near anterior angles, and distinctly tapering posteriad; punctation similar to that of head; microsculpture slightly more distinct than that of head.

Elytra (Fig. 29) very short, approximately 0.6 times as long as pronotum; punctation similar to that of pronotum; interstices with distinct microreticulation. Hind wings completely reduced.

Abdomen (Fig. 30) broader than elytra, broadest at segment VII; punctation fine and sparse; interstices with distinct, but shallow microreticulation, glossy; posterior margin of tergite VII without palisade fringe; posterior margin of tergite VIII broadly convex.

$\mathrm{o}^{\star}$ : sternite VIII much longer than tergite VIII; median lobe of aedeagus (Figs 31-33) 0.28-0.30 mm long, strongly curved in lateral view; crista apicalis very long and without distinct membranous portion.

\% : sternite VIII only indistinctly longer than tergite VIII; spermatheca shaped as in Figs 34-35.

Comparative notes: The genus Bellatheta RoubAL, 1928 is discontinuously distributed across the Palaearctic region. In the West Palaearctic region exclusive of Middle Asia, it is represented by nine species. Except for the winged and moderately widespread B. fatrica RouBAL, 1928 (distributed in the Balkans, Italy, and Central Europe) and B.palata (BENICK, 1970) (recorded from Bosnia-Herzegovina, Bulgaria, Greece, and Armenia), the species are micropterous and locally endemic in mountain ranges of Spain (B. aragonica (Assing, 2001)), Italy (B. kappi (Assing, 2002); B. rosai (PACE, 1978)), Lebanon (B. besucheti (PACE, 1982)), mainland Greece (B. renominata (Likovskŕ, 1984)), and Crete (B. albimontis Assing, 2015; B. idana Assing, 2015). Bellatheta khustupica is 
distinguished from all of them by the primary sexual characters. It is readily separated from B. palata, the only other species of the genus recorded from Armenia, by much smaller eyes, smaller body size, paler coloration, much shorter elytra, completely reduced hind wings, and the absence of a palisade fringe at the posterior margin of tergite VII alone.

Distribution and natural history: Bellatheta khustupica is probably locally endemic to the alpine regions of Mount Khustup in South Armenia and most likely the only truly locally endemic species of Staphylinidae in Armenia. The specimens were sifted from soil and the roots of Rumex alpinus at the base of large rocks on a north slope at an altitude of $3060 \mathrm{~m}$. The type locality and the habitat where the type specimens were collected are illustrated in Figs 1-2.

\section{Calodera alticola Assing spec. nov.}

urn:Isid:zoobank.org:act:3BF46F7B-FBDB-4F1B-B0D0-89D669B021C2

(Figs 6, 36-40)

Type material: Holotype $\sigma^{\top}$ : "ARMENIA [37] - $30 \mathrm{~km}$

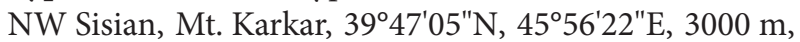
wetland, sifted, 15.VII.2018, V. Assing / Holotypus o Calodera alticola sp. n. det. V. Assing 2018” (cAss).

Etymology: The specific epithet is a noun in apposition meaning inhabitant of high altitude. It alludes to the high elevation of the type locality.

Description: Body length $2.7 \mathrm{~mm}$; length of forebody $1.25 \mathrm{~mm}$. Habitus as in Fig. 6. Coloration: body black; legs blackish-brown with paler brown protibiae and tarsi; antennae blackish-brown with antennomeres I-II brown. Head (Fig. 36) approximately as long as broad, of suborbicular shape; posteriorly without neck, with broad and short constriction; punctation extremely fine, barely visible even at high magnification $(100 \mathrm{x})$, and moderately dense; interstices with distinct microreticulation. Eyes rather large and weakly convex, nearly as long as postocular region in dorsal view. Antenna $0.75 \mathrm{~mm}$ long; antennomeres III weakly oblong, IV-X approximately twice as broad as long and gradually increasing in width.

Pronotum (Fig. 36) weakly transverse, approximately 1.05 times as broad as long and approximately 1.15 times as broad as head; punctation and microsculpture similar to those of head.

Elytra (Fig. 36) slightly longer than pronotum; punctation extremely fine and dense; microsculpture distinct, composed of relatively large meshes. Hind wings fully developed.

Abdomen (Fig. 37) narrower than elytra, broadest at segments VI-VII; tergites III-V with deep, tergite VI with slightly shallower anterior impression; punctation extremely fine and dense; interstices with pronounced microreticulation; posterior margin of tergite VII with palisade fringe.

$0^{\top}$ : posterior margin of sternite VIII broadly convex; median lobe of aedeagus (Figs 38-40) $0.32 \mathrm{~mm}$ long; apical internal structures of distinctive shape in ventral view.

q: unknown.

Comparative notes: Calodera alticola is characterized particularly by the shape of the apical internal structures of the aedeagus in ventral view. In general appearance, C. alticola is similar to C. aethiops (GRAVENHORST, 1802). It additionally differs from this species by even smaller body size, a shorter and broader posterior constriction of the head, a weakly transverse and smaller pronotum (in relation to the head and the pronotum) with finer punctation, shorter elytra, and darker legs. For illustrations of C. aethiops and other small West Palaearctic species see Assing (1996).

Distribution and natural history: The type locality is situated in Mount Karkar to the northwest of Sisian, Armenia. The holotype was sifted from moist debris near the shore of a small lake at an altitude of $3000 \mathrm{~m}$.

\section{Tachyusa unguis Assing spec. nov. urn:Isid:zoobank.org:act:A4B4E5C2-EEB2-4146-B6F2-27D5873D5AEB (Figs 41-46)}

Type material: Holotype $0^{\star}$ : "S - ARMENIA: $25 \mathrm{~km}$ N Kapan, nr. Norarachadzor village, "Davidbeksoe" water res., 13.5.2001, ca. $1400 \mathrm{~m}$, leg. Shaverdo (71A) / Holotypus ơ Tachyusa unguis sp. n. det. V. Assing 2018" (NHMW). Paratypes: $30^{\top} \sigma^{\top}, 1$ 우 [without head]: same data as holotype (NHMW, cAss).

Etymology: The specific epithet is a noun in apposition meaning claw. It alludes to the large claw-shaped internal structures of the aedeagus.

Description: Body length $3.0-3.4 \mathrm{~mm}$; length of forebody $1.5-1.7 \mathrm{~mm}$. Coloration: head black; pronotum brown to blackish-brown, sometimes with weak bronze hue; elytra reddish-brown with the anterior and lateral portions slightly darker, with or without weak bronze hue; abdomen moderately bicoloured with segments III-V reddish to reddish-brown and the apical segments bicoloured; legs reddish-yellow; antennae dark-brown with the basal 2-3 antennomeres reddish.

Head (Fig. 41) weakly transverse, with very indistinct median impression at most; punctation fine and moderately dense; interstices with very shallow, partly obsolete microreticulation. Eyes nearly as long as distance from posterior margin of eye to posterior constriction of head in dorsal view. Antenna 1.1-1.2 mm long; antennomeres II and III slender and of subequal length, IV approximately 1.5 times as long as broad, $\mathrm{V}-\mathrm{X}$ of gradually 
decreasing length and decreasingly oblong, and $\mathrm{X}$ indistinctly oblong or approximately as long as broad. Pronotum (Fig. 41) indistinctly transverse and slightly broader than head; disc with or without very shallow and broad median impression or depression in posterior portion; lateral margins not sinuate, converging posteriad in straight line; punctation fine and moderately dense, usually slightly less fine than that of head; interstices without microsculpture.

Elytra (Fig. 41) slightly shorter than pronotum; punctation slightly finer and less dense than that of pronotum. Hind wing fully developed. Metatarsomere I longer than II, but shorter than the combined length of II and III.

Abdomen (Fig. 42) slightly narrower than elytra, broadest at segment VI, rather weakly constricted anteriorly; tergite IV (without paratergites) approximately twice as broad as long; tergites III-V with very deep anterior impressions, these impressions with coarse punctation partly separated by irregular longitudinal keels; tergite VI anteriorly with a narrow band of coarse punctation; remainder of tergal surfaces with rather sparse and fine, but distinct punctation; tergites III-V without, tergites VII-VIII with shallow microsculpture (at least posteriorly), tergite VI with or without very shallow traces of microsculpture; pubescence rather long and mostly suberect; posterior margin of tergite VII with palisade fringe; posterior margin of tergite VIII weakly concave.

$\sigma^{*}$ : posterior margin of sternite VIII convex; median lobe of aedeagus (Figs 43-45) approximately $0.4 \mathrm{~mm}$ long and of rather robust shape; ventral process short, in ventral view of broadly triangular shape; internal sac with a pair of large claw-shaped sclerotized structures.

\%: posterior margin of sternite VIII concave in the middle; spermatheca shaped as in Fig. 46.

Comparative notes: Based on the shape of the ventral process, on the presence of claw-shaped structures in the internal sac of the aedeagus, and on the characters indicated by PAśniK (2006) (punctation of abdomen; pronotum of trapezoid shape), T. unguis is assigned to the T. impressa group. It is distinguished from all the species of this group by the shape of the ventral process of the aedeagus and by the larger claw-shaped internal structures of the aedeagus. It differs from geographically close representatives of the T. impressa group as follows:

from T. impressa (Caucasus region) by a larger and more robust aedeagus with larger claw-shaped structures, with a longer crista apicalis not clearly separated from the aedeagal capsule, and with a shorter and basally much broader ventral process (ventral view), by a usually less distinctly impressed pronotum with finer punctation, and by slightly coarser punctation separated by more distinct carinae in the anterior impressions of tergites III-V; from T. nitella FaUvel, 1895 (widespread in the West Palaearctic region), with which it shares a broadly triangular ventral process (ventral view) and the shape of the crista apicalis of the aedeagus (lateral view), by paler coloration (especially of the legs, basal antennomeres, and anterior segments of the abdomen), sparser and finer punctation of the pronotum, and by a distinctly larger and more robust aedeagus with much larger claw-shaped internal structures (T. nitella: median lobe only approximately $0.3 \mathrm{~mm}$ long);

from T. loebli PAśNIK, 2006 (Turkey) by paler coloration (especially of the legs, basal antennomeres, elytra, and anterior segments of the abdomen), finer punctation of the pronotum, the absence of a distinct median impression on the pronotum, and by a more robust aedeagus with a much broader and shorter ventral process, with a crista apicalis of different shape, and by much larger clawshaped internal structures.

For illustrations of the primary sexual characters of other Tachyusa species see PAśniK (2006).

Comment: According to PAŚNIK (2006), T. turcica PAŚNIK, 2006 from Turkey belongs to the T. impressa group, too. However, based on personal observations, this name is most likely a junior synonym of $T$. impressa.

Distribution and natural history: The type locality is situated to the north of Kapan. The specimens were collected on the bank of Khashuni river at an altitude of about $1400 \mathrm{~m}$.

\subsubsection{Oxytelinae (by MıcHAEL SchÜLKE)}

\section{Anotylus hamatoides ScHüLKE spec. nov. urn:Isid:zoobank.org:act:0167E052-EBAB-421A-BB72-39E33C1EDC97 (Figs 47-52)}

Type material: Holotype $0^{\top}$ : "ARMENIA, Lori prov., Teghut mine, near artef. pond $41.0907^{\circ} 044.8117^{\circ}$ (soil traps) 990 m, 23.07.-23.08.2015 Karagyan \& Kalashian leg. / +6 / Holotypus- $0^{-}$Anotylus hamatoides spec. nov. det. M. Schülke 2018” (IZAY). Paratypes: 1 9 : same data as holotype but "+14" (IZAY); $10^{7}$ : "ARMENIA, Lori prov., Teghut mine, N41.0967 ${ }^{\circ}$, E44.8218 920 m, 22.08.01.10.2013, Kalashian leg. / (soil traps Ser.2)" (cSch); 1 \%: "ARMENIA, Lori prov., Teghut mine, near artef. pond $41.0907^{\circ} 044.8117^{\circ}$ (soil traps) $990 \mathrm{~m}, 23.08 .-23.09 .2015$ Karagyan \& Kalashian leg. / +2" (cSch); 1 \%: "ARMENIA, Lori prov., Teghut mine, N41.0743 ${ }^{\circ} \mathrm{E} 44.8399^{\circ}, 1105 \mathrm{~m}$, 22.08.-01.10.2013, Kalashian leg. / (soil traps Ser.3) / Anotylus fairmairei Pand." (IZAY); 1 \%: "ARMENIA, Lori prov., Teghut mine, N41.0910 $\mathrm{E} 44.8514^{\circ}, 887 \mathrm{~m}$, 22.08.-01.10.2013, Kalashian leg. / (soil traps Ser.4)"; 1 \%: "ARMENIA, Lori prov., Teghut mine, N41.0967" E44.8218 $, 920 \mathrm{~m}, 20.06 .-01.08 .2013$, Kalashian leg. / (soil traps Ser. 2)"; $10^{\text {t: }}$ "ARMENIA - Teghut env. (Akhtala), $41.091^{\circ} \mathrm{N}, 44.813^{\circ} \mathrm{E}, 1020 \mathrm{~m}, 20 . \mathrm{V} .2016$, leg. A. \& J. Müller" (cAss); 1 \%: “T6 BF Armenia, near Teghut (Akhtala) $1000 \mathrm{~m}$ a. s. 1., N 41.09099 E 44.81202 26.VIII.2016 leg. J. Müller" (cSch); 1 ơ, 1 ㅇ: "ARMENIA - Lori prov., Teghut mine, N41.091 ${ }^{\circ} \mathrm{N}, \mathrm{E} 044.812^{\circ}, 990 \mathrm{~m}$, 13.VIII.-13.X.2017, leg. Kalashian et al." (cAss); 1 \% : 
"ARMENIA - Lori prov., Teghut mine, N41.096 $\mathrm{N}$, E044.822 ${ }^{\circ}, 910$ m, 25.V.-23.VII.2017, leg. Kalashian et al." (cAss); 2 ㅇ ㅇ: "ARMENIA - Lori prov., Teghut mine, $\mathrm{N} 41.091^{\circ} \mathrm{N}, \mathrm{E} 044.851^{\circ}, 890 \mathrm{~m}, 28 . I V .-25 . V .2017$, leg. Kalashian et al." (cAss); 1 ex.: "Lenkoran Leder (Reitter / Coll. Reitter / Anotylus hamatus (Fairm. \& Lab.) det. Makranczy, 1997 / misidentification ! Anotylus sp. n. det. Makranczy, 1998” (HNHM).

Etymology: The specific epithet alludes to the strong resemblance to A. hamatus (FAIRMAIRE \& LABOULBÈnE, 1856), most likely the sister taxon.

Description: Body length 1.8-2.2 mm; length of forebody $1.0-1.2 \mathrm{~mm}$. Coloration: whole body black; legs yellow; antennae black; mandibles and maxillary palpi dark brown.

Measurements: males: head width $0.47-0.50 \mathrm{~mm}$, head length $0.32-0.33 \mathrm{~mm}$, eye length $0.14 \mathrm{~mm}$, length of temples $0.13-0.14 \mathrm{~mm}$, pronotum width $0.49-0.51 \mathrm{~mm}$, pronotum length $0.35 \mathrm{~mm}$, sutural length of elytra $0.36-0.38 \mathrm{~mm}$, elytral width $0.61-0.64 \mathrm{~mm}$. females: head width $0.37-0.38 \mathrm{~mm}$, head length $0.24-0.25 \mathrm{~mm}$, eye length $0.11-0.12 \mathrm{~mm}$, length of temples $0.08 \mathrm{~mm}$, pronotum width $0.40-0.42 \mathrm{~mm}$, pronotum length $0,28-$ $0.29 \mathrm{~mm}$, sutural length of elytra $0.32-0.35 \mathrm{~mm}$, elytral width $0.51-0.53 \mathrm{~mm}$.

In size, body shape, punctuation, and microsculpture indistinguishable from A. hamatus and similar species.

$\sigma^{*}$ : head distinctly larger, transverse (width/length 1.451.57), eyes as long as, or slightly longer than temples (eye length/temple length 1.0-1.1); sternite VII (Figs 47-48) similar to that of $A$. hamatus, with prominent spine and a small tubercle with two larger spines in front of it; sternite VIII highly modified (Figs 49-50), in general respect similar to that of $A$.hamatus, with broad and deep emargination at base, broad emargination at apical border, interrupted in the middle by a short two-lobed process with 4-5 setae at apex of each lobe; aedeagus (Figs 51-52), $0.47 \mathrm{~mm}$ long, nearly symmetric, elongate, with the apical flag-like part of the paramere more extended than in A. hamatus.

\% : head distinctly smaller, transverse (width/length 1.471.59), eyes distinctly longer than temples (eye length/ temple length 1.45-1.50).

Comparative notes: Anotylus hamatoides is distinguished from all other representatives of the Anotylus tetracarinatus group with the outer edge of the front tibiae apically strongly indented only by the male primary and secondary sexual characters. The pronounced similarity to the widespread $A$. hamatus in the shape of the male sternites VII and VIII and in the shape of the aedeagus suggests a close relationship; most likely they represent sister taxa. Both species are distinguished especially by the shape of the apical margin of the male sternite VIII. Differences in the shape of sternite VII and the aedeagus are less pronounced, but constant.
Distribution and natural history: The species is currently known from the environs of Teghut in Lori province, North Armenia, and from the Lenkoran region in Azerbaijan. The Armenian specimens were collected with pitfall traps at elevations between approximately 890 and $1100 \mathrm{~m}$.

\section{Platystethus cephalotes EPPELSHEIM, 1878, revalidated (Figs 53-54)}

Platystethus oblongopunctatus RouBAL, 1911: 2; syn. nov.

A specimen close to Platystethus laevis MärKel \& KIESENWETTER, 1848 with the following labels was found in the Khnzorian collection: "Gegharkunik province, Sevan, Uchtapalar, $3000 \mathrm{~m}$ [= Uch-taplar Mt., 40²4'58.1"N, $45^{\circ} 01^{\prime} 41.3^{\prime \prime E}$, highest 2500, not $3000 \mathrm{~m}$ ], 1.VIII.1948 / F' de Fontainebl. f.[illegible] Gd. Verneur S. \& M. 15.5.1937 [overleaf]". The specimen had been identified as Platystethus cephalotes by Peter Hammond, who never published this record or notes about the taxonomic status of this species. Platystethus cephalotes was described based on material from Michailovo [= Khashuri], a town in the Kura valley in Central Georgia [altitude approximately $750 \mathrm{~m}$, but surrounded by mountain ranges of up to $2300 \mathrm{~m}$ ]. The species was synonymized with P. laevis, a species originally described from the Austrian Alps, by GANGLBAUER (1895), who was the first to record P. laevis from the Caucasus region. This synonymy was followed by Bernhauer \& Schubert (1911), Herman (2001), Smetana (2004), and Schülke \& Smetana (2015). SCHEERPELTz (1955) and Horion (1963) reported P. laevis from the Caucasus region without explicitly referring to P. cephalotes as a junior synonym. The identification by Peter Hammond raised the question if specimens of P. laevis from the Caucasus region and those from Central Europe were really conspecific.

A comparison of specimens identified as P. laevis both from Central Europe (Austria, Italy, Slovenia) and the Caucasus region (Russia: Krasnodar region, Georgia: Abkhasia, Kvemo Svaneti) shows no significant differences in size, body shape, punctation and microsculpture. The same is true of the shape of the aedeagus. The male sternite VIII of Caucasian specimens is slightly more densely punctate in the median subapical region and has longer stout setae on either side of the midline (Fig. 53; for comparison see Fig. 55). The only clear differences between Central European and Caucasian specimens are the shape and especially the apical margin of the male tergite X. In Central European specimens, the tergite is as broad as long, with a more or less truncate and unmodified apical margin (Fig. 56), whereas in Caucasian specimens the tergite is longer than broad, with the apical margin rounded and equipped with a small tooth on either side (Fig. 54). Even though the differences are not pronounced, they are constant, suggesting that the populations from Central Europe and those from 
the Caucasus region represent distinct species. Therefore, $P$. cephalotes is revalidated and P. oblongopunctatus RoubaL, 1911, originally described based on material from Krasnaya Polyana in the Western Caucasus (Russia) and previously a synonym of P. laevis, is synonymized with $P$. cephalotes.

The presence of both species in Armenia is doubtful. The specimen examined from the Khnzorian collection belongs to P. laevis, not to P. cephalotes. It can inferred from the different records on both sides of the locality label, from the absence of $P$. laevis in examined Caucasian material, and finally from the absence of suitable habitats in the surroundings of Paris (France) that the specimen was most likely mislabeled. In consequence, neither of the two species is included in the checklist (Tab. 1).

\subsubsection{Scydmaeninae (by HeInRICH MeYBoHm)}

\section{Euconnus (Cladoconnus) Ialvarensis IABLOKOFF- KHNZORIAN, 1964}

Euconnus lalvarensis IABLOKOFF-KHNZORIAN, 1964a: 154. Euconnus pseudorobustus Franz, 1986: 41; syn. nov.

Type material examined: Holotype $\sigma^{*}$ : "Alaergi Shamlug [Lalvar Shamlugh] ASSR 6.6.60 / Euconnus lalvarensis Iablokoff-Khnzorian / Holotypus Gen. präp. Meybohm 2018” (cKhn).

Comment: Euconnus pseudorobustus was previously known from Georgia and the Turkish province Rize. An examination of the holotype of E. lalvarensis, which was collected in Armenia, in a slope of Lalvar toward Shamlugh, in a dense forest, by sweeping [...], 6.VI.1960 (IABLOKOFF-KhNZORIAN 1964a), revealed that it is conspecific with E.pseudorobustus. The comparison is based on numerous additional specimens from Georgia and Armenia, and on HLAváč \& STEvanović (2013).

\section{Euconnus (Tetramelus) longilaminatus MeYвOHM spec. nov. \\ urn:Isid:zoobank.org:act:96692EB3-A82F-4ADB -A278-EC1B64040C82 (Fig. 57)}

Type material: Holotype $\sigma^{*}$ : "ARMENIA [30] - pass road E Ijevan, $1790 \mathrm{~m}, 40^{\circ} 52^{\prime} 00^{\prime} \mathrm{N}, 45^{\circ} 13^{\prime} 19^{\prime \prime} \mathrm{E}$, forest, litter \& roots sift., 6.VII.2017, V. Assing / Euc. (Tetramelus) longilaminatus m. Meybohm 2018 det. / Holotypus" (cMey). Paratypes: $5 o^{\star} o^{\star}, 5$ 우 우: same data as holotype (cMey).

Etymology: The specific epithet (adjective) alludes to the long ventral process of the aedeagus.

Description: Body length sexually dimorphic, in $o^{7} 1.38$ $1.45 \mathrm{~mm}$ and in $91.48-1.53 \mathrm{~mm}$. Coloration uniformly bright reddish-brown, appendages paler. Pubescence (pronotum excluded) rather sparse, directed posteriad, sub-erect, and bent towards body. Punctation of head and pronotum very fine, that of elytra fine.

Head approximately $0.26 \mathrm{~mm}$ broad, broadest across the minute eyes, weakly oblong, slightly more than 1.1 times as long as broad, lateral contours smoothly curved from the eyes to the posterior constriction, postero-medially weakly bulging and projecting beyond posterior constriction. Eyes rudimentary, with only one ommatidium, without pigmentation, not projecting from lateral contours of head. Antenna with distinct four-jointed club, weakly sexually dimorphic: in $\sigma^{\top}$ antenna approximately $0.76 \mathrm{~mm}$ long and 0.9 times as long as elytra, length of club approximately as long as combined length of antennomeres II-VII; i antenna approximately $0.73 \mathrm{~mm}$ long and 0.8 times as long as elytra, length of club distinctly shorter than $(0.94 \mathrm{x})$ combined length of antennomeres II-VII; antennomeres II twice as long as broad, little shorter than combined length of III and IV, III-VI elongate, III and IV of equal length, V longer and VI shorter than IV, VII intermediate between VI and VIII, 1.3 times as long as broad, from VIII to XI gradually broader and XI slightly shorter than the combined length of IX and X.

Pronotum 1.1-1.2 times as broad as head and as long as broad or slightly longer, broadest slightly anterior to middle, more strongly tapering anteriad than posteriad; base with four pits, internal pits shallowly delimited, separated from each other by approximately three times their diameter, connected by a shallow transverse impression.

Elytra on average of $0.55 \mathrm{~mm}$ ( $o^{*}$ ) or $0.60 \mathrm{~mm}$ ( ( ) broad, in both sexes about 1.5 times as long as broad, broadest behind anterior two-fifths of elytral length, moderately convex in lateral and in dorsal view; each elytron with two clearly separated basal pits, the external one larger and laterally delimited by a short elevation, the internal one delimited by an oblique edge directed towards suture; setae approximately as long as antennomere II. Hind wings completely reduced. Femora and tibia without sexual dimorphism. Femora dilated in distal halves, profemora more strongly dilated than meso- and metafemora. Tibiae straight. Metaventrite medially flat $\left(\sigma^{4}\right)$ or weakly convex in cross-section ( 9 ). Aedeagus shaped as in Fig. 57.

Comparative notes: See section on E. karabakhus.

Distribution and natural history: See section on E. karabakhus.

\section{Euconnus (Tetramelus) tavushus MeYвoнm spec. nov. urn:Isid:zoobank.org:act:7F2BBDD70-A7F8-4272-8195-02884B90F2DD (Fig. 58)}

Type material: Holotype $0^{*}$ : "ARMENIA [26a] - ENE Dilijan, Hovk, 1290 m, 4047'39"N, 4501'17"E, stream 
valley, sifted, 3.VII.2017, V. Assing / Euc. (Tetramelus) tavushus m. Meybohm 2018 det. / Holotypus" (cMey). Paratypes: 2 우 ㅇ: same data as holotype (cMey); $7 o^{\star} o^{\star}, 10$ ㅇ ㅇ : "ARMENIA [17-26a] - ENE Dilijan, Hovk, $1290 \mathrm{~m}, 40^{\circ} 47^{\prime} 39^{\prime \prime} \mathrm{N}, 45^{\circ} 01^{\prime} 17^{\prime \prime} \mathrm{E}$, moist litter near stream sifted, 3.VII.2017 Schülke” (cSch).

Etymology: The specific epithet is an adjective derived from Tavush, the name of the province where the type locality is situated.

Description: External characters as in E. longilaminatus, except as follows:

Body length 1.40-1.45 mm ( $\left.\sigma^{\star}\right)$ and 1.41-1.50 mm ( 9 ). Head approximately $0.24 \mathrm{~mm}$ broad, 1.1 times as long as broad. Eyes slightly projecting from lateral contours of head.

Male antenna $0.78 \mathrm{~mm}$ long on average, 0.92 times as long as elytron, club 0.9 times as long as combined length of antennomeres II-VII; female antenna $0.74 \mathrm{~mm}$ long on average, 0.86 times as long as elytron, club 0.9 times as long as combined length of antennomeres II-VII; antennomeres III, IV and VI of equal length, V longer than IV, VII 1.5 times as long as broad.

Pronotum 1.2 times as broad as head and 1.1 times as long as broad; base with five pits, the internal ones connected by a shallow impression and separated from each other by approximately half their diameter.

Elytra on average $0.55 \mathrm{~mm}$ ( $\sigma^{\star}$ ) or $0.59 \mathrm{~mm}$ ( $($ ) broad, in lateral and in dorsal view moderately convex.

Aedeagus shaped as in Fig. 58.

Comparative notes: See section on E. karabakhus.

Distribution and natural history: See section on E. karabakhus.

\section{Euconnus (Tetramelus) karabakhus MeYвонm spec. nov. \\ urn:Isid:zoobank.org:act:A60B66A1-20EB-4341-9950-B6485B1A3853 (Fig. 59)}

Type material: Holotype $0^{*}$ : "NAGORNO-KARABAKH - [10], NW Stepanakert, 4001'34"N, 46²8'33"E, 1210 m, mixed forest, 3.VII.2018, V. Assing / Euc. (Tetramelus) karabakhus m. Meybohm 2018 det. / Holotypus" (cMey). Paratypes: + : same data as holotype (cMey); 1 ㅇ: same data, but leg. Schülke (cSch); 1 \%: "NAGORNO-KARABAKH - [7], NW Stepanakert, 3954'22"N, 46³9'12"E, $870 \mathrm{~m}$, mixed forest, 2.VII.2018, V. Assing" (cMey); 1 ㅇ: same data, but leg. Schülke (cSch); 1 \%: "NAGORNOKARABAKH [AR 18-11], NW Stepanakert, W Vank, $40^{\circ} 01^{\prime} 44^{\prime \prime} \mathrm{N}, 46^{\circ} 30^{\prime} 15^{\prime \prime} \mathrm{E}, 1080 \mathrm{~m}$, mixed forest, litter sifted, 3.VII.2018, leg. M. Schülke” (cSch).

Etymology: The specific epithet is an adjective derived from Nagorno-Karabakh.
Description: External characters as in E. tavushus, except as follows:

Body length $1.54 \mathrm{~mm}\left(o^{\star}\right)$ and $1.54-1.61 \mathrm{~mm}$ ( + ). Body unicoloured dark reddish-brown.

Head approximately $0.26 \mathrm{~mm}$ broad, slightly more than 1.1 times as long as broad. Eyes slightly projecting from lateral contours of the head.

Antenna $0.79 \mathrm{~mm}$ long on average, about 0.85 times as long as elytra, club about 0.85 times as long as combined length of antennomeres II-VII; III, IV and VI of equal length, V longer than IV, VII 1.7 times as long as broad. Pronotum 1.2 times as broad as head, slightly longer than broad; base with four pits, internal pits distinctly delimited, separated from each other by approximately three times their diameter, connected by a very shallow transverse impression.

Elytra $0.60 \mathrm{~mm}$ (male) and $0.65 \mathrm{~mm}$ (female) broad on average, in lateral and in dorsal view strongly convex. Aedeagus shaped as in Fig. 59.

Comparative notes: The three species described above belong to the Euconnus (Tetramelus) reitteri group (VÍT \& Hlaváč 1998). They differ in body length and the structure of the antennae, the shape of the eyes, the basal pits of the pronotum, and the shape of the aedeagus. The aedeagi of E. tavushus and E. karabakhus are very similar, though broader in E. tavushus than in E. karabakhus, but these species differ significantly in external features.

Distribution and natural history: The ten previously described species of the E. reitteri group have more or less restricted distributions in Northeast Turkey and in Georgia. The distributions of the three species described above extend the general distribution of this group to the east, south, and southeast. The specimens were sifted from leaf litter at altitudes of 870-1790 m.

\section{Neuraphes (Paraphes) gomarantsus Meүвонм spec. nov. \\ urn:Isid:zoobank.org:act:102F7EEC-B2A5-458F-B271-E3582970195F (Figs 60-62)}

Type material: Holotype o : "ARMENIA [30] - $25 \mathrm{~km}$ S Kapan, Gomarants Ps., 3901'32"N, 46²21'59"E, 2190 m, oak forest, 7.VII.2016, V. Assing / Neuraphes gomarantsus m. Meybohm 2018 det. / Holotypus" (cMey). Paratypes: 2 우: same data as holotype (cMey); 1 ㅇ: "ARMENIA [31] - 25 km S Kapan, Gomarants Ps., 3902'15"N, $46^{\circ} 22^{\prime} 13 " \mathrm{E}, 2050 \mathrm{~m}$, oak forest, 7.VII.2016, V. Assing" (cMey).

Etymology: The specific epithet is an adjective derived from Gomarants Pass, the type locality.

Description: Body length 1.80-1.85 mm. Body unicoloured reddish-brown, glossy. Palpi, antennae, and legs light-brown. Pubescence bright, long, and sub-erect; 
setae about as long as antennomere II. Male habitus as in Fig. 60.

Head $0.28 \mathrm{~mm}$ long and $0.29 \mathrm{~mm}$ broad; occipital constriction about three-fourths as broad as head; vertex convex, with a pair of large, very shallow impressions located between the eyes and delimiting a median trapeziform flat area; supra-antennal tubercles marked, temples pronounced, half as long as diameter of eyes; eyes large, in female weakly convex and coarsely faceted, in male more convex and more distinctly faceted.

Antennae in $+0.84-0.91 \mathrm{~mm}$ long, antennomere I twice as long as broad, II distinctly narrower and slightly shorter than I, twice as long as broad, III and IV distinctly narrower than II and much shorter, 1.5 times as long as broad, V slightly broader and longer than III and IV, VI as broad as V, as long as III and IV, 1.3 times as long as broad, VII much broader than VI, 1.2 times as long as broad; VIII-X (measured without apical conical part): VIII broader than VII, as long as broad, IX broader than VIII, slightly broader than long, X broader than IX, 1.4 times as broad as long, XII slightly shorter than IX-X combined. Antennae in male distinctly longer and more slender than in female, $0.98 \mathrm{~mm}$ long, III-V 1.7 times as long as broad, VI much broader than V, but of equal length, XII much shorter than IX-X combined.

Pronotum elongate in dorsal view, $0.45 \mathrm{~mm}$ long and $0.39 \mathrm{~mm}$ broad, broadest at anterior third, lateral margins strongly rounded in anterior third, distinctly converging in posterior half and slightly concave near the almost rectangular hind angles. Posterior margin weakly arcuate; base with a transverse groove, ending laterally in a shallow depression, medially interrupted by a short longitudinal carina, on both sides of the carina with a large impression; punctures very fine.

Elytra oval, broadest at anterior third, length $1.11 \mathrm{~mm}$, width $0.68 \mathrm{~mm}$; humeral calli marked, forming elongate, longitudinal folds; basal pit on each elytron moderately large, closer situated to scutellum than to humerus; apices of elytra separately rounded; punctures in antero-sutural portion fine and similar to those of head, otherwise coarser; interstices slightly narrower than length of setae.

$0^{*}$ : elytra (Fig. 61) subapically near suture with weak longitudinal elevation, this elevation apically with pit of circular shape separated from the elytral posterior margin by its diameter and from the suture by half its diameter; diameter of this pit equal to width of antennomere VI; anterior margin of this pit with sparse short and straight setae; apical portion of elytra with some long setae directed posteriad. Metaventrite medially with a large and oblong oval impression; impressions not delimited by an elevation and with dense, weakly erect pubescence directed posteriad; pubescence of lateral portions sparser, more erect, and directed obliquely postero-laterad.

Aedeagus $0.35 \mathrm{~mm}$ long and $0.19 \mathrm{~mm}$ broad; shaped as in Fig. 62; vestigial parameres distinct, not reaching middle of median lobe; large sclerites bent dorsad apically in lateral view; the four lobes of distal sclerite of similar size.

Comment: One female not included in the type series, but collected together with the holotype, differs from the type specimens by blackish body, shorter antennae $(0.84 \mathrm{~mm})$, shorter elytra $(1.04 \mathrm{~mm})$, and the shape of the pronotum. It most likely belongs to a different species.

Comparative notes: See section on N. syunikus.

Distribution and natural history: See section on N. syunikus.

\section{Neuraphes (Paraphes) syunikus MeYBohm spec. nov. urn:Isid:zoobank.org:act:2972F28B-0968-4810-B9F5-471E93F97F43 (Figs 63-65)}

Type material: Holotype $\sigma^{*}$ : "ARMENIA [27] - WSW

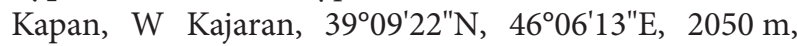
mixed forest, 10.VII.2018, V. Assing / Neuraphes syunikus m. Meybohm det 2018 / Holotypus" (cMey). Paratypes: 9 우: same data as holotype (cMey); 4 우: same data as holotype, but leg. Schülke (cSch); $1 \sigma^{\star}, 1 \%$ : "ARMENIA [AR18-27a] WSW Kapan, W Kajaran, 39 $09^{\prime} 22^{\prime \prime N}$, $46^{\circ} 06^{\prime} 13^{\prime \prime E}, 2050 \mathrm{~m}$, mixed decid. forest, litter/grass roots sifted, 13.VII.2018, leg. M. Schülke" (cSch); 1 \%: "ARMENIA [28] - ca. $30 \mathrm{~km} \mathrm{~W}$ Kapan, $39^{\circ} 15^{\prime} 13^{\prime \prime N}, 46^{\circ} 04^{\prime} 16^{\prime \prime E}$, $2040 \mathrm{~m}$, forest margin, 11.VII.2018, V. Assing" (cMey); 1 9 : "ARMENIA [41a] - pass N Goris, $39^{\circ} 35^{\prime} 34^{\prime \prime} \mathrm{N}$, $46^{\circ} 19^{\prime} 29^{\prime \prime} \mathrm{E}, 1900 \mathrm{~m}$, small stream valleys, 10.VII.2017, V. Assing" (cMey).

Etymology: The specific epithet is an adjective derived from Syunik, the name of the province where the type specimens were found.

Description: External characters as in N. gomarantsus, except as follows:

Body length $1.50-1.57 \mathrm{~mm}$. Body slightly bicoloured: head and pronotum reddish-brown, glossy; elytra darker, brown or black. Male habitus as in Fig. 63.

Head length $0.25 \mathrm{~mm}$, width $0.28 \mathrm{~mm}$; temples moderately pronounced.

Female antenna $0.75-0.79 \mathrm{~mm}$ long, antennomeres I 1.8 times as long as broad, II distinctly narrower and slightly shorter than I, 1.8 times as long as broad, III and IV distinctly narrower and much shorter than II, 1.3 times as long as broad; $\mathrm{V}$ as long as III and IV, but slightly broader, 1.2 times as long as broad, VI as broad as V but shorter, as broad as long, VII much larger than VI, as broad as long, VIII-X (measured without apical conical part) each as long as III, VIII much broader than VII, 1.5 times as broad as long, IX broader than VIII, 1.8 times as broad as long, X broader than IX, twice as broad as long, XII as long as, or slightly shorter than 
IX-X combined. Male antenna $0.82 \mathrm{~mm}$ long, distinctly longer and more slender than in most females. Pronotum $0.45 \mathrm{~mm}$ long and $0.36 \mathrm{~mm}$ broad; base with a transverse groove ending laterally in a distinct pit. Elytra $0.94 \mathrm{~mm}$ long and $0.62 \mathrm{~mm}$ broad; apices of elytra slightly oblique; setae suberect, about as long as antennomere II.

$\sigma^{\star}$ : elytra (Fig. 64) subapically near suture with distinct longitudinal elevation, this elevation posteriorly with a large circular pit separated from apical margin of elytra and from suture by half its diameter; diameter of this pit equal to width of antennomere VII; anterior margin of this impression with dense, very short and straight setae; elytral apices each with an obtuse erect tooth and with conspicuous long setae directed posteriad.

Aedeagus $0.28 \mathrm{~mm}$ long and $0.12 \mathrm{~mm}$ broad, shaped as in Fig. 65; vestigial parameres distinct, reaching middle of median lobe; large sclerites bent ventrad apically; basal lobes of distal sclerite large, apical lobes small.

Comparative notes: Neuraphes syunikus and N. gomarantsus clearly differ in body size, coloration, and particularly in the modifications of the male elytra. Regarding the latter, N. syunikus is most similar to N. caudatus ReITTER, 1896.

Distribution and natural history: The genus is represented in the Caucasus region (including North Turkey) by numerous described and undescribed species, most of which have restricted distributions and are confined to higher altitudes. The two species described above were collected at elevations between 1900 and $2190 \mathrm{~m}$ and may be endemic to South Armenia.

The specimens were sifted from leaf litter, debris, and grass roots in various types of forest and in montane meadows.

\subsubsection{Paederinae (by VoLKeR Assing)}

Astenus rufopacus ReITteR, 1909

Astenus rufopacus ReItTer, 1909: 150.

Astenus baali Coiffait, 1960: 94 f.; syn. nov.

The original description of A. rufopacus is based on an unspecified number of syntypes from "Araxesthal, Lenkoran" (REITTER 1909), that of A. baali on a holotype and several male paratypes from Lebanon, Turkey, and Armenia ("Delizhan" = Dilijan) (CoIffait 1960). Anlaş (2017) studied the type material of A. rufopacus, designated a syntype from Lenkoran as the lectotype, and illustrated the habitus and the male sexual characters. The type material of $A$. baali is deposited in the Coiffait collection in the Natural History Museum in Paris and consequently inaccessible for scientific study (see Assing 2018f). However, based on the details indi- cated in the original description of $A$. baali and the illustrations of the aedeagus (CoIfFAIT 1960: figures 61-62), there is little doubt that the type material of A. baali is conspecific with that of $A$. rufopacus.

\subsubsection{Staphylininae (by VoLKER Assing)}

\section{Heterothops dissimilis (GRAVENHORST, 1802)}

Heterothops armeniacus CoIfFAIT, 1977: 142; syn. nov.

Type material examined: Holotype $q$ : "Erevan, Kamaker, ASSR - 4.11.48 / Holotype / Heterothops armeniacus Coiff., H. Coiffait det. 1969 / Heterothops dissimilis (Gravenhorst), det. V. Assing 2018” (cKhn).

Comment: The original description of $H$. armeniacus is based on a unique female holotype from "Erevan, Kamaker, Arménie soviétique" (CoIffait 1977). In external characters, this holotype is identical to Armenian material identified as $H$. dissimilis. A comparison of the internal structures of the aedeagus of material from Germany and Armenia yielded no evidence that these populations should represent different species. These internal structures are slightly longer in the Armenian specimens, but otherwise of identical shape. Consequently, these differences are attributed to intraspecific variation and $H$. armeniacus is placed in synonymy with H. dissimilis.

\section{Heterothops laeticolor ReITTER, 1891}

Material examined: Armenia: $1 \sigma^{\star}$, Echmiadzin, Markara, 11.VII.1963 (cKhn). Azerbaijan: $10^{*}$, Nakhchivan, Dzhulfa, Araks,, 3.V.1955 (cKhn).

Comment: In external characters, this species is similar to H. minutus Wollaston, 1860, from which it differs only by slight, but distinct differences in the shapes of the internal structures of the aedeagus.

\section{Heterothops microtophilus CoIfFAIT, 1977}

Material examined: Armenia: $2 \sigma^{\star}$, Gukasyan, $2000 \mathrm{~m}$, Microtus arvalis, 3.I.1967 (cKhn).

Comment: In the shape of the head and the blackish coloration, this species somewhat resembles $H$. praevius, from which it differs by smaller body size (length of forebody 1.9-2.0 mm), completely blackish elytra, more or less distinctly paler basal antennomeres, and by internal structures of the aedeagus of completely different shape. 
Heterothops praevius ERICHSON, 1839

Heterothops montanus IABlokoff-Khnzorian, 1966: 174; syn. nov.

Type material examined: Paratypes: $1 \sigma^{\star}, 1$ ㅇ: "Aragaz. 3000, Karilch [= K’ari Lich lake], ASSR. 29.8.48 / vue C / Paratypus / montanus / Heterothops praevius Erichson, det. V. Assing 2018” (cKhn).

Comment: The original description of H. montanus is based on several type specimens from Mount Aragats (IABlokoff-Khnzorian 1966). An examination of the aedeagus (including the internal structures) of the male paratype above revealed that it is identical to that of $H$. praevius. Hence the synonymy proposed above.

\section{Rabigus abauriae (GRIDELLI, 1924)}

The Armenian record of Rabigus tenuis (FABRICIUs, 1782) in EICHLER (1930) most likely refers to R. abauriae. Similarly, a revision of a female from Amasya, which the sole record of $R$. tenuis from Turkey (Assing 2009b) is based on, revealed that it belongs to $R$. abauriae. Consequently, $R$. tenuis is currently unknown from Turkey and Armenia.

\section{Acknowledgements}

The present monograph would not have been possible in the present form without the help of numerous colleagues, particularly those that helped with the identification of a substantial number of species: Volker Brachat (nearly all Pselaphinae), Johannes Frisch, MNB (most Scopaeus spp.), Mikhail Gildendov, Smolensk (Thinodromus dilaticollis), Ivan Löbl, Genève (Scaphisoma cf. agaricinum), Heinrich Meybohm (Scydmaeninae), Volker Puthz (some Steninae), Alexey Shavrin, Daugavpils (Anthobium unicolor, some Geodromicus spp., Lesteva monticola), Heinrich Terlutter, Münster (some Ocalea spp.), Jürgen Vogel, Görlitz (numerous Athetini), Adriano Zanetti (Dropephylla spp., Eusphalerum spp., some Omalium spp., Olophrum caucasicum). The following colleagues revised the literature references of certain taxa: Volker Brachat (Pselaphinae), Johannes Frisch (Scopaeus spp.), György Makranczy, Budapest (Ochthephilus spp.), Heinrich Meybohm (Scydmaeninae), Volker Puthz (Steninae), Harald Schillhammer (Staphylinini), Alexey Shavrin (Omaliinae), Adriano Zanetti (Omaliinae). Helpful information on localities was contributed by Alexandr Miroshnikov (Krasnodar), Maxim Nabozhenko (Moscow), Alexey Shavrin (Daugavpils), Alexey Solodovnikov (Copenhagen), and Tigran Ghrejyan (Yerevan). Additional records were provided by Volker Puthz (Steninae), Harald Schillhammer (Staphylininae),
Alexey Shavrin (Geodromicus spp.), and Adriano Zanetti (Omaliinae). Important material was made available by Tigran Ghrejyan, Mark Kalashian, Matúš Kocian, Jörg Müller (Grafenau), and Harald Schillhammer. Support and guidance during our field trips was perfectly organized by Tigran Ghrejyan, Mark Kalashian, and Gayane Karagyan. Tigran Ghrejyan also provided us with helpful literature, on-the-spot expertise, and entertainment in various respects. Benedikt Feldmann (Münster), Harald Schillhammer, and Adriano Zanetti proof-read and reviewed the manuscript; their suggestions and corrections helped to improve the manuscript in various ways.

\section{References}

Amiryan, A. L. 1999a: Obzor predstavitelei roda Tachinus Grav. (Coleoptera, Staphylinidae) Armenii. [The scetch (sic) of the genus Tachinus (Coleoptera, Staphylinidae) from Armenia]. - National Academy of Sciences of Armenia, Collection of articles of the young researchers: 95-98.

Amiryan, A. L. 1999b: Novy vid Aleochara Gravenhorst is Armenii (Coleoptera, Staphylinidae). - Biological Journal of Armenia 52 (1): 62-63.

Amiryan, A. L. 2000: The fauna of staphylinid beetles (Coleoptera, Staphylinidae) in Armenia (PhD thesis, concise version). - Laboratory of Entomology \& Soil Zoology, Institute of Zoology, National Academy of Sciences, Armenia: 23 pp.

Amiryan, A. L.; Telnov, D.; Hlaváč, P. \& Willers, J. 2000: To the knowledge of pholeophilic, nidicolic and myrmecophilic staphylinid - beetles [sic] (Coleoptera, Staphylinidae) from Armenia. - Proceedings of the Republican Youth Scientific Conference, Yerevan: 7-15.

Anlaş, S. 2017: Notes on the genus Astenus Dejean, 1833 from the Palearctic region (Coleoptera: Staphylinidae: Paederinae). - Türkiye Entomoloji Dergisi 41 (4): 405-413. - http://dergipark.gov.tr/download/articlefile/451101.

Anlaş, S. 2018: Notes on the genera Paederidus Mulsant \& Rey, 1878, Paederus Fabricius, 1775 and Uncopaederus Korge, 1969 from the Palearctic region (Coleoptera: Staphylinidae: Paederinae). - Türkiye Entomoloji Dergisi 42 (4): 3-12. - http://dergipark. gov.tr/download/article-file/453457.

Anlaş, S.; Khachikov, E. A. \& Özgen, I. 2011: On the genus Achenium LEACH, 1819 from Turkey and adjacent regions (Coleoptera: Staphylinidae: Paederinae). - Zoology in the Middle East 54 (1): 144-146. - DOI: 10.1080/09397140.2011.10648890.

Assing, V. 1996: A revision of the European species of Calodera Mannerheim (Coleoptera, Staphylinidae, Aleocharinae). - Beiträge zur Entomologie, Berlin 46 (1): 3-24. - https://www.contributions-to-entomo logy.org/article/view/1424/1423. 
Assing, V. 1997: A revision of Othius Stephens, 1829. III. The species of the Western Palaearctic region exclusive of the Atlantic Islands (Coleoptera, Staphylinidae: Xantholininae). - Nova Supplementa Entomologica, Berlin 10: 3-130.

Assing, V. 1999: A revision of the genus Piochardia Heyden, 1870 (Insecta: Coleoptera: Staphylinidae: Aleocharinae). - Annalen des Naturhistorischen Museums in Wien $101 \mathrm{~B}:$ 277-301. - https://www. zobodat.at/pdf/ANNA_101B_0277-0301.pdf.

Assing, V. 2002: A taxonomic and phylogenetic revision of Amarochara Thomson. I. The species of the Holarctic region (Coleoptera: Staphylinidae, Aleocharinae, Oxypodini). - Beiträge zur Entomologie, Keltern 52 (1): 111-204. - https://www.contributions-toentomology.org/article/view/1559/1558.

Assing, V. 2003: A revision of Othiini. XIII. Horizontal and vertical distribution of Othius, new species, and additional records (Coleoptera: Staphylinidae: Staphylininae). - Entomological Problems 33 (1-2): 69-88.

Assing, V. 2004a: A revision of the Medon species of the Eastern Mediterranean and adjacent regions (Insecta: Coleoptera: Staphylinidae: Paederinae). - Bonner Zoologische Beiträge 52 (1-2): 33-82. - https://www.zobodat.at/pdf/Bonner-ZoologischeBeitraege_52_0033-0082.pdf.

Assing, V. 2004b: New species and records of Staphylinidae from Turkey III (Insecta: Coleoptera). Linzer Biologische Beiträge 36 (2): 669-733. - https:// www.zobodat.at/pdf/LBB_0036_2_0669-0733.pdf.

Assing, V. 2005a: On the types of some species of Staphylinidae described by G. Luze; S. M. IAblokofFKhnzorian, and H. Franz (Insecta: Coleoptera). - Entomologische Blätter 101 (1): 57-64.

Assing, V.2005b: A revision of the genus Leptobium CASEY (Coleoptera: Staphylinidae: Paederinae). - Stuttgarter Beiträge zur Naturkunde, Serie A (Biologie), Nr. 673: 1-182. - https://www.naturkundemuseum-bw.de/ sites/default/files/publikationen/serie-a/A673.pdf.

Assing, V. 2006: On the taxonomy and biogeography of Stenus (s. str.) erythrocnemus Eppelsheim and related species (Insecta: Coleoptera: Staphylinidae). - Bonner Zoologische Beiträge 53 (3-4) (2004): 303-310.

Assing, V. 2007a: Proteinus crenulatus - a complex of five species (Coleoptera: Staphylinidae: Proteininae). - Beiträge zur Entomologie, Keltern 57 (2): 355-366. - https://www.contributions-to-entomology.org/ article/view/1709/1708.

Assing, V. 2007b: A revision of the species of Pronomaea ERICHSON of the Western Palaearctic region, including Middle Asia (Coleoptera: Staphylinidae: Aleocharinae: Pronomaeini). - Beiträge zur Entomologie, Keltern 57 (2): 367-396. - https://www.contributions-toentomology.org/article/view/1710/1709.
Assing, V. 2008a: A revision of the Micrillus species of the Palaearctic region, with notes on two species from adjacent parts of the Afrotropical and Oriental regions (Coleoptera: Staphylinidae: Paederinae). - Stuttgarter Beiträge zur Naturkunde Serie A, Neue Serie 1: 301-344. - https://www.naturkundemuseum-bw. $\mathrm{de} /$ sites/default/files/publikationen/serie-a/ans0109assing.pdf.

Assing, V. 2008b: On the taxonomy and zoogeography of some Palaearctic Paederinae and Xantholinini (Coleoptera: Staphylinidae). - Linzer Biologische Beiträge 40 (2): 1237-1294. - https://www.zobodat. at/pdf/LBB_0040_2_1237-1294.pdf.

Assing, V. 2008c: A revision of the Sunius species of the Western Palaearctic region and Middle Asia (Coleoptera: Staphylinidae: Paederinae). - Linzer Biologische Beiträge 40 (1): 5-135. - https://www. zobodat.at/pdf/LBB_0040_1_0005-0135.pdf.

Assing, V. 2009a: On the taxonomy and zoogeography of some Palaearctic Aleochara species of the subgenera Xenochara Mulsant \& Rey and Rheochara Mulsant \& Rey (Coleoptera: Staphylinidae: Aleocharinae). Beiträge zur Entomologie, Keltern 59 (1): 33-101. - https://www.contributions-to-entomology.org/ article/view/1741/1740.

Assing, V. 2009b: On the Staphylinidae of Turkey. VI. Thirteen new species and additional records (Coleoptera). - Koleopterologische Rundschau 79: 117-172. - https://www.zobodat.at/pdf/KOR_79_ 2009_0117-0172.pdf.

Assing, V. 2010a: A revision of the genus Luzea (Coleoptera: Staphylinidae: Paederinae). - Deutsche Entomologische Zeitschrift 57 (1): 117-135. - https:// doi.org/10.1002/mmnd.201000011.

Assing, V. 2010b: A revision of Achenium (Coleoptera: Staphylinidae: Paederinae). - Nova Supplementa Entomologica 21: 1-190.

Assing, V. 2012: On the taxonomy and zoogeography of some Oxypoda species of the West Palaearctic region (Coleoptera: Staphylinidae: Aleocharinae). - Linzer Biologische Beiträge 44 (1): 365-399. - https://www. zobodat.at/pdf/LBB_0044_1_0365-0399.pdf.

Assing, V. 2013a: A revision of Palaearctic Medon IX. New species, new synonymies, a new combination, and additional records (Coleoptera: Staphylinidae: Paederinae). - Entomologische Blätter und Coleoptera 109: $233-270$.

Assing, V. 2013b: On the Palaearctic and Oriental species of Scymbalium and Micrillus (Coleoptera: Staphylinidae: Paederinae). - Linzer Biologische Beiträge 45 (2): 1479-1520. - https://www.zobodat. at/pdf/LBB_0045_2_1479-1520.pdf.

Assing, V. 2014: On the Bolitochara species of the West Palaearctic region (Coleoptera: Staphylinidae: Aleocharinae). - Stuttgarter Beiträge zur Naturkunde A, Neue Serie 7: 33-63. - https://www. naturkundemuseum-bw.de/sites/default/files/ publikationen/serie-a/ans07-04.pdf. 
Assing, V. 2015: A revision of Othiini. XIX. Two new species of Othius, the first record of the genus from Vietnam, and additional records (Coleoptera: Staphylinidae: Staphylininae). - Linzer Biologische Beiträge 47 (2): 1235-1250. - https://www.zobodat.at/pdf/ LBB_0047_2_1235-1250.pdf.

Assing, V. 2016a: Revision of the Anaulacaspis species of the Palaearctic region (Coleoptera: Staphylinidae: Aleocharinae). - Contributions to Entomology 66 (2): 201-255. - https://www.contributions-toentomology.org/article/view/1904/1903.

Assing, V. 2016b: A revision of Geostiba of the West Palaearctic region. XXIII. On the Sibiota species of the Caucasus region exclusive of Turkey (Coleoptera: Staphylinidae: Aleocharinae). - Linzer Biologische Beiträge 48 (2): 1097-1117. - https://www.zobodat. at/pdf/LBB_0048_2_1097-1117.pdf.

Assing, V. 2016c: New species of Oxypoda from Armenia and Georgia (Coleoptera: Staphylinidae: Aleocharinae). - Linzer Biologische Beiträge 48 (2): 1119-1136. - https://www.zobodat.at/pdf/LBB_ 0048 _2_1119-1136.pdf.

Assing, V. 2016d: On some species of the Quedius obliqueseriatus group, with notes on Q. nivicola (Coleoptera: Staphylinidae: Staphylininae). - Linzer Biologische Beiträge 48 (2): 1137-1148. - https:// www.zobodat.at/pdf/LBB_0048_2_1137-1148.pdf.

Assing, V. 2017a: A revision of types and additional material of Staphylinidae from the IablokoffKhnzorian collection (Insecta: Coleoptera). - Linzer Biologische Beiträge 49 (1): 333-340. - https://www. zobodat.at/pdf/LBB_0049_1_0333-0340.pdf.

Assing, V. 2017b: On the Geostiba fauna of Armenia (Coleoptera: Staphylinidae: Aleocharinae). - Linzer Biologische Beiträge 49 (2): 1075-1092. - https:// www.zobodat.at/pdf/LBB_0049_2_1075-1092.pdf.

Assing, V. 2017c: On the Leptusa fauna of the Caucasus region (Coleoptera: Staphylinidae: Aleocharinae). - Linzer Biologische Beiträge 49 (2): 1049-1074. - https://www.zobodat.at/pdf/LBB_0049_2_10491074.pdf.

Assing, V. 2017d: A revision of Palaearctic Lobrathium. VI. Two new species from Armenia and Taiwan, and additional records (Coleoptera: Staphylinidae: Paederinae). - Linzer Biologische Beiträge $49(1)$ : 299-306. - https://www.zobodat.at/pdf/LBB_0049_ 1_0299-0306.pdf.

Assing, V. 2017e: On the micropterous Quedius (Raphirus) species with a punctate scutellum of Turkey (Coleoptera: Staphylinidae: Staphylininae). - Linzer Biologische Beiträge 49 (2): 1029-1039. - https:// www.zobodat.at/pdf/LBB_0049_2_1029-1039.pdf.

Assing, V. 2017f: Taxonomic and faunistic notes on some West Palaearctic and Middle Asian Xantholinini, with a revalidation and new synonymies (Coleoptera: Staphylinidae: Staphylininae). - Linzer Biologische Beiträge 49 (1): 235-252. - https://www.zobodat.at/ pdf/LBB_0049_1_0235-0252.pdf.
Assing, V. 2017g: A revision of Sunius XVI. Two new species from Iran and Iraq (Coleoptera: Staphylinidae: Paederinae). - Contributions to Entomology 67 (2): 247-253. - https://www.contributions-toentomology.org/article/view/1940/1942.

Assing, V. 2018a: On the Aleochara subgenera Ceranota and Xenochara. IV. A revision of types, a new species, and additional records (Coleoptera: Staphylinidae: Aleocharinae). - Linzer Biologische Beiträge 50 (1): 129-148. - https://www.zobodat.at/pdf/LBB_0050_ 1_0129-0148.pdf.

Assing, V. 2018b: A new Atheta species from the West Caucasus and a redescription of A. brevapicalis (Coleoptera: Staphylinidae: Aleocharinae). - Linzer Biologische Beiträge 50 (1): 67-74. - https://www. zobodat.at/pdf/LBB_0050_1_0067-0074.pdf.

Assing, V. 2018c: Three new species of Oxypoda from Spain, Armenia, and Ukraine, with notes on the fauna of Armenia (Coleoptera: Staphylinidae: Aleocharinae). - Linzer Biologische Beiträge 50 (1): 111-127. - https://www.zobodat.at/pdf/LBB_0050 1_0111-0127.pdf.

Assing, V.2018d:A revision of Medon.XI. Fivenew species, additional records, and the first confirmed records from the Oriental region (Coleoptera: Staphylinidae: Paederinae). - Contributions to Entomology 68 (1): 69-81. - https://www.contributions-to-entomology. org/article/view/1961/1979.

Assing, V. 2018e: Revision of the Cousya species of the West Palaearctic Region (Coleoptera: Staphylinidae: Aleocharinae). - Stuttgarter Beiträge zur Naturkunde A, Neue Serie 11 (in press). - https://doi.org/10.18476/ insy.v01.a9.

Assing, V. 2018f: On the taxonomy and zoogeography of some West Palaearctic Quedius species, with a focus on the East Mediterranean and the species allied to Quedius umbrinus and Q. nivicola (Coleoptera: Staphylinidae: Staphylininae). - Linzer Biologische Beiträge 50 (1): 129-182.

Assing, V. 2018g: On some Myllaena species in the East Mediterranean and Caucasus regions (Coleoptera: Staphylinidae: Aleocharinae). - Linzer Biologische Beiträge 50 (2): 1015-1032. - https://www.zobodat. at/pdf/LBB_0050_2_1015-1032.pdf.

Assing, V. 2018h: A revision of Geostiba of the West Palaearctic region. XXVI. New species and additional records, primarily from the Caucasus region (Coleoptera: Staphylinidae: Aleocharinae). - Linzer Biologische Beiträge 50 (2): 1033-1054. - https://www.zobodat.at/pdf/LBB_0050_2_10331054.pdf.

Assing, V. 2019a: On the Lomechusini fauna of the Palaearctic and Oriental regions. XXVI. New species, a new synonymy, and additional records (Coleoptera: Staphylinidae: Aleocharinae). - Contributions to Entomology 69 (1): 33-70. 
Assing, V. 2019b: On the taxonomy of some West Palaearctic Quedius species, with descriptions of new species and new synonymies (Coleoptera: Staphylinidae: Staphylininae). - Linzer Biologische Beiträge 51 (1) (in press).

Assing, V. 2019c: A revision of the Alevonota species of the Palaearctic region. III. Two new species from Taiwan, a new combination, a new synonymy, and additional records (Coleoptera: Staphylinidae: Aleocharinae). Linzer Biologische Beiträge 51 (1) (in press).

Assing, V. \& SCHüLKE, M. 2017: On the Ischnosoma fauna of Georgia (Coleoptera: Staphylinidae: Tachyporinae). - Contributions to Entomology 67 (2): 195-206. https://www.contributions-to-entomology.org/article /view/1937/1933.

Assing, V.; Schülke, M.; Brachat, V. \& Meybohm, H. 2018: On the Staphylinidae of the Greek island Corfu (Insecta: Coleoptera). - Contributions to Entomology 68 (1): 31-67. - https://doi.org/ 10.21248/contrib.entomol.68.1.31-67.

Assing, V. \& Solodovnikov, A. Y. 1998: Three new species of Othius Stephens from the Caucasus (Coleoptera: Staphylinidae: Xantholininae). Zoosystematica Rossica 7: 299-305.

Assing, V. \& Vogel, J. 2017: On some Athetini from Armenia and adjacent regions (Coleoptera: Staphylinidae: Aleocharinae). - Linzer Biologische Beiträge 49 (1): 341-368. - https://www.zobodat.at/ pdf/LBB_0049_1_0341-0368.pdf.

Assing, V. \& Wunderle, P. 2008: On the Alevonota species of the Western Palaearctic region (Coleoptera: Staphylinidae: Aleocharinae: Athetini). - Beiträge zur Entomologie, Keltern 58 (1): 145-189. - https:// www.contributions-to-entomology.org/article/ view/1723/1722.

Benick, G. 1974: Neue und seltene Atheten aus SowjetRussland. - Nouvelle Revue d'Entomologie 4: 25-37.

Benick, G. 1985: Atheta lazorkoi n. sp. (Coleoptera: Staphylinidae) aus der Ukraine. - Revue Suisse de Zoologie 92 (2): 299-302. - https://doi.org/10.5962/ bhl.part.81618.

Bernhauer, M. 1901: Neunte Folge neuer Staphyliniden aus Europa, nebst Bemerkungen. - Verhandlungen der Kaiserlich-Königlichen Zoologisch-Botanischen Gesellschaft in Wien 50: 38-50.

Bernhauer, M. 1908: Beiträge zur Kenntnis der paläarktischen Staphyliniden-Fauna. - Münchner Koleopterologische Zeitschrift 3: 320-335.

Bernhauer, M. 1932: Neuheiten der paläarktischen Staphylinidenfauna. - Koleopterologische Rundschau 17 [1931]: 232-245. - https://www.zobodat.at/pdf/ KOR_17_1931_0232-0245.pdf.

Bernhauer, M. 1944: Übersicht über die paläarktischen Arten der Gattung Actobius FaUv., nebst Beschreibungen zweier neuer Atheta- (Ceritaxa-) Arten (Col. Staphyl.). - Koleopterologische Rundschau 30: 148-150. - https://www.zobodat.at/pdf/KOR_30_ 1944_0148-0150.pdf.
Bernhauer, M. \& Scheerpeltz, O. 1926: Staphylinidae VI. - In: JUnK, W. \& Schenkling, S. (eds.), Coleopterorum Catalogus. Volume 5. Staphylinidae. - Junk, Berlin: 499-988.

Bernhauer, M. \& Schubert, K. 1910: Staphylinidae I. In: Junk, W. \& SchenkLIng, S. (eds.), Coleopterorum Catalogus. Volume 5. Staphylinidae. - Junk, Berlin: $1-86$.

Bernhauer, M. \& Schubert, K. 1911: Staphylinidae II. In: Junk, W. \& Schenkling, S. (eds.), Coleopterorum Catalogus. Volume 5. Staphylinidae. - Junk, Berlin: 87-190.

Bernhauer, M. \& Schubert, K. 1912: Staphylinidae III. In: Junk, W. \& Schenkling, S. (eds.), Coleopterorum Catalogus. Volume 5. Staphylinidae. - Junk, Berlin: 191-288.

Bernhauer, M. \& Schubert, K. 1914: Staphylinidae IV. In: Junk, W. \& Schenkling, S. (eds.), Coleopterorum Catalogus Volume 5. Staphylinidae. - Junk, Berlin: 289-408.

Besuchet, C. \& Kurbatov, S. 2007: Les Bryaxis du Caucase et du secteur oriental des chaînes Pontiques (Coleoptera: Staphylinidae: Pselaphinae). - Russian Entomological Journal 16 (2): 155-206.

Besuchet, C. \& Sabella, G. 1999: Descrizione di nuove specie di Tychus della regione palearctica con revisione dei Tychus del gruppo armeniacus (Coleoptera: Staphylinidae: Pselaphinae). - Annales de la Société Entomologique de France (N.S.) 35 (3-4): 303-318.

Bordoni, A. 1975: Studie sulla sistematica e la geonemia degli Xantholinus. VIII. Le specie eurocentroasiatiche e caucasiche in particolare. Revisione di tipi e descrizione di nuove entità. - Memorie della Società Entomologica Italiana 53 [1974]: 56-96.

Bordoni, A. 1984: Appunti per una revisione dei Geodromicus ReDT. della regione Paleartica Occidentale (Col. Staphylinidae). - Redia 67: 19-59.

Bordoni, A. 2011a: Notes on Palaearctic Xantholinini. VI. New species and new records from Caucasus. - Fragmenta Entomologica 43 (1): 41-56. - DOI: 10.4081/fe.2011.52.

Bordoni, A. 2011b: Redesignations of lectotypes and information on the current location of some types of Staphilinidae [sic]. - Quaderno di Studi e Notizie di Storia Naturale della Romagna 34: 105-110.

Bordoni, A. 2017: New data on the Palaearctic Xantholinini. 14. Species in the Zoological Museum of the Zoological Museum of the Moscow Lomonosov State University (Coleoptera Staphylinidae). - Redia 100: $53-63$.

Cameron, M. 1939: New species of Asiatic Staphylinidae (Col.). - The Entomologist's Monthly Magazine 75: 162-163.

Coiffait, H. 1956: Les Xantholinitae de France et des régions voisines, (Col. Staphylinidae). - Revue Française d'Entomologie 23: 31-75. 
Coiffait, H. 1960: Les Astenus d'Europe et de la méditerrranéenne (Coléoptères, Staphylinidae). Bulletin de la Société d'Histoire Naturelle de Toulouse 95: 49-99.

Coiffait, H. 1965: Les Erichsonius Fauv. (Col. Staphylinidae) d'Europe et de la région méditerranéenne. Description de formes nouvelles. - Annales de la Société Entomologique de France (N. S.) 1: 843-849.

Coiffait, H. 1966a: Nouveaux Philonthini de la région paléarctique occidentale. - Bulletin de la Société d'Histoire Naturelle de Toulouse 102: 506-510.

Coiffait, H. 1966b: Novye Xantholinini iz Sovetskogo Soyuza (Coleoptera, Staphylinidae). - Zoologichesky Zhurnal 45: 195-202.

Coiffait, H. 1967a: Tableau de détermination des Philonthus de la région Paléarctique occidentale (Col. Staphylinidae). - Annales de la Société Entomologique de France (N. S.) 3: 381-450.

Coiffait, H. 1967b: Quedius nouveaux ou mal connus. Bulletin de la Société d'Histoire Naturelle de Toulouse 103 (3-4): 391-424.

Coiffait, H. 1968: Scopaeus nouveaux ou mal connus de la région paléarctique occidentale. - Bulletin de la Société d'Histoire Naturelle de Toulouse 104 (3-4): 405-426.

CoIfFAIt, H. 1969: Une nouvelle espèce du genre Jurecekia RAmbousek. - Bulletin de la Société d'Histoire Naturelle de Toulouse 105 (1-2): 38-40.

Corffait, H. 1970a: Tableau des Stilicus de la région paléarctique occidentale avec description d'une espèce nouvelle (Coléoptères Staphylinidae). - Bulletin de la Société d'Histoire Naturelle de Toulouse 106 (1-2): 146-155.

CoIfFAIT, H. 1970b: Nouveaux Xantholinus paléarctiques. - Bulletin de la Société d'Histoire Naturelle de Toulouse 105 (3-4) [1969]: 287-294.

Coiffait, H. 1970c: Staphylinides nouveaux ou mal connus de la région paléarctique occidentale. Bulletin de la Société d'Histoire Naturelle de Toulouse 106 (1-2): 99-111.

Coiffait, H. 1970d: Formes nouvelles ou mal connues des genres Medon et Hypomedon. - Annales de Spéléologie 24: 701-727.

Coiffait, H. 1972a: Paederinae nouveaux ou mal connus de la région paléarctique occidentale (Coléoptères Staphylinidae). - Nouvelle Revue d'Entomologie 2 (2): 131-150.

Coiffait, H. 1972b: Coléoptères Staphylinidae de la région paléarctique occidentale. I. Généralités, sous-familles: Xantholininae et Leptotyphlinae. Supplément à la Nouvelle Revue d'Entomologie 2 (2): $1-651$.

Coiffait, H. 1974a: Coléoptères staphylinides de la région paléarctique occidentale II. Sous famille Staphylininae, Tribus Philonthini et Staphylinini. - Nouvelle Revue d'Entomologie (Supplément) 4: $1-593$.
Coiffait, H. 1977: Note sur quelques Quedius et Heterothops nouveaux ou mal connus (Col. Staphylinidae). - Nouvelle Revue d'Entomologie 7: 133-143.

Coiffait, H. 1982: Coléoptères Staphylinidae de la région paléarctique occidentale. IV. Sous famille Paederinae. Tribu Paederini 1 (Paederi, Lathrobii). - Nouvelle Revue d'Entomologie, Supplément 12 (4): 1-440.

Davies, A. 2004: Cyrtoscydmini; pp. 206-223. - In: Löbl, I. \& Smetana, A. (eds), Catalogue of Palaearctic Coleoptera. Volume 2. Hydrophiloidea - Histeroidea - Staphylinoidea. - Apollo Books, Stenstrup: 942 pp.

Dvořák, M. 1984: Zur Kenntnis einiger myrmekophiler Staphylinidae (Coleoptera). - Acta Entomologica Bohemoslovaca 81: 190-203.

EIchleR, W. 1930: Chrząszcze okolicy Eczmiadzinu, Erywańkiej gub., Kaukaz. płd. (Zakaukasie). Coléoptères du Gouvernement Eriwan (Caucase méridional). - Polskie Pismo Entomologiczne 8 (1-4) [1929]: 141-183.

Enushchenko, I. V. \& Semenov, V. B. 2016: A review of the genus Gyrophaena MANNERHEIM 1830 (Coleoptera: Staphylinidae: Aleocharinae: Gyrophaenina) of the Caucasus and adjacent territories. - Zootaxa 4126 (3): 301-337. - http://doi.org/10.11646/zootaxa.4126.3.1.

Eppelsheim, E. 1878: Staphylinidae. - In: Schneider, O. \& Leder, H.: Beiträge zur Kenntniss der Kaukasischen Käferfauna. - Verhandlungen des Naturforschenden Vereines in Brünn 16 [1877]: 90-131.

Eppelsheim, E. 1890a: Neue Staphylinen aus den Kaukasusländern. - Wiener Entomologische Zeitung 9: 161-172. - https://www.zobodat.at/pdf/ WEZ_9_0161-0172.pdf.

Eppelsheim, E. 1890b: Neue Staphylinen aus den Kaukasusländern. - Wiener Entomologische Zeitung 9: 217-229. - https://www.zobodat.at/pdf/WEZ_9_ 0217-0229.pdf.

FaldermanN, F. 1835: Additamenta Entomologica ad Faunam Rossicam in itineribus Jussu Imperatoris Augustissimi annis 1827-1831 a Cl. Ménétriés et Szovitz susceptis collecta, in lucem edita. - Nouveaux Mémoires de la Société Impériale des Natiralistes de Moscou 4: 1-310, tab. I-X.

Faldermann, F. 1837: Einige Worte ueber die Fauna Entomologica Transcaucasica. - Bulletin de la Société Impériale des Naturalistes de Moscou 10: 40-43.

Fauvel, A. 1871: Faune Gallo-Rhénane ou descriptions des insectes qui habitent la France, la Belgique, la Hollande, le Luxembourg, les provinces Rhénanes et la Valais avec tableaux synoptiques et planches gravées. - Bulletin de la Société Linnéenne de Normandie (2) 5 [1869-70]: 27-192.

Fauvel, A. 1872: Faune Gallo-Rhénane ou species des insectes qui habitent la France, la Belgique, la Hollande, le Luxembourg, la prusse Rhénane, la Nassau et la Valais avec tableaux synoptiques et planches gravées. Tome 3. Livraison 3. - Le BlancHardel, Caen: 214 pp. 
Fauvel, A. 1874: Faune Gallo-Rhénane ou species des insectes qui habitent la France, la Belgique, la Hollande, le Luxembourg, la prusse Rhénane, la Nassau et la Valais avec tableaux synoptiques et planches gravées. - Bulletin de la Société Linnéenne de Normandie 8 (2): 167-340.

Fauyvush, G. M. \& Aleksanyan, A. S. 2016: Habitats of Armenia. - National Academy of Sciences of the Republic of Armenia, Yerevan: 358 pp.

Franz, H. 1986: Drei neue Euconnus-Arten aus dem Kaukausus (Coleoptera, Scydmaenidae). Zeitschrift der Arbeitsgemeinschaft Österreichischer Entomologen 38: 41-45. - https://www.zobodat.at/ pdf/ZAOE_38_0041-0045.pdf.

FrISCH, J. 1997: A revision of some West Palaearctic species of Scopaeus Erichson (Coleoptera, Staphylinidae, Paederinae). - Revue Suisse de Zoologie 104 (3): 523-557.

FrISCH, J. 2007a: Scopaeus farsensis sp. n. from South Iran, with remarks on S. chalcodactylus (KolenATI, 1846) (Coleoptera, Staphylinidae, Paederinae). - Deutsche Entomologische Zeitschrift 54 (1): 79-88. - https:// doi.org/10.1002/mmnd.200700008.

FrIsCH, J. 2007b: A review of the Scopaeus gracilis group (Coleoptera, Staphylinidae, Paederinae), with description of new species from Sardinia, southern Africa and Middle East. - Mitteilungen aus dem Museum für Naturkunde in Berlin, Deutsche Entomologische Zeitschrift (N.F.) 54 (2): 195-218. https://doi.org/10.1002/mmnd.200700019.

FrIsCH, J. 2009: A revision of the Scopaeus mutatus species group (Staphylinidae, Paederinae), with description of new species from Anatolia and the Caucasus. Deutsche Entomologische Zeitschrift 56 (2): 271-287. - https://doi.org/10.1002/mmnd.200900024.

Frisch, J. 2010: On the taxonomy and biogeography of West Palaearctic Scopaeina Mulsant \& ReY (Staphylinidae, Paederinae). - Deutsche Entomologische Zeitschrift 57 (2): 159-202. - https://doi. org/10.1002/mmnd.201000016.

Frisch, J. 2012: A revision of the West Palaearctic Scopaeus sericans species group (Staphylinidae, Paederinae: Scopaeina), with description of two new species from Central Asia. - Deutsche Entomologische Zeitschrift 59 (2): 277-295. - https://doi.org/10.1002/ mmnd.201200023.

Ganglbauer, L. 1895: Die Käfer von Mitteleuropa. 2. Band: Staphylinidae und Pselaphidae. - Verlag von Carl Gerold's Sohn, Wien: 850 pp.

Gildenkov, M. Y. 2000: Obzor Palearkticheskikh vidov roda Thinodromus (Coleoptera, Staphylinidae). Soobshchenie 2. - Zoologichesky Zhurnal 79: 918-927.

Gildenkov, M. Y. 2001: Fauna Carpelimus Palearktiki (Coleoptera: Staphylinidae). Problemy vida i vidoobrazovaniia. Chast Pervaya. Istoria izucheniya. Morfo-ekologicheskie osobennosti. Sistema roda. Opisaniia vidov. - SGPU, Smolensk: 304 pp.
GILDENKov, M. Y. 2009: Novye dannye o rasprostanenii $\mathrm{v}$ palearktike vidov roda Thinodromus i vidov roda Carpelimus iz podrodov Carpelimus s. str., Paratrogophloeus, Bucephalinus (Coleoptera, Staphylinidae). - Izvestia Smolenskogo Gosudarstvennogo Universiteta 6 (2): 25-42.

Gildenkov, M. Y. 2015: Fauna Carpelimus starogo sveta (Coleoptera: Staphylinidae). - Izdatelstvo SmolGU, Smolensk: 1-413.

Hammond, P.; Morgan, A. \& Morgan, A. V. 1979: On the gibbulus group of Anotylus, and fossil occurrences of Anotylus gibbulus (Staphylinidae). - Systematic Entomology 4: 215-221. - https://doi. org/10.1111/j.1365-3113.1979.tb00637.x.

Herman, L. H. 1986: Revision of Bledius. Part IV. Classification of species groups, phylogeny, natural history, and catalogue (Coleoptera, Staphylinidae, Oxytelinae). - Bulletin of the American Museum of Natural History 184: 1-368. - http://hdl.handle. net $/ 2246 / 580$.

Herman, L. H. 2001: Catalog of the Staphylinidae (Insecta: Coleoptera). 1758 to the end of the second millennium. Volumes I-VII. - Bulletin of the American Museum of Natural History 265: 4218 pp.

Нетsснко, A. 1922: Hans Leder. - Wiener Entomologische Zeitung 39 (1-4): 95-96.

Hlaváč, P. \& Stevanovíć, M. 2013: A review of the subgenus Cladoconnus Reitter of the genus Euconnus Thomson (Coleoptera: Staphylinidae: Scydmaeninae) from the Balkan Peninsula, Turkey and Caucasus. - Zootaxa 3646 (4): 401-425. - http:// dx.doi.org/10.11646/zootaxa.3646.4.5.

Носннuтн, J. H. 1847: Enumeration der Rüsselkäfer, welche vom Baron Maximilian von Chaudoir und vom Baron A. v. Gotsch auf ihren Reisen im Kaukasus und in Transkaukasien im Jahre 1845 gesammelt wurden; nebst Beschreibung der neuentdeckten Arten. Bulletin de la Société Impériale des Naturalistes de Moscou 20: 448-587.

Hochнuth, J. H. 1849: Die Staphylinen-Fauna des Kaukasus und Transkaukasiens. - Bulletin de la Société Impériale des Naturalistes de Moscou 22 (1): $18-214$.

Hochнuтн, J. H. 1851: Beitraege zur naeheren Kenntniss der Staphylinen Russlands. Enthaltend Beschreibung neuer Genera und Arten, nebst Erläuterungen noch nicht hinlänglich bekannter Staphylinen des russischen Reichs. - Bulletin de la Société Impériale des Naturalistes de Moscou 24 (2): 3-58.

Носннuтн, J. H. 1862: Beiträge zur näheren Kenntnis der Staphyliniden Russlands. - Bulletin de la Société Impériale des Naturalistes de Moscou 35 (3): 1-113.

Horion, A. 1963: Faunistik der mitteleuropäischen Käfer. Staphylinidae. 1. Teil. Micropeplinae bis Euaesthetinae. - Aug. Feyel, Überlingen-Bodensee: $412 \mathrm{pp}$. 
Horion, A. 1965: Faunistik der mitteleuropäischen Käfer. Band X: Staphylinidae. 2. Teil. Paederinae bis Staphylininae. - Ph. C. W. Schmidt, ÜberlingenBodensee: 335 pp.

Horion, A. 1967: Faunistik der mitteleuropäischen Käfer. Bd. XI: Staphylinidae, 3. Teil: Habrocerinae bis Aleocharinae (ohne Subtribus Athetae). Ph. C. W. Schmidt, Überlingen-Bodensee: 419 pp.

HromádKa, L. 1990: Eine neue Stenus-Art aus Armenien (Coleoptera, Staphylinidae). - Entomofauna 11 (17): 281-284. - https://www.zobodat.at/pdf/ENT_0011_ 0281-0284.pdf.

IABLOKOFF-KHNZORIAN, S. M. 1956: Chetyre novykh vida zhestkokrylykh iz Armyanskoy SSR (Coleoptera: Insecta). - Doklady Akademii Nauk Armyanskoy SSR 22: 135-139.

IABLOKOFF-KHNZORIAN, S. M. 1957a: Zhestkokrylye duba v Armyanskoi SSR. - Zoologichesky Sbornik Akademii Nauk Armyanskoy SSR 10: 59-152.

IABLOKOFF-Khnzorian, S. M. 1957b: Dva novykh zhuka-stafilina na Armyanskoi SSR (Coleoptera, Staphylinidae). - Zoologicheskiy Zhurnal 36: 291-293.

IABLOKOFF-Khnzorian, S. M. 1957c: Novye vidy zhestkokrylykh iz na Armyanskoy SSR i Nakh. ASSR. - Zoologichesky Sbornik Akademii Nauk Armyanskoy SSR 10: 153-183.

IABLOKOFF-KHnzorian, S. M. 1959: Novye vidy zhestkokrylykh (Coleoptera, Insecta) iz Armyanskoy SSR i nakhichevanskoy ASSR. II. - Zoologichesky Sbornik Akademii Nauk Armyanskoy SSR 11: 65-68.

IABLOKOFF-Khnzorian, S. M. 1960: Chetyre novykh zhestkokrylykh iz Armyanskoy SSR (Insecta, Coleoptera). - Zoologichesky Zhurnal 39: 1881-1884.

IABlokoff-Khnzorian, S. M. 1961: Coléoptères nouveaux de l'Arménie Soviétique. - Notulae Entomologicae 40 [1960]: 140-153.

IABloKofF-KHnzorian, S. M. 1962: Novye vidy zhestkokrylykh iz Zakavkazya. New species of Coleoptera from Transcaucasus (Insecta-Coleoptera). - Zoologichesky Sbornik Zoologichesky Institut, Akademii Nauk Armyanskoy SSR 12: 99-124.

IABLOKOFF-Khnzorian, S. M. 1964a: New genera and species of Coleoptera from Transcaucasus and Middle Asia. - Zoologichesky Sbornik Akademii Nauk Armyanskoy SSR 13: 151-186.

IABLOKOFF-Khnzorian, S. M. 1964b: Pholeophil, nidicol and myrmecophilous beetles in Armenian SSR. Zoologichesky Sbornik Akademii Nauk Armyanskoy SSR 13: 187-212.

IABLOKOFF-KHNZORIAN, S. M. 1966: Dva novykh vysokogornykh vida zhestkokrylykh-stafilinov s Aragatsa. - Doklady Akademii Nauk Armyanskoy SSR 42: 174-175.

IABLOKOFF-KHNZORIAN, S. M. 1985: Zhuki-tchelnovidki (Coleoptera, Scaphidiidae) fauny SSSR. - Entomologicheskoe Obozrenie 64 (1): 132-143.
IABLOKOFF-Khnzorian, S. M. 1989: Dva novykh Kavkaszkikh predstavitelia iz roda Geodromicus Redt. (Coleoptera Staphylinidae). - Doklady Akademii Armyanskoy SSR 87 [1988]: 134-139.

Jakobson, G. G. 1906-10: Zhuki Rossii i zapadnoy Evropy. Rukovodstvo $\mathrm{k}$ opredieleniyu zhukov. Sankt-Petersburg: A. F. Devrien, 1024 pp. + lxxxiii pl.

JÁszaY, T. \& Hlaváč, P. 2006: A revision of the Palaearctic species of the genus Dropephylla (Coleoptera: Staphylinidae: Omaliinae). - Entomological Problems 36 (1): 31-62.

Kalashian, M. 1990: Novy yed roda Zibus SAulcy (Coleoptera, Pseaphidae) iz okrestnostei Erevana. - Doklady Akademie Nauk Armyanskoy SSR 90: 185-187.

Karaman, Z. 1940: Revision der Pselaphiden (Col.). 1. Tribus Pselaphini. - Glasnik, Bulletin de la Société Scientifique de Skopje 22: 115-128.

Karaman, Z. 1974: Fortsetzung der Revision der balkanischen Vertreter der Gattung Euconnus Tномs. (Col., Scydmaenidae). - Acta Entomologica Jugoslavica 10 (1-2): 125-145.

Kirshenblat, Y. D. 1932: Obzor zhukov roda Paederus FABR. vstrechaiushchikhsia na territorii SSSR. - Parazitologicheskiy Sbornik Zoologicheskogo Instituta Akademii Nauk SSSR 3: 215-222.

Kirshenblat, Y. D. 1951: Novye Palearkticheskie Staphylinidae (Coleoptera). - Entomologicheskoe Obozrenie 31: 541-545.

Косн, C. 1937: Beitrag zur Systematik und geographischen Verbreitung der Achenium-Arten (Col. Staph.). Pubblicazioni del Museo Entomologico "Pietro Rossi" (Duino) 2: 51-187.

Kocian, M. 1997: A revision of Western Palearctic species of the genus Ischnosoma STEPHENS (Coleoptera, Staphylinidae: Tachyporinae). - Acta Universitatis Carolinae, Biologica 40 [1996]: 241-299.

Kolenati, F. A. R. 1846: Meletemata entomologica. Fascicule III. Brachelytra Caucasi cum distributione geographica adnexis pselaphinis, scydmaenis, notoxidibus et xylophagis. - Typis Imperialis Academiae Scientiarum, Petropoli: 44 pp., pls. XII-XIV.

Kolenati, F. A. R. 1858: Reiseerinnerungen. Erster Theil. Die Bereisung Hocharmeniens und Elisabethopols, der Schekinschen Provinz und des Kasbek im CentralKaukasus. - Rudolf Kuntze, Dresden: I-VI, 1-289.

LAttin, G. DE 1967: Grundriss der Zoogeographie. Gustav Fischer Verlag, Stuttgart: 602 pp.

Leder, H. 1880: Beitrag zur kaukasischen KäferFauna. - Verhandlungen der Kaiserlich-Königlichen Zoologisch-Botanischen Gesellschaft in Wien 29 [1879]: 451-488.

Leder, H. 1881: Beitrag zur kaukasischen KäferFauna. Unter Mitwirkung von Dr. Eppelsheim in Grünstadt und Edmund Reitter in Wien. III. Stück. - Verhandlungen der Kaiserlich-Königlichen Zoologisch-Botanischen Gesellschaft in Wien 30 [1880]: 501-518. 
LıKovskŕ, Z. 1971: Einige west- und mittelasiatische Aleochara-Arten (Coleoptera, Staphylinidae). - Acta Faunistica Entomologica Musei Nationalis Pragae 14 (161): 93-100.

LıKovský, Z. 1981: Ergebnisse der tschechoslovakischiranischen entomologischen Expeditionen nach dem Iran. Coleoptera: Staphylinidae, Subtribus Aleocharae. - Acta Entomologica Musei Nationalis Pragae 40: 359-370.

LöвL, I. 1970: Revision der paläarktischen Arten der Gattungen Scaphisoma LEACH und Caryoscapha Ganglbauer der Tribus Scaphisomini (Col. Scaphidiidae). - Revue Suisse de Zoologie 77 (4): 727-799.

Löbl, I. \& Besuchet, C. 2004: Pselaphinae; pp. 272-329. - In: Löbl, I. \& Smetana, A. (eds), Catalogue of Palaearctic Coleoptera. Volume 2. Hydrophiloidea - Histeroidea - Staphylinoidea. - Apollo Books, Stenstrup: $942 \mathrm{pp}$.

Luze, G. 1900: Revision der europäischen und sibirischen Arten der Staphyliniden-Gattung Tachinus GRAV., nebst zwei Bestimmungstabellen. - Verhandlungen der Kaiserlich-Königlichen Zoologisch-Botanischen Gesellschaft in Wien 50: 475-508.

LuzE, G. 1901: Bolitobiini. Revision der paläarktischen Arten der Staphyliniden-Gattungen Bryocharis Boisd. et LAC., Bolitobius Mannh., Bryoporus KRAATZ und Mycetoporus MANNH. - Verhandlungen der Kaiserlich-Königlichen Zoologisch-Botanischen Gesellschaft in Wien 51: 662-746.

LuzE, G. 1902: Revision der paläarktischen Arten der Staphyliniden-Gattungen Anthophagus GravH. und Hygrogeus ReY. - Verhandlungen der KaiserlichKöniglichen Zoologisch-Botanischen Gesellschaft in Wien 52: 505-530.

Luze, G. 1903: Revision der paläarktischen Arten der Staphylinidengattung Geodromicus ReDTenb. - Verhandlungen der Kaiserlich-Königlichen Zoologisch-Botanischen Gesellschaft in Wien 53: 103-117.

Mannerheim, C. G. von 1830: Précis d'un nouvel arragement de la famille des brachélytres de l'ordre des insectes coléoptères. - Academie Imperiale des Sciences de St. Petersbourg: 87 pp.

Makranczy, Gy. 2014: Revision of the genus Ochthephilus Mulsant \& ReY, 1856 (Coleoptera: Staphylinidae, Oxytelinae). - Revue Suisse de Zoologie 121 (4): 457-694.

Maruyama, M. 2006: Revision of the Palearctic species of the myrmecophilous genus Pella (Coleoptera, Staphylinidae, Aleocharinae). - National Science Museum Monographs 32: 1-207.

Moreno-Sanchez, R. \& Sayadyan, H. Y. 2005: Evolution of the forest cover in Armenia. - International Forestry Review 7 (2): 113-127.

Motschulsky, V. DE 1850: Die Kaefer Russlands. W. Gautier, Moscau: I-IV, 1-141.
Motschulsky, V. DE 1860: Énumération des nouvelles espèces de coléoptères rapportées de ses voyages. $3^{\mathrm{e}}$ partie. - Bulletin de la Société Impériale des Naturalistes de Moscou 33 (2): 539-588.

MülleR, J. [G.] 1923: Contributo alla conoscenze del genere Staphylinus L. - Bollettino della Società Entomologica Italiana 55: 135-144.

Neukirchen, F. 2011: Bewegte Bergwelt. Gebirge und wie sie entstehen. - Spectrum Akademischer Verlag, Heidelberg: $232 \mathrm{pp}$.

PACE, R. 1983: Nuove specie europee ed asiatiche del genere Geostiba Thomson (Coleoptera Staphylinidae). - Giornale Italiano di Entomologia 1: 129-139.

Pace, R. 1989: Monografia del genere Leptusa KraAtz (Coleoptera Staphylinidae). - Memorie del Museo Civico di Storia Naturale di Verona (II ${ }^{\circ}$ Serie), Sezione Scienze della Vita (A: Biologica) 8: 1-307.

PAśniK, G. 2006: A revision of the World species of the genus Tachyusa Erichson, 1837 (Coleoptera, Staphylinidae: Aleocharinae). - Zootaxa 1146: $1-152$.

Petrenko, A. A. 1980: Novye i maloizvestnye dlya fauny Kavkaza zhuki-stafilinidy (Coleoptera, Staphylinidae). - Vestnik Zoologii 1980 (5): 81-83.

Plöchinger, B. 1979: Das transkaukasische Armenien, ein Teil des Alpinen Mediterranen Orogens. Verhandlungen der Geologischen Bundesanstalt 1979 (2): 195-203.

Puthz, V. 1967: Über Stenus (Parastenus) alpicola FAUVEL und andere abweichend gebaute paläarktische Parastenus-Arten (Col., Staphylinidae). - Annales Entomologici Fennici 33: 226-256.

Puthz, V. 1972a: Das subgenus "Hemistenus" (Col., Staphylinidae). - Annales Entomologici Fennici 38: 75-92.

Puthz, V. 1972b: Zur Staphylinidenfauna des Balkans: Die bisher aus Jugoslawien und angrenzenden Ländern bekannten Steninen (Coleoptera, Staphylinidae). - Wissenschaftliche Mitteilungen des Bosnisch-Herzegovinischen Landesmuseums 1 (C Naturwissenschaft) [1971]: 239-292.

Puthz, V. 1979: Wissenschaftliches Ergebnis der zoologischen Expedition des Nationalmuseums in Prag nach der Türkei. Coleoptera-Staphylinidae, Subfam. Steninae. - Acta Entomologica Musei Nationalis Pragae 39 [1977]: 319-327.

Puthz, V. 1981: Neue westpaläarktische Stenus, vorwiegend aus dem Genfer Museum (Coleoptera, Staphylinidae). - Revue Suisse de Zoologie 88 (3): 693-706.

Puthz, V. 1983: Einige Stenus-Arten aus dem Kaukasus und Elburs (Insecta: Coleoptera: Staphylinidae). Senckenbergiana Biologica 63 [1982]: 347-362.

Puthz, V. 2003: Stenus alpicola Fauvel und seine Nahverwandten (Coleoptera, Staphylinidae). Entomologische Blätter 98 (2) [2002]: 89-110. 
Puthz, V. 2008: Stenus Latreille und die segenreiche Himmelstochter (Coleoptera, Staphylinidae). - Linzer Biologische Beiträge 40 (1): 137-230. - https://www. zobodat.at/pdf/LBB_0040_1_0137-0230.pdf.

Puthz, V. 2009: Neue und alte paläarktische StenusArten (Col., Staphylinidae). - Zeitschrift der Arbeitsgemeinschaft Österreichischer Entomologen 61: 29-50.

Puthz, V. 2012: Über einige paläarktische Stenus-Arten (Coleoptera, Staphylinidae). - Entomologische Blätter und Coleoptera 108: 151-158.

Puthz, V. 2016: Übersicht über die Arten der Gattung Dianous Leach group II (Coleoptera, Staphylinidae). Linzer Biologische Beiträge 48 (1): 705-778. - https:// www.zobodat.at/pdf/LBB_0048_1_0705-0778.pdf.

Reitter, E. 1889: Neue Coleopteren aus Europa, den angrenzenden Ländern und Sibirien, mit Bemerkungen über bekannte Arten, Siebenter Theil. - Deutsche Entomologische Zeitschrift 1889: 273-288.

Reitter, E. 1905a: Neun neue Coleopteren aus der palaearktischen Fauna. - Wiener Entomologische Zeitung 24: 201-206. - https://www.zobodat.at/pdf/ WEZ_24_0201-0206.pdf.

Reitter, E. 1905b: Übersicht der Arten der ColeopterenGattung Proteinus LATR. aus Europa und dem Kaukasus. - Wiener Entomologische Zeitung 24: 226-228. - https://www.zobodat.at/pdf/WEZ_24_ 0226-0228.pdf.

Reitter, E. 1908: Bestimmungs-Tabelle der Staphyliniden-Gruppen der Othiini und Xantholinini aus Europa und den angrenzenden Ländern. Verhandlungen des Naturforschenden Vereines in Brünn 46 [1907]: 100-124. - https://www.zobodat.at/ pdf/Verh-naturf-Ver-Bruenn_46_0100-0124.pdf.

Reitter, E. 1909: Fauna Germanica. Die Käfer des Deutschen Reiches. Nach der analytischen Methode bearbeitet. 2. - K. G. Lutz, Stuttgart: 1-392.

Roubal, J. 1911: Koleopterologické výsledky mé cesty na Kavkaz v červenci r. 1910 - Quid novi de Coleopterorum Caucasi ad orientem vergentis fauna in meo itinere Julio mense anni 1910 suscepto cognoverim. - Časopis České Společnosti Entomologické 8: 1-18.

Ryvkin, A. B. 1990: Stafilinidy podsemeistva Steninae (Coleoptera, Staphylinidae) Kavkaza i sopredelnykh territorii. Pp. 137-234. - In: Striganova, B. (ed.), Fauna nazemnykh bespozvonochnykh Kavkaza. Sbornik Nauchnykh Trudov. -Akademiia Nauka SSSR, Moskva: 237 pp.

Sabella, G.; Bückle, C.; Brachat, V. \& Besuchet, C. 2004: Revision der paläarktischen Arten der Gattung Brachygluta Thomson, 1859 (Coleoptera, Staphylinidae) (1. Teil). - Muséum d'Histoire Naturelle, Genève: 283 pp.

Sabella, G. \& Kurbatov, S. A. 2002: Contribution to the knowledge of the genus Tychus LEACH, 1817 (Coleoptera: Staphylinidae: Pselaphidae). -
Annales de la Société Entomologique de France (N. S.) 38 (3): 299-317.

Scheerpeltz, O. 1955: Eine neue Art der Gattung Platystethus MannH., mit einer Bestimmungstabelle der westpaläarktischen Arten und Formen dieser Gattung (Col. Staphylinidae). - Koleopterologische Rundschau 33: 78-88.

Scheerpeltz, O. 1957: Vorläufige Diagnosen einiger neuen paläarktischen Arten und Formen der Gattung Paederidus Muls. Rey, Paederus Fabr. (mit den neuen Untergattungen Eopaederus, Paederus s. str. nov., Heteropaederus, Dioncopaederus und Oedopaederus), Parameropaederus nov. gen., Lobopaederus nov. gen. und Megalopaederus nov. gen. - Memorie del Museo di Storia Naturale della Venezia Tridentina 11 (1) (1956-1957): 447-475.

Scheerpeltz, O. 1958: Wissenschaftliche Ergebnisse der von Herrn Dr. K. Lindberg, Lund, im Jahre 1956, nach der Türkei und Armenien unternommenen Reise. Coleoptera - Staphylinidae. - Entomologisk Tidskrift (Supplementum) 78 [1957]: 3-37.

Scheerpeltz, O. 1966a: Die neue Systematik der Großgattung Leptusa KraAtz (Col. Staphylinidae). - Verhandlungen der Zoologisch-Botanischen Gesellschaft in Wien 105/106: 5-55. - https://www. zobodat.at/pdf/VZBG_105-106_0005-0055.pdf.

Scheerpeltz, O. 1966b: Eine neue Art der Grossgattung Staphylinus L., neue Subgenera und Bemerkungen über einige bereits bekannte paläarktische Arten dieser Grossgattung (Col.). - Nachrichtenblatt der Bayerischen Entomologen 15: 105-117. - https://www. zobodat.at/pdf/NachBlBayEnt_015_0105-0117.pdf.

Schillhammer, H. 2003: On some Central Asian species of the Gabrius astutus group (Insecta: Coleoptera: Staphylinidae). - Annalen des Naturhistorischen Museums in Wien 104 B: 353-361. - https://www. zobodat.at/pdf/ANNA_104B_0353-0361.pdf.

Schillhammer, H. 2004: New records, synonyms and nomenclatoral changes in the tribe Staphylinini (Insecta: Coleoptera: Staphylinidae). - Annalen des Naturhistorischen Museums in Wien 105 B: 319-325. - $\quad$ https://www.zobodat.at/pdf/ANNA_105B_0319 -0325.pdf.

Schneider, O. \& Leder, H. 1878: Beiträge zur Kenntniss der kaukasischen Käferfauna. - Verhandlungen des Naturforschenden Vereines in Brünn 16 [1877]: 3-258, tab. I-IV.

SCHÜLKE, M. 1989: Bemerkungen zur Verbreitung und Synonymie einiger Arten der Gattung Tachinus Gravenhorst (Coleoptera, Staphylinidae: Tachyporinae). - Entomologische Nachrichten und Berichte 33 (5): 230-232.

Schülke, M. 2004: Zur Taxonomie der Unterfamilie Tachyporinae (Coleoptera: Staphylinidae). Typendesignationen, Neukombinationen, Untergattungszuordnungen, Nomina nova und neue Synonymien. - Linzer Biologische Beiträge 36 (2): 919-1000. 
SChÜLKe, M. 2008: Neumeldungen von paläarktischen Tachyporinen und Ergänzungen zum "Catalogue of Palaearctic Coleoptera, Volume 2" (Coleoptera, Staphylinidae, Tachyporinae). - Linzer Biologische Beiträge 40 (1): 927-942.

Schülke, M. 2019a: Zur Identität der von Gaston Fagel beschriebenen Arten der Gattung Mycetoporus Mannerheim, 1830 mit Beschreibung neuer Arten der Mycetoporus baudueri Gruppe (Coleoptera, Staphylinidae, Tachyporinae). - Linzer Biologische Beiträge 51 (1) (in press).

SchüLKe, M. 2019b: Drei neue westpaläarktische Arten der Sepedophilus testaceus Gruppe (Coleoptera, Staphylinidae, Tachyporinae). - Linzer Biologische Beiträge 51 (1) (in press).

Schülke, M. \& KocIan, M. 2000: Revision der Artgruppe des Mycetoporus nigricollis STEPHENs, 1835 (Coleoptera, Staphylinidae, Tachporinae). Entomologische Blätter 96 (2): 81-126.

Schülke, M. \& Smetana, A. 2015: Staphylinidae, pp. 304-1134. - In: LöBL, I. \& LöвL, D. (eds), Catalogue of Palaearctic Coleoptera. New updated Edition. Volume 2. Hydrophiloidea - Staphylinoidea. Revised and updated edition. - Brill, Leiden: xxvi + $1702 \mathrm{pp}$.

Semenov, V. B. 2003: Die kaukasischen und mittelasiatischen Arten der Gattung Pronomaea ERICHSON, 1837 (Coleoptera: Staphylinidae: Aleocharinae). Russian Entomological Journal 12: 199-202.

Smetana, A. 2004: Staphylinidae; pp. 207-272, 329-495, 504-698. - In: Löвl, I. \& SmetanA, A. (eds), Catalogue of Palaearctic Coleoptera. Volume 2. Hydrophiloidea - Histeroidea - Staphylinoidea. Apollo Books, Stenstrup: 942 pp.

Solodovnikov, A. 2002: Taxonomy and faunistics of some species of Quedius Stephens, 1829 from the Caucasus and Asia Minor (Coleoptera: Staphylinidae). - Koleopterologische Rundschau 72: 137-158.

Solodovnikov, A. 2004: Taxonomy and faunistics of some West Palearctic Quedius StePhens subgenus Raphirus Stephens (Coleoptera: Staphylinidae: Staphylininae). - Koleopterologische Rundschau 74: 221-243.

Solodovnikov, A. 2005: New and little known species of Quedius from West Palaearctic [sic]. - Zootaxa 902: $1-13$.
Solodovnikov, A. \& Grebennikov, K. A. 2005: Revision of Physetops Mannerheim, 1830, a monotypic genus with the polymorphic species P.tataricus (PALlas, 1773) (Coleoptera, Staphylnidae, Staphylininae). Mitteilungen des Museums für Naturkunde Berlin, Zoologische Reihe 81 (1): 67-80.

Tagliapietra, A. \& Zanetti, A. 2003: Staphylinidae; pp. 90-105. - In: Cerretti, P.; Tagliapietra, A.; Tisato, M.; Vanin, S.; Mason, F. \& Zapparoli, M. (eds.), Artropodi dell'orizzonte del faggio nell' Appennino settentrionale. - Gianluigi Arcari Editore, Mantova: 256 pp.

Ullrich, W. G. 1975: Monographie der Gattung Tachinus Gravenhorst (Coleoptera: Staphylinidae), mit Bemerkungen zur Phylogenie und Verbreitung der Arten. Dissertation zur Erlangung des Doktorgrades der Mathematisch-Naturwissenschaftlichen Fakultät der Christian-Albrechts-Universität zu Kiel. - Kiel: 365 pp., 61 pls.

Ushakov, I. A. 1986: Novy vid roda Xantholinus (Coleoptera, Staphylinidae) iz Zakavkaz'ia. - Zoologichesky Zhurnal 65: 1090-1092.

Vít, S. \& Besuchet, C. 2004: Cephenniini; pp. 203-206. - In: Löbl, I. \& Smetana, A. (eds), Catalogue of Palaearctic Coleoptera. Volume 2. Hydrophiloidea - Histeroidea - Staphylinoidea. - Apollo Books, Stenstrup: $942 \mathrm{pp}$.

VÍT, S. \& HLAvÁč, P. 1998: Review of Euconnus(Tetramelus) of the reitteri group (Coleoptera: Scydmaenidae). Entomological Problems 29 (2): 139-147.

Zanetti, A. 1987: Fauna d'Italia. Coleoptera Staphylinidae Omaliinae. - Calderini, Bologna: 472 pp.

Zanetti, A. 1993: Contributo alla conoscenza degli Eusphalerum del Caucaso, dell'Anatolia e delle regioni vicine. (Coleoptera, Staphylinidae: Omaliinae). Bollettino del Museo Civico di Storia Naturale di Verona 17 [1990]: 213-263.

Zanetti, A. 2002: Studies on Omalium Gravenhorst, 1802 from Turkey, Cyprus, and the Caucasus region, with notes on some European and Asian species (Coleoptera, Staphylinidae: Omaliinae). - Bollettino del Museo Civico di Storia Naturale di Verona. Botanica Zoologia 26: 45-63. 